\title{
A KNOWLEDGE-BASED FRAMEWORK FOR CONSTRUCTION METHODS SELECTION
}

by

\author{
Ibrahim A. Al-Hammad \\ B.A.Sc., King Fahad University of Petroleum \\ and Minerals, Saudia Arabia, 1981 \\ M.A.Sc., The University of Colorado, U.S.A., 1985
}

A THESIS SUBMITTED IN PARTIAL FULFILLMENT OF

THE REQUIREMENTS FOR THE DEGREE OF

DoctoR OF PHILOSOPHY

in

THE FACULTY OF GRADUATE STUDIES

DEPARTMENT OF CIVIL ENGINNERING

We accept this thesis as conforming

to the required standard

THE UNIVERSITY OF BRITISH COLUMBIA

April 1991

(C) Ibrahim A. Al-Hammad, 1991 
In presenting this thesis in partial fulfilment of the requirements for an advanced degree at the University of British Columbia, I agree that the Library shall make it freely available for reference and study. I further agree that permission for extensive copying of this thesis for scholarly purposes may be granted by the head of my department or by his or her representatives. It is understood that copying or publication of this thesis for financial gain shall not be allowed without my written permission.

Department of Cacil Cengmelny

Vancouver, Canada

Date $4 \mathrm{mil} 30^{\text {th }} / 991$ 


\section{Abstract}

The objectives of this thesis are to investigate, formulate, and structure the problem of methods selection, and apply a Knowledge-Based Expert System (KBES) approach. A complete, conceptual KBES framework for the methods selection problem is proposed and selected aspects of it were implemented using NExpert object.

Defined hierarchically, a conceptual method frame consists of the following attributes: design element, construction strategy, construction resources, and construction process model. The roles of the KBES control strategy are to first specify a method and then rank it versus others. In so doing, the control strategy is applied at two levels: a preliminary feasibility level, and a detailed feasibility level. The former is used to reduce the number of available methods and rank them for processing by the latter. The preliminary feasibility part constitutes declarative knowledge with high level premises.

The detailed feasibility level, develops the attributes of the method. This component contains empirical, analytical, and procedural knowledge that draws on the civil engineering knowledge domains of design, analysis and construction. Because the notion of a frame is a useful way of identifying the attributes of a construction method, a 
conceptual frame is used throughout to demonstrate the build-up of the method attributes through preliminary, then detailed feasibility.

An expert system called CMsA (Construction Methods Selection Assistant) was developed to implement a subset of the proposed solution approach with cut-and-Cover tunnelling as the problem domain. CMSA, as designed, constitutes a methods selection shell that can be applied to other domains. It entails a solution paradigm of suggest, Design, Predict, and Analyze operators.

CMSA incorporates previous experience (shallow knowledge) as well as algorithmic procedures (deep knowledge).

Key elements central to CMSA knowledge base include risk, design technical feasibility, resources compatibility, cost and time performance measures, and regulatory constraints. Allowance is made for modelling project context variables. A range of geotechnical conditions were treated for the example problem domain.

The KBES framework proposed for the methods selection problem shows promise for tackling this ill-structured problem, helping to organize site experience, and contributing to productivity improvement. 


\section{Contents}

Abstract

Contents

Figures

Tables

Screens

Listings

Acknowledgement

Acronyms

\section{Introduction}

1.1 Background

1.2 Research objectives and Methodology

1.3 Problem Domain

1.4 Organization of the Thesis

2. Literature Survey for Methods Selection Problem 9

2.1 Introduction 9

2.2 Construction Methods 9

2.2.1 Definition of Construction Methods 12

2.2.2 Terminology Used in the Thesis 16

2.3 Decision Making Model for Method Selection 20

2.3 .1 Background 20

2.3.2 Simulation Techniques 20

2.3.3 Decision Analysis 22

2.3.4 Decision Support Systems (DSS) 23

2.4 Knowledge-Based Expert Systems 28

2.4 .1 KBES Components 29

2.4.2 Expert Systems for Construction Management 33

2.4.3 KBES for Construction Methods Selection 35

\section{Cut-and-Cover Methods in Soft Ground}

3.1 Introduction

3.2 Tunnelling Background

3.3 Cut-and-Cover Tunnelling Alternatives 48

3.3.1. Background

3.3.2 Traditional Cut-and-Cover Tunnelling 50

3.3.3 Milano Cut-and-Cover Tunnelling

3.3.4 Major Operations Common to Cut-and-Cover

Tunnelling

3.4 GWSS Alternatives

3.4 .1 Common Types of GWSSs 57

3.5 Excavation Operations 67

3.6 Factors Affecting Methods Selection and Design 70

3.7 Cut and Cover Tunnelling Project Example 73

$\begin{array}{lll}3.7 .1 & \text { Background } & 73\end{array}$

3.7.2 Lagging and Excavation Construction Cycle 77 


\section{Contents}

4. A KBES Framework for Methods Selection and Design 81

4.1 Introduction $\quad 81$

4.2 A KBES framework for Method Selection 84

4.2.1 General 84

4.2.2 Methods Selection Defined 84

4.2.3 Methods Shell 89

4.2.4 Sketch of System Features and Operation 92

4.3 CMSA Development 106

4.3 .1 Overview 106

4.3.2 Context Modelling 110

4.3.3 Preliminary Feasibility 115

4.3.4 Detailed Feasibility Level 130

4.4 CMSA Risk Component Development and Evaluation 149

5. CMSA Implementation

154

5.1 Introduction

154

5.2 NExpert Object Overview 156

5.2.1 Major NExpert Object Modules 156

5.2.2 NExpert Primitives 160

5.2.3 Viewing Knowledge Structure 167

5.2.4 The Inference Process 168

5.3 CMSA Implementation 174

5.3.1. CMSA Overview 174

5.3.2 Solution Paradigm and Knowledge Base 176

5.3.3 Knowledge Representation 187

5.3.4 Technical Feasibility Part 207

5.3.5 CMSA Chaining and Reasoning (Control
Strategy)

6. The Prototype Example $\quad 219$

6.1 Introduction $\quad 219$

6.2 Example Problem Description $\quad 219$

6.2.1 Session Start 220

6.2.2 Problem Context Specification 224

6.2.3 Modified Example 238

6.3 Risk Component Assessment Implemented 239

6.3.1 Introduction 239

$\begin{array}{ll}6.3 .2 & \text { NExpert Risk Implementation } \\ 6.3 .3 & \text { Risk Routine }\end{array}$

7. Conclusions and Recommendations for Further 250

7.1 Summary 250

7.2 Contribution of The Thesis 251

7.3 Further Research 252

Bibliography 255 


\section{Contents}

Appendix A: Pressures and Moments Computation 262

A. 1 Introduction

A.2 Lateral Pressure Calculations

A.3 Design Principles for Structural Members

Appendix B: Pile Driving Production Rate Derivation

B.1 Introduction

B. 2 Soil/Pile Friction Calculations

B.3 Pile Driving Production Rate Estimation

Appendix C: Interviews $\quad 297$

C. 1 Introduction

C. 2 Minutes of Meeting with Dillingham Contractors

c. 3 Minutes of Meeting with Quadra Construction

C. 4 Project Site Visit

Appendix D: CMSA Partial Listing and Miscellany

D. 1 Introduction

D. 2 Partial Listing of CMSA Knowledge Base

D. 3 Vibratory Hammer Selection Knowledge

D.4 Unit Cost Quotations

D.5 Sample Data Base Files 


\section{Figures}

Figure 2.1

Figure 2.2

Figure 2.3

Figure 2.4

Figure 2.5

Figure 2.6

Figure 2.7

Figure 2.8

Figure 2.9

Figure 2.10

Figure 3.1

Figure 3.2

Figure 3.3

Figure 4.1

Figure 4.2

Figure 4.3

Figure 4.4

Figure 4.5

Figure 4.6

Figure 4.7

Figure 4.8

Figure 4.9

Figure 4.10

Figure 4.11

Figure 4.12

Figure 5.1

Figure 5.2

Figure 5.3

Figure 5.4

Figure 5.5

Figure 5.6

Figure 5.7

Figure 5.8

Figure 5.9

Figure 5.10

Figure 5.11

Figure 5.12

Figure 5.13
Design and Construction Interaction

11

Construction Model Process

Overview of classification system for

Construction Technology

13

Example of Element, Attribute,

Suggested Data structure for selected

Technology

14

Sample Element Activity Frame

28

Example of Knowledge Source

38

40

Labor Component Frame

42

Equipment Component Frame 43

Process Component Frame

43

Cost Comparison for Tunnelling Alternative versus Cut-and-Cover Alternative

Cut and Cover Tunnel Project Isometric

Barchart and Time-space Diagram for the

Seattle Project

48

75

76

Hierarchy of Construction Method Frame

Attributes

85

Construction Methods selection Shell 90

Construction Methods Selection system

Process

94

Detailed Feasibility (Phase 2)

95

Steel Sheet Pile (SSP) Method Frame 107

Prototype Model

Soil Profile Scenarios

109

112

GWSS Frame Synthesis

117

CMSA Rule Execution Loop

119

Drive.c Routine Interface with CMSA

145

States of Nature for Methods Selection 150

Risk Assessment Tree Diagram

153

NExpert Object open AI Environment

Framework

159

NExpert Rule Construct

162

The class and object Hierarchy

Rules Perpendicular to Frames

166

169

Backward Chaining for Inference 171

NExpert Inference Framework

173

Knowledge Base Organization and

Control strategy

177

Implementation Solution Paradigm 178

Design Element Class Hierarchy 190

Design Element Instance Frame 191

Steel Sheet Pile Class in NExpert 192

Steel Sheet Pile Selection Rule 193

Steel Sheet Piles Database (SSP.NXP) 195 


\section{Figures}

Figure 5.14

Figure 5.15

Figure 5.16

Figure 5.17

Figure 5.18

Figure 5.19

Figure 5.20

Figure 5.21

Figure 5.22

Figure 5.23

Figure 5.24

Figure 5.25

Figure 5.26

Figure 6.1

Figure 6.2

Figure 6.3

Figure A.1

Figure A.2

Figure B.1

Figure B.2

Figure B. 3

Figure B. 4
Construction Resource Class Hierarchy

196

Impact Hammer Element

Vibratory Pile Driver Element

Double Acting Hammer Database (DAAH.NXP)

Vibratory Hammer Database (VIBRO.NXP)

Impact Hammer class in NExpert

Hammer selection Rule in NExpert

Construction strategy Class Hierarchy

Construction Process Model Class

Hierarchy

Technical Feasibility Rule

Method Technical Feasibility is True

Technical Feasibility Diagnostic Rule

CMSA Model of Chaining and Reasoning

Instantiation Tree

Risk Assessment Decision Tree for

Steel sheet Pile

Risk Framework Assessment Flow Chart

Pressure and Moments Envelopes

Soil Profile for Two Soils Scenario

Hammer Blow Count versus Soil Resistance

Hammer Blow Count versus Driving Depth

Drive.c Routine Flow Chart

Drive.c Routine Development Flow Chart
197

198

199

199

200

201

205

206

208

212

213

217

237

242

248

264

267

281

285

287

288

\section{Tables}

Table 3.1
Table 3.2
Table 4.1
Table 4.2
Table 4.3
Table 4.4
Table 4.6
Table 5.1
Table 5.2
Table 6.1
Table 6.2
Table A.1
Table A.2
Table B.1
Table B.2
Table B.3
Table C.1

Partial Space of Design/Construction

Elements for Cut-and-Cover Tunnel

Seattle Project General Information

Methods Selection space for GWSS

GWSS Project Context Data

Soil Profile Input-Format 1

Soil Profile Input-Format 2

Hammers for Different Soils

Truth Matrix for NExpert

Knowledge Base statistics

Risk Assessment Data Input summary

"SSP Risk.nxp" File for SSP Alternative

Soil- Types Properties Employed in CMSA

Lagging Members

Values for Angle of Internal Friction

Ultimate skin Friction for Sands

"Out.out" Sample output

Sample Pile Driving Resources Unit Cost 307 


\section{Tables}

Table D. 1 Table D. 2 Table D. 3

\section{Screens}

Screen 6.1 Screen 6.2 Screen 6.3 Screen 6.4 Screen 6.5 Screen 6.6 Screen 6.7 Screen 6.8 Screen 6.9 Screen 6.10 Screen 6.11 Screen 6.12

Screen 6.13 Screen 6.14 Screen 6.15
Vibratory Pile Drivers Sizing

\section{Listings}

Listing B.1 Listing C.1 Listing C. 1 Listing D. 1 Listing D.2 Listing D. 3 Listing D. 4 Listing D. 5 Listing D. 6 Listing D. 7
Knowcess Hypothesis Command Menu CMSA Overview Rule Network Window

CMSA Rule Network window

GWSS Feasible Alternatives

225

Soil Profile specification

Soil Profile specification

225

Soil Profile specification

225

Soil profile specification

226

Water Table Level Input

226

226

Hypothesis "Select_suitable_Sheet_Pile" 228

Hypothesis "Select_Suitable_Hammer" 230

selected_Hammers class and $\bar{i} t s$

Dynamic ōbjects

230

Hammer Efficiency Input

"Drive.txt" Explanatory File

"Results1.nxp" File

Drive.c Routine for Pile Driving 289

Extracted Rules of Thumb 304

Extracted Rules of Thumb (continued) 305

Partial Listing of CMSA 311

Steel Sheet Data Base "SSP. nxp" 336

Soldier Piles Data Base "HP_Pile.nxp" 337

Struts Data Base "strut. nxp" 338

Lagging Data Base "Lag.nxp" 339

Impact Hammer Data Base "Hammer.nxp" 340 Vibratory Hammers Sample Data Base 341 


\section{Acknowledgements}

I am greatly indebted in large part of this thesis to my advisor Professor Alan Russell for his invaluable and incisive input and constructive criticism. Professor Russell provided priceless guidance throughout this research. My gratitude extends to my thesis supervisory committee members of Dr. Caselton, Dr. Sassani, and Dr. S.o. Russell.

Special recognition extends to the Saudi Arabian Educational Mission and British Columbia Science Council for purchasing NExpert object program and IBM 386 to conduct the research work. Special thanks to stuart Brown of Dillingham Co. and John Simonett of Quadra Inc. for granting me interviews and exposing their experience.

Reflecting back at my residence in campus, I have benefited greatly from a number of people. I would like to thank Ronald Yaworsky and Leon Phem for their assistance. Along with other friends, I extend my appreciation to saad Al-Mubiyedh, Mohammad Al-Robesh, Abdul-Aziz Al-Jallal, Tariq Al-Faris, Bernardo de Castello, Rachid Nakeeb, and Malik Ranasinghe -- for their support, encouragement, and friendship that I value.

Everything I have achieved can be attributed to the love and caring that my mother and family have provided. 


\section{Acronyms}

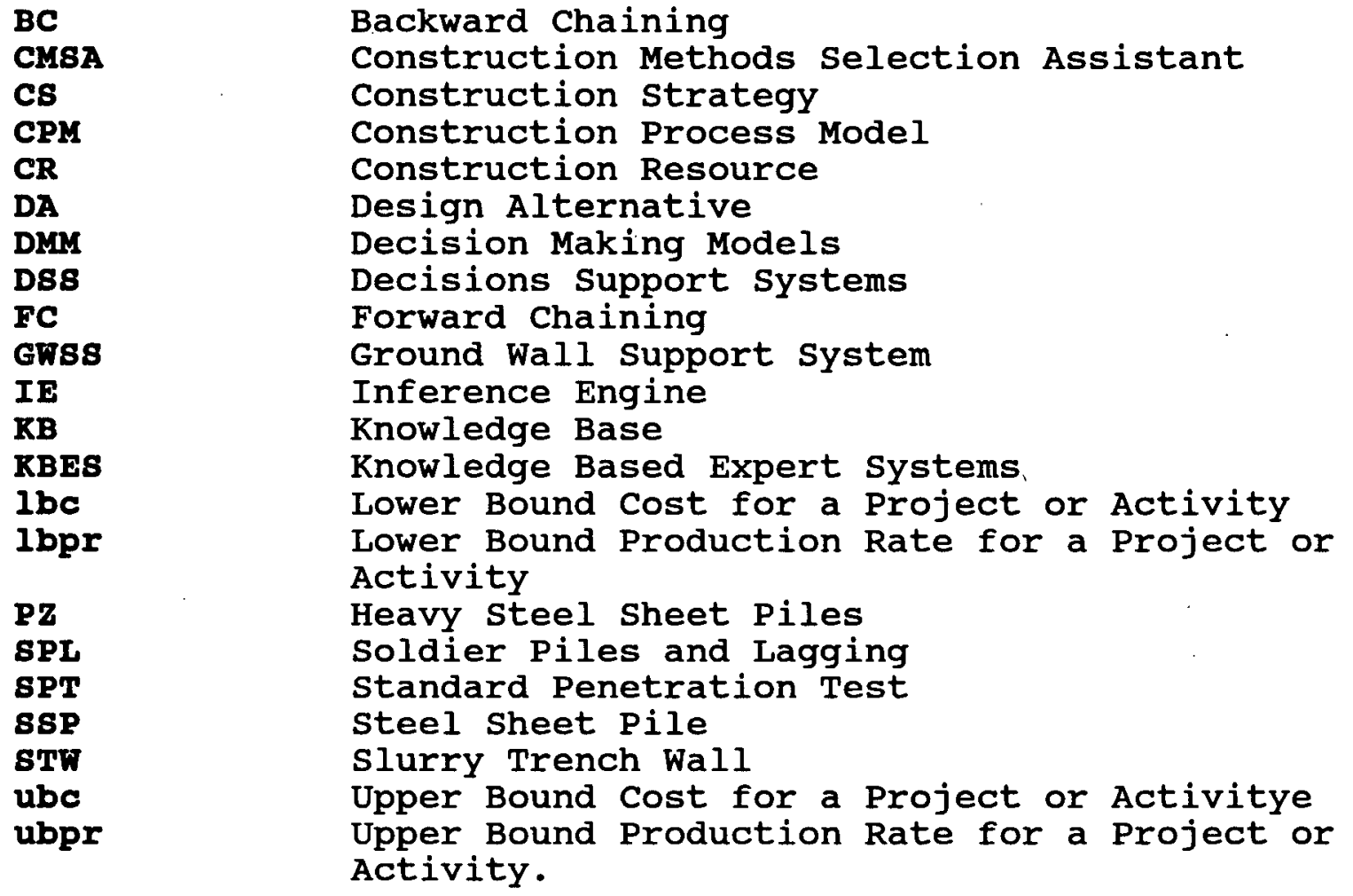




\section{Introduction}

\subsection{Background}

Construction methods selection is a challenging problem. In general, there are numerous alternative methods for performing each major activity in a project. The number of methods available for each activity, and the potential for interaction among them, makes methods selection a complex process. In practice, decision makers rely on past experience from similar projects to provide solutions to the current ones.

Given the selection of a method, traditional techniques, such as network analysis, simulation or decision analysis, can be used to predict time and cost performance. However, these techniques are evaluative, not generative, and do not incorporate heuristic knowledge explicitly. The strength of quantitative modelling techniques lies in their lower level prediction and optimization.

A combination of both descriptive and procedural knowledge is essential to effective methods selection. Consequently, a computerized decision-making tool which embodies a Knowledge Based Expert System (KBES) is worthy of investigation. Such a tool should integrate quantitative and qualitative assessments in order to produce and analyze acceptable solutions. 
The focus of this thesis is on developing a conceptual framework for describing the methods selection problem and on identifying the roles that can be played by knowledge based systems.

\subsection{Research Objectives and Methodology}

An extensive review of the literature revealed that no general statement and formulation of the methods selection problem has been developed. In fact, few researchers ever address the issue, preferring to focus on specific problems. In treating specific problems, research has been directed at applying operations research and systems analysis tools. Little attempt has been made to treat the problem as a design simile as opposed to an analysis one and incorporate construction knowledge in the cognitive process. Complicating the problem is the dimensionality of a construction method in terms of a large number of quantitative and qualitative attributes, the combinatorial problem of combining methods and the multiple criteria used for evaluating a method.

The main goals of this thesis are twofold: to develop a generalized statement and structure for the methods selection problem, and to demonstrate the applicability of a knowledge based approach to this problem. For the latter, a prototype expert system called CMSA (Construction Methods Selection Assistant) has been developed. 
This thesis addresses the methods selection problem in broad terms of organizing, structuring and formulating it, and to propose and implement a KBES framework approach. An appropriate domain example of cut-and-Cover tunnelling will be used. A central research objective is to contribute toward the representation of a construction methods design environment that can handle a wide variety of methods/strategy selection problems. The main function of the environment is to provide the construction user with a subset of feasible methods, given a project context, including preliminary values for design parameters of the short listed methods.

Specific research objectives are as follows:

1. Develop a generalized description and structure for the construction methods selection problem;

2. Identify specific roles that a knowledge based system can fulfill;

3. Develop a detailed representation structure for describing individual methods;

4. Formulate a process of traversing alternate methods and pruning alternatives;

5. Treat multiple decision criteria, including time, cost, and risk;

6. Consider both heuristic and procedural knowledge; and

7. Develop a prototype system, using the context of Cut-and-Cover tunneling, to demonstrate and partially validate findings from objectives 1 through 6 . 
The research methodology employed consists of a number of parts. First, an extensive review of literature was conducted to identify the state-of-the-art and to identify useful approaches to each of the objectives. Second, a specific project context was selected (Cut-and-Cover tunnelling) to bring specificity to the process and to provide a base which could be further generalized by examining other methods selection problems.

Specific research methodologies are as follows:

1. Extensive literature review of previous approaches to the problem as well as conducting field interviews;

2. Specific project context selection (sheet piling GWSS alternative for the cut-and-Cover tunnelling);

3. Devising a wholistic definition for the construction method attributes which serves as a basis for structuring the problem;

4. Employing KBES Techniques, including knowledge acquisition, knowledge representation schemes, and control strategy design; and

5. Utilizing an Expert Systems shell (NExpert object) as a basis for a working prototype to demonstrate viability of suggested approach.

\subsection{Problem Domain}

Cut-and-Cover tunnelling has been chosen as a vehicle to explore and structure the methods selection problem. Its richness is in terms of the number of design and construction alternatives available, the high interaction among construction activities, and its distinctive, 
discrete, repetitive construction activities. Further, the experiential knowledge associated with it spans the geotechnical, structural and construction fields, which makes it a good candidate for exploring a general problem solving framework. The poorly structured nature of the problem domain makes it amendable to a KBES approach.

Problem domain solving, within the thesis context, requires knowledge about soil-structure interaction to design the ground wall support system (GWSS); Cut-and-Cover construction techniques; sequencing and scheduling; optimizing resources; and general project management.

\subsection{Organization of the Thesis}

The remainder of this thesis is organized as follows. chapter 2 examines previous work on construction methods selection. A start is made toward setting out a general definition of the methods selection problem. Conventional approaches. (decision analysis, operations research, simulation and so forth), and knowledge based approaches applied to methods selection problem modelling, are reviewed. The goal of this chapter is to provide a comprehensive view of the state-of-art of previous attempts at modelling the construction methods selection problem.

Chapter 3 examines cut-and-Cover tunnelling, the selected problem domain, and related construction methods, with emphasis placed on the ground wall support system (GWSS) 
design and installation alternatives. Analytical and heuristic design and construction procedures are discussed. Cut-and-Cover tunnelling is a knowledge-rich problem domain that is used as a vehicle to explore the methods selection problem through hypothetical examples. These examples are used to expose how excavation operations are interwoven with GWSS retaining system design attributes.

chapter 4 sets out a framework for structuring the methods selection problem. Design and construction tasks are analyzed for methods selection; a comprehensive method frame definition is introduced; and a conceptual comprehensive KBES approach for method selection is prescribed including system features and control strategy description. The control strategy ranks and synthesizes method alternatives by operating at two levels: a high level, or preliminary feasibility that constitutes declarative knowledge; and detailed feasibility that constitutes procedural knowledge. Evaluation criteria based on costs are used to rank feasible alternatives at both levels. Key elements that make a method accepted or rejected at the two levels are decomposed into procedural and declarative knowledge categories of design, risk, compatibility, performance measures, and regulatory. A simplified approach to risk assessment is presented. 
Treatment of project context variables (state variables), particularly of soil conditions, is also discussed.

Implementation of the model features described in the last part of chapter 4 in the form of an expert system, is described in chapter 5. The shell used is NExpert object. The main features of this shell are briefly highlighted. Knowledge structure and control strategy for CMSA prototype development follow the conceptual model. Frames are used to represent method attributes of design element, construction strategy, construction related resources and construction process models. Rules are used for the control strategy, and to screen alternatives and represent soil profiles. Samples of knowledge constructs utilized in CMSA are provided.

Chapter 6 presents a detailed example of the CMSA prototype synthesizes a Cut-and-Cover tunnelling alternative of steel sheet piles. Conclusions and recommendations for further research are contained in chapter 7 .

A number of Appendices contain details of the algorithms and CMSA knowledge base. Appendix A contains formulas for pressure and moment calculations for the design of the ground wall support system (GWSS). Appendix B covers the mathematical derivations for soil resistance for impact pile driving hammers. They serve as an algorithm to predict the duration of pile driving for a single pile. This 
information is used in turn by the CMSA to predict a total production rate. Appendix $\mathbf{C}$ provides insights into the knowledge acquisition process followed. Covered are the formal and informal interviews conducted and site visits that contributed to the knowledge acquisition process. Appendix D supplies a listing of the CMSA knowledge base and the data bases used. 


\section{Literature Survey for Methods Selection Problem}

\subsection{Introduction}

The literature review in this chapter is divided into three parts. First, previous instances of methods selection problem attributes and definitions are summarized. Subsequently, the attributes and definition for a formal general methods selection problem are set out for this thesis. Second, operations research and systems analysis approaches to specific methods selection problems are examined. Third, recent work involving the application of expert systems to this problem is explored. For the latter approach, it is assumed that the reader is familiar with KBES concepts and terminology.

\subsection{Construction Methods}

To restrict the scope of the thesis problem, we assume that the design of the permanent facilities is fixed. clearly, the kind of methods selection tool investigated in this thesis could be used by designers to better link the design and construction processes, as well as by contractors who seek to optimize their decisions given a design.

Gray (1986) has argued the case for the former where he asserts that the concept of buildability suggests it is advisable to involve the contractor in the early stages of 
the design process. In essence, it means that a deeper understanding of the methods used by contractors to analyze the problems and risks inherent in a design has to be achieved.

Few construction contracts allow early contractor involvement in the planning and design stages. Traditionally, most contracts inhibit this in order to facilitate more competition among bidders. Occasionally, project contract documents may specify the construction methods to be used with provisions that permit the contractor to submit his methods for approval. More frequently, methods are seen as the purview of the contractor and contract documents are silent on the methods to be employed.

In a conventional system, the contractor makes preliminary decisions regarding construction methods based on the information available during bid preparation. If the contractor is successful in being awarded the job, previous decisions are reviewed and further decisions are taken in light of more complete information.

In an idealized system, the contractor is allowed to bring his construction methods knowledge to the early design stage. Figure 2.1 contrasts conventional versus idealized systems (Gray 1985). This figure illustrates interaction among relevant decision categories. For the conventional 
system, the arrows show a unidirectional influence of previous decisions on more recent ones with little modification. For the idealized case, the arrows are bidirectional between the three decision categories.

\section{Approach}

Partles

\section{Conventional}

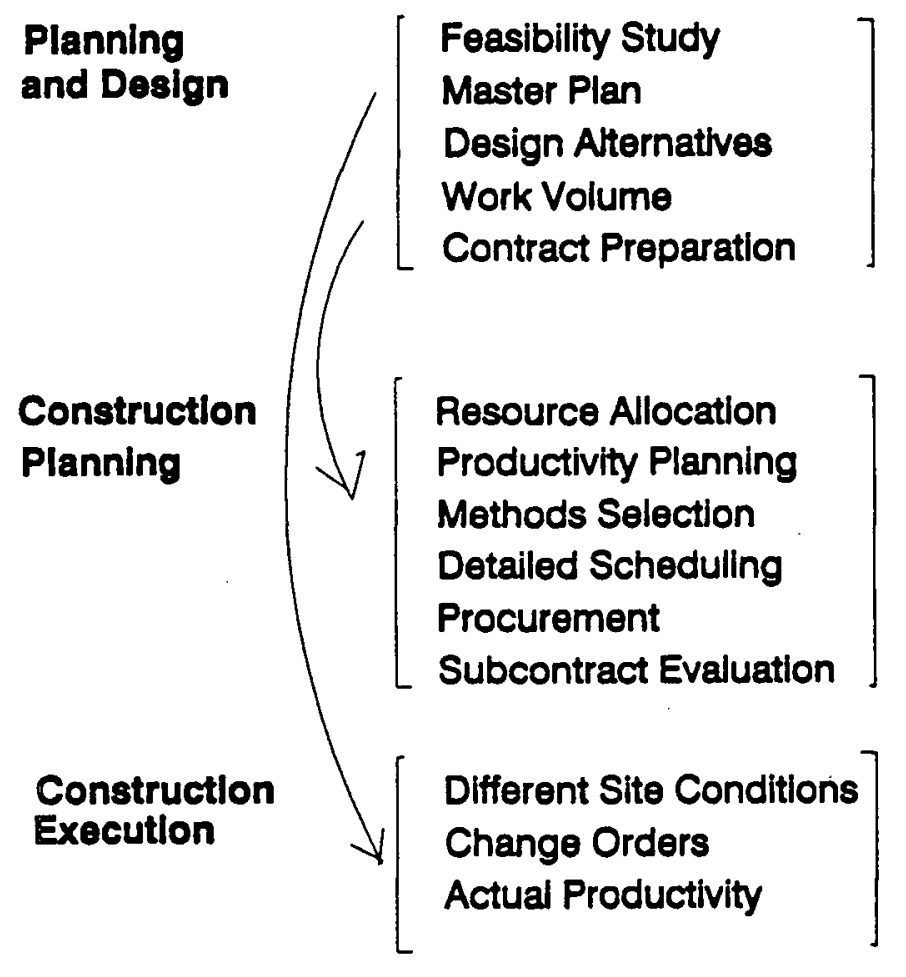

\section{Idesllzed}

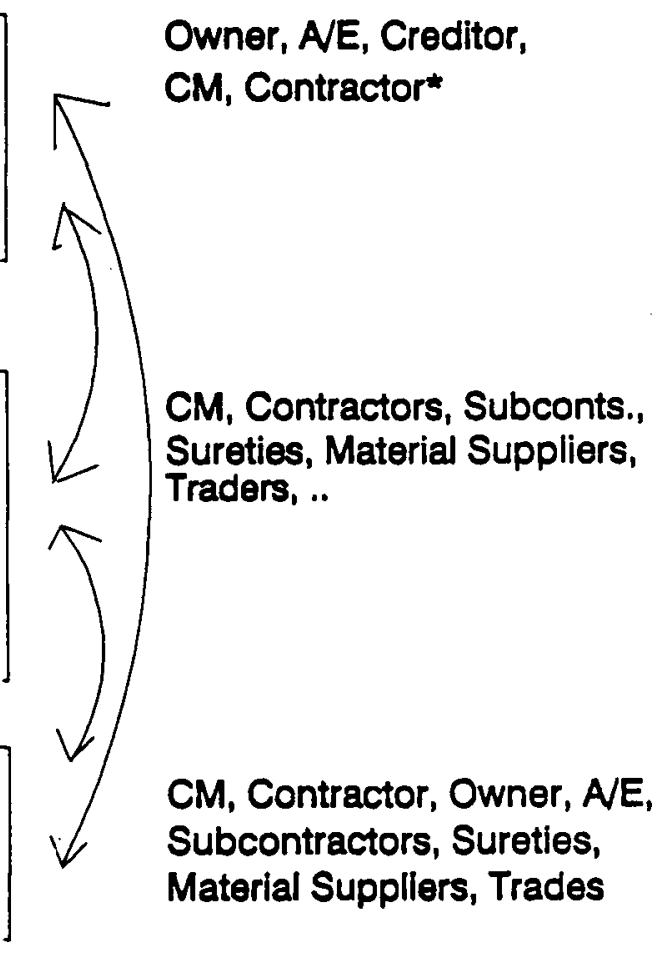

* involved in idealizéd system

Figure 2.1 Design and Construction Interaction [Adapted from Gray 1985] 
The decision making process of methods selection tends to be evolutionary in nature, in which each decision is limited by decisions made at earlier stages, as in a traditional approach, or dynamically, as in an idealistic approach.

\subsubsection{Definition of Construction Methods (Previous work)}

No universal definition of the construction methods selection problem has emerged. Most definitions given in the literature are context sensitive. In this section, the terminology commonly found in the literature is reviewed. We incorporate it in our general definitions on the methods selection problem as appropriate.

Construction technology can be defined as the science of construction involving the judicious use of available materials, methods, and equipment including the necessary planning, preparation, and execution (Merritt 1976).

Tatum described a construction technology classification system that includes a hierarchy of four parts: components, elements, attributes, and values (Tatum 1987 and 1988). Figure 2.2 presents a sketch of a proposed model for the hierarchical construction process as defined by Tatum (1987). Further, figure 2.3 shows the four major components: material and equipment resources, construction applied resources, project requirements and constraints, and construction processes (Tatum 1988). A synthesis of the 
first three components, construction process, represents a model by which performance measures under different scenarios are derived.

The attributes of a construction method can be further elaborated upon as shown in figure 2.4 .

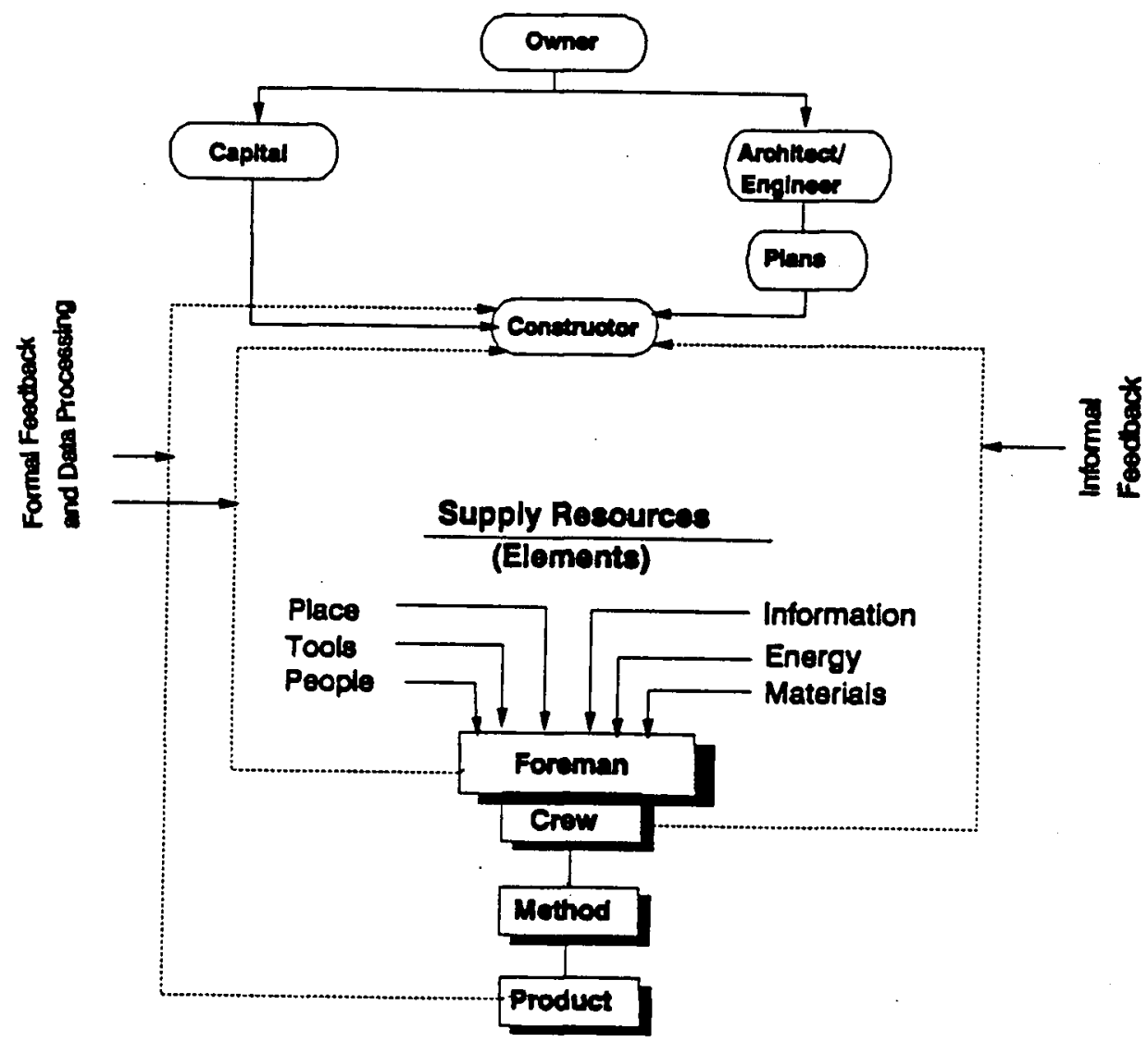

Figure 2.2 Construction Model Process [from Tatum 1987] 


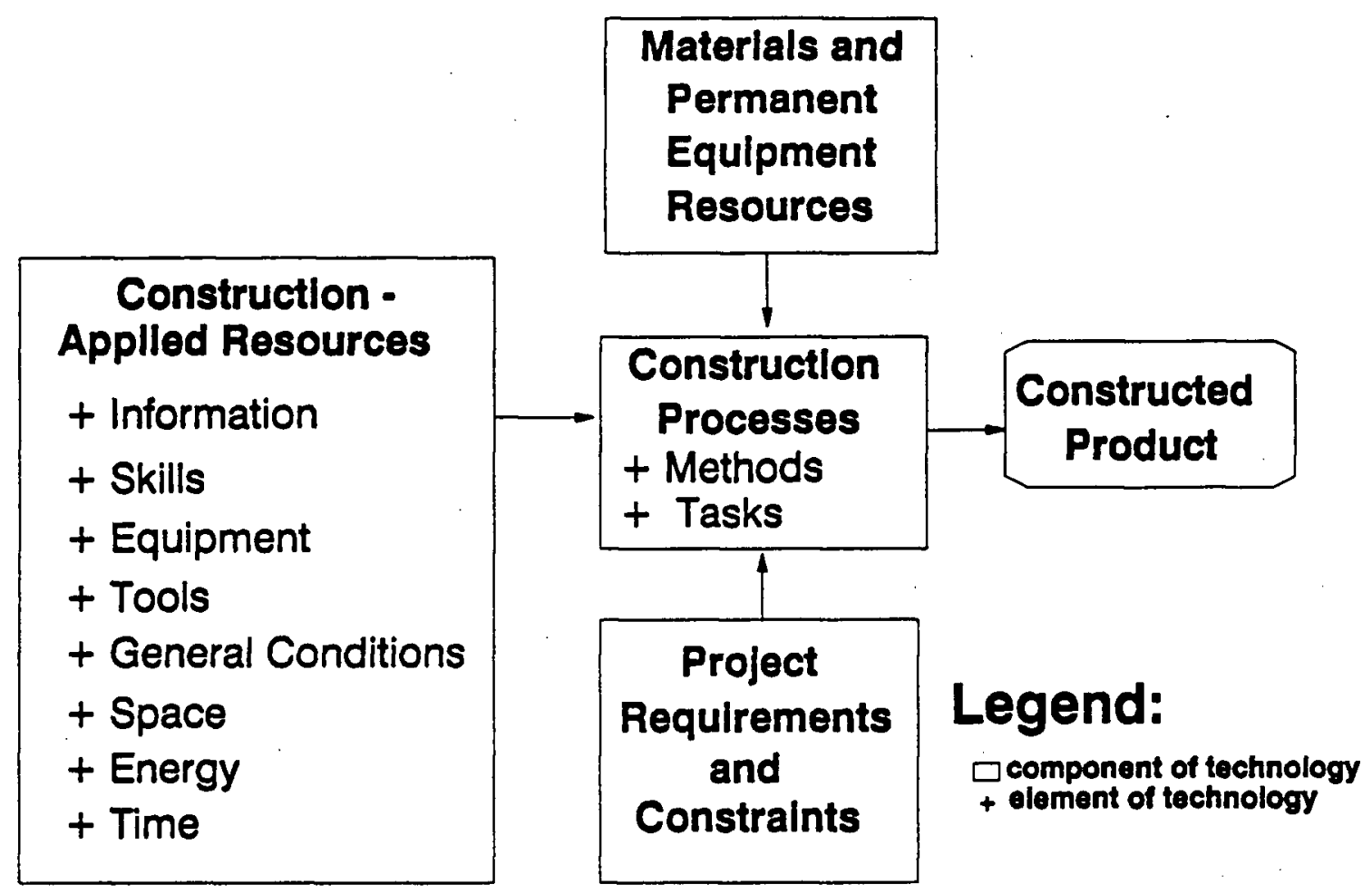

Figure 2.3 Overview of Classification 8ystem for construction Technology [from Tatum 1988]

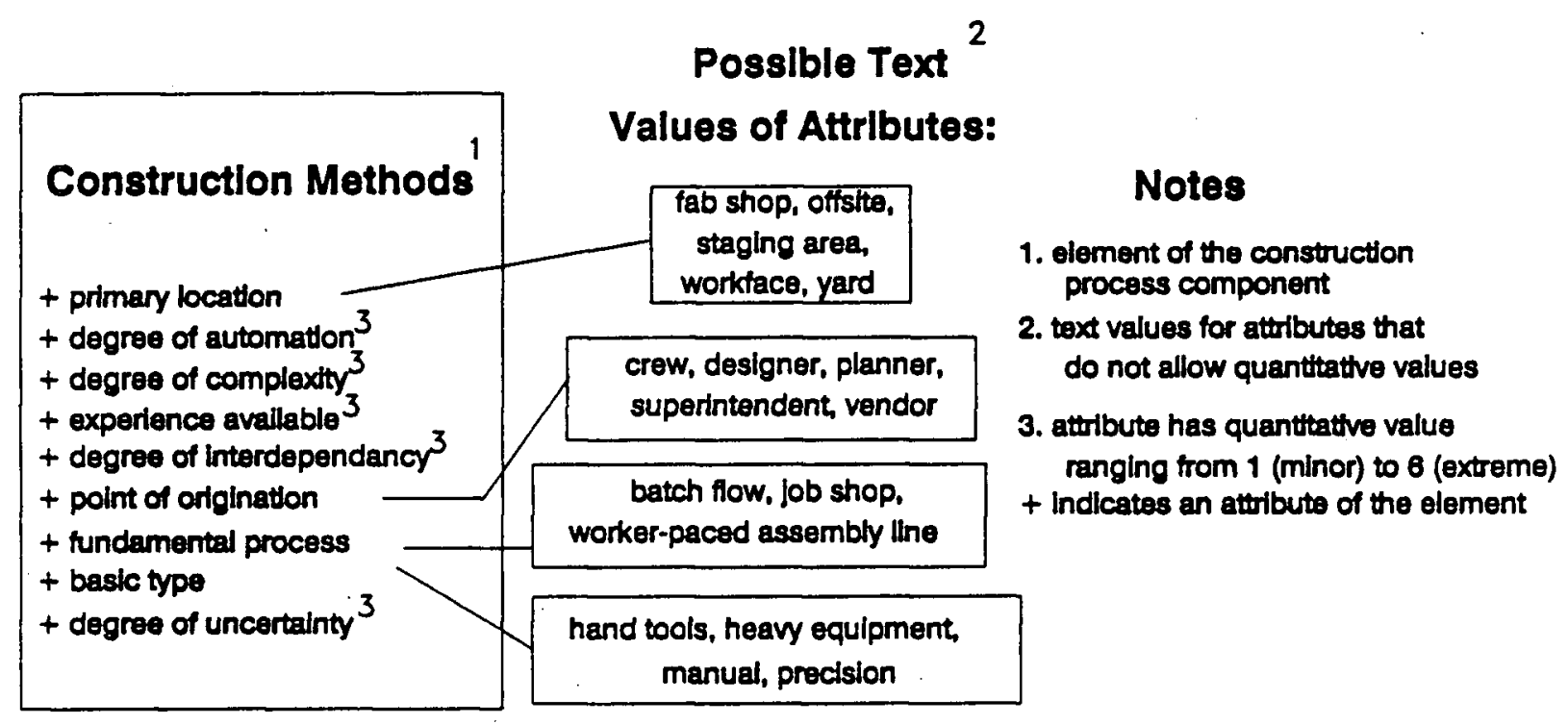

Figure 2.4 Example of Element, Attribute, and Value [from ratum 1988] 
Construction method has been defined as the manner in which resources on site are used to achieve specified forms of construction (Mansero 1987). In this reference, major elements identified as being part of the description of a construction method include:

1. a precise sequence of operations;

2. the relative pace of composite parts of operations;

3. interaction patterns with other operations;

4. construction plant;

5. expendable material and temporary works;

6. temporary services; and

7. craft skills.

Although many factors must be considered in plant selection, there are basic principles that can be used to confine this problem. For example, in building construction, a crane is considered to be a key resource for materials handling for major operations such as transporting forms, concrete, and other materials. For instance, Gray (1986) developed a systematic approach for the selection of a suitable crane for a high-rise building that embraces several factors. Among these factors are number and type of tower cranes versus mobile cranes, work load, required productivity rate, and crane work space and reach, given a finite number of available cranes. This provides a tangible 
example of structuring the knowledge pertaining to a complex problem of multi-task resource selection.

\subsubsection{Terminology Used in the Thesis}

This section presents the definitions of a number of terms that form part of the overall construction methods selection problem. They are elaborated upon in subsequent chapters. We take a restrictive view that the design of the permanent facility is fixed. Later, we acknowledge that contract provisions may consider other design alternatives or value engineering proposals. In addition, the requirements for temporary works, such as shoring and formwork, may become a significant design component.

Design Approach: Includes dimensioning the structure and its elements, and specifying the types of materials in response to loads, required functions, site features, regulations, and so on.

Construction Plan/strategy: Represents the high level abstraction of major aggregate activities that are sequenced in a pre-determined logical manner to realize a design. The estimate of an activity duration is dependant on the selected construction method and process. 
An example of a construction strategy in a high-rise building is to proceed excavation activity downward, simultaneously with constructing the super-structure upward, in contrast to the traditional bottom up construction.

Construction Process Model: Involves the interaction among equipment, labor and material, under physical and other project context constraints which characterize a major operation or construction cycle that is used in analyzing construction process. For instance, in a reinforced concrete high-rise building, concrete placement for the superstructure can be modelled using CYCLONE (construction process simulation program, see Halpin 1976) to measure its progress rate which indirectly indicates the whole project progress pace.

The construction process model combines design elements and construction resources according to a set of criteria. Thus, it could be used as a decision-making tool to rank, and accept or reject alternatives. Quantifiable measures for the model may include progress rate (productivity of the system), total cost, and duration. Qualitative variables may include the quality of work and safety during construction. 
Construction Method: Consists of a hierarchical assembly of design, strategy, resource, and process components that characterize a specific operation. For instance, a slurry trench wall (STW) method for a GWS encompasses the following components. The design elements include a specified slurry type and density, and concrete mix design (temporary and/or permanent facility). The construction strategy is bottom-up. The resources employed include all those involved in each operation, such as excavation, concrete placement, and retaining system installment. The process includes the arrangement of resource interaction, which constitutes a construction cycle. The construction cycle specifies a sequencing of the activities based on physical and resource interactions as well as other requirements imposed by the project context.

Construction Methods selection: A wholistic paradigm of methods selection consists of: suggesting a preliminary method which consists of design elements, construction strategy, and construction resources, and construction process; Designing a the method in terms of specifying its attributes; synthesizing a method by means of a model to predict its performance measures for the suggested method; Analyzing the performance measures versus expected goals, thereby accepting or rejecting a method alternative. 
"Recommendation" for a change in the method attribute values to alter a method that was rejected or to enhance method design effectiveness, is included in the Analyze operator. In essence, each element of the method is instantiated, and the resultant attributes values define the most suitable method based on project context variables. 


\subsection{Decision Making Model for Method Selection}

\subsubsection{Background}

Considerable effort has been expended in applying and adapting various operations research and systems analysis tools to the problem of construction methods selection. In general, the approach has been to show how a specific problem might be analyzed using optimization (Gates and Scarpa 1980, and 1984), queuing theory (Ringwald 1987), dynamic programming (Gaarslev 1977, Selinger 1980), simulation. (Halpin 1976, Ashley 1980), and so forth: i.e. given a construction related problem, here is how it might be analyzed.

Generalized approaches and definitions of methods selection which can be applied to a large class of problems, are the exception, not the rule. A notable exception is the work of Halpin (Halpin and Woodhead 1976, Halpin and Bernold 1986), in which he has attempted to develop a simulation approach for treating a broad range of problems. In this section we review previous work directed at improving one's ability to model and refine construction methods selection.

\subsubsection{Simulation Techniques}

Simulation can be used in planning and scheduling highly repetitive cycles in a construction project. A construction oriented simulation called CYCLONE was developed by Halpin 
(Halpin and Woodhead 1976), and later refined (Riggs 1980, Halpin and Bernold 1986), extended and integrated with other systems such as INSIGHT (Paulson et al. 1981 and 1987). CYCLONE is used for modelling at the construction operation level and is especially useful for predicting the behavior of a construction cycle design, given the required input data of durations and methods (Halpin 1976). Sensitivity analysis can be used to show the impact of each major variable on productivity.

Simulation applications in tunnelling are mostly used to predict the tunnelling advance rate, and cost breakdowns for major equipment items. Both deterministic and stochastic approaches have been used (Miller 1987 and Touran 1987). Performance measures for those methods are in terms of tunnel advance rate (feet/day) and total costs in general.

Knowledge Based Expert Systems (KBES) and simulation can be combined to form a computer aided decision making tool for modelling processes. O'Keefe (1986) has explored the Expert Systems-simulation taxonomy and its application areas. Suitable applications include intelligent front ends for simulation packages which provide advice on how best to formulate and interpret the results from a simulation model. For example, Bernold (1987) showed how heuristic rules may be used in conjunction with CYCLONE to evaluate construction process scenarios. 


\subsubsection{Decision Analysis}

Decision analysis is a term used to describe a body of knowledge and professional practice for the logical illumination of decision problems (Howard 1983). The decision modelling procedure follows three sequential phases: deterministic, stochastic, and informational. These three phases are repeated until the value of additional information is less than the cost of obtaining it. Decision analysis has been applied to methods selection (Gaarslev 1977, Ashley et al. 1979 and 1983). It is basically an analysis, as opposed to design tool. Ashley (1983) proposed crisis decision analysis as a tool to aid the manager to select the most appropriate alternative relying primarily on his experience and intuition. This methodology is built on two bases: a decision tree and political conflict resolution. This model is supposed to be utilized during construction at the strategic planning level. The tool, using project profit as the decision criterion, has been applied to select an alternative construction method for a hypothetical sewer tunnel during a crisis dealing with encountered surface settlement.

Ayyub and Halder (1985) proposed a decision analysis framework which considers information on relative risk, cost, benefits, and consequences of each construction strategy alternative. The decision criterion, safety of the 
construction operations as a function of a construction strategy, is measured in terms of the completed structure's consequent probability of damage. The main factors that affect safety are identified as being qualitative. The states of these factors are quantified using fuzzy set theory to estimate the risk of construction failures. The best construction alternative is the one with the minimum cost, with cost including initial cost of the structure under construction and expected cost of structure failure. The strength of this approach lies in treating linguistic terms. However, its weakness lies in its dependance on a single objective function (cost) based on safety factors only. Besides a decision analysis approach, decision making can employ other relatively new techniques including computer aided tools, such as decision support system (DSS). Since there is some overlap between DSS and ES, this subject will be investigated next.

\subsubsection{Decision support systems (DSS)}

DSS can be defined as a computer-based decision aid that provides convenient access to decision models dealing with production, distribution, financial analysis, and so forth (Blanning 1984). Both DSS and ES incorporate features of management informations systems (MIS) and operations research (OR) techniques. 
Turban and Watkins (1985) contrasted differences in approach between DSS and ES, and examined the possible connections between the two systems and the benefits of their integration. A major difference is that an ES makes, rather than supports, decisions and contains a judgmental model rather than a causal model. Furthermore, an ES offers conclusions along with supporting justification or an explanation using transferred expertise. A DSS helps a user evaluate and choose among alternatives based on the system model, mainly using the user's judgement and discretion. One can loosely think of a DSS as a quantitative (causal) modeling approach to a problem, whereas an ES is regarded as a qualitative (judgmental) and quantitative modeling approach.

Dss for Methods selection: Within the construction industry, Mansero and Chapman (1987) argued that DSS provides the best means for methods selection for reinforced concrete structures rather than an ES, the output from which would be too prescriptive. They proposed a DSS system to model alternative ways of providing in-situ reinforced concrete framed buildings, with particular emphasis on selecting suitable formwork methods from a construction planning perspective.

Kim (1984) proposed and implemented a DSS to select an adaptable tunnelling method (which adapts construction 
methods to encountered geological conditions) to optimize the design/construction methods selection for tunnels in rock. In his work, he deals with construction methods for excavation and wall support, including selection of equipment. He proposes a framework for generating decision support information in adaptable tunnelling and then derives analytical methodologies for the proposed DSS, employing stochastic dynamic programming (DP) algorithms. A relative confidence level is used as a measure for ranking competing alternatives. Decision variables considered are excavation and support methods. State variables are geological conditions, and cost/time factors (productivity in terms of advance rate, equipment and material costs, and overhead costs). The objective function selected is expressed in terms of stochastic variables describing geological conditions. Cost/time factors are treated as deterministic with constants derived from previous tunnelling projects. The objective of the optimization framework, in adaptable tunnelling, is to identify the most cost-effective chain of excavation and support methods, each of which is technically feasible. At the same time, each must be economically feasible for the anticipated geological conditions in its subsequent tunnel segments (i.e. the tunnel is divided into equal segments to reflect the changing geological conditions along the tunnel). 
The objective function (total construction cost), is subject to two constraints. The first, technical feasibility, refers to the ability to employ an excavation method suited to the encountered ground conditions and a support method that satisfies structural and functional criteria. The second constraint, economic optimization, refers to employing the most cost-effective combination of different methods along the tunnel, given geological variations.

The features of Kim's work that are of direct relevance to the thesis work described herein are:

1. Planning decisions are divided into a preconstruction and a construction phase.

2. Output decision variables, for the preconstruction and construction phases, include feasible types of combined construction methods, level of confidence in selecting a method (the major criterion for ranking alternatives), expected loss for each method as an upper limit for the additional geotechnical exploration expense, and total cost and time for each alternative.

3. Input state variables encompass geological conditions and cost/time data for each method. Costs are defined as cost of equipment, material, labor, and mobilization/demobilization charges for methods changes. Time data include the productivity of each method versus a ground class, method change duration, and lead time as predetermined data.

4. Variations of a construction method are treated as discrete methods. For instance, the model has been applied to select combined methods among five method variations of drill and blast excavation/support alternatives, as opposed to 
competing with other major methods such as tunnel boring machine, or partial face boring.

5. The model does not consider important project attributes such as physical constraints, material handling, ground subsidence, and ground water control.

Law (1987) proposed a conceptual DSS for the detailed design of construction activities associated with projects characterized by significant repetition. High-rise building was selected as the problem domain. A Work Breakdown structure (WBS) was used to decompose activities into smaller work operations in order to select appropriate construction technologies. The attributes of construction alternatives consist of productivity, equipment and material unit costs, and crew makeup. The suggested data structure, see figure 2.5, does not incorporate some important attributes such as the technical feasibility of those methods in terms of project physical constraints, flexibility of the equipment used, and the level of confidence associated with each method.

Law indicated, in the problem recognition section, that in the design of the construction activities, field engineers have to draw on their experience with similar previous projects to apply their knowledge of construction method (CM) selections. Such knowledge resides with a few key personnel and is rarely documented. It is obvious that the DSS approach for methods selection addresses the quantitative aspects of the problem. Law stressed the 
importance of modelling the qualitative or judgmental part of construction methods assessment and selection.

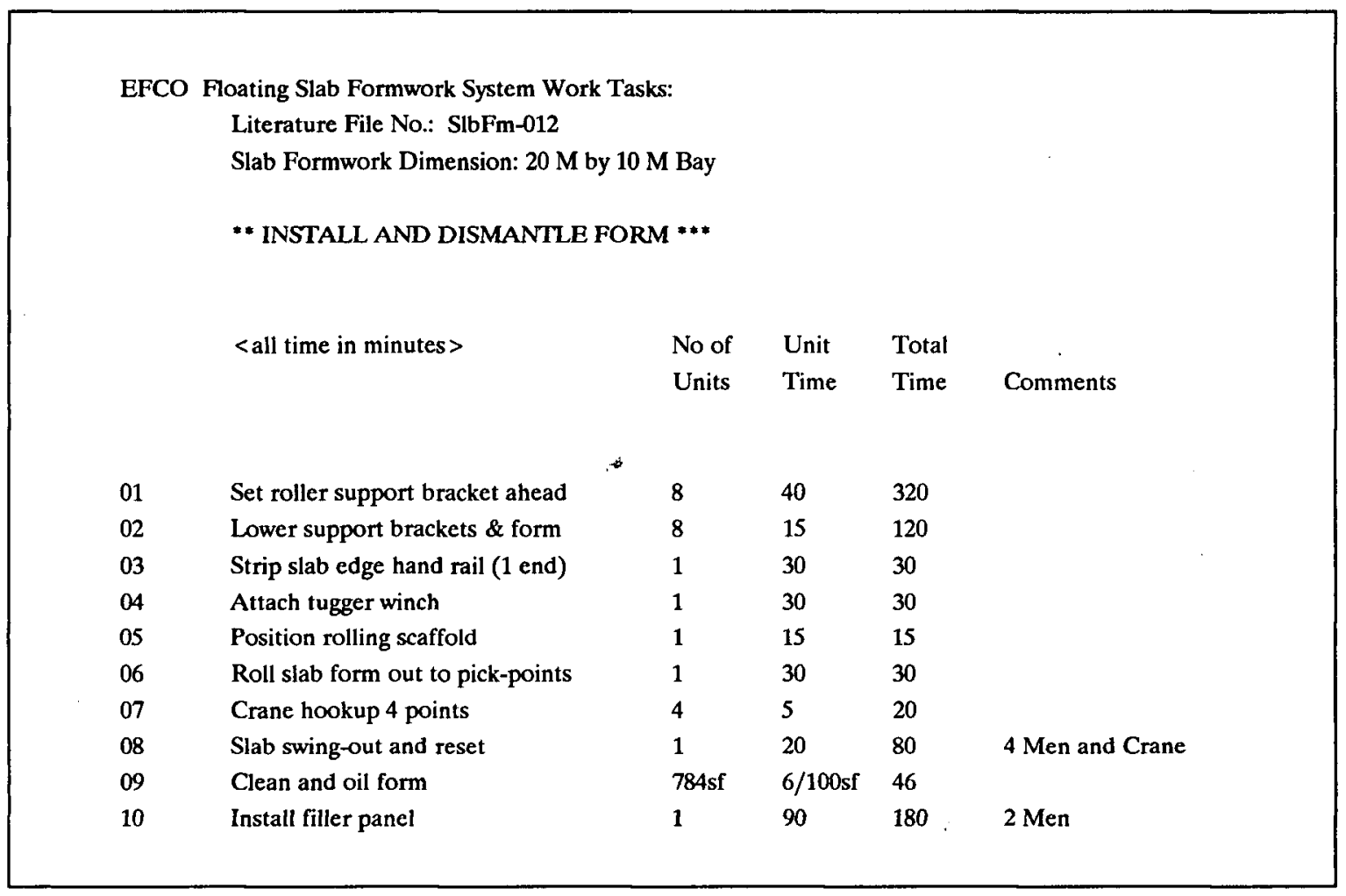

Figure 2.5 suggested Data structure for Selected Technology [from Law 1987]

\subsection{Knowledge-Based Expert Systems}

In this section, we identify the main components of a Knowledge Based Expert system and identify relevant work in the construction domain, especially methods selection, that relates to each component.

Modelling of uncertainty in knowledge has not been explored -- existing uncertainty methods include probabilistic methods (Bonissone 1985, Duda et al. 1979), 
confirmation theory (Buchanan and Shortliff 1985), fuzzy set theory (Zadeh 1975) and Dempster-Shafer theory (Shafer 1976). While relevant to construction management problems, it is outside the scope of this thesis. However, the uncertainty in terms of outcomes of site conditions (especially of geological conditions) was treated.

Gashing (1985) defines KBES as an interactive computer program incorporating judgement, experience, rules of thumb, intuition, and other expertise, to provide knowledgeable advice about a variety of tasks.

\subsubsection{KBES components}

A typical KBES has four major components; a Knowledge Base, consisting of Knowledge Representation and Acquisition; an Inference Engine; a Context; and an Explanation Facility.

1. Knowledge Base (KB)

The knowledge base (KB) contains a symbolic representation of expert rules of judgement and experience in a form that enables the inference engine to perform logical deductions upon it. Such facts and rules are specific to the domain of the problem. Difficulties in developing a KBES are attributed to knowledge representation and knowledge acquisition.

Knowledge Representation : Knowledge Representation (KR) is the set of syntactic and semantic conventions used to encode 
the facts and relationships that constitute knowledge in a knowledge based system (Winston 1986). Experience in developing KBES has shown that a robust, yet precise knowledge representation is often the key to avoiding superficiality or shallowness in the solution of realistic problems (Jackson 1986). Selection of a KR technique is a fundamental step in the application of ES to a problem. The $\mathrm{KR}$ process is concerned with the problem of encoding the knowledge so that it can be easily manipulated by the computer. In general the following elements must be represented: domain terms which deal with the language or jargon used by the expert in the field; structural relationships which treat the interconnections of compound entities; and causal relationships which deal with causeeffect relations between components.

$\mathrm{KR}$ techniques may include production rules (Buchanan and Shortliffe 1976), predicate logic (Clocksin and Mellish 1981), semantic nets (Minsky 1968, Duda et al. 1978), frames (Minsky 1975), and object oriented programming (Bobrow and Stefik 1983, Goldberg 1981).

Knowledge Acquisition : Knowledge Acquisition (KA) is the transfer, and the transformation, of problem-solving techniques from some knowledge source to a program (Buchanan and shortliffe 1985). The major bottleneck in building an 
$E S$ is the scarcity of knowledge engineering skills to interact with one or more human experts.

Several methods are used in the KA process. They include the use of unstructured interviews, structured interviews, prototype system development, rule induction, observation, and finally machine learning of rules (Gruber and cohen 1987). These methods embody theories and knowledge from computer science, psychology, linguistics, and sociology, in addition to technological expertise.

\section{Inference Engine}

The inference engine (IE) is the part of a KBES that contains the general problem-solving knowledge and is characterized by strategies which draw inferences and control the reasoning process (Mikroudis 1986). The IE guides the development of a solution using the facts and rules stored in its $\mathrm{KB}$ and the information it acquires from the user. Thus, the IE is used to derive new facts from known facts and to regulate the order in which reasoning occurs. IE strategies used to make inferences include, but are not limited to, modus ponens, resolution, and inheritance. Control strategies include backward chaining, forward chaining, agenda control, mixed, and others (Charniak and McDermott 1985). 


\section{Context}

The context is a temporary data storage in which known and deduced facts are stored during a consultation session. The context builds up dynamically during the solution process of a particular problem. It is used by the inference engine to determine the next step in the process. Data may come from, or go to, an extended data base, analysis/design programs, or even data acquisition devices. The inference tree provides a further mechanism for representing hierarchical relationships and for assigning values to object slots by instantiating.

\section{Explanation Facility and others}

The explanation facility (EF) component serves to partially trace the ES reasoning process in order to justify the conclusions made during a consultation.

The two widely used commands are HOW and WHY. For a network of goals, rules, and hypotheses, How asks what rules were involved in solving the problem. WHY asks for the reasons some information is requested by the system. In a goal driven ES, the HOW rule propagation direction is from goals to the initial states (backward chaining). The WHY direction is vice versa (forward chaining). 


\subsubsection{Expert systems for Construction Management}

Construction engineering and management involves many complex decision-making problems, such as resource allocation, planning and scheduling, safety, analysis of construction processes, and productivity measurement and improvement. The solution of these are highly dependant on engineering and trade judgement, rules of thumb, and subjective evaluations.

As stated in the previous section, construction management decision-making tools have traditionally employed quantifiable models (networks, OR techniques, etc.). Their strength lies in their rigorous analysis of the available data culminating in an optimal, or near optimal, solution to the problem. Their main weakness is the total dependance on the quantitative data necessary to represent the various relationships that describe the problem, many of which are imperfectly understood (Warszwaski 1986).

When construction management decisions involve more qualitative information or relationships (experience, judgement, and intuition), or when multiple decision criteria are present, the traditional approach is of limited use, being more at the tactical than strategic level. Such limitations can be overcome to a certain degree by incorporating the experience, heuristics, and judgement of acknowledged experts into an ES. 
Since 1984 several papers, articles, and conference proceedings have been published that provide an overview of ES applications in Construction Management (Wager 1985, CIB86 1987, Levitt 1987, Mohan 1990), and suggest further applications in this field (Chin 1987, Mohan 1990).

Reviews of current ES applications have been reported recently (Wager 1986, Levitt 1987). Applications identified include:

1. construction project organization design (Zurich, Switzerland) ;

2. time estimating systems (Civil \& Civic Australian construction firm);

3. repetitive construction risk analysis (University of Texas) ;

4. decision making and risk analysis (Georgia Institute of Technology);

5. intelligent construction risk identification systems (University of Texas);

6. layout of temporary construction facilities (Stanford University);

7. evaluation of project personnel based on progress data from project time/cost monitoring systems (MIT) ;

8. vertical construction planning/scheduling (University of Illinois, and CMU);

9. project planning and control (Stanford University, Levitt et al 1988);

10. construction project monitoring (CMU);

11. maintenance advisor (PTY Ltd, Australian elevator construction and maintenance contractor); 
12. equipment and plant selection (University of Technology, Loughborough, U.K.).

other applications have been suggested by Trimble (1987), Warszwaski (1985), Chin (1987), Mohan (1990) as follows:

1. design synthesis and interpretation of building code regulations;

2. estimating procedures and cost control;

3. the analysis and evaluation of construction scheduling;

4. selection of appropriate plant and equipment;

5. site planning;

6. construction financing;

7. design and construction planning of prefabricated buildings :

8. quality control;

9. safety practices;

10. contractual claims analysis; and

11. evaluation of alternative construction methods at early design stages.

\subsubsection{KBES for Construction Methods Selection}

Research work on the use of expert systems for methods selection has been tailored to specific applications. Generally, such systems can be classified as rule based, or frame and/or object oriented.

Within the first category, some applications are directed at selecting a key resource, particularly equipment for a specific job. Gray and Little (1985) examined the influence of craneage resources required to lift large units in a 
high-rise building and the effect of multiple crane resources on the activity duration calculations. Subsequently, an expert system for craneage resource analysis was developed to select and locate the most desirable crane on the site for a high-rise and low-rise building construction. Later, this expert system was imported into a more comprehensive planning rule-based expert system directed at determining all work activities implicit in the design of a high-rise building (Gray 1986).

Activities were defined according to type of work (resource labelled: material, trade, plant), operationally significant function (direction of movement: vertical or horizontal), and operationally significant location (grouping activities of different sequence and size).

Components of an activity's duration are work volume and resource level. The work volume is set by the design. Resource level is variable. Resources are set in fairly coarse groups, either gang or piece of plant, at the minimum level consistent with normal practice. Rules and heuristics were used to select an activity, link activities (precedence, and time links), and perform problem solution processes. This application shows how expert systems can be used to encode algorithms (network analysis), and heuristics for activity selections. 
In the same category, other applications include selecting a crane type (tower crane versus mobile crane) and size for high-rise building construction (Harris and wijestundera 1987), and scraper equipment for road earthmoving, given the specific project conditions (Harris 1988). The former authors concluded that selecting construction equipment is largely based on uncertain and intuitive knowledge, allowing only broad rules of thumb to be formulated. Moreover, they suggested that further applications should include output data and production information pertaining to plant and labor resource evaluation. In both examples, the knowledge base is largely heuristic in nature, and knowledge acquisition was essential for deriving the inference for problem solution. Also, in those applications, methods were represented as equipment for capital intensive projects.

The second application category, frame based expert systems, includes work done by Logcher and Nay (1985), Kunz et al. (1986), and Hendrickson et al. (1988).

Chief among those applications is construction planex (Hendrickson et al. 1988). Planex, a KBES for construction planning, is used to plan modular high-rise buildings, including excavation, foundation, and structure construction. Planex starts with a design alternative as input which consists of several design elements (a footing, 
column, and beam for a modular reinforced-concrete building). The design element generates construction element activity frames (i.e excavation, pouring concrete, etc). Figure 2.6 shows a sample element activity frame created to describe the excavation activity required for a footing. The element activity is identified by a code number using the extended MASTERFORMAT code. The first six slots define its designation and relevant parents of design elements and project activities. These are followed by slots for amount of work, unit-of-measure, crew, materialpackage, duration, and successor element activities. The crew attribute has been evaluated to excavation-foundation05.

\begin{tabular}{|c|c|}
\hline \multicolumn{2}{|c|}{$\begin{array}{c}\text { Element-Activity } \\
\text { p01-c00-b00-100-ca-02-220-10-01 }\end{array}$} \\
\hline SLOT & VALUE \\
\hline is-a & ea \\
\hline ea-name & excavation-column-footing-01 \\
\hline ee-code & $01-220-10-01$ \\
\hline Qa-ot-DE & p01-800-600-100-do-60-01-01 \\
\hline parent-EA & $p 01-500-600-100-88-01-220-10$ \\
\hline QQR-OPPA & p01-200-b00-100-pa-10-60 \\
\hline amount-ot-work & 24.0 \\
\hline unit-of-measure & culyd \\
\hline crow & excavation-foundation-05 \\
\hline material-packege & none \\
\hline duration & 16 hours \\
\hline successors & $p 01-500-b 00-100-0 a-02-220-10-02$ \\
\hline
\end{tabular}

Figure 2.6 sample Element Activity Frame [from Hendrickson et al 1988]

After element activities have been created, they are grouped into project activities based on selected 
technologies. The activities are then sequenced and their duration estimated in order to develop the schedule.

The knowledge base is organized into a set of knowledge sources that represents rules, heuristics, and calculation functions. Decisions and computations undertaken during the planning process can be stored in any of the frames in the Planex hierarchy of frames and can be inherited upward and downward between design element, project activity, and activity element frames. When frames are created by the operator modules, relevant knowledge sources in the knowledge base will be evaluated. Within a sequence of operations applied to create a construction plan, the selected technology operator uses heuristics related to soil and site information, resource productivity information and other factors, by activating relevant knowledge sources (KS) designated as $\mathrm{kS}$-technology-xx-xx, to group element and project activities under an auxiliary group object that is used to store the common technology choice. Figure 2.7 shows an example of a knowledge source, namely KsTechnology, for selecting excavating equipment pictured as a decision table, whereas it is actually encoded as frames and production rules. This kS contains two project context conditions, three rules, and three actions. The second condition of "Rs-water-level" is an embedded knowledge source that has to be evaluated first. The third rule 
indicates that if none of the previous two rules were fired, the appropriate technology is "special machine".

\begin{tabular}{|c|c|c|c|c|c|c|}
\hline \multicolumn{7}{|c|}{ KS-Technology-Example } \\
\hline Object & Slot & Op & Value & \multicolumn{3}{|c|}{ RULES } \\
\hline \multirow[t]{2}{*}{ soll-characteristics } & \begin{tabular}{|l|} 
soil- \\
type
\end{tabular} & Is & hard & true & false & not fired \\
\hline & $\begin{array}{l}\text { KS-water- } \\
\text { level }\end{array}$ & Is & wet & $\mathrm{fa} / \mathrm{se}$ & ture & not fired \\
\hline \multicolumn{4}{|c|}{ U } & $\gamma_{\text {then }}$ & then & $7_{\text {then }}$ \\
\hline \multicolumn{3}{|c|}{ power-shovel } & & selectod & & \\
\hline \multicolumn{3}{|c|}{ clamshell } & & & selected & \\
\hline \multicolumn{3}{|c|}{ special-machine } & & & & selected \\
\hline
\end{tabular}

\section{Figure 2.7 Example of Rnowledge Bource \\ [from Hendrickson et al. 1988]}

According to the inference strategy implemented in Planex, element and project activities for the excavation are supposedly selected and assembled based on the plant selected from the ks-technology instantiating shown in figure 2.7. Task durations are estimated from decision tables and calculating rules in a manner similar to that used in MASON system (Hendrickson et al. 1987). Precedences among element activities are also determined and recorded in slots of the element activity frames. These precedences can be of two types: physical or resource-related. 
For the Planex system, the authors indicated that determining the equipment to be used, the number of crews or pieces of equipment, inter-task precedence and task duration, involve diagnosis and prediction as contrasted to synthesis, in activity definition. This example shows that the way in which a method, mimicked as equipment selected based on project context, is used to combine element and project activities among numerous alternatives, is essential in constructing the planning schedule.

Logcher and Nay (1985) described a conceptual expert system for analyzing construction project risks. Hierarchical frames were used to represent project tasks, work packages, risks, resources, and additional siterelevant information. Figures $2.8,2.9$, and 2.10 show a sample of the labor, equipment, and process frames that are to be created during a session. Their values will be inherited by Work Package and Review Data frames for further manipulation of the project risk analysis. The frames presented show how construction related concepts could be represented by describing and detailing their attributes.

In this application, frames were found to be a knowledge representation strategy capable of capturing relevant problem characteristics, while rule-directed inference was used to associate project risks with work packages. 


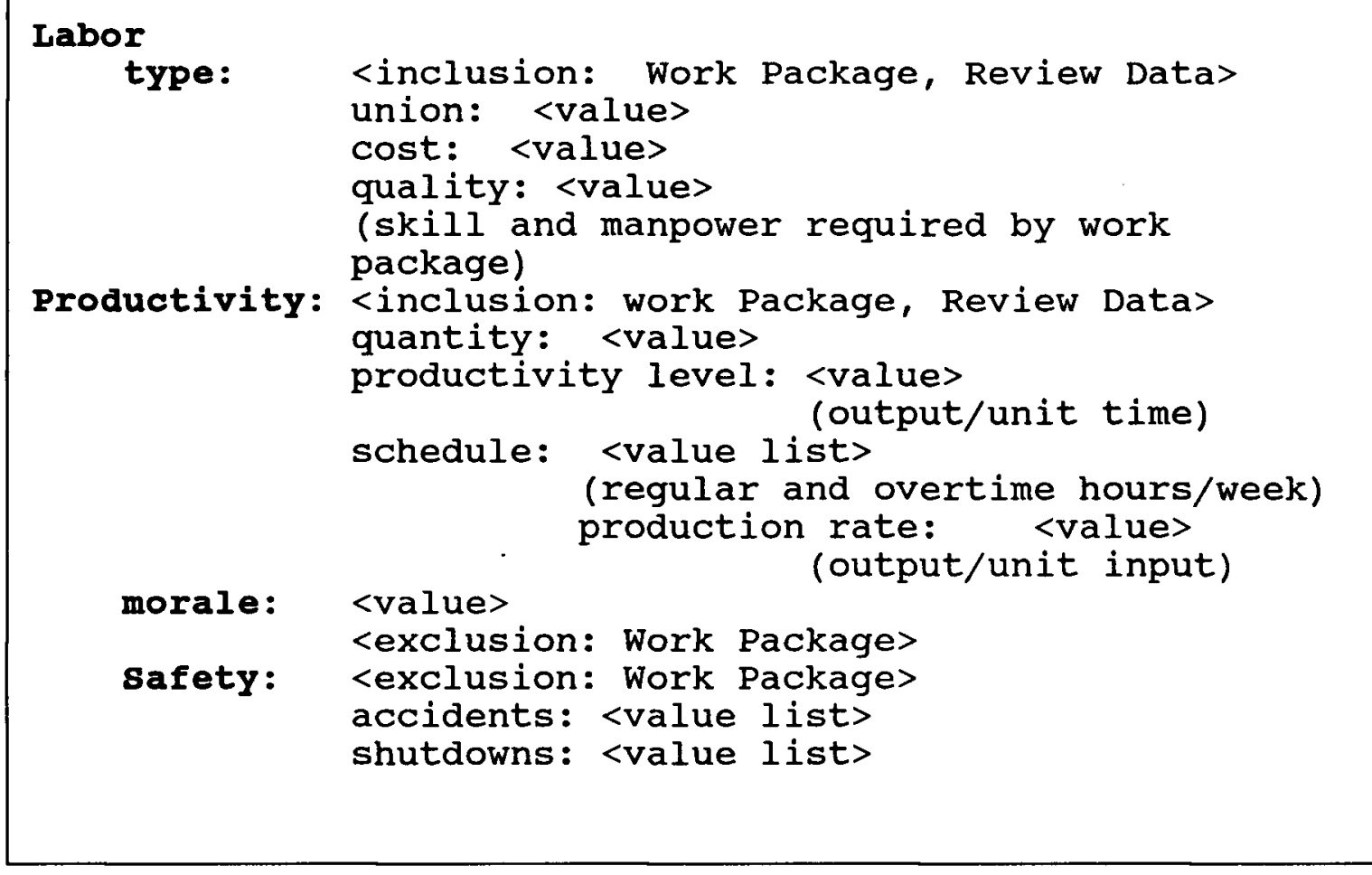

\section{Figure 2.8 Labor Component Frame [from Logcher and Nay 1985]}

The foregoing expert systems sample applications show the diversity of the methods, selection literature. From the thesis viewpoint, each example addresses specific instances of methods selection. There is a lack of a wholistic scheme for specifying and analyzing a method. The literature has showed how some expert systems have been incorporated and/or evolved into a larger intelligent system for planning, site layout, etc. In the same vein, it is conceivable to envision a generic tool that consists of a series of ESs, small and big, that are tied together to select and specify the most suitable method. 


\section{Equipment}

type: general specification: <value list> description: <inclusion: Work Package, Review Data> date information supplied: <value> supplied by: <value> equip name: <value> rated capacity: <value> alternative equip type: <value> operating hrs. until mainten.: <value> source: supplier: <value> <inclusion: Work Package, Review Data> <domain: Owned, Leased, Owned by subcontractor>

cost: <value>

<inclusion: Work Package, Review Data> quality: <value> <inclusion: Review Data>

Productivity: <inclusion: Work Package, Review Data> production rate: <value>

Reliability: <value> <inclusion: Review Data>

Figure 2.9 Equipment Component Frame [from Logcher and Nay 1985]

Process

<inclusion: Work Package> work package complexity: <value> company experience: <value> contractor: <value> experience: <value> 
As a guide to general topics for KBES based method selection, areas of research interest include configuration of crews; choice of construction methods; man-machine tradeoffs; choice of transportation modes for the movement of materials, personnel and equipment; selection of optimum sizes, configurations, and methods for joining various components in modular construction; and deep-excavation problems (Mohan 1990).

of particular value, is the work done in the field of industrial engineering, specifically in manufacturing, which provides useful ideas for the definition of the methods attributes outlined in chapter 4. Those include a KBES approach for modelling a plant production plan (Reddy and Fox 1982), specification and design of flexible manufacturing systems (Mellichamp and Wahab 1987), creation of intelligent environments embodying simulation and artificial intelligence, applied to simulation (Shannon 1986 and 1987), and automation of the model construction phase of the simulation life cycle (Murray and Sheppard 1988). 


\section{Cut-and-Cover Methods in Soft Ground}

\subsection{Introduction}

A Cut-and-Cover tunnelling project has been selected as the problem domain in which to explore the methods selection problem because of its accessible knowledge base and established alternative methods.

The goals of this chapter are to give the reader an example of Cut-and-Cover tunnelling, expose empirical and procedural knowledge for the design and construction of related ground wall support systems, and identify the kinds of decisions relevant to the methods selection problem.

A general introduction to the tunnelling domain is given in section 3.2. Section 3.3 covers the Cut-and-Cover tunnelling alternative in more detail including its characteristics. Section 3.4 reviews GWS alternative design and construction procedures; in particular, steel sheet piles which is used as the actual project context for the CMSA development.

Section 3.5 covers the excavation operation briefly, since it is incidental to GWS retaining system design variables. Section 3.6 examines the factors influencing the selection of cut-and-cover tunnelling alternatives.

Section 3.7 provides a summary of a hypothetical soldier pile and lagging cut-and-Cover tunnelling example, based on 
a real project, highlighting project context goals, constraints, and construction cycle decision variables. This example serves as a primary source for subsequent variations of cut-and-Cover tunnelling throughout the thesis that are applicable to traditional (top-down) Cut-and-Cover tunnelling.

\subsection{Tunnelling Background}

Tunnelling can be defined as the construction, by any method, of a covered cavity of pre-designed geometry whose final location and use is under the surface (Bickel and Kuesel 1982).

Costs of tunnel construction include fixed and variable costs. Fixed costs are a function of design features, such as location, grade, types of wall support and final tunnel structure, and the cost of special equipment, such as tunnel boring machines, including mobilization. Variable costs are a function of time, labor, utilities, supervision, and equipment.

The two major approaches for constructing tunnels are underground tunnelling, such as using a tunnel boring machine (TBM), New Austrian Method, drill and blast, and Cut-and-Cover tunnelling. Both approaches employ different design elements, construction strategies, construction resources, and construction process model. The decision to 
choose one approach over the other depends on costs including environmental and social costs, as well as project parameters such as duration and geotechnical factors.

Figure 3.1 shows a comparison between costs of both construction approaches. This figure highlights the wide variance of both tunnelling alternatives costs, which is context sensitive especially because of the uncertainty of the geological conditions. It may appear from the figure that the cost of boring tunnelling excavation is more than Cut-and-Cover excavation; however, their total costs might be the same. For shallow tunnels, the direct cost of this Cut-and-Cover method is likely to be much less than the cost of the tunnelling method. However, environmental and social costs can change the balance completely. With increasing depth, the direct cost of the trench excavation and temporary wall support system increases rapidly. 


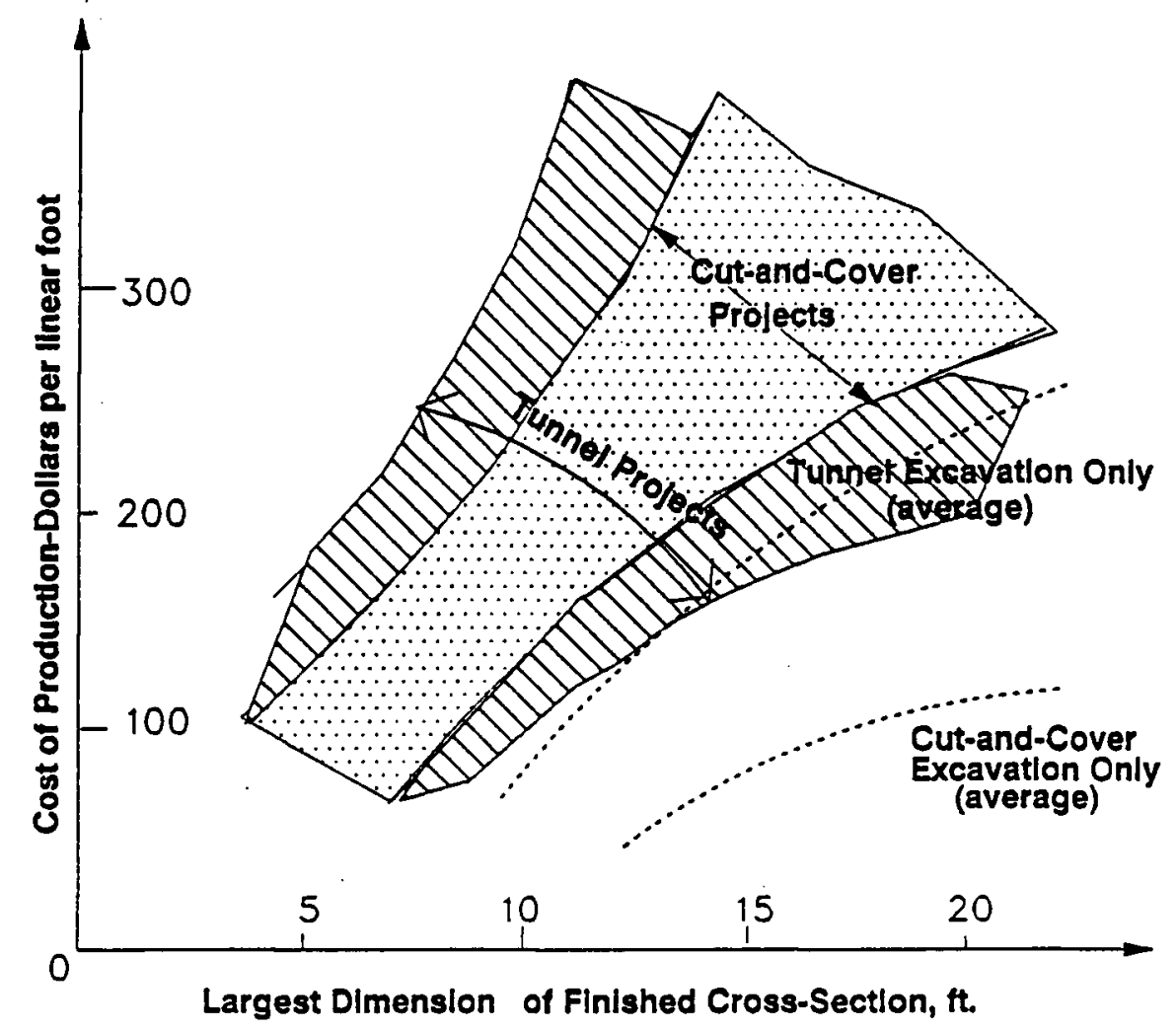

\section{Figure 3.1 cost Comparison for Tunnelling Alternative versus Cut-and-Cover Alternative [from Blokel and Kuesel 1982]}

The dimensions of the tunnel depend on tunnel use and ground conditions. For instance, the width of the underground structure for a highway is normally 35-65 feet, while for a rapid transit structure, it is 35 feet. The depth below ground varies from 10 feet to 100 feet.

\subsection{Cut-and-Cover Tunnelling Alternatives}

\subsubsection{Background}

Based on the excavation method employed, cut-and-cover tunnelling can be categorized into traditional and Milano approaches. Each one embraces different method attributes 
of design elements, construction strategies, resources, and so forth.

Before we describe these alternatives, we clarify the meaning of some of the terminology. First, a construction strategy is a function of physical structure (design elements) and project context (design features, site features, contractual provisions, regulatory environment, prevailing economic conditions and so forth) which entails the logical sequence of a set of activities. Each activity has several attributes: a work scope (amount of material to be added or removed), a construction technology, a duration, etc. An activity could be executed by means of operations which break it down into smaller tasks that could be achieved by one or multiple resources, i.e., an operation designates a grouping of resources utilized directly or indirectly to construct the design elements. A variation of task/resources make-up may produce several alternative operations. For instance, a soil stabilization activity is not required for the slurry trench wall design element while it is required for the soldier piles and lagging design element under a given project context. For the latter case, a soil stabilization alternative operation could include soil freezing, micro-piling, cement grouting, etc.

Note that a construction strategy label could be a misnomer. For the two types of Cut-and-cover tunnelling, 
the top-down and bottom-up (Milano) construction strategies refer mainly to the excavation progression that distinguish them. Next, we briefly describe the two approaches.

\subsubsection{Traditional Cut-and-Cover Tunnelling (Top-Down)}

Excavation is the major activity to distinguish this approach from the other. A top-down construction strategy is used to designate this approach since it implies that excavation proceeds from the ground surface to the bottom of the tunnel.

For the traditional cut-and-cover tunnelling strategy, the operations involved and their sequencing are excavate and support the trench simultaneously, construct the tunnel structure, backfill the trench and restore the surface.

\subsubsection{Milano Cut-and-Cover Tunnelling (Upside-Down)}

A bottom-up or upside-down construction strategy refers to the fact that the excavation proceeds from the bottom of the tunnel to the ground surface. This method is relatively new and associated only with the ground wall support system design element alternative of slurry trench wall (STW), although it could be done by top-down excavation as well. STW and other GWSS techniques will be reviewed later in section 3.4 .1 . 
The upside down or, so called, Milano strategy provides an alternative construction strategy which speeds up traffic restoration. Instead of excavating to the full depth using temporary bracing, permanent steel may be installed as the excavation progresses. The major activities include:

1. construct STW,

2. excavate to the roof slab elevation,

3. construct the roof and use it as permanent bracing,

4. start backfill, and/or

5. excavate below the roof, and/or

6. construct base slab (invert) and complete the structure.

3.3.4 Major Operations Common to Cut-and-Cover Tunnelling Major operations during the construction phases cut-andCover tunnelling are decomposed into the following categories:

1. stabilizing surrounding structures (underpinning),

2. ground stability,

3. ground water control,

4. ground wall support installation,

5. excavation,

6. permanent structure installation, 
7. utility relocations, backfilling, and compaction.

Each of the above operations employs different construction procedures (resources and construction process model) subject to the interactions between them. For example, the pace of excavating both horizontally and vertically is bounded by the shoring retaining system spacing that provides structural stability for the GWSS during construction.

To give a flavor of the size and complexity of construction method attributes, consider table 3.1. It provides a subset of method attributes including variable design elements for the temporary GWS, fixed design elements of the permanent tunnel structure, and alternate operations. Table 3.1 is set up so that each operation is independent and has several alternatives. Therefore, each combination of those attributes, vertically in the table, may represent a feasible alternative to a method -embracing design/construction attributes. Hypothetical criteria are provided to rank feasible alternatives based on one criterion or a mix of criteria. For instance, consideration of multiple criteria could be used to select Alternative (3) as the most favorable. 


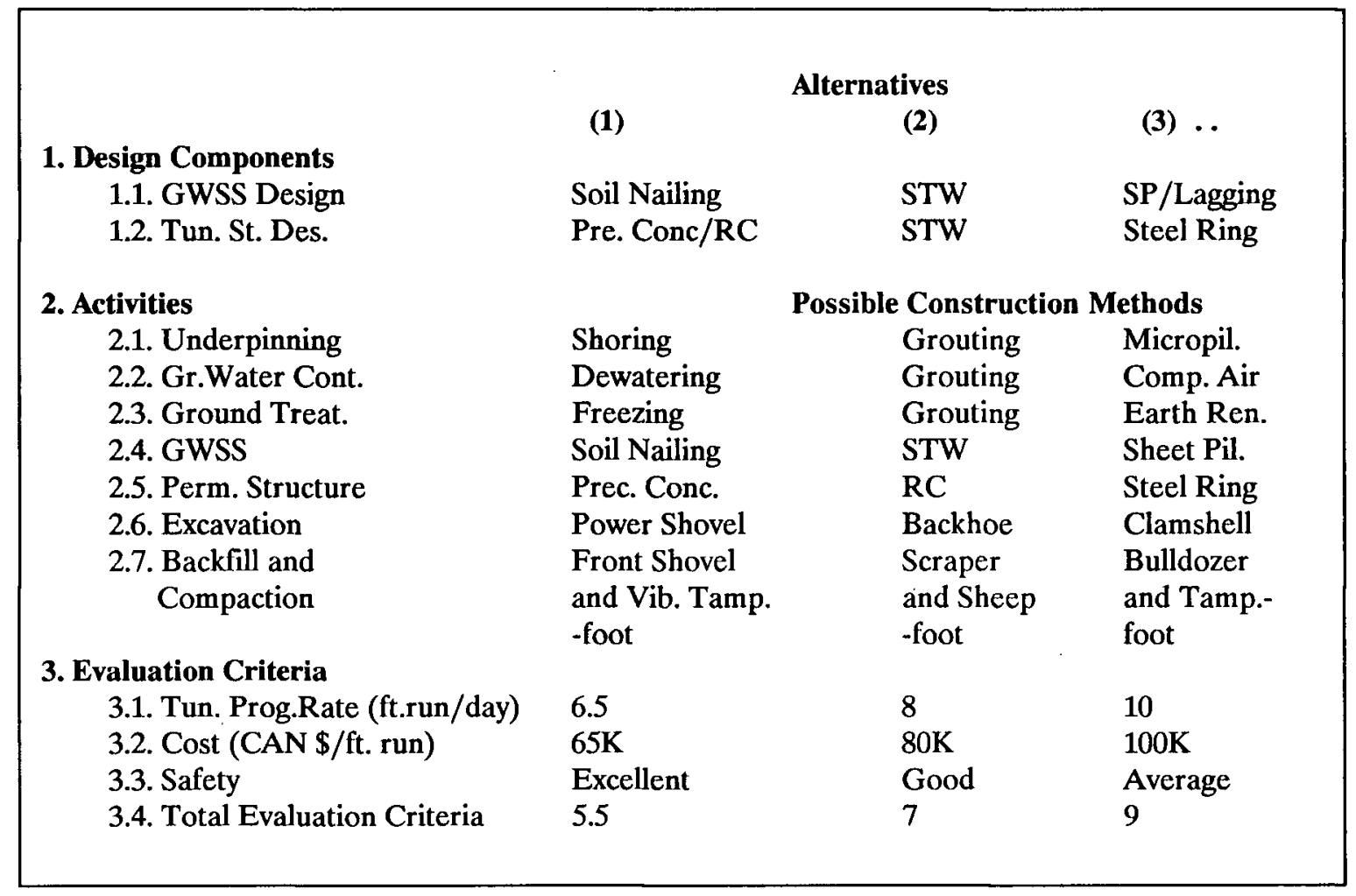

Table 3.1 Partial space of Design/Construction Elements for Cut-and-Cover Tunnel

It is useful to analyze the cost breakdown of the structural aspects of a cut-and-cover tunnel project in order to identify the most important cost components in the construction process. Shen (1981a) estimated that approximately $60 \%$ of the cost is directly associated with the cost of the temporary ground wall support system structure and excavation. $80 \%$ to $90 \%$ of the cost of building the permanent tunnel structure is influenced significantly by decisions about the type of ground wall support system, including shoring system. Thus, any significant cost saving in the GWSS will result in a 
substantial lowering of total project cost. It is therefore important to examine GWSS design and installation, and excavation operations more closely. The former will be treated as a separable construction process with its associated resources, and the latter will be treated as a method that embraces design elements and construction resources, while construction strategies and processes are implicitly discussed.

Next, we examine common GWSS alternatives and treat their design features and construction procedures. Such procedures exhibit the analytic and heuristic knowledge required for construction method attributes synthesis. Some of those procedures will be utilized in the CMSA prototype development in chapters 4 and 5 .

\subsection{GWSS Alternatives}

The term GWSS, ground wall support system, is taken to mean temporary and/or permanent support walls and the shoring schemes used to retain them. Alternatives for temporary (removable) and permanent support systems are as follows:

\section{Temporary support systems}

1. Soldier Piles with Lagging;

2. Sheet Piling (steel, timber, or concrete) ; or 3. Soil Nailing.

2. Temporary/Permanent support systems 
1. Slurry Trench Wall (cast in place, precast);

2. Secant piles (cast in place, precast); or

3. Any combination of the above.

Alternative retaining systems include:

1. Wales and struts or rakers;

2. Compression rings (when excavation is relatively small in plan); or

3. Tieback anchorages.

In deep but narrow excavations, the preferred retaining system is generally struts and wales, whereas for wide excavation areas, cross bracing and rakers, or similar shoring systems, are employed. Tie backs are popular in deep and wide cuts to provide obstruction free excavations (Winterkorn and Fang 1975).

other GWSS methods such as freezing and grouting might be used as the wall support system; however, they are not used frequently due to their high cost. They may be required, nevertheless, for localized water control or treatment. GWSS methods combined with stabilization methods are used to control soil and ground water movement during or after construction. Such movements may often cause settlement and damage to excavation support and adjacent installations.

The design of the GWSS depends upon the ground conditions and the importance of preventing surface settlement. settlement is caused by movement of the soil surrounding the 
excavation as a result of bottom heave, inward movement of the GWS or loss of ground through the GWSS.

To evaluate the need for underpinning adjacent structures, the amount of settlement must be estimated for different types of GWSS methods. Cost trade-offs between cheaper, more flexible, GWSS and underpinning, should be assessed against more rigid but more expensive GWSS which require a minimum of underpinning. The amount of settlement depends upon soil type, size of excavation, construction methods and quality of workmanship. The prediction of the amount of settlement is uncertain and thus experience and observational data are needed as guides to judgment.

Earth pressure envelopes which influence the design of the GWSS, represent the distribution of the cut pressure that is exerted on the sides of the GWSS. They do not necessarily indicate the actual pressure distribution, but rather represent design pressure values that, if used, can be expected to provide a safe and serviceable structure. The pressure envelopes assume that the installation of struts and bracing will proceed as soon as the excavation reaches the level where they are to be placed.

The method of construction is an important factor in determining the actual distribution of pressure on the GWSS. Recommended design pressure envelopes for use in calculating working lateral loads in sand and clay, are found in Peck 
(1984). These distributions are used later to determine the type of GWSS, retaining system, and installation method.

\subsubsection{Common TYpes of GWS8s}

The sheeting part of the outside GWS usually follows the physical dimensions of the tunnel, allowing just enough space to permit construction of the permanent structure. Common types of GWSS, retaining systems, and their installation methods are described below.

Emphasis is placed on GWSS design alternatives of steel sheet piles and soldier piles and lagging. Specific examples of the aforementioned GWSS are used for analyzing Cut-and-Cover tunnelling operations and are used for CMSA implementation as revealed in chapters 3,4 , and 5 .

\section{Sheet Piling}

The function of sheet piling (SP) is to retain soil and ground water. SP may be used to minimize the settlement, though it can never eliminate it. It is used where there is a danger of bottom heave in soft clay soils and sand liquefaction. It is also used to avoid dewatering when there is a risk that lowering the water table may cause subsidence in the surrounding ground. Settlement of this type is not usually a problem if the soil is dense sand or hard/stiff clay strata; however, in loose sand or soft clay, 
substantial settlement can occur if the water table is lowered.

SP is not satisfactory in hard clay, very dense sand or rock bearing strata. The SP is normally designed as continuous sheeting with supports of struts or anchors installed as excavation progresses.

Pile types may be defined by the effect of installation on the soil. Three major types of piles are in common use: displaced, small displaced, and non-displaced piles (Palmer and Tomlinson 1981). The first and second are normally driven, the third is formed by boring or excavation (predrilled pile). SP falls in the second category. It provides lateral resistance against soil pressure but has low bearing capacity.

Sheet Piles can be further classified in terms of their material composition: steel, wood, or concrete (cast in place or pre-cast). Attention will be focused on the first type, steel sheet piles, since its use is predominant in the field of construction.

1.1 steel sheet Piles: structural shapes have been developed for steel sheet piles (SSP) and are in widespread use. They are stronger, more durable, less subject to damage during handling and easier to drive than wood or concrete sheet piles. In addition, they can be made relatively watertight with minimum effort and expertise. 
SSP is reasonably flexible, causing some loss of ground and settlement, which may exceed allowable limits. Another possible source of settlement is compaction of loose granular soil resulting from vibration. From an environmental standpoint, pile driving is a noisy operation, although some relatively quiet methods have been developed.

SSP provides an effective temporary barrier to water except where piles get disturbed or when workmanship is poor. Water tightness of SSP is rarely adequate for permanent tunnel requirements (Megaw and Bartlett 1981). SSP may be left in place after installing the tunnel structure or removed for reuse in other projects.

SSP is available in different forms, sizes, and stiffness. Each form has its own inter-lock system to provide a continuous GWSS.

1.1.2 8teel sheet Pile section Types: Forms of SSP can be classified into the following categories:

1. Z-sections;

2. $\operatorname{arch}$ web (U-section);

3. straight web;

4. special $\mathrm{Y}$ and $\mathrm{T}$ sections.

$\mathrm{U}$ and $\mathrm{Z}$ sections are normally used. Typically, the range of weight of normal sections is about 15 to $60 \mathrm{lbs} / \mathrm{square}$ foot of wall area, with corresponding section modulus of 10 
to 80 cubic inches per foot run of wall. Thickness varies from $1 / 4$ to $7 / 8$ inches, and width varies from 12 to 30 inches. Normal sections are used to resist larger horizontal forces because of their great stiffness, which in turn reduces the bracing required. straight web sheets do not use much space, are comparatively flexible, and hence suitable only for relatively light lateral pressure. They are designed to have a high transverse inter-lock strength in tension, and possess negligible bending strength. They are applicable only for cellular construction.

The notion of efficient sections governs the design process, where efficiency is defined as the ratio of section modulus per foot run of wall to the cost (weight) of piling per square foot of wall. Improved efficiency results from the use of a wider section with some reduction in thickness (Winterkorn and Fang 1975).

Three features give normal sheet pile sections their advantage. These are interlock, shape of the pile cross section, and steel quality. Greater penetration can be obtained with SSP than with concrete or timber SP's which have, comparatively, a much larger cross section. In addition, for a given bending strength, a steel SP is lighter in weight than other materials. Transport and handling into position for driving is simpler and less 
costly. A lighter hammer can be used to achieve the same rate of penetration for SSP than for other materials.

1.1.3 ssP Design: The main forces on SSP are from soil pressure and the support system of struts or anchorages. Several methods can be used for the computation of lateral pressure distribution. Some of the better known ones are attributed to Tschzaboterioff (1951), Terzaghi and Peck (1967) and Peck (1969) for different types of soil.

The design process for strutted excavation is now regarded as completely empirical for estimating the bracing loads, and subject to construction procedures such as welding, and dewatering methods (Ratay 1984).

A general design procedure for steel sheet pile is as follows (Winterkorn 1981):

1. compute bending moments from the earth pressure distribution and strut reaction on the wall;

2. compute the section modulus for the sheets and consult the allowable stress; and

3. from standard AISC ${ }^{1}$ for steel sheet piles, select a corresponding section (grade, strength, size, and other properties).

The same design methods are applicable to the design of soldier piles in the Soldier Piles and Lagging alternative. The above procedure represents the conventional design approach for a single wall braced structure of sheet piles.

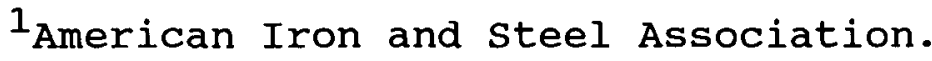


See Bowles (1977) for more modern design procedures based on the finite element method. Appendix A presents the design computation method adapted for the construction Methods Selection Assessment prototype (CMSA) described in chapters 4 and 5 .

1.1.4 Construction Resources for ssp installation (Pile drivers): $\quad$ Construction resources applied for SSP installation include cranes (fixed and/or mobile), pile driver (vibratory and/or hammers), and crews required to drive ssp to their refusal depth. Among those resources, pile drivers are treated as variables and the rest are treated as fixed in the actual project context for CMSA development in chapter 4 .

The function of a pile hammer is to furnish the energy to drive a pile. Pile drivers are designated by type and size. Hammer types are commonly classified as:

1. drop hammer;

2. single acting hammer (steam or air or diesel);

3. double acting hammer (steam or air or diesel);

4. differential acting hammer; and

5. hydraulic hammer.

When noise is a problem, the following can be used:

1. TW pile master; and

2. vibratory pile driver. 
operating principles, descriptive data, and relative advantages and disadvantages of each of the above pile drivers can be found in Jones (1963) and Peurifoy (1970). It is important to note that the driving capability of hammers is generally expressed in its theoretical foot-pound (ft-lb) of energy delivered per blow.

selecting a Pile Hammer: Factors that influence hammer selection include the size and type of piles, the number of piles, the soil condition, the location of the project, topography of the site, and type of rig available. Given the different sheet pile and pile driver types available, and the range of soil types and job conditions encountered, selecting the best pile type and hammer is a non-trivial task (Jones 1963).

The contractor is usually concerned with selecting a hammer that will drive piles for his project at the lowest cost. Manufacturers' recommendations are seldom reliable. There are some rules of thumb that help in selecting the size of the hammer. As an example, the maximum ratio of pile weight to ram weight should be four to one as suggested by Vulcan Workers, Inc. (Jones 1963).

A more rational selection process is the one suggested by Peurifoy (1970). He presented, in tabular form, data for selecting pile hammers for driving different types of piles in a variety of soil conditions. Data from this reference 
were used in the CMSA prototype developed as part of this thesis for the two soil types previously described.

\section{Soldier Pile and Lagging}

steel soldier piles in the form of $H$ steel sections are placed prior to the start of excavation by driving or drilling at typically 8 to 10 foot centers. Horizontal lagging is placed between the piles as the excavation progresses. The minimum thickness of lagging should be $1 / 24$ of the span width (Ratay 1984). A variant is where a length of wall is concreted in a slurry filled trench excavated between the King piles (concrete placed around the $H$ steel sections after placing the sections in a pre-drilled hole). The piles can be designed to carry a temporary road deck, under which construction can proceed. Lagging can be eliminated if the soil is highly cohesive and capable of arching. It is inappropriate to use this method in perfectly cohesionless soil in which sheet piles must be used (Schroeder 1980).

Possible settlement of adjacent structures must be considered, and headroom for setting the piles has to be ensured. 


\section{Insitu Reinforcement Techniques -- Soil Nailing}

Recently, new in-situ earth reinforcement and lateral support systems for deep excavations have been introduced. Unlike conventional systems that serve to retain soil behind a positive support system, the use of these alternative systems is based on the concept of soil reinforcement. That is, native soil adjacent to the excavation is strengthened so that it can stand unsupported at depths which would normally require the installation of conventional GWSS (e.g. Soldier Piles, sheet Piling).

Three main categories of insitu reinforcement exist: soil nailing, reticulated micro-piling, and dowelling. In soil nailing, the reinforcement is installed horizontally immediately after excavation so that it improves the shearing resistance of soil by acting in tension. Soil nailing works by reinforcing the ground in situ with relatively small, fully-bonded inclusions, usually steel bars (Bruce 1987). They are introduced to the soil mass, the face of which is locally stabilized by sprayed concrete, and act to provide a zone of reinforced ground.

Although the soil nailing system is intended to serve as temporary support only, it has been shown that it has a great deal of long term stability (Shen 1981b).

Reticulated micro-piles are steeply inclined into soil at various angles both perpendicular and parallel to the face. 
The overall aim is similar to soil nailing: to provide a stable block of reinforced soil acting like a retaining structure. The reinforcement acts to resist bending and shearing forces.

Soil dowelling is applied to reduce or slow down slope movements on a well defined shear surface. The reinforcement provides resistance to shear forces. 'The pile diameter for soil dowelling is generally far greater than that for soil nailing.

\section{Concrete Bored Piles}

These are large diameter bored piles at close centers that can be used as... a wall. They might be contiguous or may overlap to form what is called secant Piles. With good workmanship, substantial water tightness is possible, particularly with secant piles.

\section{Slurry Trench Wall}

The slurry trench wall (STW) technique, also called Diaphragm Concrete walls, is relatively new. STW can be applied to construct two types of walls. These are concrete Diaphragm wall and Soldier Pile Tremie Concrete Wall.

5.1 Concrete Diaphragm Wal1: Constructed as a normal cast in place wall with supports, a concrete diaphragm wall provides a watertight barrier which can be installed with a minimum of back slope subsidence. The wall becomes part of the permanent structure. Because this wall is impervious, 
dewatering of granular soils is often not required. Diaphragm walls are considered to be semi-rigid and walls of shallow depth in moderate ground conditions are sometimes left unsupported. In a deep excavation, support is required to restrict lateral deflections. Skin friction along the wall tends to reduce settlement of adjacent soils below an amount which is common for soldier piles or steel sheet piles. It is reported that vertical settlement appears to be in the order of $0.25 \%$ of excavation depth for diaphragm walls versus generally less than $0.40 \%$ for other support systems (Ratay 1984).

5.2 Soldier Pile Tremie concrete wall (SPTC): This is a modification of the standard STW techniques to increase lateral resistance. It consists of a tremie concrete wall in which steel soldier piles are embedded for reinforcement. For deep excavations in soft ground, these walls may help alleviate potential subsidence problems, but they still require some retaining system.

\subsection{Excavation Operations}

The excavation activity can be divided into a primary excavation of most of the tunnel space bulk, and a secondary one that is prior to GWSS installation. Examples of the latter include pre-drilling and/or excavation for secant piles installation, and slurry trench wall. Excavation can 
proceed simultaneously both vertically and horizontally. The pace of excavation is a function of soil cohesion, tunnel dimensions, the GWSS, and the muck removal system selected.

within the methods definition in this thesis, the excavation activity represents a tactical construction operation that could be separable and distinct yet integral to the GWSS type and alternative, and can be described in terms of its resources (the reader is referred to the interviews in Appendix C). Section 3.8, a specific Cut-andCover tunnelling example, examines and structures pertinent excavation roles in balancing a construction cycle.

Excavation, in Cut-and-Cover tunnelling, is substantially different from underground tunnelling in terms of operations, construction methods and scheduling. For a given tunnel, it can proceed in two directions: horizontally, parallel to the tunnel, and vertically, toward the bottom of the tunnel. The pace of excavation in either direction is subject to soil stratification, tunnel dimensions, retaining system, compatibility of muck removal system, and so forth.

The excavation progress, interwoven with other activities, eventually yields a construction cycle. Excavation cycle optimization has a substantial impact on total productivity and production rates. 
For shallow cuts, the excavation is usually made from the surface, using a backhoe, and dumping the muck directly into trucks or into a stock pile along the side of the excavation. For deep cuts, the excavation may be carried out using trucks with haul ramps, or clamshell hoists, or by using cranes to lift the excavated material from the bottom cut into the stock pile or waiting trucks.

A wide range of equipment may be used, such as backhoes, front end loaders, bulldozers, trucks, draglines, scrapers, clamshells and cranes. Each type of equipment comes in different capacities and is preferred depending on job conditions.

Some variables that affect the excavator productivity are (Caterpillar 1982):

1. Soil type and condition

1. conduit type and size;

2. number of cross lines;

3. trench dimension;

4. spoil pile dimensions;

5. truck loading requirements;

6. operating ability; and

7. job management.

\section{Machine related factors}

1. proper attachment for the job;

2. size of digging and spoiling envelope; 
3. payload capability (bucket size);

4. cycle time (breakout force and hydraulic speed); and

5. Iifting capability.

After describing various Cut-and-Cover tunnelling alternatives, one needs to consider the major factors that influence alternative choices. These are discussed next.

\subsection{Factors Affecting Methods Selection and Design}

Cut-and-Cover tunnelling construction methods embrace a wide range of alternatives. Influencing factors may interact and/or override others under different project conditions. It is not always immediately obvious what is the best solution, due to the complexity of the interaction among operations, and given the project context variables, such as ground conditions and access to the work area. It is useful to explore some of these factors in more detail to demonstrate the diversity of problems that have to be treated, the range of methods, etc. Where appropriate, reference is made to simplifying assumptions adopted for illustration purposes in the thesis.

\section{Ground conditions}

Among various influencing factors, ground conditions dominate the choice of construction methods. Ground conditions do not always fall into convenient and clearly defined groups. Soil has relatively low strength and high 
deformability. These adverse mechanical characteristics have a direct influence on excavation, stability, type of permanent structure, and compaction methods. Ground conditions generally can be divided into five broad groups:

1. Cohesive soil;

2. Noncohesive soil;

3. Soft rock;

4. Hard rock; and

5. Organic soil.

To reduce the size and dimensionality of the problem at hand, only a small subset of soil types will be considered in this study. Mutually exclusive soil types included in this study are: loose sand, and dense sand; and soft clay, and hard or stiff clay. The maximum number of layers is assumed to be two. These limitations were set so that the number of soil strata scenarios (e.g. loose sand on top of hard clay is one out of 12 perturbations of soil layers order) will be a relatively small size which simplifies the choice of a method.

2. Ground water conditions

The presence of water, its level, and its chemical composition may dictate compatible control methods that minimize ground subsidence. This could be done by lowering the ground water table, by grouting, freezing, compressed air, or a combination of the foregoing. Further, water 
exerts pore pressure onto the sides of the excavation in addition to the soil pressure which creates extra pressure on the ground wall support system.

\section{Size of the proposed excavation}

The physical dimensions of the trench have a substantial impact on almost every method used during construction. The dimensions, along with geotechnical conditions, dictate the proper equipment for the excavation and material handing strategies. For instance, a shallow depth may suggest the use of a backhoe, a front shovel, or a scraper, while deeper trenches require the use of a clamshell or a crane with a loader or a dragline.

\section{Working space access}

Some cut \& Cover techniques may require more working space and access than other competing alternatives. This may be of great importance especially where allowable work space is small and/or congested.

\section{Diversion of services}

Removal of underground utilities or diversion of buried services before excavation is the ideal for cut-and-Cover tunnelling. However, this is rarely possible for all services. Temporary diversion, or the cutting-off of some services may be unacceptable, especially in urban areas. Those which need to cross the trench must be supported, 
protected, and maintained during the entire construction process. Such situations may result in undesirable delays.

\section{Environmental requirements}

Construction operations may result in traffic re-routing, closing segments of roads to pedestrians, destabilizing of adjacent structures, and producing hazards (e.g. noise, water contamination). Environmental considerations may eliminate potential methods, restrict daily working time, impose a geotechnical monitoring program, and demand more safety procedures.

Next, a traditional cut-and-Cover tunnelling project will be described to give a flavor to the activities breakdown, planning techniques used, constraints imposed on operations, GWSS installation and excavation interaction, all within a specific project context.

\subsection{Cut and Cover Tunnelling Project Example}

\subsubsection{Background}

The objective of this example is multifold: highlight some Cut-and-Cover tunnelling characteristics and spatial constraints; and show how decisions (production rate, resource selection, etc) pertaining to the excavation construction cycle, and GWSS retaining system design elements (spacing of retaining system), are interwoven with excavation. This example serves as a reference for 
subsequent variations of the Cut-and-cover tunnelling projects throughout the thesis.

Further, a Cut-and-cover project in downtown seattle (Turnham 1988), is adapted to exploit, characterize, and implement the ideas and solutions for methods selection. Table 3.2 displays information extracted from the project with some modification.

\section{Location:}

Type of Project:

Tunnel Dimensions:

Type of GWSS:

Ground Condition:

Progress Rate:

Ground Work Control:

Ground Treatment:

Underpinning and Utilities:

Design Requirements:

Project Duration:

Selection of Methods:

Type of Retaining System:
Downtown Seattle

Metro Tunnel

38 feet wide, 60 feet deep, and 800 feet long.

Soldier Piling and Lagging

Soft Ground (tough dry clay)

30 feet per day (for excavation and lagging)

Pumping

Not Available

None

Excavation does not exceed 10 feet of depth without lagging and bracing.

May 1 to November 1, 1987

Not Available

Struts (and/or Tie-backs)

Table 3.2 Seattle Project General Information [Adapted from Turnham 1988]

Figure 3.2 is an illustration of the aforementioned reported project during construction. 


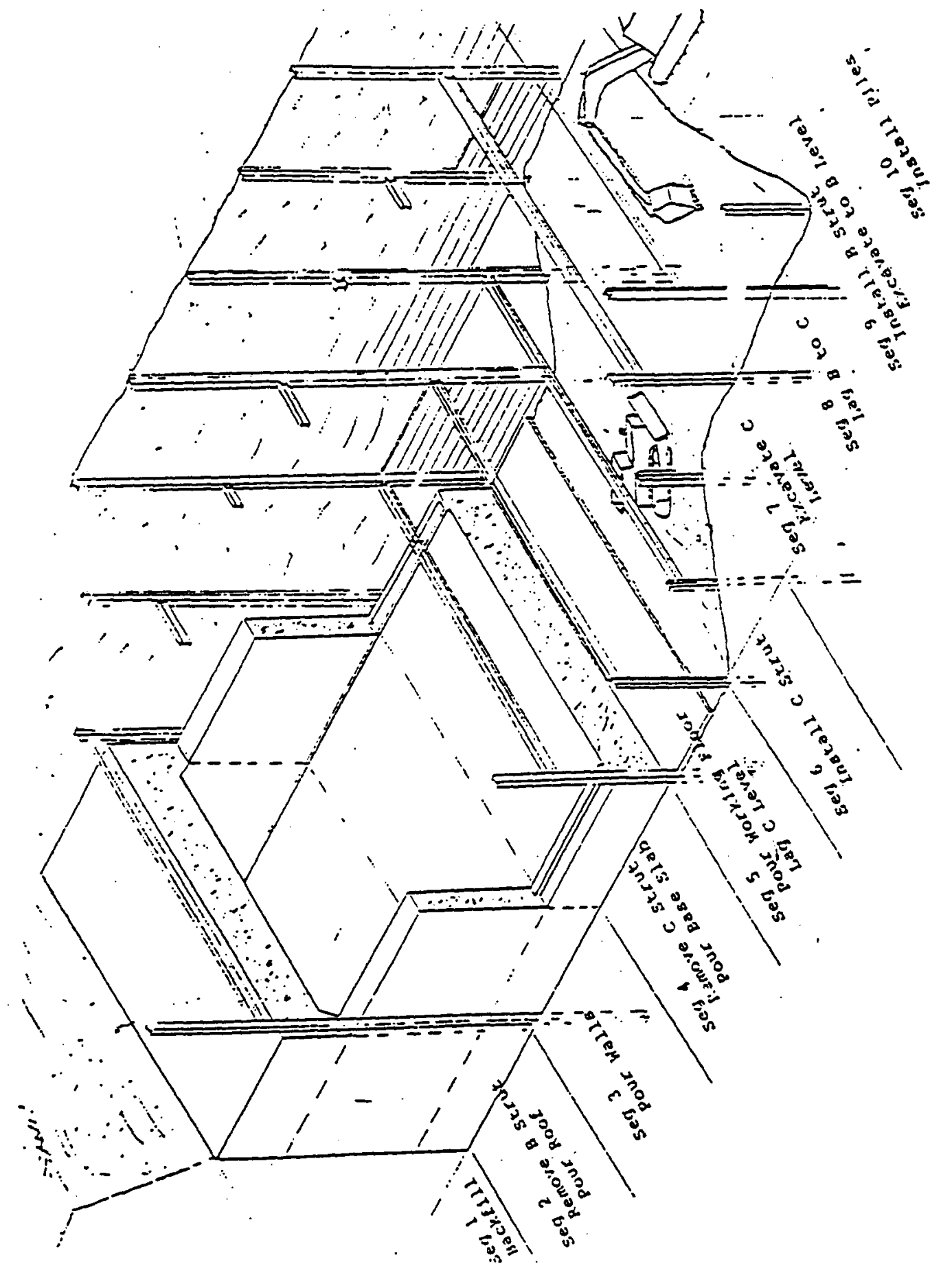

Figure 3.2 Cut and Cover Tunnel project Isometric view for Boldier Piles and Lagging with struts for seattle Project [Turnham 1988] 


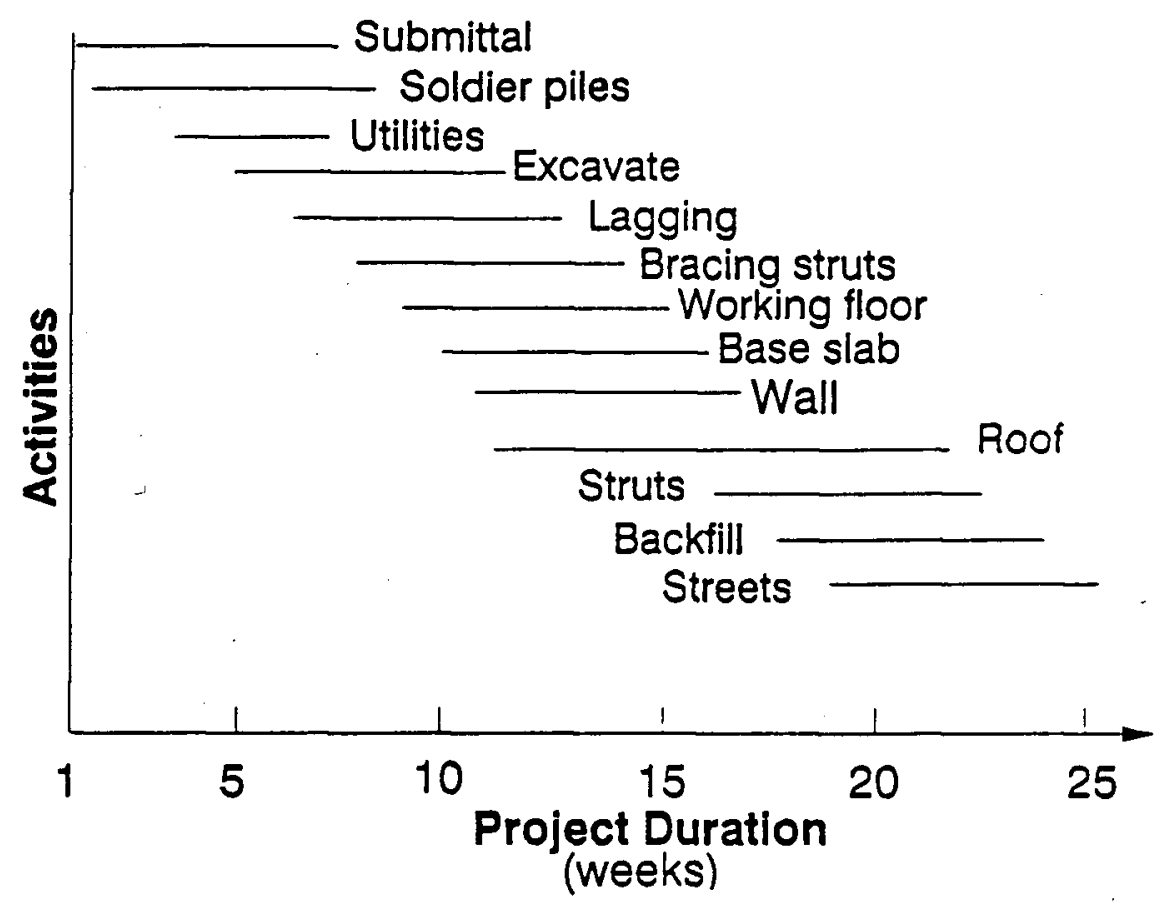

(a) Projeat Barchart

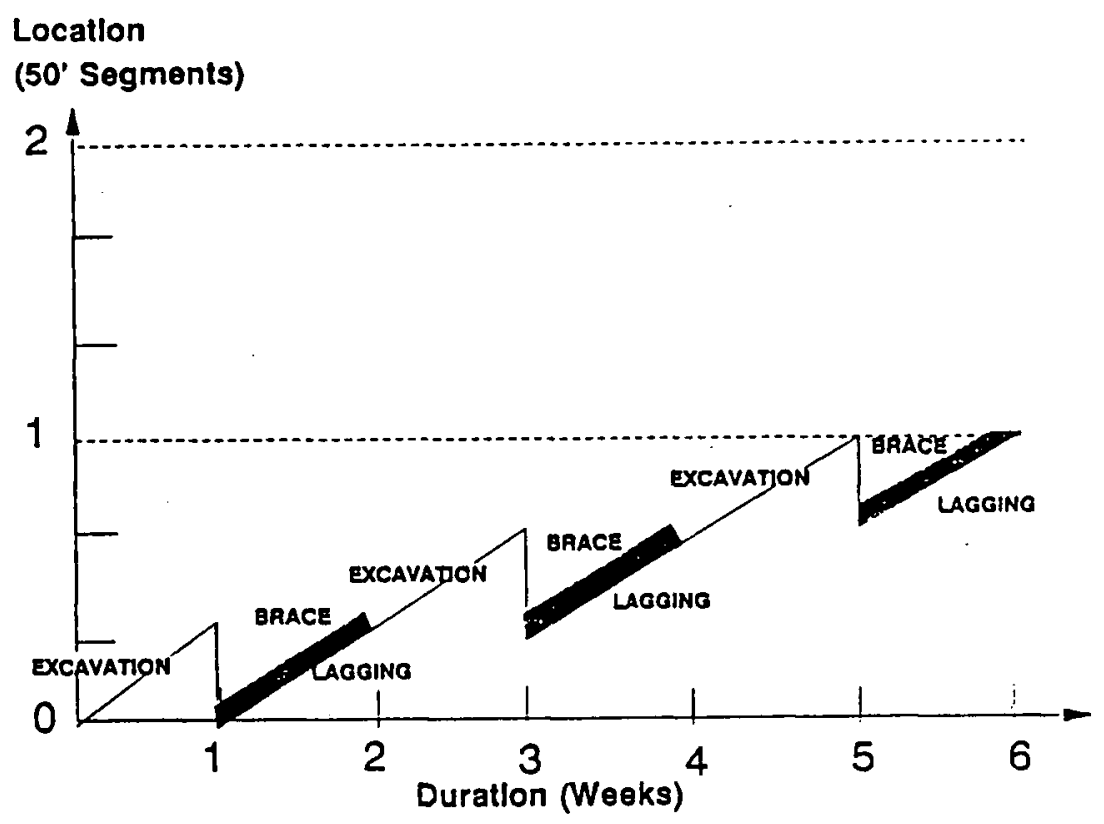

(b) Project Time-8pace Diagram

Figure 3.3 Barchart and Time-8pace Diagram for the Beattle Project

[Erom Turnham 1988] 
The site sketch of figure 3.2 presents, from right to left, the following flow of work: primary excavation at the top done by a backhoe; secondary excavation at the bottom of the trench done by a bulldozer; pre-installation of soldier piles as GWSS; installation of struts as retaining system; construction of permanent concrete tunnel floor; and placement of sides and roof; and, finally, backfilling.

For this cut-and-Cover example, the bar chart and space time diagram for major activities, shown in figure 3.3 $(a, b)$, serve as a planning tool.

\subsubsection{Lagging and Excavation Construction Cycle}

A construction cycle can be defined as a sequenced repetition of construction operations that constructs a prespecified modular unit of a facility. For instance, a floor in a high-rise building or tunnel excavation unit step, or a house unit in a residential housing project. A construction cycle is mostly applicable to linear projects. For this example, the project engineer first creates barchart and space-time diagrams to show major activities, their logical sequence, and their inter-relationship. These planning tools set the pace of production for construction operations, and sets the total duration for the project activities. The contract project target duration of six months sets the pace for the required production rate for each successive activity. 
The excavation activity will be the lead one that triggers lagging and other following activities. Lagging, on the other hand, could delay excavation if its installation pace in the vertical direction is slowed down to less than the excavation production rate. Therefore, in order to optimize the cycle production, the rate of lagging production should equal, or be slightly less than the excavation rate.

one can picture, physically and geometrically by dividing the tunnel into segments, the sequence and the pace of excavation and lagging per construction cycle. First, excavation proceeds horizontally at the top layer, and after finishing the second segment, lagging starts at the first segment and at the same rate of the former operation. Thus time and space lags are specified. Figures $3.4 \mathrm{a}, \mathrm{b}, \mathrm{c}$, and $\mathrm{d}$ show the routine successive progress of excavation and lagging for the soldier piles and lagging alternative.

To summarize the aforementioned description, the identified major construction cycle decision variables are:

1. Segment dimensions;

2. Depth of excavation:

3. Direction mode for Excavation;

4. Time lag between activities; and

5. Space lag between crews (in number of segments). 


\subsubsection{Constraints for Construction Operations}

Central to the construction cycle for lagging installation and excavation operations, are two categories of constraints.

\section{Engineering Design Constraints}

First, the type of GWSS (Soldier Piles and Lagging) dictates a top-down excavation strategy. Design specifications prohibit excavations to proceed more than 10 feet below lagging nor deeper than 12 feet below bracing. Therefore, excavation and lagging were constrained to follow a leapfrog sequencing to reach the required 60 foot depth. Figure 3.4 shows the time-space diagram for these two activities, where the rate of production for both excavation and lagging, and the time lag between them, is suited to complete one segment of 50 feet of permanent tunnel structure.

\section{Geometric Constraints}

The tunnel structure forces equipment and formwork (shoring) to move in the direction of the tunnel. Typically, for wide and deep tunnels, excavation proceeds in angled slides (slopes) rather than vertical slides (shallow tunnels) to allow excavating and hauling equipment easy excess. In addition, the slope stabilizes itself with no support.

The size of the tunnel and project location allows several combinations of methods. For example, for the method attribute of construction resource, a backhoe at the 
top half of the tunnel is excavating and hauling, whereas a bulldozer excavates the bottom-half and pushes it to the upper half within the reach of the backhoe, as shown in figure 3.2. An alternative for the previous combination may include a front end loader for excavation and a crane for hauling the muck into trucks. The dimensions of the tunnel may allow smaller equipment to work in parallel, or alternatively, one large machine.

Careful selection of methods for major operations requires defining the tasks that a method performs and allocating resources for these methods. Some resources may perform multiple tasks, such as a backhoe and a crane, whereas others perform specific tasks, such as front end loader (hauling muck). Therefore, geometric constraints allow only some scenarios of construction methods combinations.

our intention here was to hint at the low level construction process variables. Chapter 4 shows how these decision variables are incorporated into a method frame. 


\section{A KBES Framework for Methods Selection and Design}

\subsection{Introduction}

The purpose of this chapter is to structure the methods selection problem by describing the problem, proposing a KBES framework approach, and prescribing the attributes for a control strategy shell. This chapter builds, step-bystep, a vision of a systematic knowledge based approach for the methods selection problem.

Set out in section 4.2 is a KBES framework for this problem. This framework reflects the hierarchical structure of the decision making process. A method is described by its attributes which include design element, construction strategy, construction resources, and construction method process. A conceptual KBES that integrates procedural, factual, and judgmental knowledge approaches for methods selection is then described. This is followed by a description of system features and a pseudo rule-based control strategy. The goal of the system is to eliminate undesirable alternatives, rank the remaining ones and suggest values for the attributes of feasible methods. An example for cut-and-Cover tunnelling is presented to demonstrate how the KBES framework is intended to work. 
Steel sheet piling is selected as the example context for this implementation. Described in section 4.3 .2 are the decisions variables and project context variables treated for this example.

In section 4.3 .4 , a conceptual control strategy is described. The control strategy treats methods selection in a two step process: preliminary feasibility, which screens out undesirable alternatives; and detailed feasibility, which specifies the remaining alternatives.

Key criteria which make a method accepted or rejected at the two levels in the control strategy are identified, and include: design, risk, resource compatibility, performance measures, and regulatory considerations.

At the preliminary feasibility level, high level rules based on empirical knowledge are used to reduce alternatives. Detailed feasibility analysis involves a multi-stage process. It attempts to synthesize design attributes of the methods that pass through preliminary feasibility.

Once a detailed synthesis is successfully completed for a method, it is evaluated and ranked against other feasible alternatives. Evaluation criteria based on costs are used to rank alternatives at both levels. At the preliminary level, alternatives will be ranked according to their 
preliminary unit costs. At the detailed level, costs include an assessment of risk in terms of money.

The risk assessment framework adopted is elaborated in section 4.4. Risks have been classified into normal (acceptable) and catastrophic (unacceptable) risks. The former includes risk categories of equipment, material, productivity, subsurface subsidence, seasonal, and other losses. A decision tree incorporating three states of nature for encountered subsurface conditions, is used to treat risk. These are: encountered conditions are more favorable than expected, as expected, and worse than expected. From the latter, three further states of nature are treated : no damage, element damage, and system damage. If the probability of system damage exceeds a threshold value, catastrophic damage is assumed to occur and the method alternative is rejected, otherwise, it will be accepted. The aforementioned risk categories are applied at the decision tree terminals as possible outcomes. 


\subsection{A KBES framework for Method Selection}

\subsubsection{General}

In this section, a general KBES framework for method selection is presented. The method definition introduced in section 2.2.1 is further refined to provide this framework. For discussion purposes, we treat time as the main criterion at this stage, while cost, quality, safety, and others, are treated as secondary goals that could be violated within a range. A pseudo rule-based control strategy is used to illustrate operations of the framework.

\subsubsection{Methods Selection Defined}

Based on the discussion in section 2.2.1, the term "construction method" is used in a wholistic sense. That is, it embraces design, strategy, resource and process considerations. Specifically, a construction method is described in terms of a conceptual frame. Attributes of this frame are structured in a hierarchical fashion. They. include design concept for temporary facilities, construction strategy, resource requirements and construction process. Not all levels are meaningful for every methods selection problem.

To illustrate the use of the definition, consider figure 4.1 and table 4.1. 


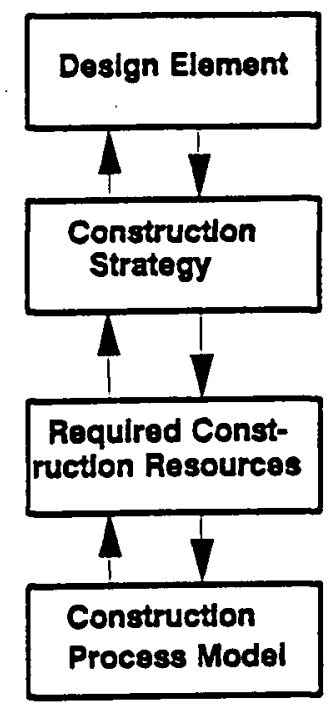

(a)

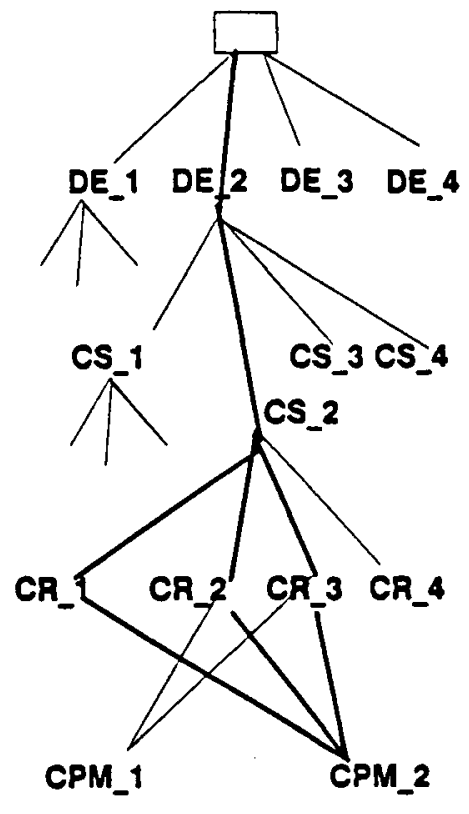

(b)

Figure 4.1 Hierarchy of Construction Method Frame attributes

Figure 4.1 (a) depicts a hierarchy of the method attributes, while figure 4.1 (b) exhibits various attribute alternatives at each level, and the bold line represents the most preferred alternative at each level, which are taken together to define a construction method.

Table 4.1, corresponds to figure 4.1. It reflects the kinds of alternatives available and decisions to be made at each level for the Cut-and-Cover tunnel problem. Design alternatives refer to the GWS temporary structure as decision variables, whereas the permanent tunnel structure is treated as fixed. Each GWS alternative has its own design and construction procedures as briefly described in chapter 3. Lower level decisions pertinent to this level 
imply specifying structural members for the GWSS and the retaining system, including design criteria selection (moments, deflection, etc.) and type of materials employed. For instance, specifying a GWSS of steel sheet piles includes their members sections selection and sizing based on moment criterion, where steel grade, section modulus, moment of interia, and other properties characterize a member.

At the construction strategy phase, two levels are identified. The first one is a high level decision, whether an upside-top (Milano Method) should be utilized as opposed to the traditional top down, which dictates the sequence of construction operations and scheduling.

Lower level decisions include tactical (operational) decisions such as selecting operations variable values, and retaining system. For example, the operations variables of a GWSS installation for pile driving include piles driven in waves, in singles or in doubles. For the latter, retaining systems include two choices: struts and tie-backs, which provide obstruction free access for construction operations and thus shorten the construction duration. 


\section{LEVEL}

\section{Design Alternative For Temporary Facilities}

Ground Wall Support System (GWSS)

Temporary/Permanent GWSS

\section{Construction Strategy}

High Level Strategy

Pile Driving Patterns

Retaining System

\section{Construction Resource Requirements}

GWSS Installation

Ret. System Installation

\section{Construction Processes Model}

Cut-and-Cover Tun. Process

GWSS Installation \&

Excavation Are Separable

GWSS installation \&

Excavation Are Intertwined

\section{ALTERNATIVES}

Soldier Piles \& Lagging

Steel Sheet Piling

Slurry Trench Wall

Soil Nailing

Shotcrete

Secant Piling

Upside-Top;

Top-Down

Waves, Doubles, Singles

Wales and Struts,

Tiebacks

Pile Drivers

Drilled Piles

Lagging Installation

Crew_B_20

.

Network Models

Simulation, CPM, . .

Process (GWSS) + Process (EXC)

+ Process (..) +..

Process $\{$ GWSS + EXC $\}$

\section{Table 4.1 Methods selection space for GWS}

The third attribute, construction resource requirement, takes in a pool of available resources to construct the GWSS facility. For instance, if steel sheet piles was considered feasible as a design alternative, and a top-down construction strategy is selected, then the availability of the materials and resources for the GWS and the retaining 
system -- sheet piles, struts/tie-backs, wales, pile drivers and crews are critical to the acceptance of the GWSS alternative.

The construction process model embraces criteria for measuring the performances of alternatives at several levels: project, activity, and operation levels. For instance, the progress rate of the two prominent activities of GWSS installation and excavation may set the project pace. Those activities may be separate -- e.g. Process (GWSS) + Process (EXCAVATION) -- that is a process per activity as shown in Table 4.1 , in the case of the steel sheet piles alternative; or activities may be intertwined-e.g. Process \{GWSS + EXCAVATION\} -- that is a process for more than one activity, as for the soil nailing alternative. Activities broken down into smaller tasks with allocated resources are emulated via a predictive/analytical process that results in performance measure values. Three things ought to be verified for prescribing a process: the performance measures required (time, cost, safety, quality) and the process to be applied (simulation for excavation, dynamic formulas for pile driving), and construction resources (pile drivers, cranes), given that existing resources are compatible (pile driver size dictates crane size). For instance, at the project level, the critical path method could be used as a predictive and analytical 
tool for project total duration. For lower level operations, such as the pile driving activity, dynamic hammer formulas are used to predict pile driving rate for a given soil/hammer/pile scenario, while "automated" rule based knowledge is used for diagnosis.

\subsubsection{Methods shell}

The basic structure corresponds to a methods selection shell in which we use expert systems to control the selection process as well as to encode knowledge pertaining to various project contexts displayed in figure 4.2. Relevant functions, task descriptions, data and knowledge bases, and analysis tools, are shown for each component, as appropriate.

This notion of a shell is reflected in the various applications modules such as Cut-and-Cover tunneling, Highrise structural work, Pier construction for bridges, etc. 


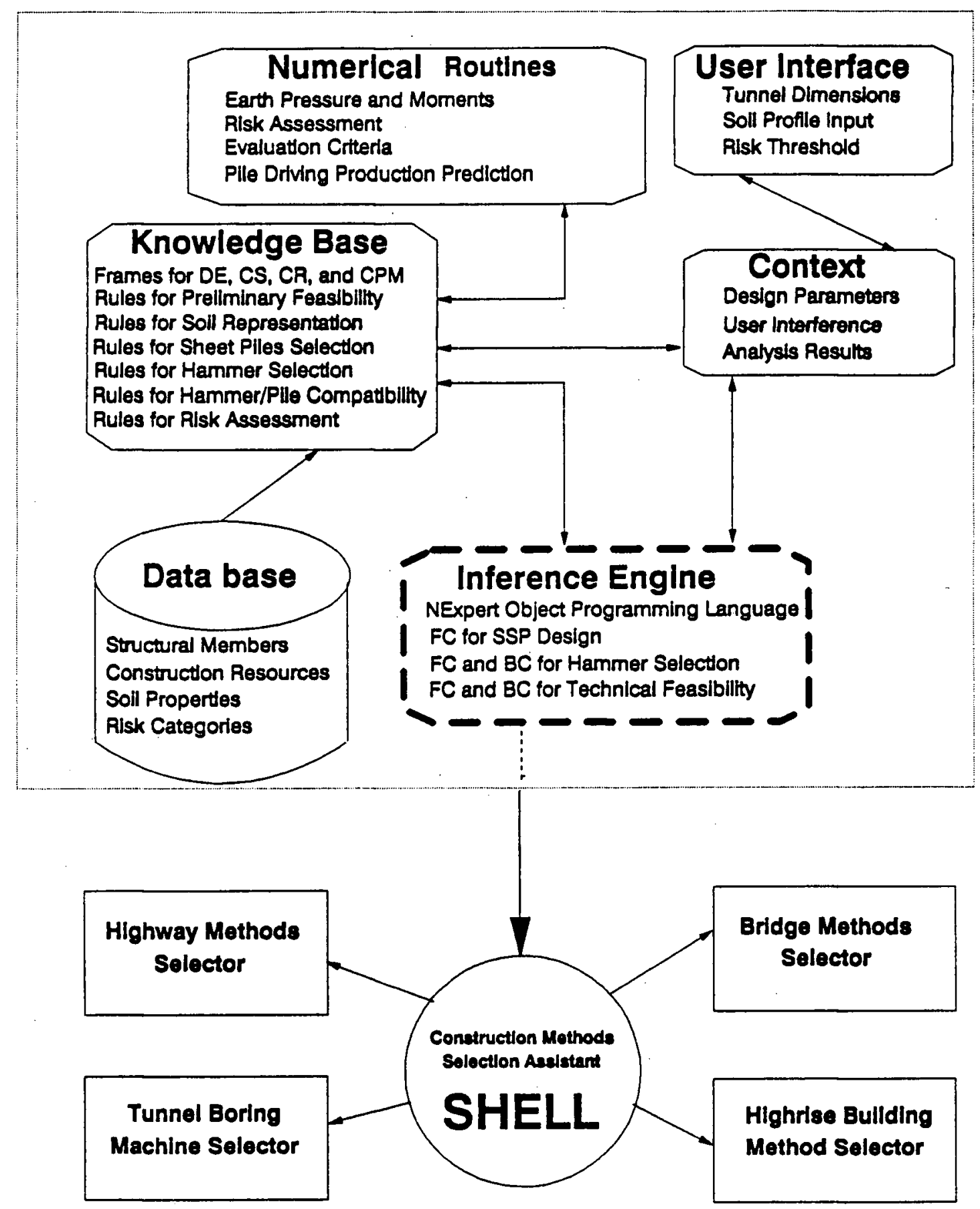

Figure 4.2 Construction Methods selection shell 
Major components of this shell include:

1. User interface to facilitate user input, intervention, and system shell interrogation. For instance, the user may override the system ranking of preliminary feasible GWSS alternatives and answer queries with uncertainty;

2. Knowledge base to contain procedural and declarative knowledge required to specify and marry method attributes;

3. Context reflects the current problem environment during a session. Derived context variables of lateral pressure, moments, progress rate, method attributes evaluation, and so forth, are retained in the context file structure;

4. Inference engine performs the methods selection solution and controls the system session, screening and ranking methods alternatives, instructing the knowledge base to find attribute parameter values, interacting with the user, and so forth;

5. Numeric routines include procedural and analysis knowledge that is encoded in traditional programming languages, e.g., design procedures based on strength criterion, predictive modules based on simulation, etc; and

6. Data base classifies and stores methods in terms of their attributes, structural members, construction resources, soil properties, etc., that are required for method synthesis and accessed by the knowledge base.

In section 4.2 , emphasis is placed on items 2 to 4 while section 4.3 and section 5.3 focus on items 2 to 6 . 


\subsection{4 sketch of system Features and operation}

A two phase system is conceived for ranking and synthesizing methods alternatives: identification and elimination of preliminary feasible alternatives (phase 1), and detailed specification for the feasible methods (phase 2). Figure 4.3. shows a sketch of the components of two phases. The objective of phase 1 is to identify and eliminate the number of preliminary feasible alternatives, thus, reducing them to a few candidates. Phase 2 further carries out a feasibility analysis and methods specification at a detailed level. In relation to figure 4.2 , the following discussion basically covers the role of the inference engine (control strategy), and describes the specific knowledge for the methods selection problem solution.

Phase 1 considers all alternatives options for methods under a scenario of project context and goals. Preliminary project context information may include site layout and access, soil profile and conditions, water table conditions, site location, etc. Project goal includes duration, cost, safety, etc.

Preliminary screening follows after possible methods have been identified. Some will be eliminated through considerations of one or more criteria (e.g. cost, time, dimensionless number). Section, 4.3 .3 presents the kind of knowledge central to evaluating preliminary feasibility . 
Assignment of values to the attributes, which define a construction method, takes place at the detailed level, i.e. phase 2, which constitutes the body of the thesis. The process envisaged, as shown in figure 4.3 , is further elaborated on in figure 4.4 .

Phase 2 consists of three main parts: low-level method specification of the preliminary feasible candidates including specifying method attributes, process modelling, and method analysis.

Also illustrated in figure 4.4 are system functions and example specifications for the cut-and-cover tunneling problem example.

What follows is a step by step description of the application of phase 2 of the model shown in figure 4.3. In giving this description, important issues to be resolved are identified.

\section{Step 1}

The process starts with the user inputting information relevant to the project context. This information can be structured under several headings, as illustrated in Table 4. 2 . 


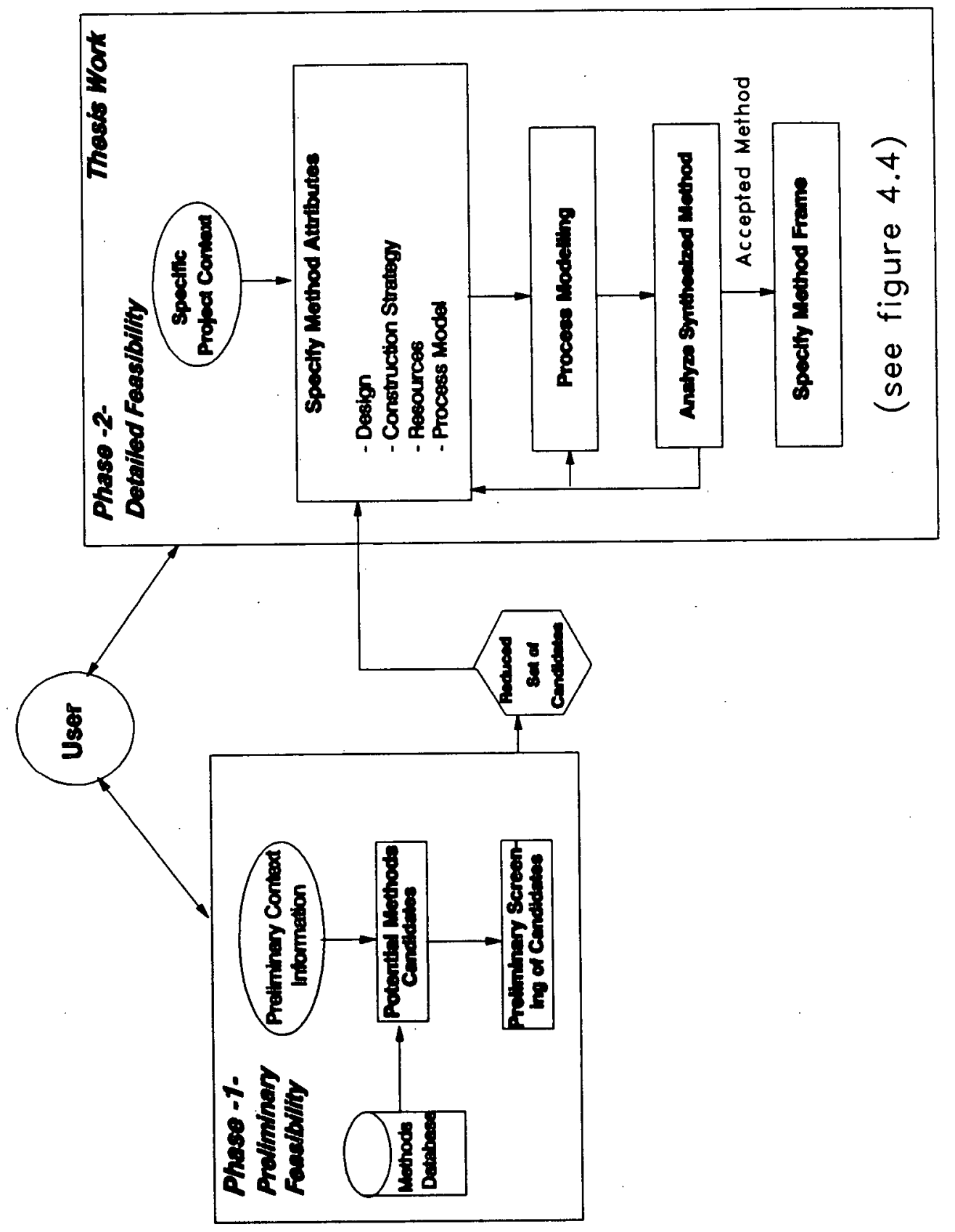

Figure 4.3 Construction Methods selection system Process 


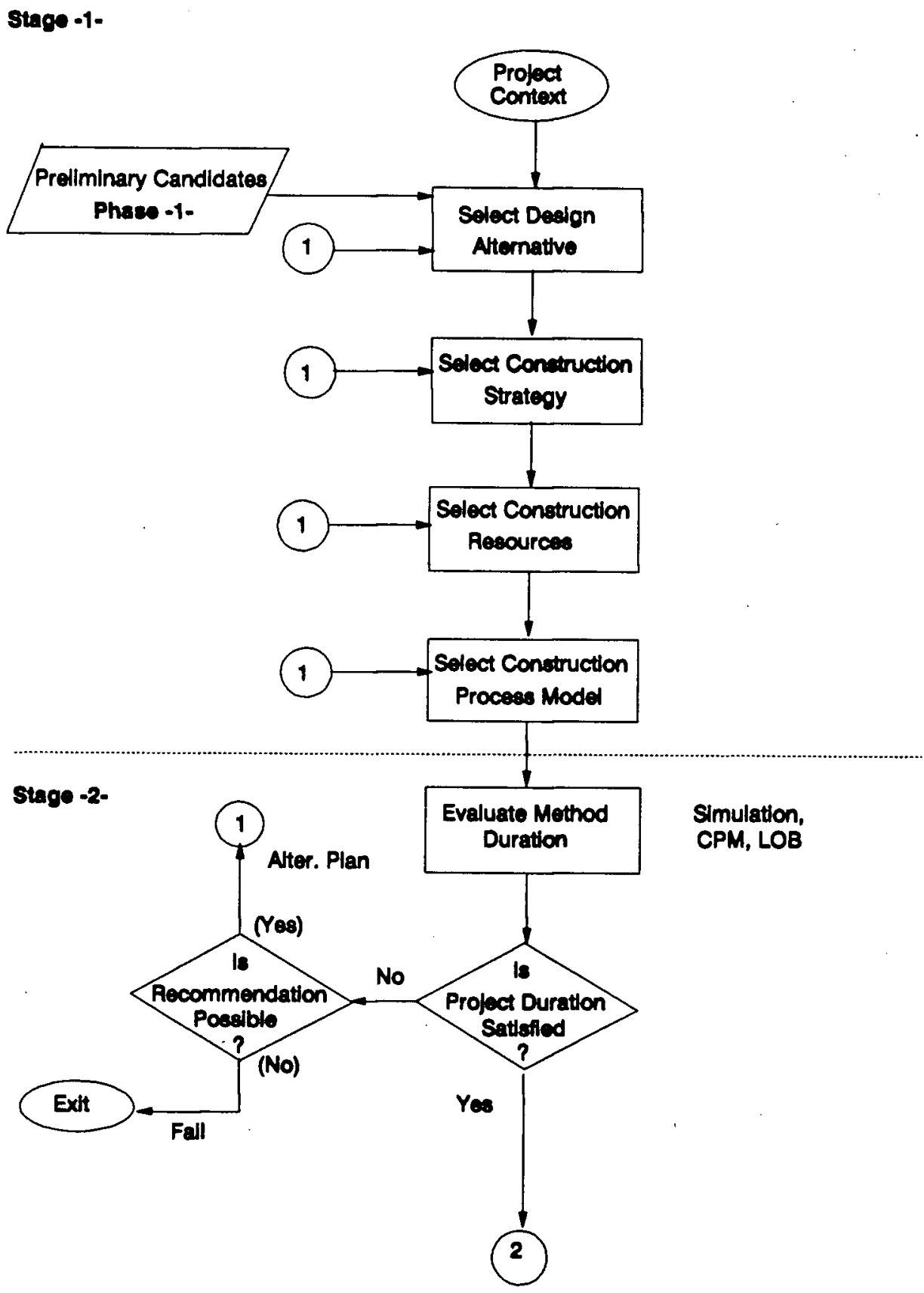

Figure 4.4 Detalled Feasibility (Phase 2) 


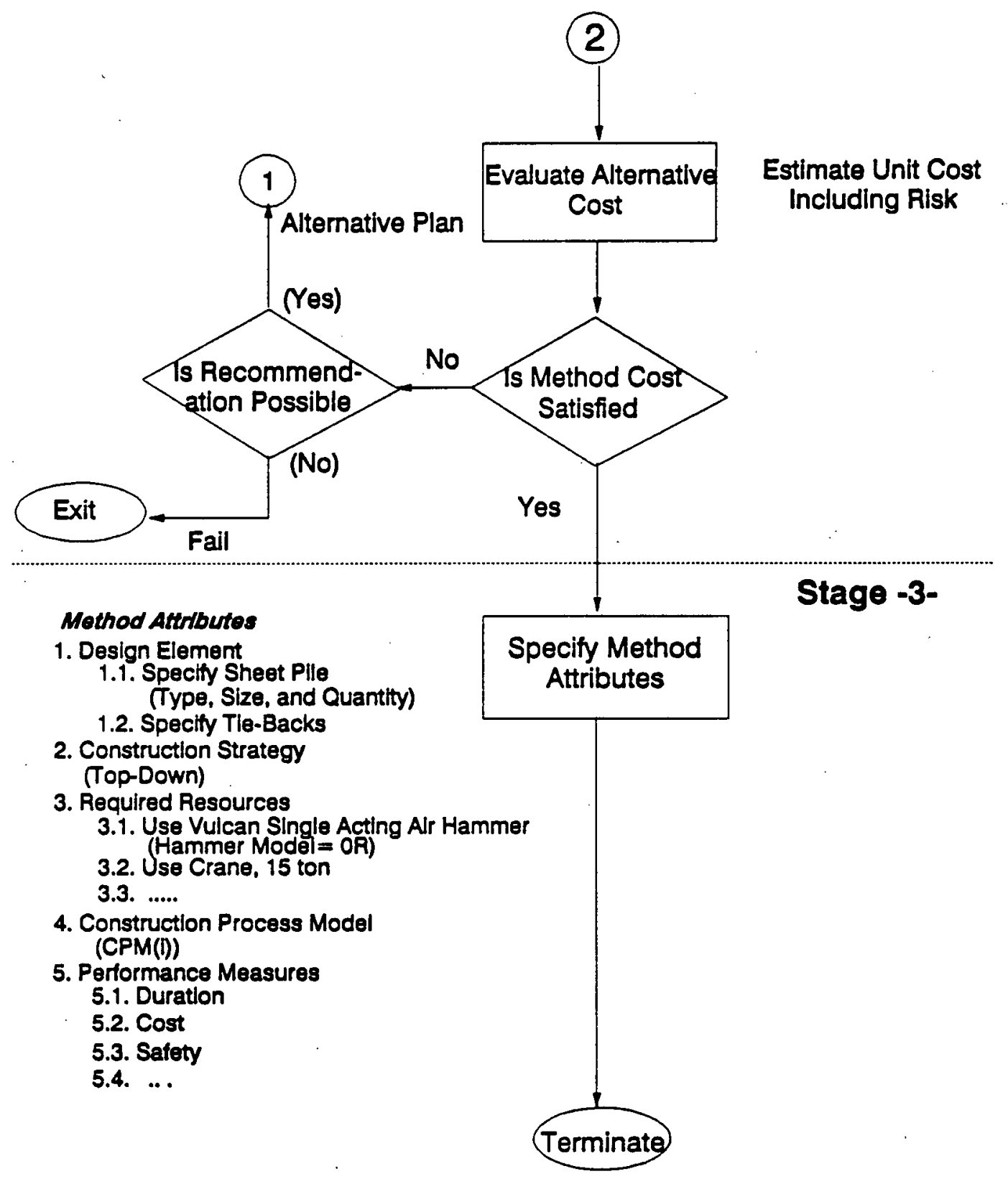


Permanent Structure

Purpose:

Dimensions:

Site Conditions

Location:

U/G Utilities:

Tiebacks:

Soil Conditions

Soil Type:

Water Table:

Design Constraints

Retaining System:

Displacement:

\section{Project Goals}

GWSS

Target production rate (tpr):

Target cost (tc):

Excavation

Target production rate (tpr):

Target cost (tc):
Utilities Tunnel.

$\mathrm{L}=1200 \mathrm{ft} ; \mathrm{W}=20 \mathrm{ft} ; \mathrm{D}=20 \mathrm{ft}$.

Downtown urban area.

None

Easement available

Soft clay

15 feet below surface elevation

$<12$ feet below retaining system.

No more than 3 inches.

30 feet/day

$\$ 15.00 / \mathrm{ft}^{2}$

$30 \mathrm{feet} /$ day

$\$ 20.00 /$ cy

\section{Table 4.2 Gws Project Context Data}

This information is used to help identify and screen possible methods. At the outset, high level information is sought. Once a detailed examination of methods is performed, more low level, detailed information is requested from the user. The dialogue is meant to identify the information required and its influence on the decision process. The context information is requested in the form of rules. 


\section{step 2}

The selection process starts with a classification of the methods alternatives known to the system, (i.e. stored in its data base), which provides a representation or categorization scheme for all methods such that other desired or new methods could be entered by the user. A methods data base thus contains a library of existing methods characterized by a set.of dimensions, including method attributes and pertinent factors such as level of risk, safety, resources compatibility, level of expertise, and so forth: i.e. a method associated with specific knowledge. Specifications for a method database is not part of this thesis.

We come out of the preliminary screening with a set of possible design alternatives:

$$
D A=\{D A(1), D A(2), \ldots D A(n)\}
$$

and a set of possible construction strategies:

$$
\operatorname{cs}=\{\operatorname{cs}(1), \operatorname{cs}(2), \ldots \operatorname{cs}(n)\}--
$$

where links between the DA's and the CS's may exist. Allowance is made for the user to augment the list of design alternatives and add to the knowledge base.

User context input is applied to eliminate alternatives and rank those that are feasible. This process uses knowledge in the form of rules for this task. The standard 
form adopted for the formulation of a design alternative (DA) feasibility rule is as follows:

RULE Design Alternative (i) Is Technically Feasible

IF [ DA(i) Technical Feasibility Conditions Are Satisfied ]

AND [DA(i) Time/Cost Performance Conditions Are Satisfied]

THEN [DA(i) is Feasible ]

Technical feasibility refers to the ability of the design alternative (or construction strategy or resource assignment) to satisfy project specifications, regulatory constraints, work space constraints, etc. Each of the elements of this rule format may correspond to the evaluation of a set of rules.

An example of a simplified rule for filtering GWSS alternatives, for the steel sheet pile alternative to be feasible at this stage, is as follows:

$\begin{array}{ll}\text { RULE } & \text { SSP (DA(1)) Is Feasible } \\ \text { IF } & \text { Tunnel Depth }<20 \text { feet } \\ \text { AND } & \text { Soil Condition = Soft Clay } \\ \text { AND } & \text { No U } / \text { G Utilities = True } \\ \text { AND } & \text { lbpr } 1<\text { tpr }<\text { ubpr } 1 \\ \text { AND } & \text { lbc1 }<\text { tc }<\text { ubc1 } \\ \text { THEN } & \text { DA }(1)=\text { Steel Sheet Pile } \\ \text { AND } & \text { Steel Sheet Pile is Feasible }\end{array}$

"lbprl", "ubprl", "lbcl", "ubcl" are the lower and upper bounds of production rate and cost ranges for the methods design alternative level, given a specific project context. 


\section{step 3}

Construction strategy for the GWS could be discussed at two levels: high, or project, level of Top-Down versus BottomUp; and low, or activity, level of retaining system type of tie-backs versus wales and struts. The first drastically alters the make up and sequencing of major tunnelling activities, and subsequently the method attributes. The second is central to excavation activity where the tie-backs retaining system speeds up excavation where this activity is a major one for cut-and-Cover tunnelling.

For our example, we started with a GWSS design alternative of steel sheet piles which implicitly embraces a top-down construction strategy. However, when a GWSS slurry trench wall is considered, and ground surface restoration is regarded as a primary concern, then of the two construction strategies, the bottom-up will be preferred. Its choice eliminates the SSP alternative and other traditional GWSS alternatives. Further low level construction strategies for pile driving patterns, i.e., in waves, doubles, or singles, etc., may be considered.

The standard form for the rule that determines feasibility of a construction strategy is as follows.

RULE [Construction Strategy (j) + Design Alternative (i)] Is Feasible

IF [CS(j) COMPATIBLE with DA(i)]

AND [CS(j) Technical Feasibility conditions Are Satisfied ]

AND $\quad[\mathrm{CS}(\mathrm{j})$ Time/Cost Performance conditions Are Satisfied ]

THEN [CS(j) + DA(i) Is Feasible $]$ 
The rule defines compatibility as the ability to combine a specific design alternative with a specific construction strategy.

Excavation could be a bottleneck activity, therefore we have two feasible choices, $\operatorname{cs}(1)$ and $\operatorname{cs}(2)$, of tie-back and anchorage retaining system, that are feasible for DA(1) of steel sheet pile, i.e. either $\operatorname{CS}(1)+D A(1)$ or $\operatorname{CS}(2)+$ $\mathrm{DA}(1)$. Since minimizing time is the prime goal ( a hypothesis made for discussion purposes), the first choice will be favored, given an easement permitting tie-backs is possible, allowing more freedom of resource movement and feasible resource combinations.

Should all strategies fail to be feasible, then the control system selects the next preferred design alternative. A new set of construction strategies appropriate for the selected design alternative is examined. Determination of a feasible strategy at this stage passes control on to the next stage.

\section{Step 4}

Key resources are selected from a data base of resource alternatives. The guiding principle in assigning resources is that the largest capacity equipment that satisfies space and/or availability constraints is selected, in order to maximize the production rate, and reduce production risks. 
If no feasible resource combination exists then the plan fails and the system backtracks to previous stages to alter the plan. The standard form of the rule for resource assignment parallels that for the design alternative level. An example rule which reflects the interdependencies between work packages is as follows.

$\begin{array}{ll}\text { RULE } & \text { Select Resource Class for Excavation } \\ \text { IF } & \text { Activity }=\text { Excavation } \\ \text { AND } & \text { Geometry }=\text { Class } 1 \\ \text { AND } & \text { DA[GWSS }=\text { DA }(1) \text { Steel Sheet Pile }\} \\ \text { AND } & \text { CS }[\text { GWSS }]=\text { CS }(1)\{\text { Tiebacks }\} \\ \text { AND } & \text { lpr }\{\text { Excavation }<<\text { Required Excavation Rate }<\text { upr }\{\text { Excavation }\} \\ \text { THEN } & \text { Excavation Resource }=<\text { backhoe class }> \\ \text { AND } & \text { Set }<\text { backhoe class }>=\text { Cat } 245\end{array}$

For our example, a shallow tunnel, this corresponds to selecting the largest piece of equipment which satisfies the technical feasibility constraints, for example, a Caterpillar Hydraulic Backhoe 245 from the set of 245,235 , 225, 215 models (Caterpillar 1982). Similarly, the steel sheet pile installation activity requires specifying a pile driver and a crane resources.

At the end of phase 1, the attributes of one or more feasible methods are passed to phase 2, detailed feasibility, for further manipulation.

\section{step 5}

This step is directed at specifying the representation and analysis methods, given specification of the design 
alternative, construction strategy and resource assignment at the detailed level.

The design alternative is further specified in terms of its structural members and its retaining system such as soldier piles, sheet piles, trench wall, shotcrete, wales, anchorages, struts, etc. Resources are sized. For example, a resource for excavation such as the Cat 245 backhoe class suggested by phase 1 (see previous rule) is further specified as having a $30 \mathrm{ft}$, one-piece boom with a $14 \mathrm{ft} 6$ inch stick, $21 / 2$ cu bucket, etc., attributes that are pertinent to its production. Construction strategy decisions include specifying vertical and horizontal spacings, and other construction cycle decisions (time and space lags of activities), and construction model processes.

Construction Process Model (CPM) refers to a feasible combination of construction resources under a project context (including geometry) that can be modelled via simulation, dynamic programming, and other oR techniques, or mimicked by previous experience, to predict their performance measures. Representation formats include networks, line of balance charts, and simulation models. other knowledge incorporated in this stage deals with the degree of definition appropriate to the modeling task, for example number of activities, time/space lags, and so on. 
At stage 1, an attempt is made to marry method attributes variables under an appropriate construction model process scheme or scenario.

If GWSS DA(i) installation and excavation (the retaining system spacing is implicitly incorporated in the construction cycle design) are separable, then each construction process model is evaluated separately, and the project progress will be controlled by the slowest pace of either. If the GWSS DA(i) is soldier pile and lagging, then the lagging and retaining system installation are intertwined with excavation. For intertwined activities, one construction process model is used to collectively predict their performance measures.

For our example of steel sheet pile, the GWSS installation and excavation are intertwined if they share a common multi-task resource such as the crane, which can be used to load/unload muck and construction materials, and hold/move the pile driver. If separated, then steel sheet pile installation process will be modeled by the hammer energy formula which predicts the productivity rate of pile driving based on the soil/pile/hammer scenario.

The retaining system installation is always intertwined with excavation as discussed in section 3.7 .2 , and thus implicitly incorporated. 
A simplified rule which is more applicable for soldier piles and lagging (not applicable (N/A) for steel sheet piles since the GWSS installation and excavation are separate) is as follows:

$\begin{array}{ll}\text { RULE } & \text { Select/Assign Process Model for DA(1) and CS(1) } \\ \text { IF } & \text { GWSS Installation Activity AND Excavation Activity = Intertwined } \\ \text { AND } & \text { Excavation Resources = Backhoe_Cat_245 } \\ \text { AND } & \text { Retaining System Resources =Crew_B } 50 \\ \text { THEN } & \text { Assign CPM }=\quad \text { Process.GWSS }\{\text { DA(i), CS(j) + Process } \\ & \text { Fxcavation }\{\mathrm{DA}(\mathrm{m}), \mathrm{CS}(\mathrm{n})\} \\ \text { AND } & \text { Find Performance Measures }\end{array}$

The goal here is to guide the user in conducting an analysis through the use of rules. Standard models would be predefined so as to automate as much of the construction model process formulation as possible.

\section{step 6}

Control is then transferred to the process models which are executed and the results interpreted (stage 2 and stage 3 ). If the predicted production rate and cost are below the required rates, possible changes to the process model or resource assignment may be suggested to the user so that model changes can be made. If satisfactory time performance cannot be obtained, then the control system attempts to direct the search back to the first or second stage search processes. Given satisfactory time performance, the process is repeated for cost performance. The total process is 
completed by assigning attribute values to the methods frame (stage 4).

The control strategy for the above generic system was described using pseudo rules for illustrative purposes. They are not rigorous rules designed for implementation.

\subsection{CMSA Development}

\subsubsection{Overview}

The scope of the prototype system is described in this section. Central to the development of a methods selection system are representation issues as they relate to context, design alternatives, construction strategies, resource and process models, development of suitable control strategies and treatment of feasibility criteria. Effective approaches for dealing with these topics constitute the basic building blocks of the system. Thus, attention has been restricted to showing how a typical element at each level in the decision making hierarchy can be modelled, and how both the declarative and procedural knowledge required to generate the attributes/decision demanded at each level can be represented.

In particular, a very restricted set of context, design, construction and resource alternatives have been selected for examination for the ground wall support system design element for Cut-and-Cover tunnelling. A description of two soil geotechnical contexts provides insights into the 
representation of project context.. Steel sheet piling has been chosen for the design alternative. Driving in singles or pairs has been selected for the construction strategies. The selection of a pile hammer provides the focus for resources, and determination of pile driving rate constitutes the process model.

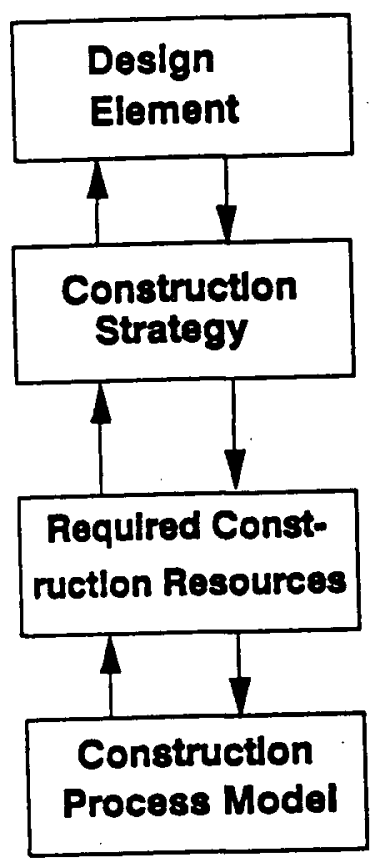

(a)

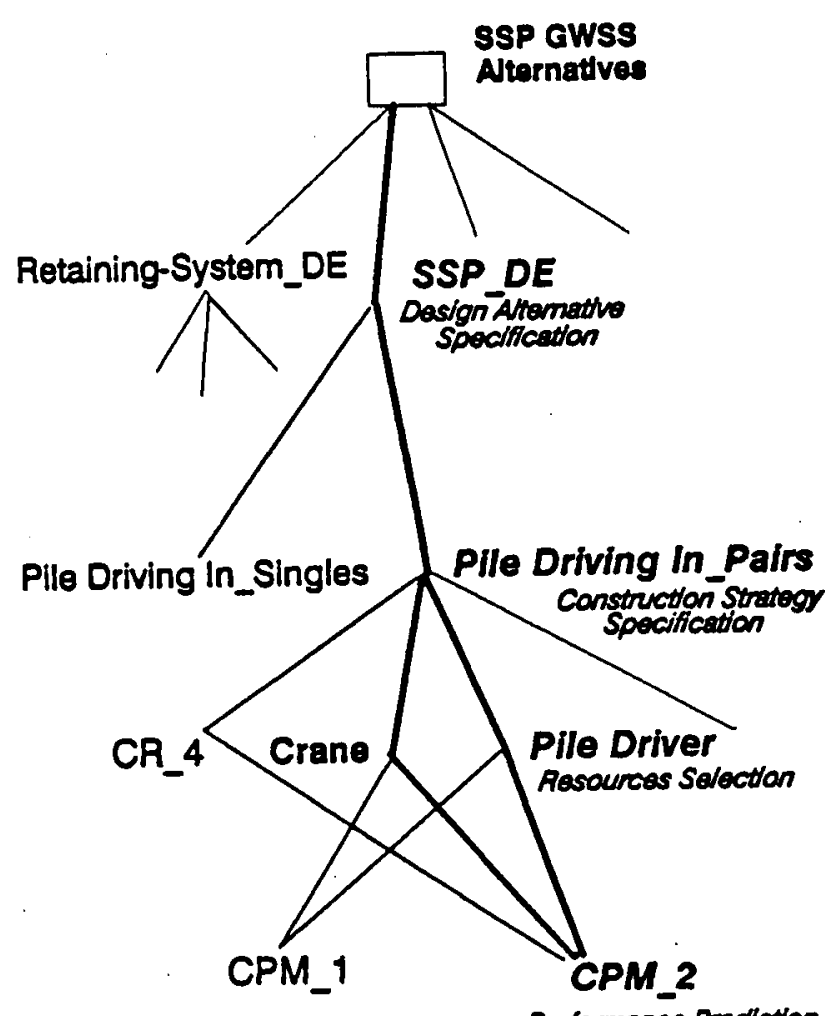

Partomances Predlction (Apoly Dynamic Formula)

Figure 4.5 steel sheet Pile (88P) Method Frame 
This path through the decision alternatives at each decision level is illustrated in figure 4.5. As described in section 4.2 , a two stage process has been adopted as the basic decision model. This process is depicted in figure 4.6. Preliminary feasibility is charged with the task of providing a short list of potentially feasible alternatives, while detailed feasibility has the task of confirming feasibility and assigning the attributes of the design, construction strategy and resource alternatives selected.

At the preliminary feasibility level, broad brush rules are used to select and prune alternatives, based on an assessment of various criteria which are encoded in a set of rules. This set corresponds to knowledge base 1 (KB-1) in figure 4.6, where knowledge bases at both levels are partitioned according to their corresponding criteria: risk, design, construction resource compatibility, construction performance, and regulatory. While its features are described in section 4.3 .3 , it has not been implemented in the current work. Instead, implementation efforts have concentrated on defining and implementing the knowledge bases at the detailed feasibility level shown in figure 4.6. 


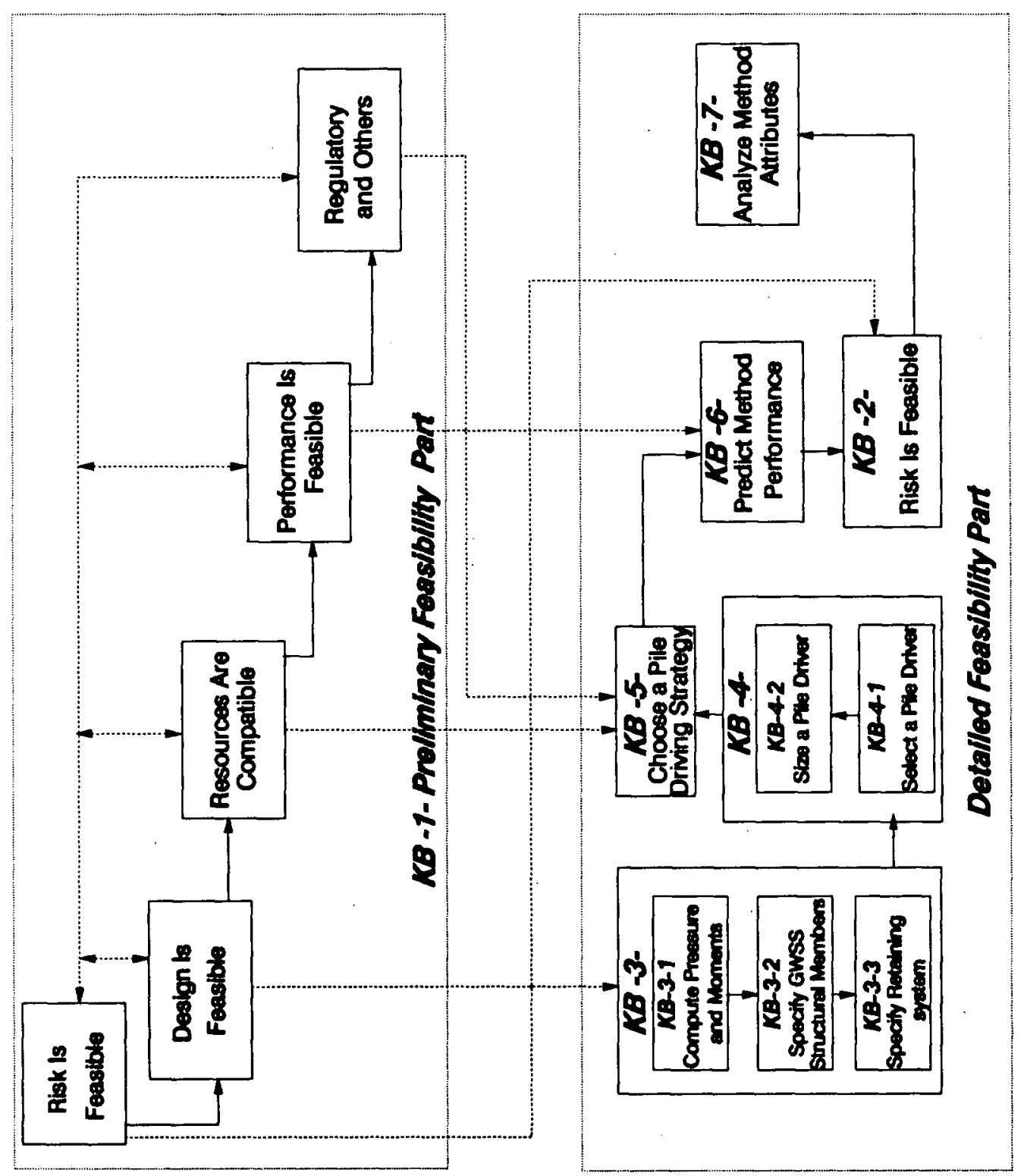

Figure 4.6 Prototype Model 
This section is organized as follows. In section 4.3.2, representation of the project context in terms of soil conditions is examined. In section 4.3.3, a descriptive model of the preliminary feasibility stage is presented. In section 4.3.4, the detailed feasibility model is described for the alternatives previously identified.

The presentation is organized around subheadings of context, criteria, control strategy and knowledge representation. Because the notion of a frame is a useful way of identifying the attributes of a construction method, a conceptual frame is used throughout to demonstrate the build-up of the method attributes through preliminary, then detailed, feasibility.

\subsubsection{Context Modelling}

Context modelling, or representation, consists of two parts. The first deals with the representation of the initial site conditions, contractual conditions, and so forth. The volume of information that has to be elicited from the user can be substantial, leading to design issues regarding the user interface. For example, should information be requested only if needed by a rule, or should a comprehensive description of project conditions be input first (a potentially onerous burden for the user). For the prototype work, the latter approach has been adopted. 
The second part of the context modelling deals with the derivation of additional context information through the application of engineering knowledge (e.g. lateral pressure) and exploration of tentative decisions regarding construction method attributes (e.g. space requirement for heavy equipment). Both of these aspects are examined below by way of the modelling of soil conditions, which have a significant affect on decisions relating to the selection of the ground wall support system and pile driving productivity.

To have a small subset of soil types for the prototype system, four soil types were treated: soft clay, hard clay, loose sand, and dense sand. For each type, a data base of properties (dry unit weight, submerged unit weight, unconfined compressive strength, angle of shearing strength, etc) was developed.

A maximum of two soil layers was treated (see figure 4.7). To gain a sense of how many soil profile scenarios could be generated by these four soil types and two layer model, consider this: for a single layer model, four options exist: soft clay, loose sand, etc; while for a two layer model, 12 combinations of conditions exist: "soft clay on stiff clay", "soft clay on Loose Sand", etc. 


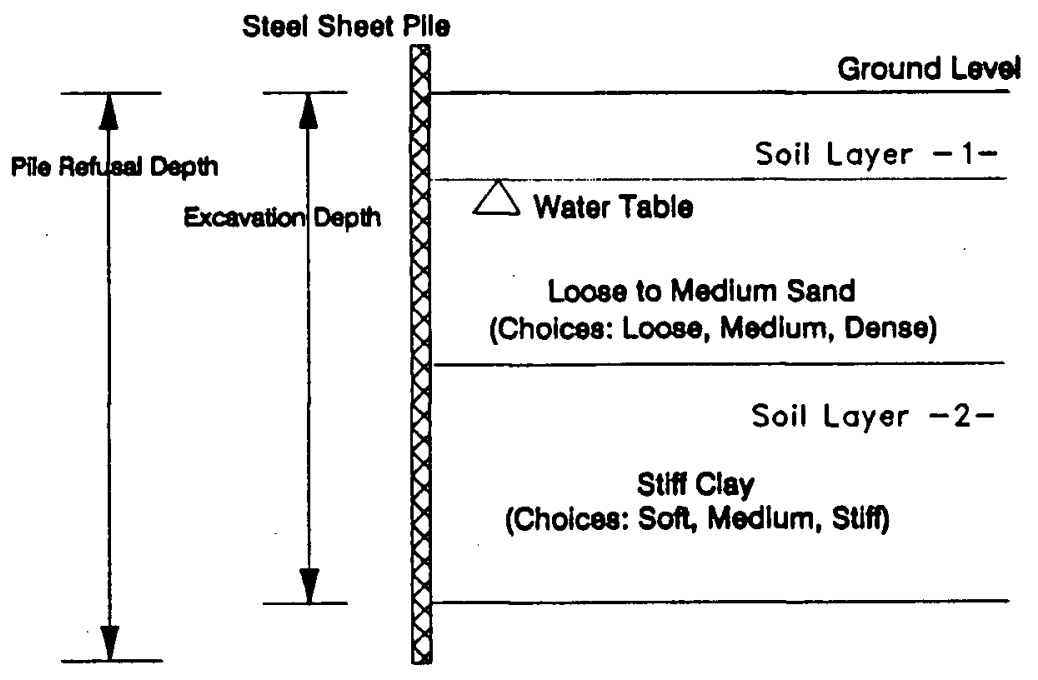

(a) soil profile with Properties of Each Layer Entered by the User

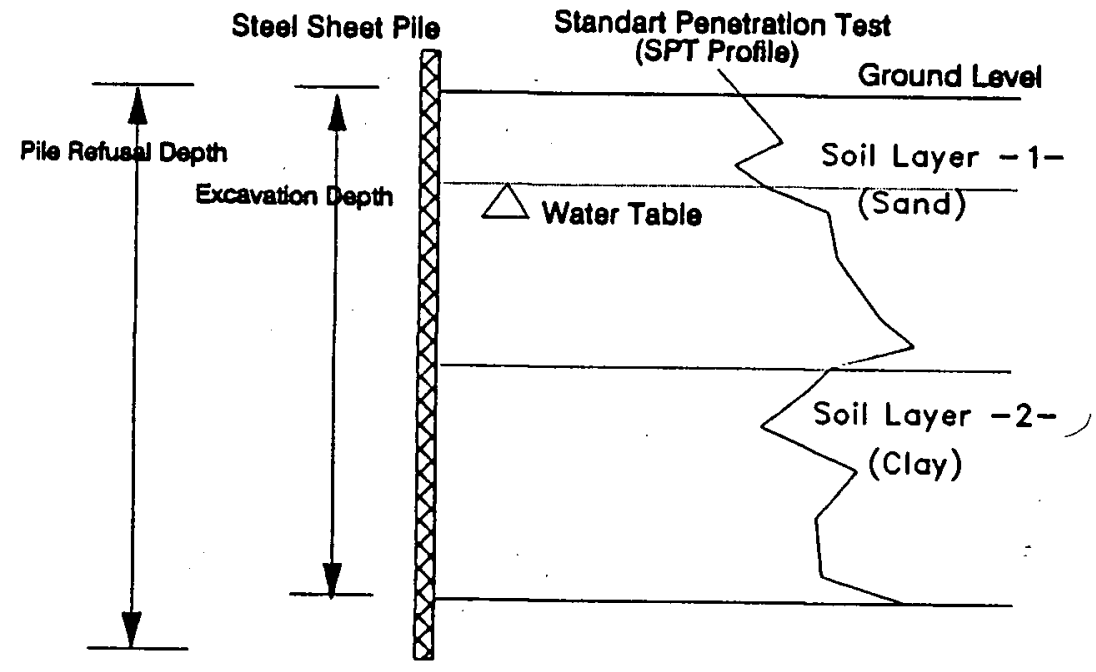

(b) soil profile with spr profile for sand and clay

\section{Figure 4.7 soil Profile scenarios}

Thus soil profile combinations grow exponentially in size with the number of soil types and soil layers. The context model developed was rich enough to demonstrate the dependance of methods selection decisions on context 
variables. For example, the order and depth of each soil layer significantly influences the selection of the hammer as shown in section 4.3.4. The soil profile also affects the pace of pile driving as incorporated in the hammer energy formulas.

The two approaches explored for modelling soil properties are described below.

\section{Layer by Layer Property specification}

The user is queried as to the properties of each layer and the placement of the tunnel structure within the layers. Example data required by the prototype CMSA system are shown in table 4.3 .

$\begin{array}{ll}\text { Soil_Type_1 for Layer_1 } & =\text { Loose_Sand } \\ \text { Depth of Layer_1, } & =40 \mathrm{ft} \\ \text { Unit Weight of Soil_Type_1 } & =100 \mathrm{pcf} \\ \text { Wet Unit Weight of Soil_type_1 } & =120 \mathrm{pcf} \\ \text { Submerged Weight for Soil_Type_1 } & =38 \mathrm{pcf} \\ \text { Angle of Shearing Strength for Soil_Type_1 } & =30 \\ \text { Unconfined Comp. Strength for Soil_Type_1 } & =\mathrm{N} . \mathrm{A} . \\ \text { Depth of Water Table, } & =20 \mathrm{ft} \\ \text { Depth of The Tunnel or the Excavation } & =60 \mathrm{ft} \\ \text { Soil_Type_2 for Layer_2 } & =\text { Soft to Med. Clay } \\ \text { Depth of Layer_2 } & =17 \mathrm{ft} \\ \text { Unit Weight of Soil_Type_2 } & =111 \mathrm{pcf} \\ \text { Submerged Weight for Soil_Type_2 } & =52 \mathrm{pcf} \\ \text { Angle of Shearing Strength for Soil_Type_2 } & =\mathrm{N} . \mathrm{A} . \\ \text { Unconfined Comp. Strength for Soil_Type_2 } & =120 \mathrm{pcf}\end{array}$

Table 4.3 soil profile Input-Format 1

\section{Soil Penetration Test (BPT) Model}

Figure 4.7 (b) shows two soil layers identified as sand and clay. Indirect relationships exist among the standard 
Penetration Test (SPT) profile, soil resistance and soil properties for each type of soil layer.

Using these relationships, CMSA infers soil properties through a data base of SPT profile versus soil properties for a soil type.

A sample of soil information required by CMSA as provided by the user for the SPT model is shown in table 4.4 . It is elaborated upon in table A.I in Appendix A.

$\begin{array}{ll}\text { Soil_Type_1 of Layer_1 } & =\text { "Loose_Sand" Sand } \\ \text { Depth of Layer_1 } & =40 \mathrm{ft} \\ \text { Soil_Type_2 of Layer_2 } & =\text { "Stiff_Clay" Clay } \\ \text { Depth of the Tunnel_ } & =65 \mathrm{ft} \\ \text { Depth of Water Table } & =17 \mathrm{ft} \\ \text { SPT at depth } 10 \mathrm{ft} & =30 \\ \text { SPT at depth } 20 \mathrm{ft} & =20 \\ \text { SPT at depth } 30 \mathrm{ft} & =21 \\ \text { SPT at depth } 40 \mathrm{ft} & =11 \\ \text { SPT at depth } 50 \mathrm{ft} & =15 \\ \text { SPT at depth } 60 \mathrm{ft} & =30 \\ \text { SPT at depth } 70 \mathrm{ft} & =33\end{array}$

Table 4.4 Soil Profile Input-Format 2

Once a soil model is selected and parameter values specified by the user, additional context information essential to the selection of design alternatives, construction strategies and resources can be generated. Most of this information can be derived using procedural knowledge. The routines used for determining lateral earth

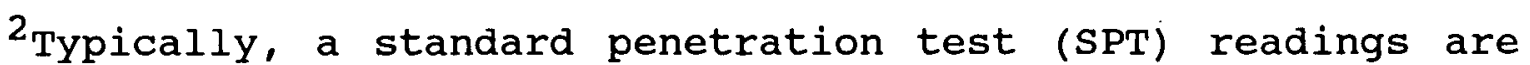
taken at $3 \mathrm{ft}$ or $5 \mathrm{ft}$ intervals to the refusal depth of the pile. Here, we approximate the readings interval as every $10 \mathrm{ft}$. 
pressure and moments for the ground wall support systems are described in Appendix A. The output from these routines forms an important part of the technical feasibility check at the design alternative level.

Appendix B contains the derivation and the code for a prediction algorithm to forecast the pile driving productivity rate and check if pile refusal depth has been achieved. The value of this approach was confirmed through interviews with personnel from a pile driving company (see Appendix C).

\subsubsection{Preliminary Feasibility}

Figure 4.8 presents a way of prescribing a GWSS construction method frame throughout feasibility synthesis over time: a frame builds up incrementally, while slots are instantiated and added over time. This figure alludes to the two levels of feasibility where primary and secondary variables are evolved.

GWSS methods will be retrieved from a data base characterized by universal attributes (slots) corresponding to method elements and knowledge attached to them. A GWSS attribute contains technical feasibility, criteria, knowledge, context, and control strategy, and is subjected to the feasibility checks of risk, design, compatibility, construction performance and regulatory environment. Not 
all of these factors are applicable for a GWS attribute and/or project scenario.

The preliminary knowledge base, $\mathrm{KB}-1$ in figure 4.6, identifies, screens, and ranks, feasible alternatives at this level, and then passes control to the detailed feasibility level for further method specification.

The goal is to reduce the solution set as quickly as possible by treating various feasibility criteria in the form of high level rules. The knowledge here is declarative, based mainly on experiential and common sense engineering knowledge.

This knowledge, in the form of rules, is attached to each method alternative in the data base. Condition variables in these rules are assigned values based on context parameter values, and then the rules are evaluated. 


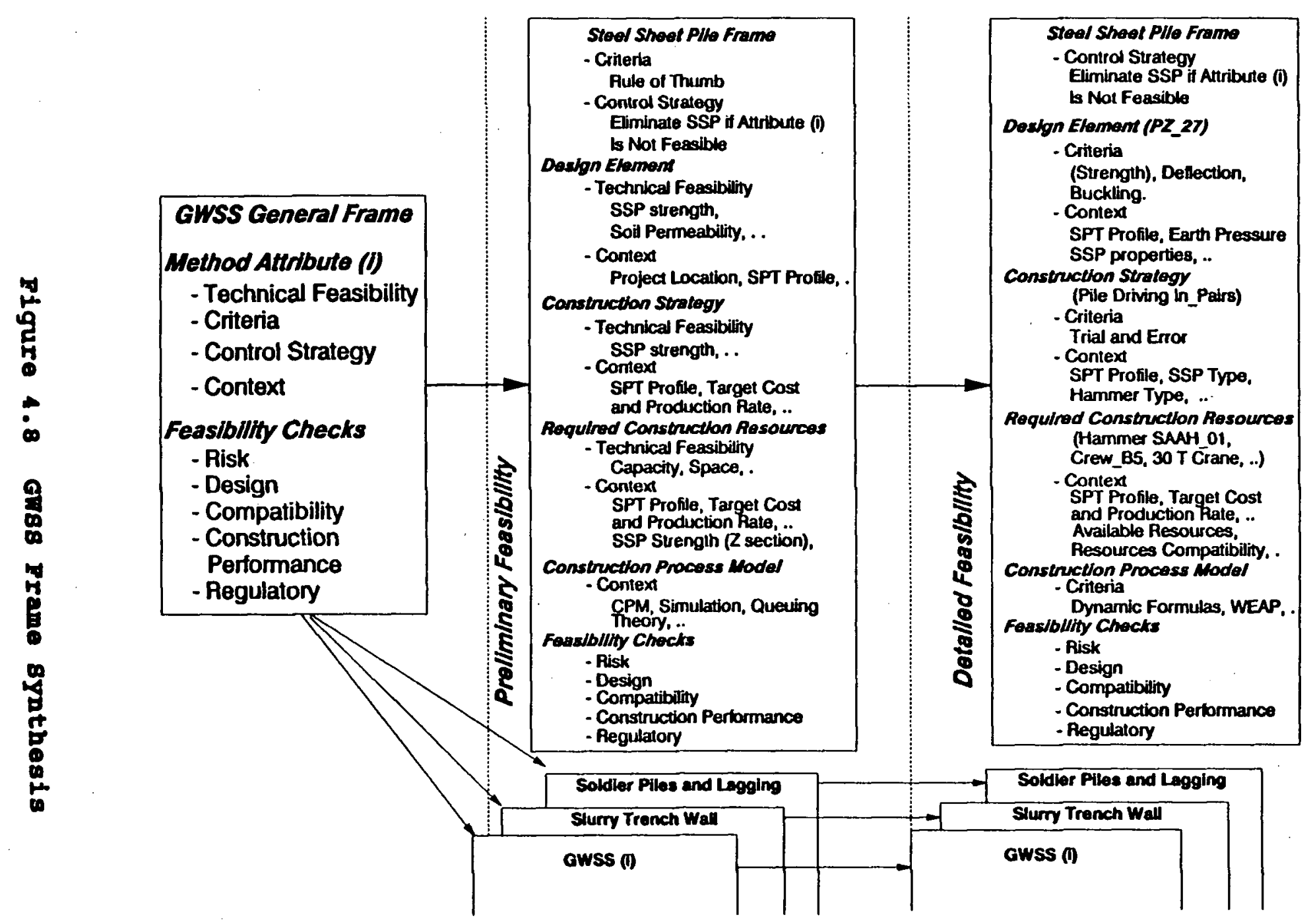


The control strategy at this level may pass control vertically to a lower level component or horizontally to another rule category when necessary. For instance, for a given shoring alternative and project context, the risk component may be processed first, followed by design feasibility, construction resources feasibility, performance feasibility, and so forth. Another alternative with the same or different project context may dictate checking the design first, followed by performance measures test, and others. In other words, the search strategy is sensitive to project context and user intervention .

For CMSA, KB-1 initiates the solution process by testing the preliminary feasibility of the risk component. After satisfying the risk requirement, control passes to the design component. If the user wants to override this, he instructs the system to pursue further low level risk analysis. Then design, construction resource compatibility, construction performance, and regulatory feasibility are checked, subject to user/project context intervention to redirect control.

High level rules are used to represent criteria knowledge for each alternative. A rule consists of a RULE heading to describe the rule, IF conditions that represent high level premises (which may embody a another layer of rules), and THEN consequences or actions that take place if this rule is 
fired. Control strategy clauses are labelled to indicate passing of control. This highlights passing the control in either direction: horizontally within one level, and/or vertically between the two levels of feasibility as shown in figure 4.6 .

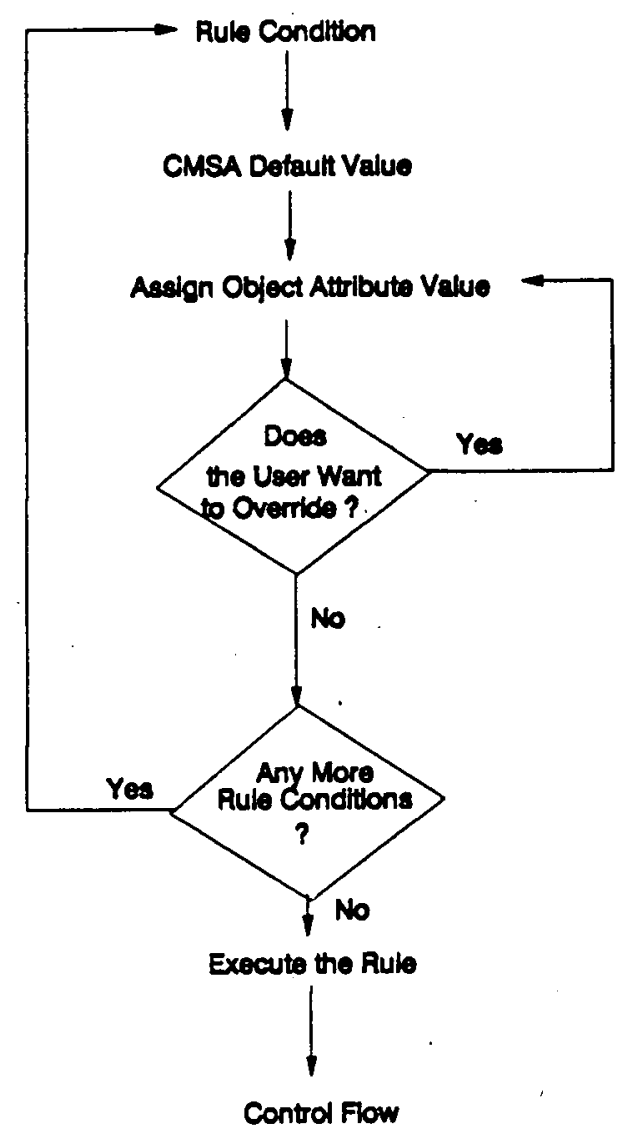

Figure 4.9 CMsA Rule Execution Loop

Figure 4.9 shows a typical CMSA rule execution order. Each condition (or premise) must have a value, where some 
parameters' values are provided by the system, subject to user override, while others must be provided by the user. once a rule is fired, then, some conclusion clauses fire to perform another operation and/or pass control to another hypothesis. For instance, if a rule in the preliminary feasibility failed, then CMSA is supposed to stop the session, however, the user may override this and command the system to pursue the solution.

We illustrate and describe each feasibility check for each rule category for our steel sheet Pile, GWS design alternative. The SSP alternative is presumed to exist in the methods database with pertinent knowledge for each feasibility check.

A risk factor is first applied before further refined information is required from the user for other factors. The system evaluates the SSP alternative risk-wise by checking variable conditions such as the state of water washout. These conditions are either volunteered by the user, or provided by the system as a system default, database, context, etc. A typical rule for risk feasibility is shown below. 
1. Risk Is Feasible

$\begin{array}{lll}\text { RULE } & \text { Risk_Is_Feasible for A GWSS } & \text { Source } \\ \text { IF } & \text { GWSS is Steel_Sheet_Piles } & \text { Database/User } \\ \text { AND } & \text { Water_Level/Tunnel_Depth Is High } & \text { User } \\ \text { AND } & \text { Water Washout.Risk Is Low } & \text { Context, User } \\ \text { OR } & \text { Local_Expertise Is Medium } & \text { User } \\ \text { AND } & \ldots & \\ \text { - } & & \\ \text { Hypothesis } & \text { Expected_SSP.Risk = < Catastrophic.Risk } & \\ \text { THEN } & \text { Steel_Sheet_Pile.Risk Is Acceptable } & \\ \text { ELSE } & \text { Steel_Sheet_Pile.Risk Is Not_Acceptable } & \end{array}$

\title{
\{Control Clauses if Acceptable\}
}

\author{
AND/OR Check Design_Feasibility \\ AND/OR Check Detailē__Detailed for Risk Analysis \\ AND/OR Check Performance Measures Are Feasible
}

\{Control Clauses if Not_Acceptable\}
AND
Eliminate SSP
AND
Go to Next GWSS Alternative

The premises of this rule, and other rules to follow, are used to perform the feasibility check. The premise conditions are high primitives and expect a boolean answer of YES or NO. If any one of these conditions fails, then the rule fails, and ELsE of the control (strategy) clause decides what to do next. For instance, the above rule reads that Water_Washout.Risk_Is_Low implies that the user has answered YES to the question "Is there a Low_Risk for a water_washout ?". If the answer is YES for this clause and other IF clauses, then Steel_sheet_Pile.Risk is Acceptable. The system keeps the boolean value of this rule as TRUE. If the same clause of the Risk. level was not low, then the rule 
fails and it is the task of the control strategy to decide what to do next.

Multiple values for the state of water washout such as Medium_Risk, or High_Risk, where it jeopardizes GWSS feasibility, may be considered by evoking risk rules which contain these values in their premises.

The Local_Expertise state is treated in the same manner. This is a high level primitive clause of great importance in assessing the risk. Interviews with a contractor (Appendix C) indicated that local practice is very important when assessing risk. Even if a method is proven and well known elsewhere, if local expertise is not available, the method is perceived as having high, and potentially unacceptable, levels of risk. This argues strongly for using methods familiar to a firm's practices. This is evident in the interview section of Appendix $c$, in which a contractor indicated shot-crete is a common shoring method in Vancouver, whereas, in the Toronto area, $\mathrm{H}$ piles and lagging are more prevalent. Although this clause, Local_Expertise conditions, may deserve a component by itself, it was combined together with the risk component for knowledge organization. It is not the intent of this thesis to identify and analyze its attributes.

If the risks associated with the method are feasible, then control is transferred either horizontally, to check 
other components, or vertically, to further check detailed level risk assessment if the user chooses to. If the alternative is found to be unacceptable (infeasible), then SSP is eliminated and control picks another candidate. The design feasibility at this level is basically technical. That is, given project and site conditions, is the design feasible with regard to leaving adjacent structures undamaged, testing its constructability, etc.

A representative rule for design feasibility is exemplified next.

2. Design_Alternative Is_Feasible

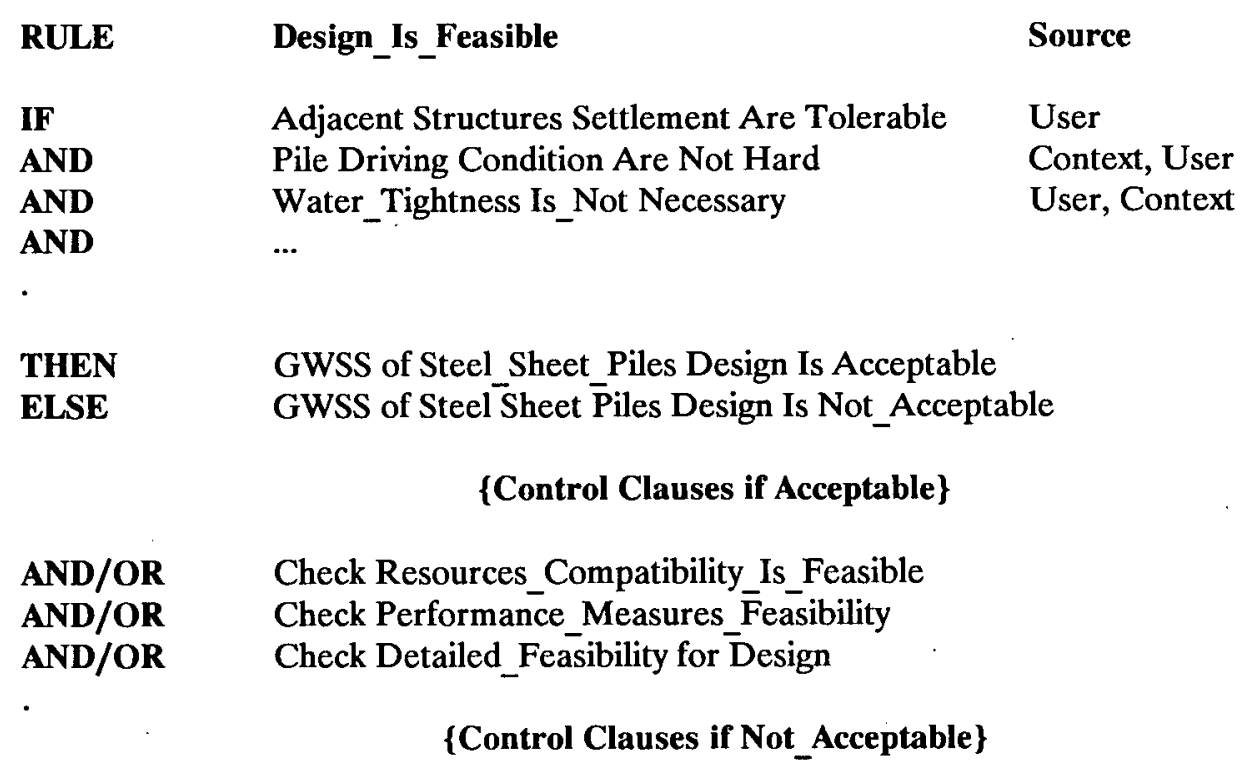

$\begin{array}{ll}\text { AND } & \text { Eliminate SSP } \\ \text { AND } & \text { Go to Next GWSS Alternative }\end{array}$

The first premise examines whether settlement of the adjacent structures to the shoring GWSS is permissible, i.e. tolerable. The user is assumed to volunteer the answer. 
The pile driving conditions are essential since SSP are installed by this technique. Therefore, it is desirable to satisfy this condition. The third premise tests the importance of water tightness which depends on the water table level. For some projects it is important to have water tightness, while others do not stipulate it. In this case, this condition is phrased such that water-tightness has to be satisfied. The source of this information could be either the user or the context, whereby the system presumably assesses the water table elevation versus project conditions and returns an answer.

The above rule checks the feasibility of the sheet piles design (or other GWSS) within a given project context. Further, if this rule is fired successfully, control then may be transferred to check horizontal components or proceed to a lower level of detailed design.

Next, control passes to check the resource compatibility for the given design alternative, where available equipment and crew spreads are identified and balanced for smooth production.

The following rule demonstrates a compatibility check. 
3. Resource Compatibility Is Feasible

$\begin{array}{llr}\text { RULE } & \text { Resource_Compatibility_Is_Feasible } & \text { Source } \\ \text { IF } & \text { Soil Profile Is Cohesive } & \text { Context, User } \\ \text { AND } & \text { Required Hammer Energy }>33,000 \mathrm{lb} \text {. ft. } & \text { Context, User } \\ \text { AND } & \text { SSP_Designation Is PZ_27 } & \text { Context, User }\end{array}$

THEN (Hammer Size + SSP Size + Soil Profile)

Is Technically Feasible User, Context

AND Hammer_and_SSP Are Compatible

\{Control Clauses if Accepted\}

AND Check Detailed_Feasibility for Resource_Compatibility

AND Check Performance_Measures_Feasibility

\{Control Clauses if Not_Acceptable\}

AND Eliminate SSP

AND Go to Next GWSS Alternative

Resources, including design materials, must be compatible. For instance, some materials are only constructed with a subset of existing tools and/or equipment, or vice versa, where some materials have to be substituted to suit existing resources, such as crew expertise and available equipment.

In this vein, the first premise in the above rule pronounces the soil context condition to be cohesive soil. The second premise dictates a lower bound for the hammer power. This is provided by the context which draws from a lower level rule that relates the likelihood of SPT bring greater than a threshold and minimum hammer energy, thus defining a feasible hammer subset. The third premise 
identifies the type of SSP section as PZ sections, which are heavy and have a high section modulus. This is based on the context or user volunteered information. For the former, an implied relationship between the pile driving conditions, and/or hammer size, versus SSP minimum size, is established, reducing the set of feasible SSP.

The object is to reduce set of available lists of hammers and SSPs into reduced feasible subsets that satisfy expected goals, e.g. cost and time.

Control passes horizontally to check the performance measures feasibility. If the horizontal inference carries on, the next performance feasibility will be checked at this stage. This component establishes upper and lower bounds for production and costs from previous projects, and/or unit cost data manuals, given for a project context scenario including soil profile and equipment/material spread. The following rule mirrors this component function. 
4. Performance Measures Feasibility

RULE Performance Measures Are Feasible Source

IF Lower_Prod._Bounds $<$ Production_Rate $<$

$\begin{array}{lll}\text { AND Upper_Prod.BBounds } & \text { User } \\ \text { Lower_Cost_Bounds }<\text { Production_Cost }< & \\ \text { Upper_Cost_Bounds } & \text { User }\end{array}$

AND $\ldots$

THEN Steel_Sheet_Piles.Performance_Measures Are Feasible

\{Control Clauses if Accepted\}

AND Check Detailed_Feasibility for Other Components

AND/OR Check Detailed_Feasibility for Performance_Measures

...

\{Control Clauses if Not_Acceptable\}

AND Eliminate SSP

AND Go to Next GWSS Alternative

These upper and lower bounds are goals (stated in the contract as unit cost and construction duration), and the user is supposed to estimate the Production_Rate and Production_cost for a given design alternative, and construction resources and processes. At this level, the estimates are conceptual, a rule of thumb based on experience from a project context, whereas further precision of estimates could be done at the lower level.

Control then passes to the regulatory component to check compliance with regulatory and safety requirements. An example is presented in the next rule. 
5. Regulations Are satisfied

$\begin{array}{lll}\text { RULE } & \text { Regulation Is Satisfied } & \text { Source } \\ \text { IF } & \text { OSHA and Local Safety Regulations } & \\ & \text { Are Satisfied } & \text { User } \\ \text { AND } & \text { Environmental Hazards Are Acceptable } & \text { User } \\ \text { AND } & \text { Pile Driving Level of Noise Is Acceptable } & \text { User, Context } \\ \text { AND } & \text { Hammer Vibrations Are Acceptable } & \text { Context, User }\end{array}$

THEN Regulatory Conditions Are Satisfied

\section{\{Control Clauses if Accepted\}}

AND/OR Evaluate Another GWSS Alternative

AND/OR Rank Preliminary Feasible Alternatives

AND/OR Check Detailed_Feasibility Regulations

\{Control Clauses if Not_Acceptable\}
AND
Eliminate SSP
AND
Go to Next GWSS Alternative

The first premise ensures that safety regulations relevant to working conditions for labor are satisfied. This means that proper labor allocation, labor protection, and so forth, have to be met for the major operations (excavation, pile driving, muck removal, etc) of a proposed method, where some methods require more consideration than others. The user has to ensure the validity of this clause since there are numerous provisions to meet. The second premise verifies acceptance for the environmental hazards of a method. For example, if diesel material disposal is not possible in some areas, the diesel hammers class will be dismissed. This condition must be verified by the user. 
The third premise sets a lower bound for an accepted noise emanating from pile driving, e.g. noise from construction is being severely limited with an objective of not more than $85 \mathrm{~dB}$ at $50 \mathrm{ft}$ created by compressors for hammers and vibros (Hunt 1979). This limitation could be checked by the derived context where properties of pile driver components are retrieved from its data base and confirmed by the system. The user may exercise his judgment as to whether further restriction are warranted. The fourth premise ensures that vibrations emanating from pile driving do not destabilize surrounding structures, where, in some cases, surrounding structures are braced or other precautions are warranted. Similar to the previous premise, user or derived context could be used to approve this condition.

once this rule category is satisfied, and by implication, others as well, then a GWSS method alternative is considered to be preliminarily feasible. In this example, steel sheet pile, is regarded as preliminarily feasible. After an SSP is successfully selected, another GWS alternative in the list of the available alternatives will be tested for preliminary feasibility by repeating the same cycle shown above.

The foregoing control structure may allow solution synthesis to continue even if a high level condition failed 
(say regulatory condition), or was violated, within limits. The system may allow the user to further pursue and explore partial solutions for the components that remain feasible.

Therefore, a tentative list of preliminarily feasible alternatives is available for further synthesis at the detailed level.

\subsubsection{Detailed Feasibility Level}

The detailed feasibility level contains detailed design and analysis knowledge which is organized into several knowledge bases as shown in figure 4.6. The objectives of this level are to confirm the feasibility of alternatives produced at the preliminary feasibility stage, and to complete the frame description of each method which survives all feasibility checks. The control strategy guides this process beginning with the design component knowledge base (KB 3,4, and 5). At first, technical feasibility for an attribute is sought through simplified analysis and design procedures $(\mathrm{KB}-3)$. For instance, if the available sheet piles, represented by a data base, do not contain the required section, then a message is sent to the user advising of the technical infeasibility of the attribute. Control is then passed to the next preliminary GWSS alternative.

Assuming the steel sheet pile (propped sheet piles) design element was feasibly sized, control is passed to the 
pile driver selection and sizing knowledge base (KB-4). Based on the soil profile and conditions, and specified design element of the steel sheet pile attributes, the system attempts to pick the most suitable and productive hammer type and size from a hammer data base. The hammer selection must satisfy the technical feasibility conditions. on the other hand, if the hammer type and size is selected first, because of availability, this may dictate the size of the sheet pile. Thus, steel sheet and hammer sizing could be reversed. For CMSA, the former knowledge processing approach design element sizing to hammer sizing is adopted.

Included in the technical feasibility test for the hammer type and sizing, is the pile driving strategy (KB-5), i.e., drive piles in singles or in pairs. (Other strategies may include other wave patterns). The choice of either strategy is dependant on the soil conditions, pile driving energy, and so forth. After a steel sheet pile, hammer, and strategy are specified, a prediction module is applied to predict the performance measures (time and cost) for the candidate alternative $(\mathrm{KB}-6)$. After the cost estimate for the alternative is attained, control is passed to the risk assessment routine (KB-2), which uses the risk assessment framework described in section 4.4. It is used to determine whether or not the risks associated with the GWSS alternative, when priced out, exceeds some maximum threshold 
value. If they do, the alternative is deemed to be infeasible and the control strategy moves to the next candidate.

If the alternative's risk costs are acceptable, then it passes control to the diagnosis or analysis component (KB7). If the method synthesis (technical feasibility) is not satisfied, then $\mathrm{KB}-7$ recommends a change in either construction strategy, hammer type or size, or steel sheet pile type, size, and grade. Currently, only the first is automated, with the alternatives being to drive piles in singles or in pairs. If the recommendations still yield an infeasible SSP GWS alternative, then the alternative is declared to be infeasible. If it is technically feasible, then production rate and unit cost are determined using the prediction module of $\mathrm{KB}-6$.

At the end, an evaluation criterion will be chosen to rate the feasible SSP alternative in order to rank it with other successful candidates. Section 4.5 elaborates on several criteria schemes.

other issues relevant to this section concern what to do when a solution fails within the system -- what kind of remedy to explore, what attributes values have to be remembered, where to track back to, and when the system stops and declares an alternative is not feasible within the system. 
In the discussion that follows, a step-by-step approach is presented, along with illustrative rules to demonstrate the approach just outlined. First, we examine the design element synthesis.

\section{Design Element synthesis (KB-3)}

Attention is directed at sizing the design element (in this case the steel sheet piling) to satisfy the technical feasibility requirement. For this example, technical feasibility means that given default attributes for a support system, piles can be sized so that stress and deflection criteria (not implemented) are satisfied. A three part process is adopted. First, a rule-based approach is used to determine pressures and moments. Second, a search is made for a sheet pile that satisfies the allowable stress criteria. Third, if a pile can't be found, the retaining strategy is altered (spacing of struts and/or wales is involved) and control is passed back to the second step, with iterations occurring until either a feasible steel sheet pile design is confirmed or no feasible solution exits. This third step has not been implemented in the prototype. Example rules for the first two parts of this process are now described.

\subsection{Pressure and Moment Calculations (KB-3-1)}

Rules are used to retrieve the required properties corresponding to the soil strata scenario (context 
information), to assign the default horizontal and vertical spacing for the retaining spacing (struts spacing horizontally $12 \mathrm{ft}$ and vertically $15 \mathrm{ft}$, and to perform the calculations of pressure and moments. Details of the calculations are given in Appendix $\mathrm{A}$.

An example of the rule format follows.

Rule: Compute Earth Pressure and Moments

for the two Soil Layers Scenario

Source

IF Soil_Layers.Number is Two User

AND Soil_layer_1 is Loose_Sand User

AND Soil_Layer_2 is Stiff_Clay User

THEN Maximum_Lateral_Pressure $=($ Loose_Sand.Unit_weight $*$

Loose_Sand.Depth * (K(a) for LS) + Stiff_Clay Unit Weight *

Stiff_Clay_Layer.Depth) $*$ (K(a) for SC)

AND Moments for Steel Sheet Piles = Maximum_lateral_Pressure *

L (vertical spacing) $2 / 8$

\{Control Strategy\}

AND Specify the Steel Sheet Pile Section

The first three premises query the user for the soil profile. The system retrieves relevant soil properties to compute the lateral earth pressure -- unit weight, $\mathrm{K}(\mathrm{a})$, etc. In the concluding part, pressure and moments are computed (See Appendix A), and control is passed to specify the sheet pile.

\section{2 selecting and sizing Design Elements [KB-3-2]}

Moment information is passed from the first step to the second, and combined with an allowable stress condition to determine the section modulus required. If the modulus required exceeds the maximum size available in the data 
base, the current design is technically infeasible. Feasibility may be achieved by modifying the spacing of the retaining system.

The KB-3 design component contains mainly factual rules applied for sheet piles, soldier piles, lagging, wales, and struts. It currently uses a single criterion based on strength. Other design criteria such as deflection, allowable settlement, and so forth, have not been treated. A typical rule employed is:

$\begin{array}{lll}\text { RULE } & \text { Select a Steel Sheet Pile } & \text { Source } \\ \text { IF } & \text { SSP.Section_Modulus = Maximum_Moments } / & \\ & \left(\mathrm{Fb}^{*} \text { Fs }\right) & \text { Context (step 1) } \\ \text { AND } & 38.3 \text { in } \bumpeq<\text { Steel_Pile_Section_Modules }<= & \text { Context } \\ & 46.8 \text { in }{ }^{\wedge} & \\ \text { THEN } & \text { SSP.designation Is PZ_27 } & \\ \text { AND } & \text { Retrieve PZ_27 Properties Database } \\ \text { AND } & \text { Calculate the Quantity Take-Off for Sheet Piles }\end{array}$

\section{\{Control Strategy\}}

AND Select a Hammer Class

This rule is internal, where derived context from the previous rule is used to specify a SSP of PZ_27. Control is passed to hammer selection next.

KB-3-3, although not implemented, would permit changing the retaining system in an attempt to find a feasible steel sheet pile size. 


\section{Specifying A Construction Resource (Resource Level)}

\section{1 selecting a Hammer $(\mathrm{KB}-4-1)$}

The main types of hammers include:

1. Drop hammers;

2. Steam hammers (Single and Double Acting),

3. Air hammers (Single and Double Acting);

4. Diesel Hammers (Single, Double, and Differential Acting hammers;

5. Hydraulic hammers;

6. Vibratory Hammers; and

7. others.

Selecting the most suitable pile hammer for a given project involves the consideration of several factors, such as size and piles types, number of piles, characteristics of the soil, location of the project, topography of the site, type of rig available, and the types and sizes of hammer owned by contractor.

A pile driving contractor usually is concerned with selecting the hammer that will drive the piles for a project at the lowest cost within the required production rate. Broad brush rules found in the literature are similar to the one shown in table 4.6, which recommend the hammer most suitable for different homogeneous soil classifications. Such a table is convenient for a one_soil_Layer representation; however, the selection process is more complicated when two or more soil layers are present. The 
selection depends on the ordering of the soil layers, the depth of each layer, and the SPT profile. For example, if the top layer is soft soil and the lower layer is dense soil, a vibratory, light impact hammer is used to drive them to the dense layer, and then another, heavier hammer is utilized to drive them to refusal.

\begin{tabular}{|c|c|c|c|c|c|c|}
\hline \multicolumn{7}{|c|}{ BANDS (NON-COHESNE SOLL) } \\
\hline & Wood & \begin{tabular}{|l|} 
Pipe \\
Open
\end{tabular} & $\begin{array}{l}\text { Plpo } \\
\text { closed }\end{array}$ & $\mid \begin{array}{c}\mathrm{H}- \\
\text { Deam }\end{array}$ & $\begin{array}{l}\text { Shent } \\
\text { plis }\end{array}$ & $\begin{array}{l}\text { Cone } \\
\text { orete }\end{array}$ \\
\hline Very Loose & DA & $\begin{array}{c}\mathrm{V}(\mathrm{NB}) \\
\mathrm{bA}^{\prime}\end{array}$ & $\begin{array}{c}\mathrm{Y}(\mathrm{NB}) \\
\mathrm{bA}\end{array}$ & $\begin{array}{c}\mathrm{V}(\mathrm{N}) \\
\mathrm{DA}\end{array}$ & $\begin{array}{c}\text { V(NB) } \\
\mathrm{bA}^{\prime}\end{array}$ & DA \\
\hline Loose & DA & $\begin{array}{c}\text { V(NB) } \\
\text { DA }\end{array}$ & DA & $\begin{array}{l}\text { V(NB) } \\
\text { DA }\end{array}$ & V(NB) & DA \\
\hline Medium & SA & $\begin{array}{c}\mathrm{Y}(\mathrm{NB}) \\
\mathrm{bA}\end{array}$ & DA & $\begin{array}{c}\mathrm{V}(\mathrm{NB}) \\
\mathrm{DA}\end{array}$ & $\begin{array}{c}\mathrm{V}(\mathrm{NB}) \\
\mathrm{DA}\end{array}$ & SA \\
\hline Denes & SA & $\begin{array}{c}(N B) \\
D A\end{array}$ & SA & \begin{tabular}{|c|}
$\mathrm{V}(\mathrm{NB})$ \\
$\mathrm{BA}$
\end{tabular} & $\begin{array}{c}\mathrm{V}(\mathrm{NB}) \\
\mathrm{bA}^{\prime}\end{array}$ & SA \\
\hline Very Denses & SA & SA & SA & 8A & $\mathrm{V}(\mathrm{NB})$ & SA \\
\hline
\end{tabular}

(a)

\begin{tabular}{|c|c|c|c|c|c|c|}
\hline \multicolumn{7}{|c|}{ CLAYB (COHEBNE 8OLL) } \\
\hline & Wood & $\begin{array}{l}\text { Pipe } \\
\text { Open }\end{array}$ & $\begin{array}{l}\text { Plpo } \\
\text { Closex }\end{array}$ & Beam & \begin{tabular}{|l|} 
8heed \\
Plie
\end{tabular} & $\begin{array}{l}\text { Cono } \\
\text { erete }\end{array}$ \\
\hline Very son & DA & $\begin{array}{c}\text { V(NB) } \\
b_{A}\end{array}$ & DA & $\begin{array}{c}\mathrm{V}(\mathrm{NB}) \\
\mathrm{DA}^{\prime}\end{array}$ & $\mathbf{v}$ & sa \\
\hline Medlum & DA & $\begin{array}{c}\text { V(NB) } \\
\text { DA }\end{array}$ & SA & $\begin{array}{l}\mathrm{V}(\mathrm{NB}) \\
\mathrm{DA}\end{array}$ & $\begin{array}{c}Y \\
\text { DA }\end{array}$ & sa \\
\hline 841 & SA & DA & SA & DA & DA & SA \\
\hline Vory suin & SA & SA & SA & SA & SA & SA \\
\hline Hard & SA & SA & SA & SA & SA & SA \\
\hline
\end{tabular}

(b)

DA - Double Aoting (Dinel or Ar/scient)

8A. Binglo Acting (Diceal or Arrovarm) V. Vibritory

NB - No Bearing Formule Alequined

Table 4.6 Hammers for Different soils [from Barber 1987] 
A typical rule format for the single soil layer case is:

$\begin{array}{llr}\text { RULE } & \text { Select A Single Acting Air Hammer } & \text { Source } \\ \text { IF } & \text { Soil_Layers.Number = One Soil Layęr } & \text { User } \\ \text { AND } & \text { Type of Soil Profile = Cohesive Soil } & \text { User, Context } \\ \text { THEN } & \text { Select An Impact Hammer } & \text { Context (Experiential) } \\ \text { AND } & \text { Choose A Single Acting Air Hammer } & \\ \text { OR } & \text { Choose Double Acting Air Hammer } & \end{array}$

\section{Control Strategy}

AND Specify a Hammer Size

The first and second premises query the user for the soil profile. The latter inquires about the cohesiveness of the layer although the system is already aware of the soil layer properties. The reason for this is to allow the user to use his judgement in determining this quality since this clause, if true, excludes the vibratory class from consideration and thus focuses on the impact hammer class.

For two soil layers, there could be several feasible hammer alternatives -- e.g. use a single hammer (impact or vibratory), use a combination of hammer types, or a range of sizes of the same hammer (use a lighter one to drive the top, soft layers and the heavier one to drive piles to their refusal depth).

${ }^{3}$ Vibratory pile driver use is not recommended for a soil profile with a sizable cohesive layer (e.g. clay). Therefore, this premise eliminates the vibratory pile driver subset and examines impact hammers only. 
A typical rule format for the two soil layers case is:

RULE

IF

AND

AND

THEN

AND
Select A Single Acting Air Hammer

Top_Soil_Layer is Loose_Sand Lower Söil_Layer Is Stiff Clay Stiff_Clay_Layer.Depth $>15 \mathrm{ft}$ Choose A Single Acting Air Hammer Size the Hammer
Source

Context

Context

Context

\section{\{Control Strategy\}}

AND Specify a Hammer Size

The above rule checks the cohesive soil layer thickness to a threshold which excludes vibratory hammers. If the clay depth to the sand layer ratio is very high then a vibratory pile driver could be favorable. Thus, for this rule, the impact hammer, single acting air hammer, is selected with reference to table 4.5 .

\section{2 sizing The Hammer $[\mathrm{KB}-4-2]$}

The sizing process starts by selecting the highest hammer theoretical energy. This is consistent with the "greedy" algorithm, described previously, in which maximum production rates and reserve capacity are sought. Two additional conditions are examined however. First, damage to the sheet pile must be avoided. Second, the hammer must be capable of driving the pile to refusal depth ${ }^{4}$. If either of these ${ }^{4}$ Refusal is the depth to which piles have to be driven, to attain their designed resistance strength through skin and end bearing. For non-displacement piles (see figure 4.7), sheet piles and soldier piles, their depth extends below the tunnel bottom (excavation depth) by 5 ft to $15 \mathrm{ft}$ (Winterkorn and Fang 1975). 
conditions cannot be met, then either the hammer energy is decreased and/or the sheet pile size is increased. If no satisfactory solution can be found, the GWSS alternative of SSP is considered to be infeasible.

A rule format for determining Hammer size according to SSP size, using empirical knowledge, is shown below.

$\begin{array}{lll}\text { RULE } & \text { Size the Hammer } & \text { Source } \\ \text { IF } & \text { SSP_Cross_Section_Area is Ap } & \text { Context (SSP Database) } \\ \text { AND } & \text { Hammer_Type Is Single_Acting_Air_Hammer } & \text { Context } \\ \text { THEN } & \begin{array}{l}\text { Hammer_Size (Hammer_Rated_Energy) } \\ =<\quad 3,000^{*} \text { Ap (lb-ft) }\end{array} & \text { Context } \\ & \begin{array}{l}\text { Single_Acting_Air_Hammer.Size }> \\ \text { AND }\end{array} & \text { (DAAH_Database), Context } \\ & \end{array}$

\section{\{Control Strategy\}}

AND Do Pile_Driving_Strategy

The above rule, based on contractors' experience, determines the maximum magnitude of the hammer energy by multiplying $3,000 \mathrm{lb} / \mathrm{in}^{\wedge} 2$ by SSP_Cross_Section_Area to prevent pile damage. After executing this rule successfully, control is passed to selecting the pile driving strategy.

\section{Pile Driving strategy [KB-5]}

Soil conditions and hammer power dictate the pile driving strategy in terms of driving in singles or pairs. Secondary factors relevant to this strategy are SSP size and length of 
pile segment. To prevent buckling, the maximum allowable driving depth with respect to adjacent piles is $<=13 \mathrm{ft}$.

As a greedy approach is favoured, and as other contractors have suggested that trying to drive piles in pairs is a preferred approach, the pile driving strategy In_Pairs is selected first. Driving conditions are restricted to two states: soft and hard driving conditions. They are inferred from the soil conditions using simplified rules. For the former, soft driving conditions, the driving strategy is In_Pairs; while the latter driving strategy is In_singles. Other pertinent factors such as hammer type and size, type and grade of sheet piles, number of pile drivers and complexity of the project were not considered explicitly.

The following rules exemplify the conditions for pile driving In_singles and In_Pairs. Pile driving In_singles rule (experiential) is:

RULE

IF

AND

AND

THEN

AND

\section{Drive in Singles}

Pile_Driving.Conditions Are Hard

Refusal.depth $>30 \mathrm{ft}$

Hammer.size $<22,000 \mathrm{Ib}$-ft

Drive Piles In_Singles

Do Performance Measures

\section{\{Control Strategy\}}

Source

Context, User

Context

Context

AND 
Pile driving In_Pairs rule (experiential) is:

$\begin{array}{lll}\text { RULE } & \text { Drive Piles in Pairs } & \text { Source } \\ \text { IF } & \text { Pile_Driving.Conditions Are Soft } & \text { Context, User } \\ \text { AND } & \text { Refusal.depth }<90 \mathrm{ft} & \text { Context } \\ \text { AND } & \text { Hammer.size }>10,000 \mathrm{Ib}-\mathrm{ft} & \text { Context } \\ \text { THEN } & \text { Drive Piles In Pairs } & \end{array}$

\section{\{Control Strategy\}}

AND Do Performance_Measures

\section{Predict $(\mathrm{KB}-6)$}

The steps involved are:

1. Determine production rate and cost.

2. During pile driving production analysis, a pile damage check is involved (blow counts)

If

blow count exceeded, then backtrack and change one or more choices

otherwise determine production + cost. If not acceptable, then..

In choosing between pile driving In_Pairs or In_singles, consideration must be given to both set up time and driving time.

\section{Production Time and cost Performance}

Given a feasible SSP size, hammer type and size, and driving strategy, then it remains to determine time and cost performance, and perform other checks, such as regulatory considerations on noise level ${ }^{5}$.

${ }^{5}$ Safety and regulation factors are not implemented at this level. The noise level is implicitly satisfied as affirmed by the preliminary regulation feasibility check. 
Using a hammer dynamic energy (modified Engineering News formula ${ }^{6}$ ) production routine written in c (Drive.c), control is passed to this routine for purposes of computing a pile driving production rate excluding fixed set up time. Output information is passed back to the control strategy which in turns interprets the routine results. A constraint is included dealing with the maximum number of blows per foot, beyond which pile damage is likely. In the event that this blow count is reached, the routine stops summing up the incremental production time and returns a message to CMSA indicating this violation and where it happened -- i.e. the depth of pile where it interrupted driving.

The hammer blow count is empirical and can vary with different soil/pile/hammer where technical feasibility is monitored by observation. For CMSA, the system takes action based on a theoretical blow count from the model derived in Appendix B.

${ }^{6}$ This formula, among numerous of hammer energy formulas, is applied only to some types of impact hammers. For vibratory pile drivers, rule of thumbs are utilized to estimate their productivity. 
The rule format for running the "Drive.c" routine is:

$\begin{array}{lll}\text { RULE } & \text { Do Performance Measures } & \text { Source } \\ \text { IF } & \text { Selected_SSP_Type Is PZ_27 } & \text { Context } \\ \text { AND } & \text { Selected_Hammer = Single_Acting_Air_Hammer (SAAH) } & \text { Context } \\ \text { AND } & \text { SAAH.Rated_Delivered_Energy =15000 Ib-ft } & \text { Context } \\ \text { AND } & \text { Pile_Driving_Conditions Is Soft } & \text { Context } \\ \text { AND } & \text { Piles_Driving_Strategy Is "In_Pairs" } & \text { Context }\end{array}$

\section{\{Control Structure if Accepted\}}

THEN Compute Production_Rate 7

AND Do SSP.Risk_Assessment

\section{(Control Structure if Unaccepted\}}

ELSE Analyze Technical_Feasibility ${ }^{8}$

The above rule pools specified method attributes -- sheet pile, hammer and pile driving strategy, and sends them as input parameters to the Drive.c routine.

Figure 4.10 shows input parameters for the Drive.c routine passed by CMSA via an input text file which then is processed by the routine. After executing the numerical routine, output parameters are passed back to the control strategy for interpretation and further manipulation.

output variables include incremental and cumulative values for each of skin friction, end bearing soil

${ }^{7}$ Blow count satisfaction is implicit in this clause. If the threshold blow count per foot is violated, then control is passed to the technical feasibility analysis to investigate a remedy.

${ }^{8}$ Technical feasibility at this stage refers to whether a method attributes combination (SSP + Pile Driver + Construction strategy) achieves its goals of production, cost, damage free driving, and so forth. 
resistance, blow count, and production progress (relationships and runs are detailed in Appendix B) .

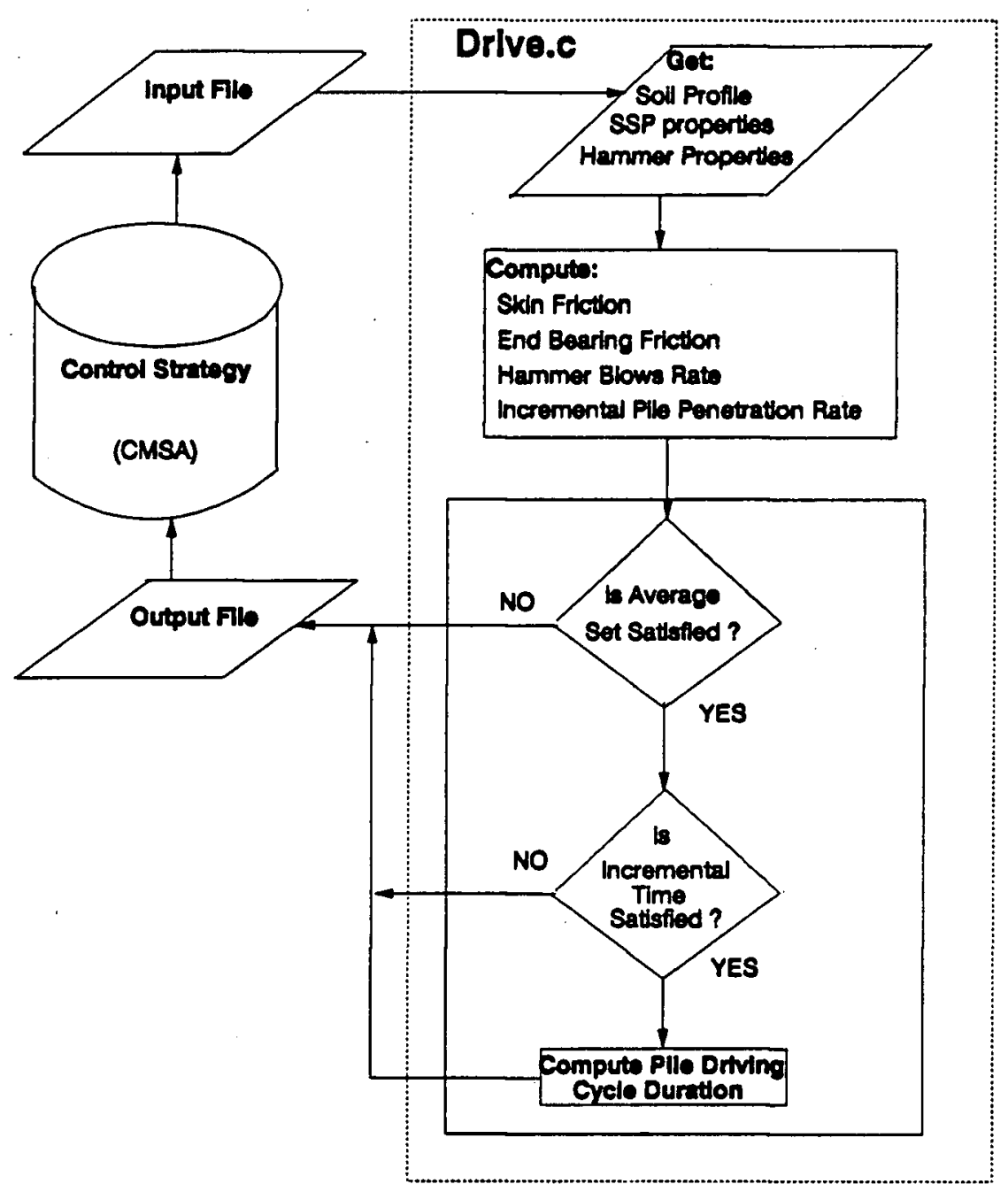

Figure 4.10 Drive.c Routine Interface with CMsA

A criterion of maximum acceptable hammer blow count of 150 blows/ft (a bench-mark from field engineers to interrupt if refusal is reached) is set as the threshold. If the blow count exceeds this limit, then the Drive.c routine stops 
computation, flags the depth where it happened and sends a message to the control strategy which in turn interprets that the method combination (technical feasibility) is not feasible, or else, it is feasible and the production rate is passed by the routine to the control strategy.

After the SSP alternative passed this test successfully, the quantity take-off and cost estimate computation for the whole project follow. Once detailed method cost is known, a risk assessment is ensued subsequently.

\section{Risk Assessment (KB-2)}

The risk factor has a significant impact on the choice of construction methods in general and on shoring method selection in particular. The considerable emphasis placed on an informal risk assessment, particularly with respect to the likelihood of catastrophic risk, was highlighted in an interview with a seasoned construction engineer (see Appendix C) .

Based on discussions with construction personnel, a review of the literature and an analysis of the amount of data likely to be available when making decisions about construction methods, a simplified analytical CMSA risk assessment framework was developed as described in section 4.4. What is important is the role of risk in the control strategy. If the potential for catastrophic or unacceptable 
risks are high, then a method will be dropped, not withstanding its appeal because of time or unit cost performance.

As described in section 4.4 , the risk model involves three states of nature of geological conditions, given a design alternative. The three states correspond to better than, equal to, and worse than expected conditions. Several cost categories describe the outcome for each state of nature. An example rule for the case when the state of nature is As_Expected, is as follows:

RULE Compute Risk for "As-Expected" State of Nature Source IF GWSS = Steel_Sheet_Pile (SSP) Preliminary Feasibility AND Soil_Conditions Is As_Expected Context
AND As_Expected_Conditions.Likelihood $=\mathbf{P p}$
User

AND Consequence Costs $(D p)=$ Sum $(D p(i))$

Context

THEN Compute As_Expected Risk_Cost_Component

AND As_Expected_Conditions.Risk_Component $=\left(\mathrm{Pp}^{*} \mathrm{Dp}\right)$

\section{Control Strategy}

AND Compute Other Risk Component Costs

The first premise identifies the candidate in order to evoke risk relevant slots from the preliminary level. The second premise affirms the state of nature by context. The third premise queries the user about the individual cost item estimates $(D p(i))$, while context adds them up. In the conclusion, the As_Expected risk element will be evaluated as the product of its likelihood by the sum of its cost 
items. Next, control is passed to compute other states of nature risk components -- i.e. better than expected and worse than expected.

Next, we examine the SSP method analysis component.

\section{Analyze (KB-7)}

The following control strategy rule examples are used to direct the search for changes to the construction method in order to achieve feasibility.

RULE (1) Change Pile Driving Strategy from "In_Pairs" to "In_Singles" Source IF Pile_Driving_Strategy Is "In_Pairs",

AND Technical_Feasibility.State is False ${ }^{9}$ Context, User (blow count $<=$ threshold) Context

\section{\{Control Strategy\}}

THEN Change Pile_Driving_Strategy to In_Singles

AND Do Performance Measures

RULE (2) Increase Hammer Energy Source

IF

Pile_Driving_Strategy Is In_Singles

Technical_Feasibility Is False

Context

AND

\section{\{Control Strategy\}}

THEN Increase the Hammer Delivered Energy

AND Do Performance Measures

${ }^{9}$ Appendix B contains the logic and relevant dynamic formulas derivation for the technical feasibility test under pile/hammer/soil scenarios. 
RULE (3)

IF

AND

Driving_Strategy Is In Singles

Hammer/Pile Are Not_Compatible
Context

Context

\section{\{Control Strategy\}}

THEN Change SSP_Alternative to SPL_Alternative

AND Do Design SPL_Alternative

\subsection{CMsA Risk Component Development and Evaluation}

Interviews and discussions with contractors' personnel regarding the decision making process dealing with methods selection have highlighted their concern, early on in the process, with risks. Particular emphasis is placed on the potential for large/catastrophic risks which often accompany underground work, work in water, etc. A method which is more likely to be subject to such risks tends to be shunned even if there are significant cost/time benefits associated with it. Contractors tend to seek a solution that actively, as opposed to passively, controls risk (e.g. H piles and lagging with struts rather than shotcrete).

Based on the above, the use of a risk criterion to screen alternatives early on is important. The risk assessment framework should be simple to use and not overly data intensive. For example, a complex set of states of nature may realistically portray the operating environment for a method, e.g. see figure 4.11 .

The effort and data required to specify each risk type and corresponding states simply are not available. So risk assessment for Cut-and-Cover tunnelling alternatives for 
this thesis, must be simplified to treat only those conditions that could lead to unacceptable risks.

In this section, the context of GWS is used to illustrate the approach. Two risk categories are considered:

\section{Figure 4.11 states of Nature for Methods selection}

1. Normal Risks: Normal risks deals with those site conditions (access, weather, ground, management, etc.) that influence productivity and other variables, thus creating uncertainty in time and cost estimates.

2. Large/Catastrophic Risks: These are treated explicitly in the decision making process through a simplified decision tree shown in figure 4.12 . The basis of this decision tree 
this thesis, must be simplified to treat only those conditions that could lead to unacceptable risks.

In this section, the context of GWS is used to illustrate the approach. Two risk categories are considered:

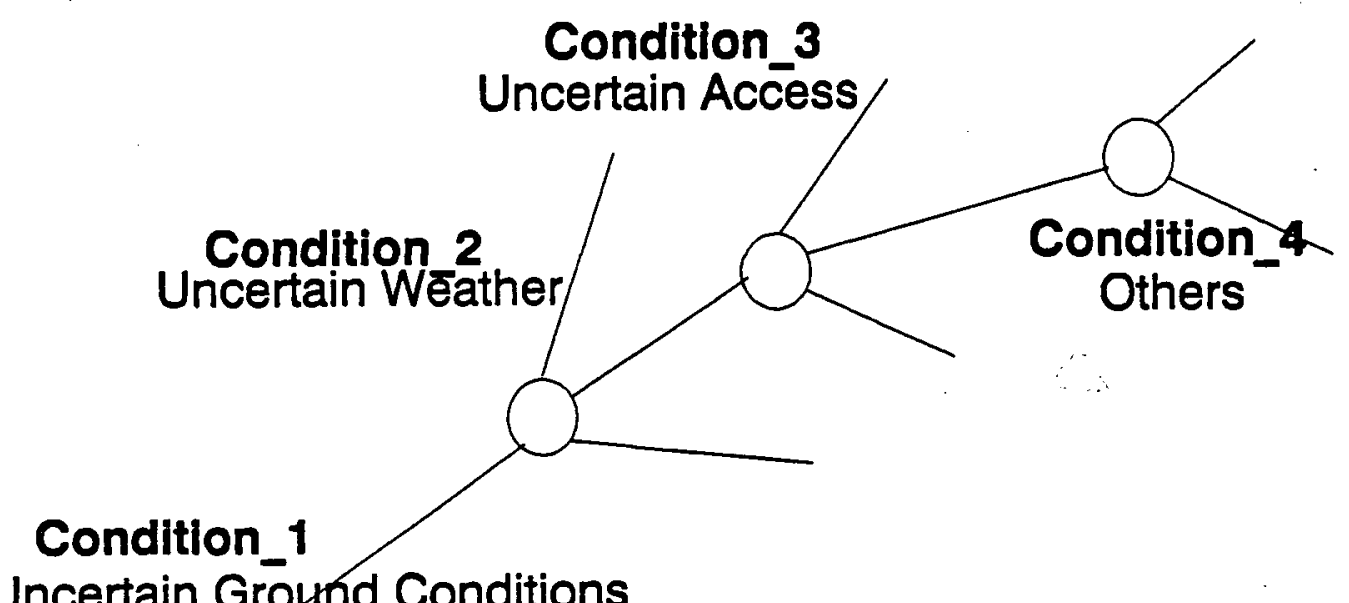

Uncertain Ground Conditions

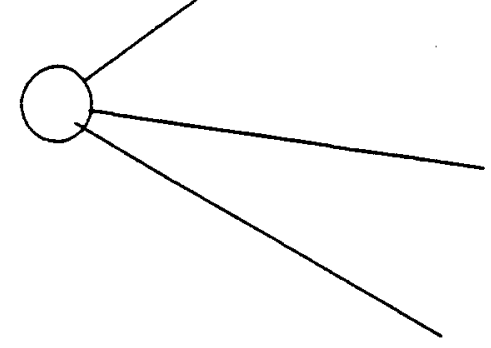

Figure 4.11 states of Nature for Methods selection

1. Normal Risks: Normal risks deals with those site conditions (access, weather, ground, management, etc.) that influence productivity and other variables, thus creating uncertainty in time and cost estimates.

2. Large/Catastrophic Risks: These are treated explicitly in the decision making process through a simplified decision tree shown in figure 4.12. The basis of this decision tree 
is as follows. First, since we are using risk to preserve alternatives, we examine alternatives indirectly. Second, three basic state of nature are treated:

1. conditions better than expected;

2. conditions as expected; and

3. conditions worse than expected.

Conditions here refers to that condition most likely to lead to unacceptably large or catastrophic risks. Then, for each state of nature treat three more conditions -- no failure, minor failure, major failure; or no damage, element damage, system damage, where:

1. minor failure refers to partial damages for the wall structures and/or the surroundings; and

2. major failure refers to GWSS collapse, or retaining system collapse, and/or other major surrounding damages.

The user is required to assign probabilities to each branch in the decision tree. At the end of each path is a vector of incremental costs (positive/negative). This vector of costs is:

\author{
Labor \\ Equipment \\ Materials \\ Loss of Life \\ Loss of Reputation \\ Subsurface Subsidence \\ Season Loss
}

The user is asked to estimate the costs associated with this vector as a fraction or percentage of total estimate, 
given the states of nature. This corresponds to the way contractors estimate.

In terms of computation, the incremental costs are summed, and then discounted by the probabilities. If risks are deemed to be unacceptable, the user is required to assign an infinite cost to a cost category. This effectively eliminates the alternative. Then,

Total cost of alternative

$=$ base cost + expected value of incremental costs

For further development of the expert system, the challenge is to identify, for each method alternative, the governing risk considerations (e.g. ground condition variable, flood potential, etc.) We also need to allow the facility for the user to describe and record the thought process that leads to the specification of the decision tree. 
Shoring Alternatives

Event Chances
Consquences Costs

(Outcomes)

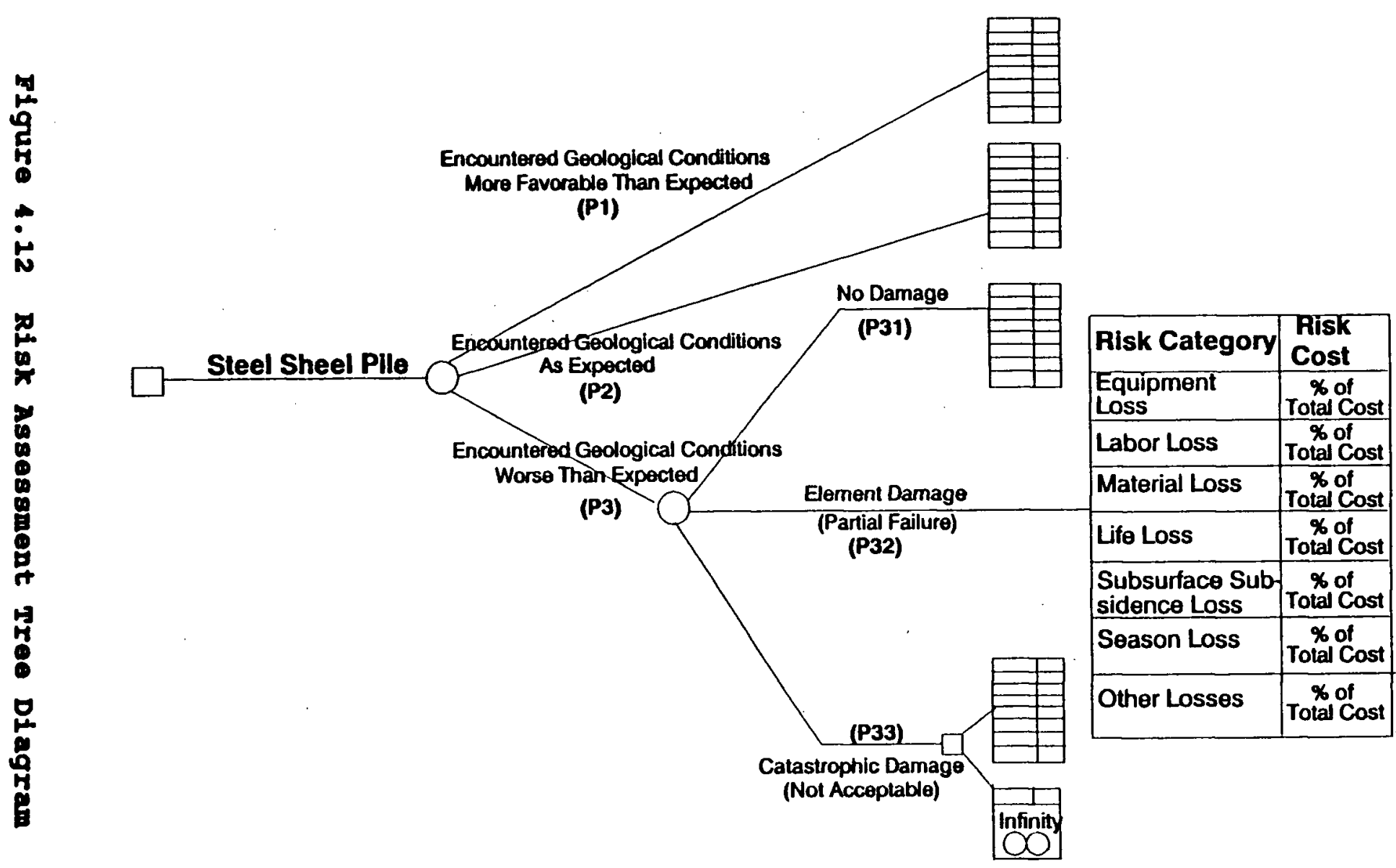




\section{CMSA Implementation}

\subsection{Introduction}

The primary objective of this chapter is to explore the issues involved in the implementation of a prototype CMSA system. The first part covers an overview of NExpert object, an expert system used to implement CMSA. The objective is to familiarize the reader with the NExpert environment: knowledge constructs, syntax, operators, inference mechanism, and so forth.

The second part covers selected details of the CMSA prototype development. The heuristic problem solution paradigm (figure 4.6) consists of suggest, Design, Predict, and Analyze operators. The first operator, suggest, maps the preliminary knowledge that suggests a preliminary feasible GWSS alternative for further detailing by the low level feasibility part. The other three operators correspond to the low level feasibility component: Design specifies the design element, construction resources, and construction strategy; Predict applies the hammer dynamic formula to predict its performance; and Analyze diagnoses the synthesized method to test its feasibility. If the assembled method meets its goals, then it accepts the GWSS alternative (SSP). If it does not meet its goal, then it suggests recommendations for re-designing the method. 
The construction method attributes of the design elements, and construction resources are represented as frames. Rules are used to represent other specific knowledge and control strategy. These include engineering design knowledge for structural members (sheet piles), resource selection (pile drivers selection), construction strategy (pile driving pattern), and construction process model evaluation (dynamic formulas), analyzing the method synthesis (technical feasibility), and ranking the GWSS alternatives. Examples of frames, rules, and databases written in NExpert object will be provided.

Rúles of thumb and algorithms as presented in the literature review in chapters 2,3 , and 4 and in the appendices were extracted from texts, journals, and interviews. Knowledge acquisition for methods selection and analysis is the domain of a number of experts, engineers, superintendents, foremen, and others. Each contributes to the method selection process at different times, and to varying degrees. Knowledge acquisition from site and office experts from one project was undertaken. Appendix $C$ describes a site visit and provides the results of two interviews. Such interviews contributed to improving and validating the problem solving knowledge base. 


\subsection{NExpert Object Overview}

An AI toolkit is ideal for problems that need a mix of specialized tools. It is desirable that such a toolkit should offer a hybrid rule system, object oriented programming, and access to a general purpose language. Additionally, the toolkit should interface with conventional software such as databases, spreadsheets, graphics packages, and word processors. Neuron Data's NExpert object exhibits several of these features.

NExpert is a powerful, hybrid, rule and object based expert system shell that speeds up the prototyping process for expert systems for non-programmers. It is mainly a rule based system fitted with object oriented features, such as firing a routine from a premise if certain conditions have changed. The version used by the author suffered from a lack of good documentation and examples to explain and demonstrate all of NExpert's features.

\subsubsection{Major NExpert object Modules}

NExpert object consists of the following modules:

1. NExpert Development Package, is the core of the system. It contains pop-up windows for editing text, database, rules, objects, classes, etc; visual display for rules and objects networks; a reasoning kernel providing inheritance control, backward chaining, forward chaining, pattern 
matching calls to external routines, and others; and trace facilities (Transcript, Encyclopedia, Reports).

2. The callable Interface, is a library of $C$ routines and function kernels used to embed NExpert within a conventional programming language. It consists of $\mathrm{C}$ functions that make up the NExpert object Development and Runtime Environments. It can be used to establish communications between external application programs and NExpert applications by taking advantage of structures and functions that NExpert uses internally.

There are at least three ways to use the callable interface. NExpert functions can be called directly by external code as exported $C$ functions; external code can "trap" specific NExpert functions and declare their own replacement, or a standard message can be passed between other windows applications and NExpert using Microsoft's dynamic exchange protocol (a system for setting up standard messages). The callable interface includes $c$ functions to initialize, start, stop, and resume sessions; query or change knowledge structures; and change the list of hypotheses or agendas.

In order to install function handlers in NExpert, or embed NExpert within another application, there are additional software requirements such as the MS Windows 
Software Development Kit, version 2.03 or later, and a Microsoft C Compiler version 5.0 or later.

3. NExpert object Runtime, is a run time package that is used to run an application without access to the knowledge base (a development package stripped of its debugging mechanisms). The developer can define, in detail, how an application will run and design an interface that is visible to the client.

An overview of the NExpert development framework is shown in figure 5.1 .

4. Hardware and Software Requirements -- Minimum hardware and software requirements for NExpert object under MS/Windows are:

IBM PC or compatible with $640 \mathrm{~K}$ of conventional memory, plus

- 1 MByte of expanded memory on a 286 machine

- 1 MByte of expanded or extended memory on a 386 machine (2M if you are using Windows 386)

- Enhanced Graphics Adapter (EGA) or VGA. Color EGA requires a video board with at least $64 \mathrm{~K}$ of memory.

- 1.44 M floppy disk drive

- hard disk with at least 3 MByte available

- Microsoft Windows Runtime 286 or 386, version 2.03 or later.

- Mouse (compatible with MS-Windows: Bus or Serial, Microsoft, LogiMouse, Mouse systems, etc.), Parallel port. 
Recommended hardware and software in addition to the above:

- 2 additional MBytes of Extended memory. This allows you to store NExpert object in RAM drive, - 386-based machine (for development)

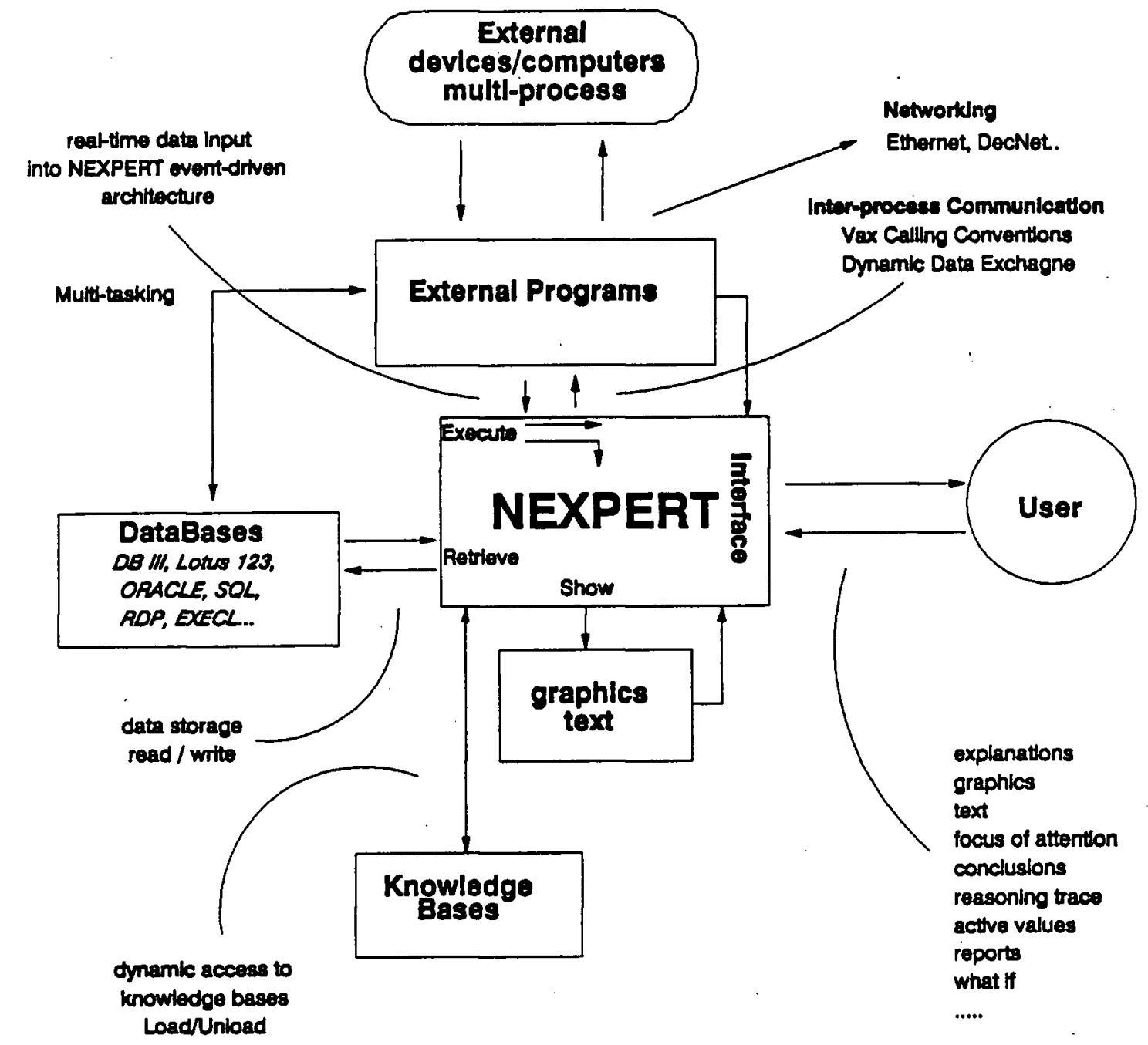

Figure 5.1 NExpert Object Open AI Environment Framework [from Neuron Data 1989] 


\subsubsection{NExpert Primitives -- Building Blocks and Operations}

The basic building blocks of NExpert object are described in this subsection.

\section{Rules}

Rules are the preferred way to process objects in either forward or backward chaining. In NExpert, rules are expressed in the following form (see figure 5.2 for NExpert Rule Syntax)

$\begin{array}{ll}\text { IF } & \begin{array}{l}\text { Condition1 } \\ \text { AND }\end{array} \\ \text { Condition2 } \\ \text { AND } & \text { ConditionM } \\ \text { THEN } & \text { Conclusion (Hypothesis) } \\ \text { AND } & \text { Action1 } \\ \text { AND } & \text { Action2 } \\ \text { AND } & \text { ActionM }\end{array}$

The left hand side (LHS) of a rule tests the value of a property for some object or class. Mixed qualifiers such as existential operator (test the condition: is there any instance ...) and universal operator (test the condition: are all instances ...) can be used to lend expressive power to the LHS conditions.

The LHS of the rule is composed of one or more antecedent (if) clauses which are called conditions. Operators and their purposes on this side include: 
Yes and No test Boolean Variables;

$[>,<,=,<>$,

to test an expression or variable against a numerical value;

Is and IsNot test string Type Data versus reserved Keywords ;

Name

Evaluate an expression and/or variable and assign it to another variable;

Reset

reset the value of an attribute to the original Unknown, as if the value has not been asked;

Equal and

Notequal

compare an expression to a slot;

show

used to show a text file or a graph during running an application;

Member and NotMember

test whether a particular object belongs to a given list of objects obtained by pattern matching or: verify that an object does not belong to a list:

Execute

call a subroutine written in a high level language such as $\mathrm{C}$ or FORTRAN;

Retrieve

allows the system to read a file of values stored on disk and/or to query a database.

A right hand side (RHS) of a rule has a conclusion or hypothesis and optional actions. The hypothesis defines the rule's major topic and, as such, can be used to control backward and forward chaining. If two rules share the same RHS hypothesis, then both become candidates in backward chaining on that hypothesis. A LHS condition in one rule can also be the RHS hypothesis in another. 


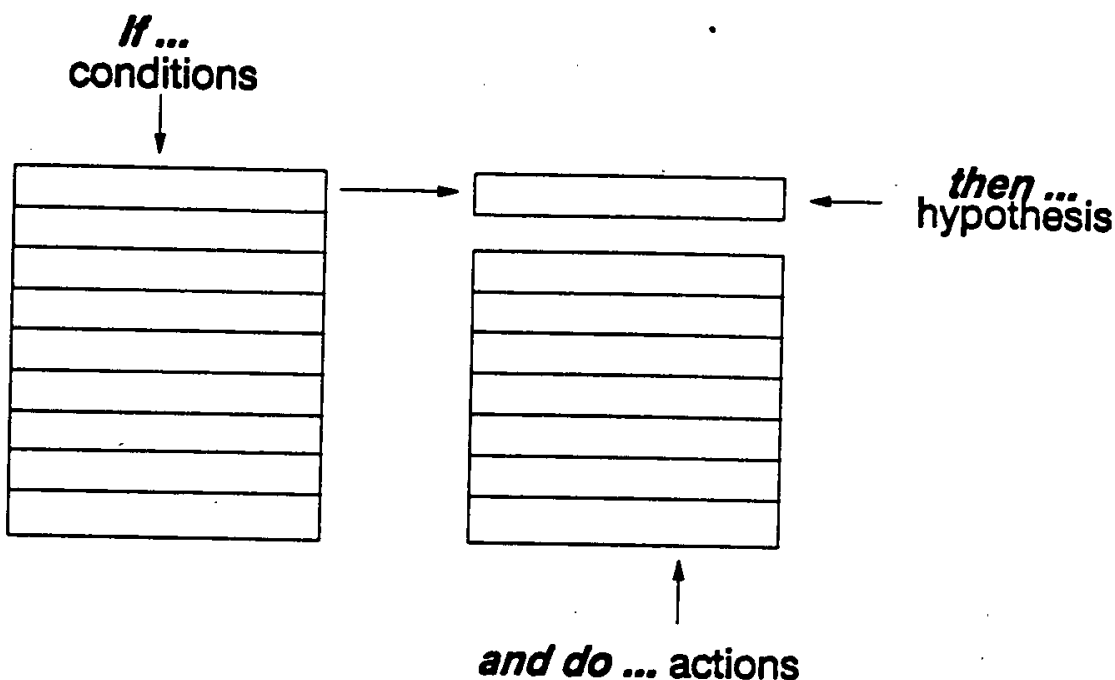

\section{Figure 5.2 NExpert Rule Construct [from Neuron Data 1989]}

The right-hand side (RHS) of the rule consists of a consequence called an "Hypothesis" in NExpert which can be True, False, or Notknown, and optional actions that occur when the hypothesis is true.

The RHS action can be used to control rule processing as an application runs. For example, consider the case in which two rules share a RHS hypothesis:
R1: LHS1 Then
Hypothesis and Action 1
R2: LHS2 Then
Hypothesis and Action2

Suppose that if R1 fires, we want any subsequent backward chaining to be exhaustive; namely, we want the system to backward chain on every rule that has the goal hypothesis, regardless of whether or not the backward chaining fails on a fired rule. If R2 fires, however, we want to backward chain only until we succeed once; at that point, the 
backward chaining halts. Action1 and Action2 would implement the different strategies with a strategy action. NExpert allows similar dynamic, or runtime, control over inheritance. Such control adds to problem solving flexibility.

Operators on the RHS consist of:

Do assigns a value to one or a list of variables. The value can be that of a single atom or of an expression;

Let assigns a string value to any variable of the same type;

Reset

strategy sets the value of its argument to the "Unknown" state;

allows the user to alter control mechanisms. It opens the strategy Dialog window for setting the inference engine and inheritance mechanism's default parameters (or initial parameters since the rules can change them). Control propagation involves constructs as contexts, backward exhaustive evaluation, RHS actions, semantic gates, inheritability, inheritance strategy, sources application, and "If Change" operator actions.

Createobject / Deleteobject

Createobject is used to create objects and/or add them to class or classes. Deleteobject deletes a link between a list of objects (class) and/or deletes an object from a Knowledge Base. These operators are part of NExpert's hybrid environment.

Execute, show same as in (LHS)

Retrieve and/or write

allows the user to read and write data from files stored on disk.

LoadRB

makes it possible to load a knowledge base incrementally, subsequent to the firing of a 
rule. Thus, all rules do not have to be loaded at the start session.

UnloadkB

unloads a knowledge base incrementally, subsequent to the firing of a rule or a metaslot action.

An NExpert rule is conjunctive in nature in that a hypothesis is true if, and only if, all the conditions are true. The truth table 5.1, shown below, provides a matrix for the state of the hypothesis given the added NExpert variable Notknown, for the union "AND" and intersection "OR" operators.

\begin{tabular}{llllllll} 
& \multicolumn{3}{c}{ AND } & \multicolumn{4}{c}{ OR } \\
& T & NR & F & & T & NR & F \\
& & & & & & & \\
T & T & NK & F & T & T & T & T \\
F & NK & NK & F & NK & T & NK & NK \\
F & F & F & F & T & T & NK & F
\end{tabular}

Key: T: True, NK: Notknown, F: False.

\section{Table 5.1 Truth Matrix for NExpert}

Notknown is rarely used. In some other expert system shells it stands for the degree of uncertainty, a logical string which implies that the user does not know the truthfulness or precision of a premise, or has no information for a given data item. It adds an extra primitive for the state of the hypothesis truthfulness.

NExpert can express disjunctive (OR) conditions with two rules pointing to the same hypothesis. When all conditions 
are true, the hypothesis is true and the actions are enabled.

As with all shells, the developer must design the NExpert rule base with inference in mind. Each rule must have at least one hypothesis that is a condition in other rules, and some rules must include conditions that are hypotheses in other rules.

\section{Classes and objects}

NExpert's object-oriented component is derived from a generic artificial intelligence tool known as a frame-based system. Frames can represent arbitrary entities, or concepts, and collections of entities. A frame can have an arbitrary number of slots (properties), each with a value. A slot may have a procedure or function as its value. Frames can occur in an inheritance network that propagates properties and values from parents to children, and vice versa, as well as multiple inheritance. An object or class can inherit properties and values from more than one source. There are two types of frames: classes, which are collections, and objects, which typically belong to one or more classes. In NExpert; slots are called "Properties". A class or an object can have many properties, and the inheritance network of objects and classes can be arbitrarily complex. Figure 5.3 partially shows the 
relationship between a NExpert class and object in which circles represent classes, and triangles represent objects.

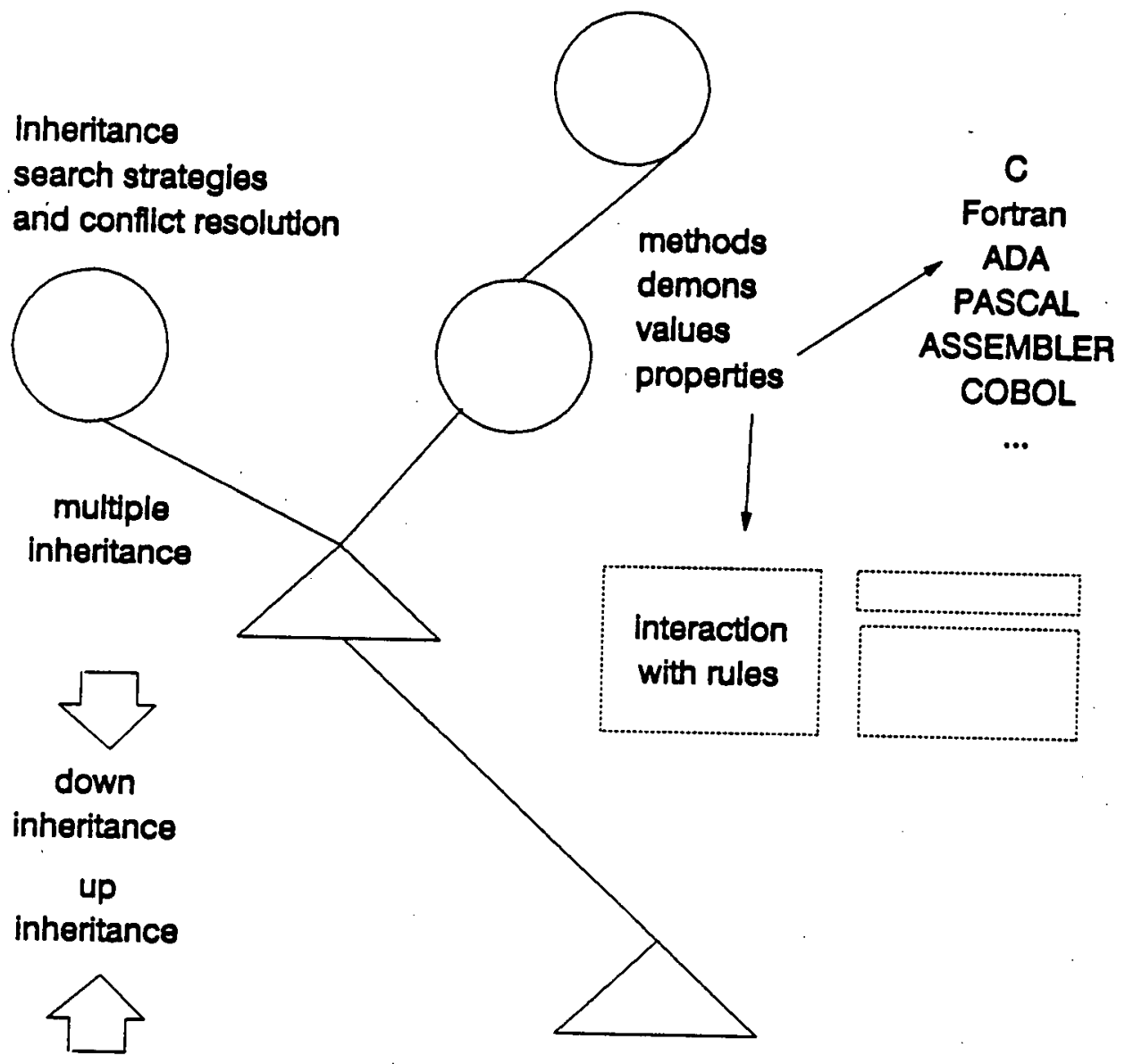

Figure 5.3 The class and object Hierarchy [from Neuron Data 1989] 


\section{Properties}

Every property of an object or class has a value, which defaults to Unknown. A property also has properties of its own called "Meta-slot" that determine its behavior, inheritance, initialization, and modifications. A meta slot can be used to implement a demon, which is a piece of code that monitors a property and reacts appropriately to changes in its value. Demons are a type of procedural attachment that can take us outside NExpert into a conventional language such as $c$. Through the "If Change" meta-slot one can have NExpert invoke a function whenever a property value is updated. A function could be used to issue a warning, return a value, or even create a new NExpert object.

\subsubsection{Viewing Rnowledge structure}

A knowledge base consists mainly of rules (LHS, RHS, and Hypothesis), frames (classes, objects, properties), text and database files, and procedures, which are accessed using "Notebooks" and "Editors". NExpert offers graphics tools, called Networks Inspector for viewing the knowledge base (rules, classes, and objects) and focusing on a piece of knowledge for analysis during development. If the viewed structure requires editing, one can zoom to it directly on the Network and use a pop-up window to change it. 
A full-screen window with a pull-down bar menu across the top allows the developer to navigate through the shell using the following: Edit, to access editing windows for knowledge elements such as rules; Expert, to load a knowledge base, start the inference process, or change the inference strategy; Inspector, to display the knowledge base graphically; and Report, to access information about the status of inference and knowledge structures. The Encyclopedia option retrieves lists of existing rules, data, hypotheses, objects, classes, and properties, in the form of alphabetically indexed pages. The windows option allows the developer to control the display of windows.

\subsubsection{The Inference Process}

Unlike most other shells, NExpert can reason using a number of strategies and has several options for beginning the inference process. Each knowledge base has a default strategy, but the developer can change this and choose between forward (event-driven) processing, backward (goaldriven) processing, or a combination of the two. Additional inference pathways are identified by keeping a list of agendas which are hypotheses of successfully fired rules.

Figure 5.4 reflects that at any given time, NExpert is focusing attention on a point that lies at the intersection of two orthogonal, but always intersecting, dimensions: 
representation and reasoning. When the system is evaluating a rule, it is necessarily concerned with structures, objects, and classes that are part of the description of the world about which it is reasoning.

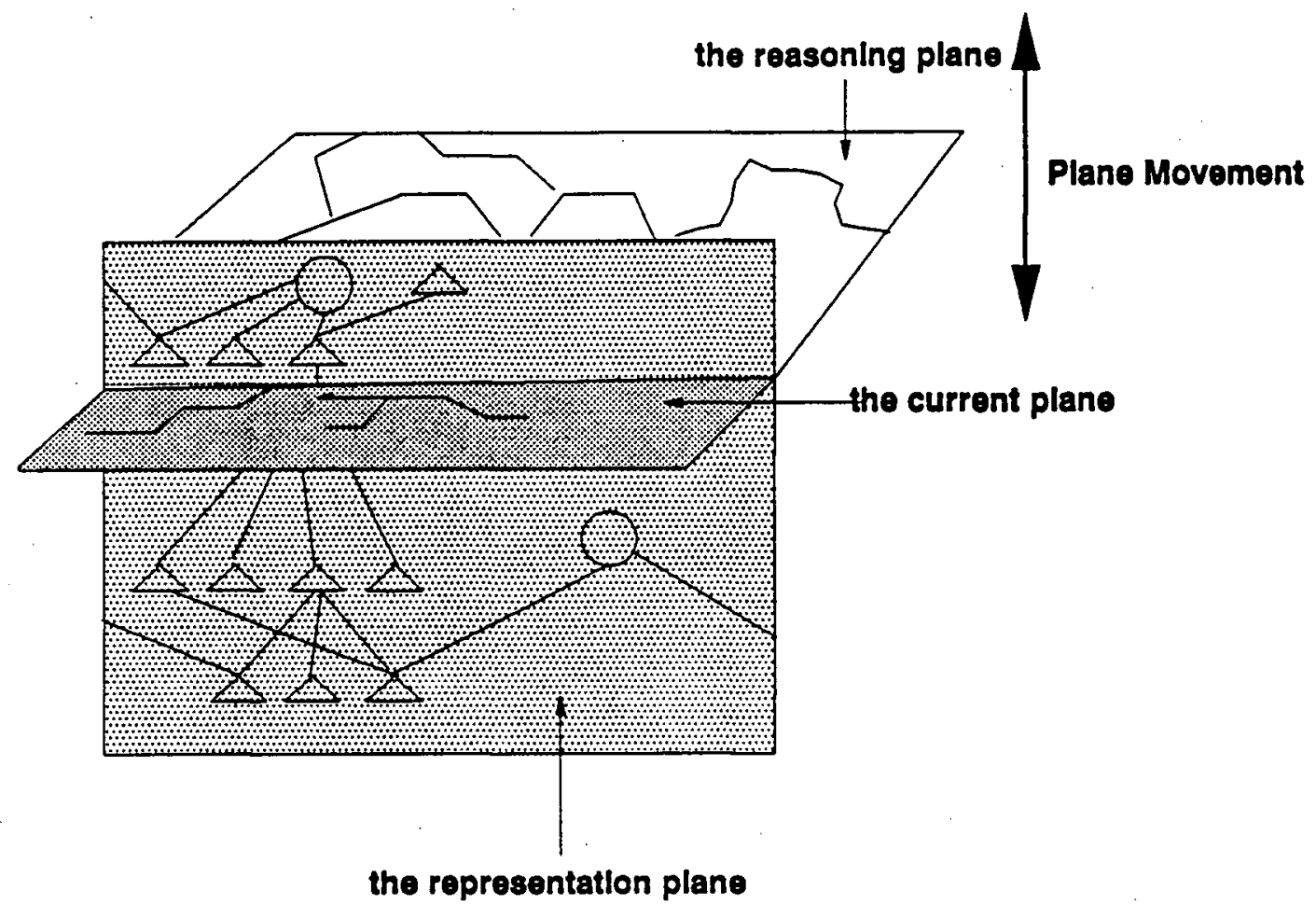

\section{Figure 5.4 Rules Perpendicular to Frames [from Neuron Data 1989]}

The user starts the inference process by selecting the "Knowcess" operator from the Expert menu, which displays the Session control window. NExpert creates a list of initial hypotheses and begins to process the rules and objects. At the same time, the system overlays several other windows on the screen to display information such as hypotheses 
currently under investigation (the hypothesis window), transcripts of any values the system has obtained or altered (the transcript window), the rules currently under investigation through backward chaining (the Rule window), and any concluded hypotheses (the Conclusions). The user can access these windows throughout the inference process.

The user can interrupt the inference engine to "Volunteer" (input) information to the NExpert system regarding whether new data are relevant to the rules and hypothesis currently under investigation. The user can start a forward-chaining process, for example, by entering all information about a problem and then letting the system fire every possible rule to develop hypotheses.

The user can suggest one or more hypotheses for the system to use to trigger the inference process. After studying the initial or suggested hypotheses, the system then identifies other hypotheses worth examining and may propagate in a backward or forward mode of chaining. For instance, figure 5.5 shows Reset operator sets a hypothesis into "Unknown" and eventually resets all preceding hypotheses to "Unknown" in a backward mode of chaining. This operator was used to reset the state of technical feasibility to "Unknown" when a combination of soil/hammer/pile fails (see figure 5.25). 


\section{Reset}

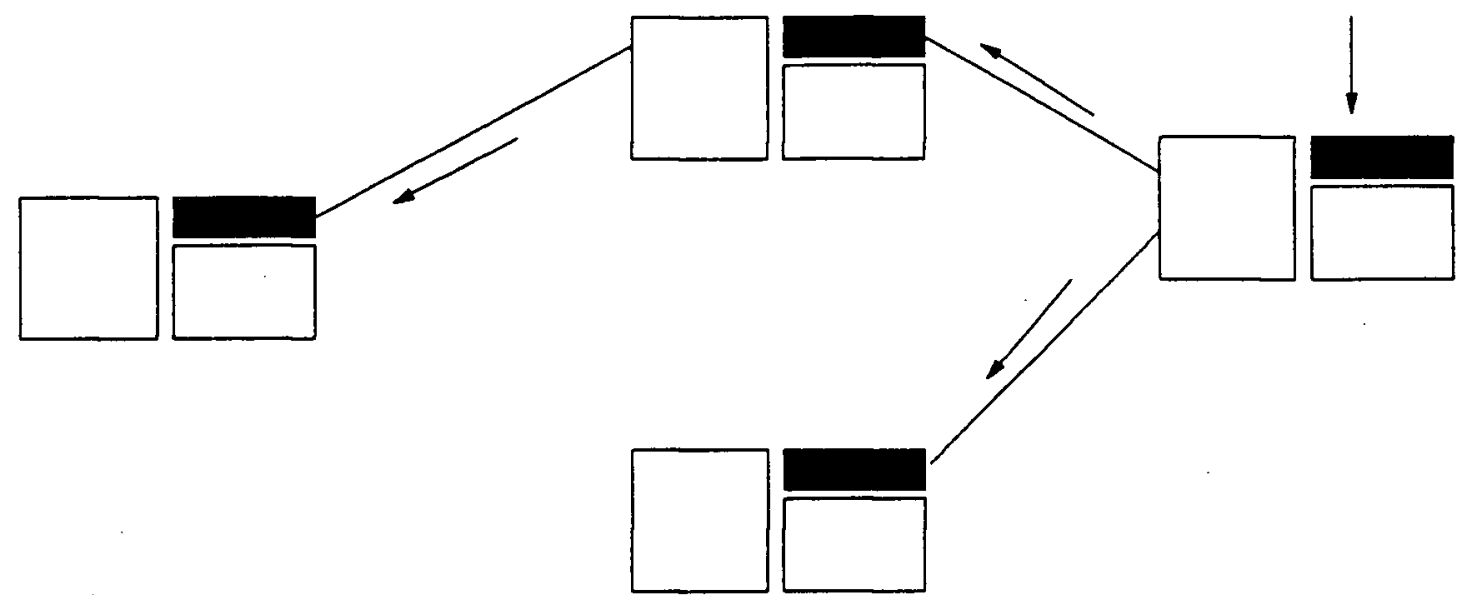

\section{Figure 5.5 Reset Operator for Inference} [from Neuron Data 1989]

A developer can use NExpert's Strategy menu, for example, to fire only the rules concerned with confirmed hypotheses, examine only data that would reject an hypothesis, or examine all data regardless of whether the associated hypothesis is under investigation. A rule can be forced to be evaluated and an object can confirm an hypothesis under investigation. With dynamic control over the inference process, one can combine all strategies to create highly complex reasoning pathways.

After the system finishes its user interrogation, the results of the inference are displayed in the conclusions window, and a trace of the system operations can be recorded on the Transcript window for debugging. Each hypothesis 
investigated is either rejected, confirmed, or already known to be true.

Rules can be grouped together and linked into related sets called knowledge islands, which can store knowledge about a large, complex domain in classes. These are templates that provide a general description of a category of items in the domain. Specific instances of each item are called objects. NExpert uses rules to reason about objects and classes. Thus, the two representation methods can be integrated and customized for the inference process for specific application needs. Figure 5.6 depicts how rules manipulate and interact with other NExpert constructs -objects, meta-slots, methods, etc. 


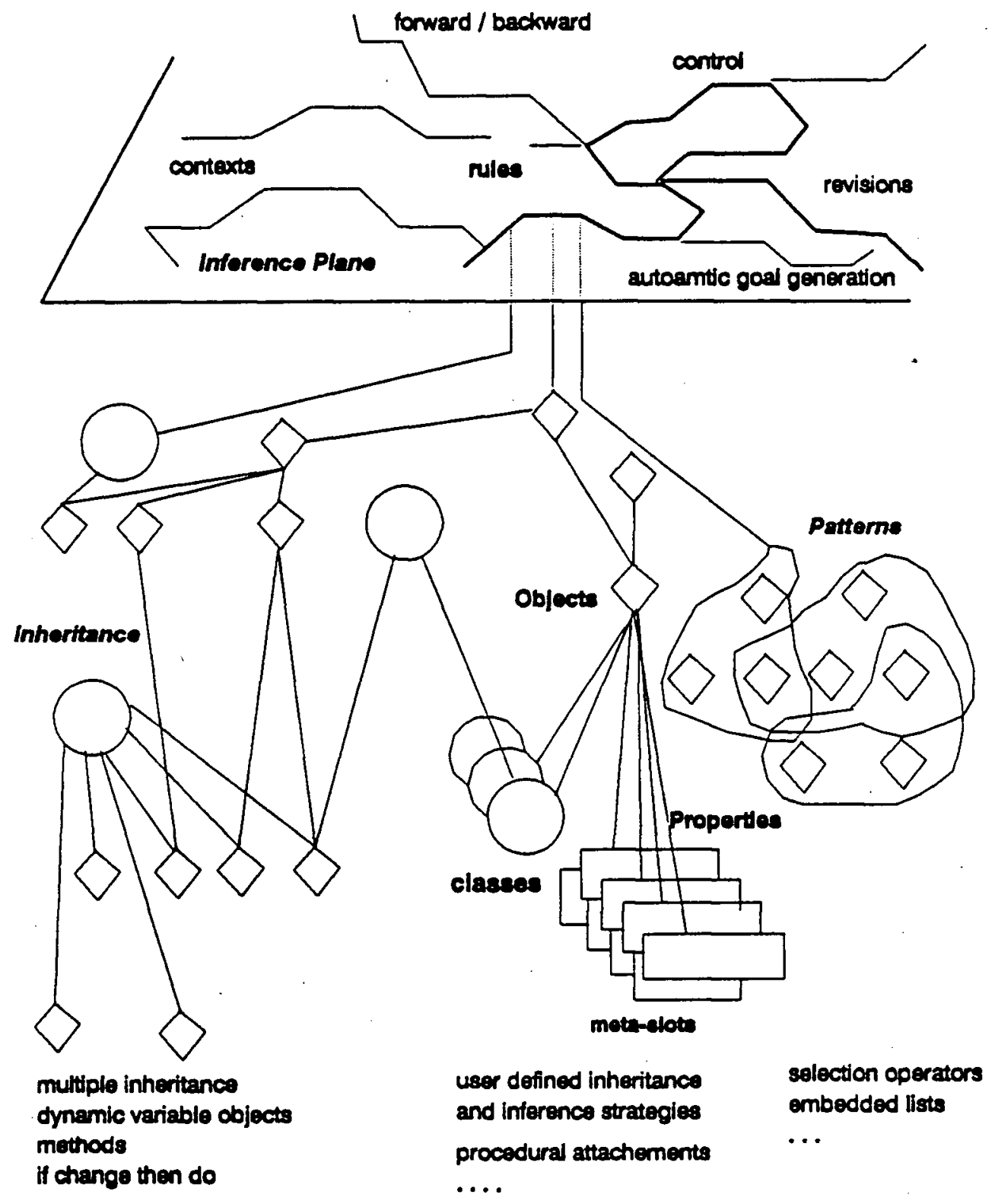

\section{Figure 5.6 Nexpert Inference Framework [from Neuron Data 1989]}




\subsection{CMSA Implementation}

\subsubsection{CMsA Overview}

The prototype system, CMSA, is an implementation of the conceptual system described in chapter 4. The CMSA control strategy and its knowledge bases are described in section 5.3.2. The latter includes procedural knowledge: pile design and pile driving production; analytical: risk analysis and evaluation criteria; heuristic knowledge: pile driver selection and sizing; and factual knowledge: sheet pile selection. Knowledge representation employs frames for method attributes (section 5.3.3); rules for the control strategy and soil context description; data bases used for structural members and construction resources; and numerical routines for design, moment, and pile driving progress prediction procedures.

The implementation of technical feasibility checks is described in section 5.3.4. Chaining and reasoning procedures used in the prototype system are described in section 5.3 .5 .

The CMSA prototype was implemented on an IBM PS2/386 personal computer equipped with 4 Mbytes of memory using the NExpert object expert system shell. The CMSA inference engine, or control strategy, acts as a methods selection shell, or skeleton, that is applicable to other methods 
domains besides the GWSS method selection for cut-and-Cover Tunnelling.

The user interface and explanation facilities for the prototype are not provided. If desired, the Screen Builder Module for NExpert could be used to enhance the interface. Text files are used extensively in CMSA to explain to the user what data is required, why it is required, and what is expected next.

The prototype implementation deals with the detailed feasibility part of section 4.3.4. The control strategy was meant to be implemented as an independent knowledge base. However, in NExpert object, this required loading and firing other knowledge bases which in turn overloaded the memory and caused a crash of NExpert object. To work around this problem, the rules from each $\mathrm{KB}$ were grouped into one knowledge base resulting in the control strategy rules being interwoven with specific knowledge rules. The risk component, due to its size, is treated separately and results are then input to the previous knowledge base. 


\subsubsection{Solution Paradigm and Rnowledge Base}

In this section, a solution paradigm of suggest, Design, Predict, and Analyze, as described by Clancey (1985), is superimposed on the augmented knowledge base to aid in the exposition.

Figure 5.7 is a restatement of figure 4.6. The solid lines correspond to the control structure implemented. The dotted lines show suggested future developments. The knowledge bases are represented by rectangles, while the enclosed dotted shapes correspond to the suggest, Design, Predict, and Analyze operators. Figure 5.8 depicts the abstract CMSA system operations cycle which is elaborated upon later. 


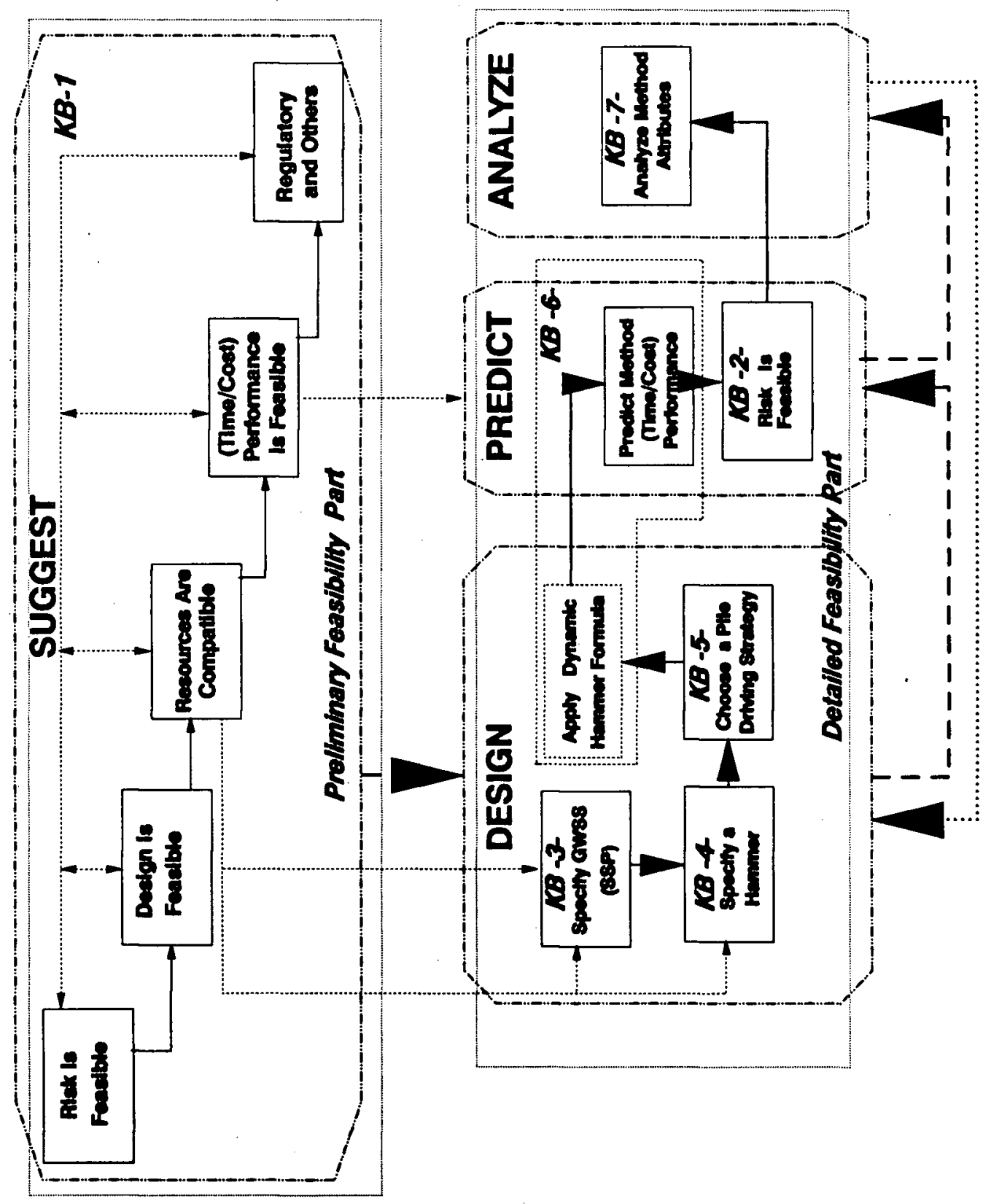

Figure 5.7 Rnowledge Base Organization and Control 8trategy 


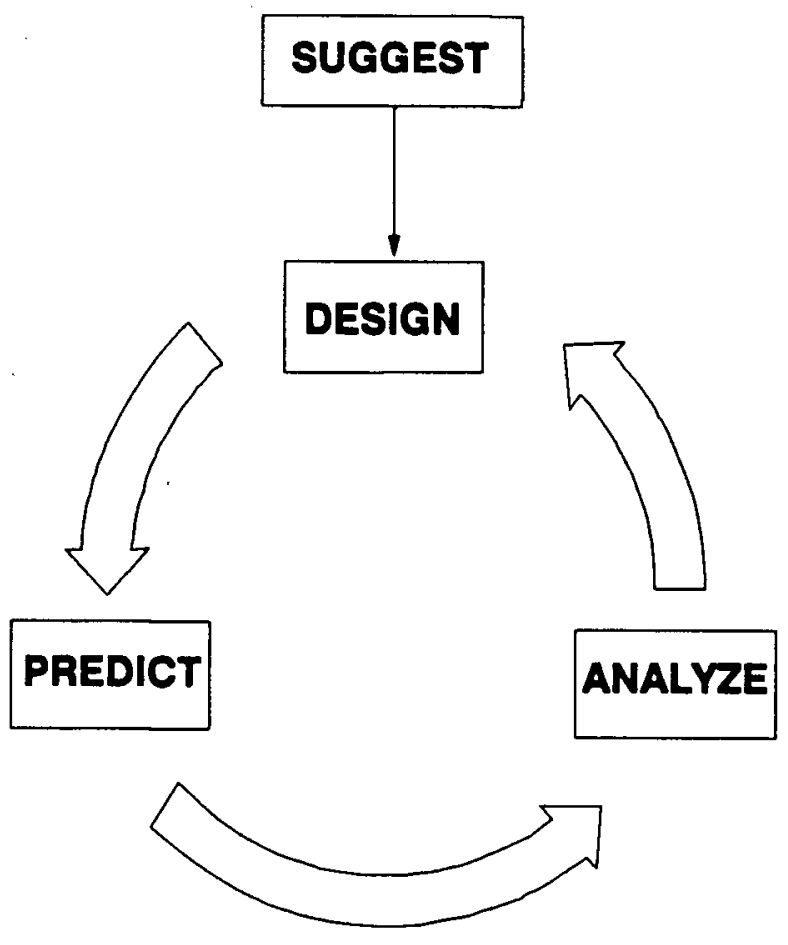

Figure 5.8 Implementation Bolution Paradigm

The foregoing operators and their corresponding knowledge bases are described below.

\section{8uggest (KB-1)}

Because no prototype was implemented for the preliminary feasibility aspect of the system, the suggest operator simply consists of a list of alternatives from which the user can select one or more candidates for the detailed feasibility analysis. The suggest operator triggers the session first by firing the design component knowledge base. 


\section{Design (KB-3, $\mathrm{KB}-4, \mathrm{~KB}-5)$}

\section{1 specify Design Elements of GWSs (KB-3)}

This component consists of two procedural knowledge components. The first one, $\mathrm{KB}-3-1$, calculates the earth lateral pressure, given a soil profile, and the resulting moments exerted on the GWSS wall. Default retaining system (back-ties or struts) spacings are required in order to perform these computations. Rules of thumb, as found in the literature (Tomlinson 1975), suggests a 15 ft vertical spacing and $12 \mathrm{ft}$ horizontal spacing between the retaining system members.

After the soil profile is described by the user, the CMSA retrieves relevant soil layer properties from a soil data base. Appendix $A$ contains the formulas utilized for calculating pressures and moments envelopes based on the approach suggested by Peck and Terzaghi (Winterkorn and Fang 1975).

The second procedural knowledge component, KB-3-2, specifies a steel sheet pile (KB-3-2-1) and soldier piles (KB-3-2-2) from their respective data bases (SSP.nxp and SPL. nxp) using the section modulus for the sheet piles and soldier piles derived from $\mathrm{KB}-3-1$ moments. Currently, a strength criterion is checked. Deflection and buckling considerations are not considered. 
In $\mathrm{KB}-3-2$, structural member selection is based on aggregating sections prudently into groups defined by their upper and lower bounds. This is a commonly used design approach in developing expert systems for engineering design (Adeli and Paek 1986) .

The design of retaining system members (struts, wales, lagging) for the GWSS is also treated in $\mathrm{KB}-3-2$. Strut and wale design knowledge is based on moment criteria. Lagging design is based on Canadian standards Association criteria (1976) .

Conversely, retaining system structural members are considered to be fixed variables for all Gwss. In the future, $\mathrm{KB}-3-3$, could be utilized to investigate the tradeoff between the retaining system cost and the cost of the GWSS piles.

Once $\mathrm{KB}-3$ is successful in specifying sheet and soldier pile sections, control moves to selection of a hammer (construction resource).

\subsection{Select construction Resources (KB-4)}

Resources employed for sheet piling include: a pile driver, a crane, a pile frame, and pile driving crew. Crane size, in tons, is proportional to the weight of the hammer and its attachments. 
For labelling purposes, note that $\mathrm{KB}-4-1$ and $\mathrm{KB}-4-2$ of figure 4.6 are combined into one component of $\mathrm{KB}-4$ for specifying impact hammers. For knowledge base organization purposes (see table 5.2), $\mathrm{KB}-4-1, \mathrm{~KB}-4-2$, and $\mathrm{KB}-4-3$ refer to impact hammers, vibratory pile drivers, and cranes knowledge base components respectively.

\subsubsection{Impact Hammers Specification (KB-4-1)}

This component of the knowledge base contains factual and experiential knowledge in order to specify a hammer. Several types (Single and Double Acting Air Hammers) and sizes of hammers are stored in separate data bases (e.g. DAAH.nxp for Double Acting Air Hammer).

The following hammer specification heuristics are employed.

1. Determine the class or type of hammer based on the soil profile (see section 4.3.2).

2. Use the experiential rule of proportioning the hammer size equal to or less than the steel pile cross section area multiplied by a factor of 3000 . This is to provide an upper bound on the hammer size in order not to damage the pile, and corresponds to a commonly used rule of thumb in the industry.

If the class of hammers determined in step 1 does not satisfy the condition in step 2 , then another class should be selected and tested. 


\subsubsection{Vibratory Hammers specification KB (KB-4-2)}

The vibratory hammer differs substantially from the impact hammer in its operating principles, therefore, the selection criterion is different. A vibratory pile driver can be specified in terms of its dynamic force and amplitude. Under favorable conditions, a vibratory hammer is 4 to 8 times faster than an impact hammer; however, it is more expensive to rent and operate.

In the prototype system, emphasis has been placed on sizing the impact hammer, since a predictive model exists in the form of a dynamic formula for a subclass of its hammers. This model allows prediction of production, for a given soil/hammer/pile scenario, and indicates whether or not a pile can be driven to its refusal. A similar predictive model for the vibratory hammer does not exist.

Crane attributes are dependant upon selected hammer size, pile length, etc (see Appendix C). Currently, it is treated as a fixed variable, although experiential knowledge pertaining to crane selection is included in $\mathrm{KB}-4-3$.

\subsection{Construction strategy selection (KB-5)}

For driving of sheet piles, construction strategy refers to the pattern of pile driving. For the prototype, this is simplified into driving sheets either as singles or in doubles. 
The selection of a construction strategy involves tradeoffs among several factors: soil stratification and conditions; pile driver size; pile capacity and size; pile segment length; pile driving fixed time (positioning, splicing); and variable driving time. Soil stratification and conditions are the dominant factors.

If the soil stratification and conditions state can be characterized as soft, CMSA attempts to drive piles in doubles. If the doubles configuration can not be driven to refusal, then CMSA retracts this construction strategy and explores driving in singles.

To predict the feasibility of whether each strategy, and other method attributes, are most suitably synthesized, a test is required for the foregoing stated conditions. This is elaborated on in the technical feasibility section.

3. Predict $(\mathrm{KB}-6)$

This operator basically decides the appropriate construction process model to be used. For CMSA, the dynamic formula derived in Appendix $B$ is applied only after a sheet pile section and hammer have already been selected. For a given scenario of soil/hammer/pile, the rate of pile penetration decreases with pile depth. A routine, named "Drive.c", described in section 4.3.4, is used to predict the incremental velocity of pile driving and to check if the refusal depth is achieved. If the latter is achieved, then 
this routine returns a "True" value for the technical feasibility state and the total penetration time for a single pile. From the latter, CMSA computes total pile driving production, including fixed time, and thus production cost. Total cost computation including quantity take-off for the design elements, pile driver and crane, and labor costs is then done.

Risk assessment (KB-2) involves application of a rule based analytical risk assessment based on the approach described in section 4.4. This component has been programmed as a separate routine since it requires substantial user input (over 30 data input parameters for the user to volunteer).

This component returns the expected risk cost for each alterative which is then incorporated in the total cost computation for the alternative.

\section{Analyze $(\mathrm{KB}-7)$}

The current method being evaluated by CMSA is then analyzed to see if it satisfies the target project time/costs. If it does, then it is accepted. Otherwise, recommendations to alter method attributes to achieve feasibility are suggested by the system. CMSA may carry out some of those recommendations, by backtracking and changing the state value of a method attribute to permit re-evaluation (re- 
iterate) by either the suggest or Design processors, followed by the Predict processor.

\section{Control strategy (KB-8)}

The control strategy is the skeleton of the system, and is responsible for managing the problem solution strategy. In the CMSA, the control strategy is intertwined with other knowledge bases in one overall knowledge base. In designing the CMSA implementation design strategy separate KBs were developed so that they could be run independently of each other. The reasons for this strategy include:

1. The user may choose to conduct a risk appraisal for an alternative without going through the interrogation. For instance, if one wants to assess the risk status of a secant pile alternative, the risk feasibility component $(\mathrm{KB}-2)$ can be independently fired.

2. Some KBs are incomplete in their development. For instance, the vibratory hammer $\mathrm{KB}(\mathrm{KB}-4-2)$ incorporates experiential knowledge for hammer selection, but no process for predicting a production rate because no algorithm exists.

3. Integration of independent knowledge bases, including the control strategy meta-rules, into one knowledge base proved to be cumbersome. With NExpert object, such integration requires the control strategy to execute operators "Load KB" and "Unload KB" to fire other KB's. Invariably, this creates a system crash because memory is overloaded.

So the strategy adopted was: develop independent KBs for testing separately; then merge into one overall KB for purposes of developing a working prototype CMSA. 
4. From experience in developing the prototype system, it is easier to develop and edit each knowledge base independently, which permits an incremental approach to development of a system.

Table 5.2 shows summary statistics for the CMSA knowledge bases shown in figure 5.7. The last column indicates the number of rules extracted from the individual knowledge bases for purpose of assembling the prototype.

Knowledge Base Number and Name

(KB-1) Preliminary Feasibility

(KB-2) Risk Component Feasibility

Wall Design Elements

(KB-3-1) Earth Pressures and Moments

Structural Members

(KB-3-2-1) Steel Sheet Piles

(KB-3-2-2) Soldier Piles

Hammer/Crane Selection

(KB-4-1) Impact Hammer Selection

(KB-4-2) Vibratory Hammer Select

(KB-4-3) Crane Selection

(KB-5) Construction Strategy Selection

(KB-6, KB-7) Predict and Analyze

(KB-8) Control and others

Total Rules

\section{KB Size ${ }^{10}$}

Number of Rules for each KB

$\begin{array}{ll}10 & 2 \\ 18 & 4 \\ 5 & 3 \\ & \\ 18 & 18 \\ 18 & \\ & \\ 16 & 16 \\ 15 & \\ 6 & \\ 15 & 15 \\ 10 & 10 \\ & 10\end{array}$

Number of Rules in CMSA

2

3

18

16

15

10

78

\section{Table 5.2 Knowledge Base statistics}

${ }^{10}$ Number of rules is an indirect measure of knowledge base size. The number of rules is a function of system objectives, control strategy required, ES shell representation schemes, knowledge organization, and the way rules are collapsed on each other. The rule construct in NExpert object is a high level operator that in comparison requires several rules to emulate in other purely rule based system. 


\subsubsection{Rnowledge Representation}

The prime representation constructs in CMSA are frames for method attributes and rules for soil profile and conditions, performance criteria evaluation, (quantity take-off and cost estimate) and the control strategy. Analytical routines are used for pile driving progress rate prediction, and pressures and moment computation. Data bases are used to store the properties of structural members (piles, wales, struts), hammers, and soil types. When the CMSA retrieves data from a data base, it assigns a corresponding class and object hierarchy. An object corresponds to a record, and object attributes correspond to a record field in NExpert.

\section{Soil Profile}

As shown in figure 4.7, a maximum of two soil layers and four soil types: sand types (Loose_Sand, Dense_Sand) and/or clay (soft clay, stiff clay) were treated. Soil types are represented as database records. Each record corresponds to a soil type object, and the record fields correspond to object attributes.

In section 4.3 .2 , two forms of entering soil information were identified. The first is used when the user is asked to identify one soil type per layer: e.g. dense sand for the top layer and stiff clay for the bottom layer. Given this input, the system then retrieves the Dense_Sand record 
from the soil database to obtain the saturated unit weight, angle of internal friction, and other properties.

For the second mode of input, if the user has a standard penetration test log (SPT) for the soil profile, then CMSA identifies the soil layers against SPT values (e.g. if SPT is high and soil is sand, then this is a Dense_Sand).

The first soil input format is used for calculating pressures and moments in order to size the piling. The second soil input format is used for predicting the pile driving rate using the Drive.c routine.

\section{Method Attributes}

Frames, called objects in NExpert object, were used to represent method attributes, with emphasis being placed on design elements and construction resources. Construction strategy and construction model processes have been treated as predicates of object-attributes-values in a rule format. In future work, it is suggested that they be treated using a frame representation.

\subsection{Design Element}

A design element in CMSA corresponds to a member of the ground wall support system: i.e. steel sheet pile, soldier pile, wale, strut, and lagging. Figure 5.9 displays an overall hierarchy of design element classes and objects. 
A generic class of design element, a parent, has universal slots or attributes that can be inherited by its children (descendants). Attribute values are either inherited, defaulted, or evaluated via a procedure.

Descendants of this global class are a design element subclass of either the GWSS subclass and/or the retaining system subclass. Further branching from the GWS includes subclasses for the GWSS of the type of structural member types.

To illustrate the foregoing, consider the steel piling alternative. Sheet piles can be either steel, wood or concrete, each of which have a set of material properties, such as modulus of elasticity, yield strength, bending stress, unit costs, and so forth. For example, steel sheet pile properties include moment of inertia, allowable bending stress, cross section area, type of alloy material and designation. In the CMSA, some of these properties are required in the approximate design routines to establish construction resource compatibility, and for quantity takeoff purposes. 

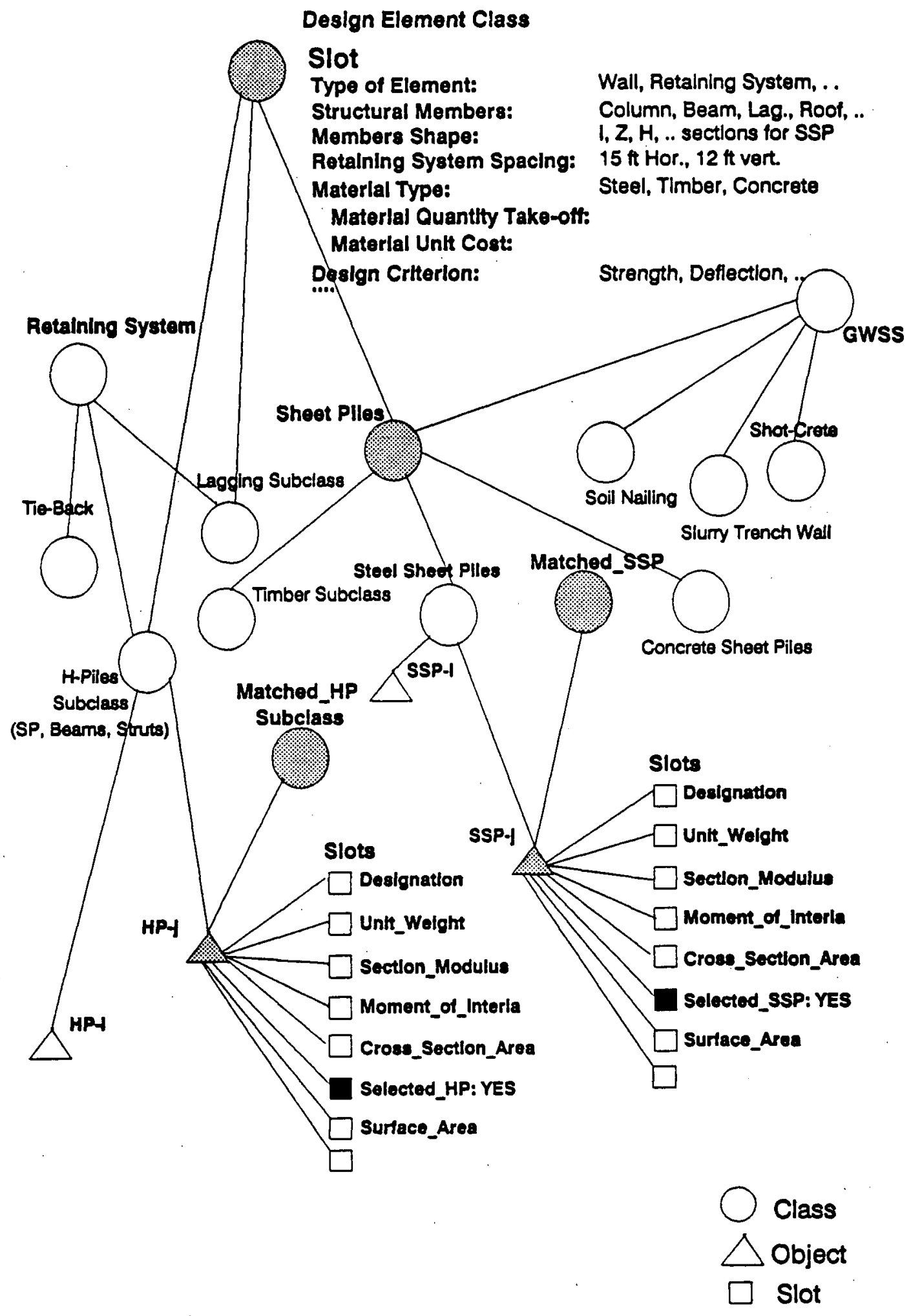

Figure 5.9 Design Element Class Hierarchy 
A subclass of steel sheet pile consists of several steel pile objects (SSP_1, SSP_2, SSP_n) whose attribute values include designation, unit weight, section modulus, cross section area, and surface area. Unit weight is used to estimate the total cost (unit cost in $\$ /$ ton); section modulus is used to satisfy a strength criterion; cross section area is used in heuristic rules to assess the damage potential from driving; and surface area is used in the skin friction resistance calculation to estimate pile driving speed. Figure 5.10 shows a steel sheet pile frame format.

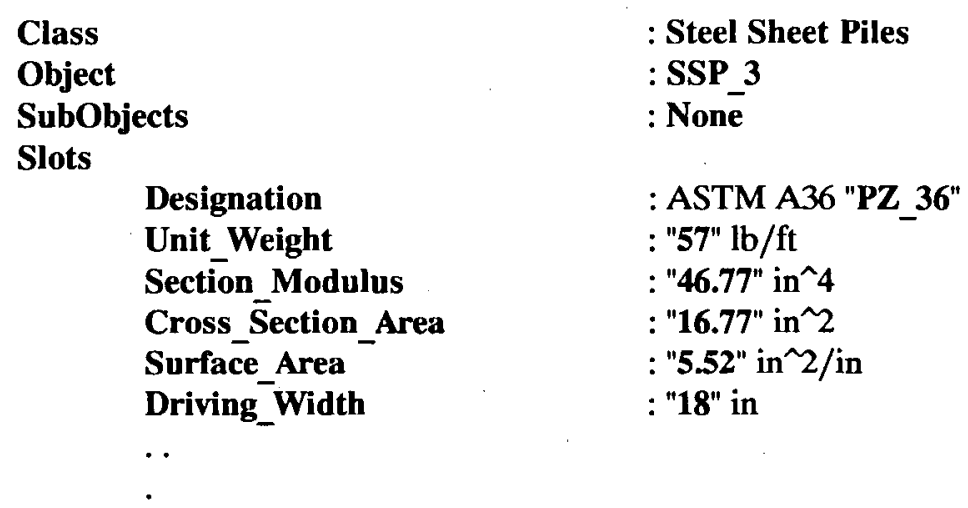

\section{Figure 5.10 Design Element Instance Frame}

Values in key fields for steel sheet pile records, stored in the sheet piling data base, are retrieved by the CMSA and inserted into the appropriate slots of the design element frame. This representation scheme is equally applicable to other design elements such as $H$ piles, sheets, wales and lagging. A partial design element data base is presented in Appendix D. 
An example of the steel sheet pile class representation implementation using NExpert is shown in figure 5.11 for the selected SSP.

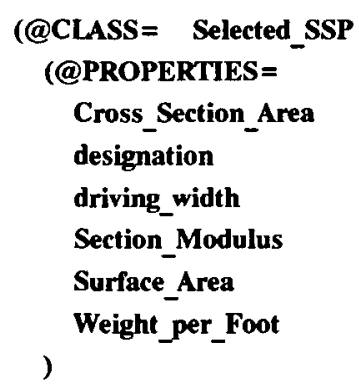

\section{Figure 5.11 steel sheet Pile class in NExpert}

All sheet piles in the database are first retrieved and loaded into the selected_ssp class using the rule shown in figure 5.12. This is a factual rule, based on judicious section modulus range partitions, which results in an SSP from the list of Selected_SSP being chosen as a Matched_SSP. CMSA, thereafter, fires such a rule to pick the sheet pile that satisfies the design strength criteria based on the section modulus, which is then saved in the Matched_SSP Class for later treatment.

Steel sheet pile sections are stored in groups, with each grouping having a minimum and a maximum section modulus. A preliminary sizing is used to fire a rule so that NExpert retrieves members of the group. Rules are then fired in order to select a specific member of the group that satisfies the strength criterion. 
The NExpert text rule syntax for selecting a steel sheet pile is shown in figure 5.12 which is fired after steel sheet piles are retrieved and stored in the selected_SSP.

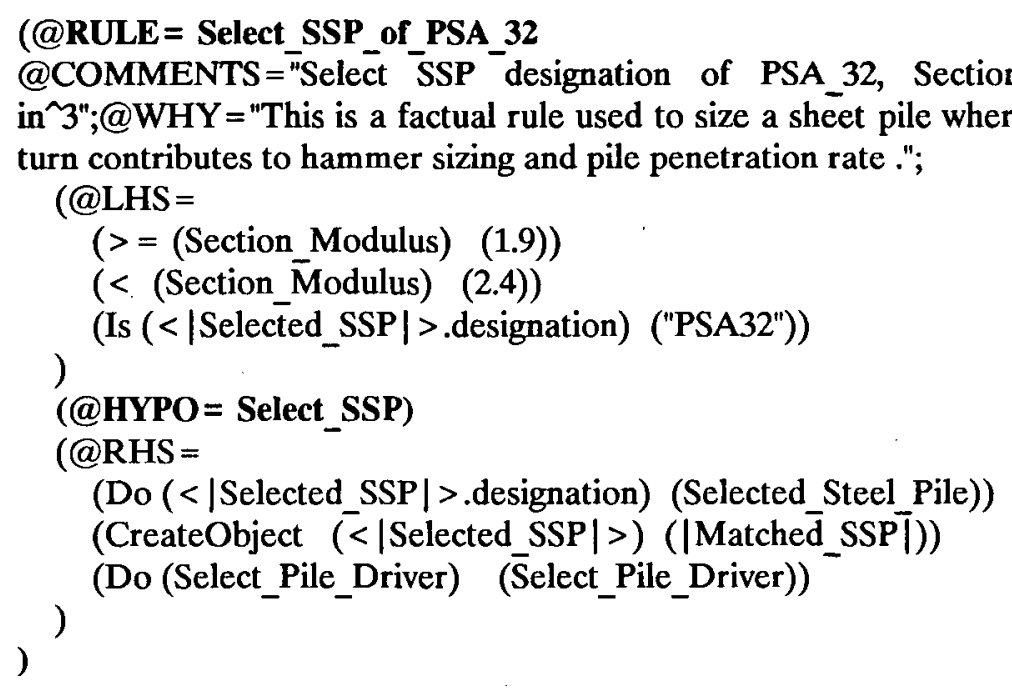

Figure 5.12 steel sheet Pile selection Rule

This rule is interpreted clause by clause as follows.

\section{Left hand side}

The first and second clause say that if the required section modulus (which is calculated by a prior rule) for a sheet pile is between $1.9 \mathrm{in}^{3}$ and $2.4 \mathrm{in}^{3}$, and if the third clause says there is a sheet pile object in the selected_SSP that satisfies those section modulus limits, then "PSA_32" of A36 ASTM is selected.

The \langle| selected_ssp $\mid>$ notation implies a pattern matching operator for sheet piles which are treated as objects in a list in order to match whatever property is specified in the LHS . 


\section{Hypothesis}

The hypothesis ("Hypo") name called select_sSP is invoked by "backward chaining" via the control strategy. If the Left Hand Side conditions were satisfied, then this hypothesis is evaluated as TRUE: otherwise it is FALSE or NOTKNOWN if one of the conditions was false or not known respectively.

\section{Right Hand side}

The "Do" operator assigns the designation of a steel sheet pile to the variable "Selected_steel_Pile", and then uses the "Createobject" operator to link the selected sheet pile (Selected_SSP) to a new class or list of "Matched_SSP", to separate it from the rest of the sheet piles for subsequent use in hammer selection and technical feasibility. The Matched_SSP object inherits its attributes and values from the Selected_SSP class (pattern matched sheet pile). The last clause of the "Do" operator transfers control in order to invoke the "Select_Pile_Driver" hypothesis using backward chaining. This hypothesis is then used to select a hammer that satisfies soil and pile conditions including the Matched_ssP properties which are treated as constraints. NExpert text database Format (*.nxp), flat database, for the steel sheet piles is shown in figure 5.13 . 
\SSP 1.Designation $\backslash="$ PZ38"

\SSP-1. Weight_per_foot $\backslash=" 57.00 "$

ISSP 1.Cross section area $\backslash=" 16.77^{n}$

\SSP_1.Driving_width $\widehat{\top}=" 18^{\mathrm{n}}$

\SSP_1.Surface_area $\mid=" 5.52^{n}$

\SSP_1.Section_modulus $\backslash=" 46.8$ "

\SSP 2.Designation $\backslash=$ "PZ32"

\SSP-2.Weight_per_foot $\backslash=$ "56.00"

\SSP 2.Cross_section_area $\backslash=" 16.47^{n}$

\SSP 2.Driving_width $\rceil=" 21^{n}$

\SSP_2.Surface area $\backslash=" 5.52^{n}$

$\backslash$ SSP_2.Section_modulus $\backslash=" 38.3^{n}$

$\cdot$

\section{Figure 5.13 steel sheet Piles Database (SSP.NXP)}

\subsection{Construction Resource Class Hierarchy}

Figure 5.14 shows a construction resource hierarchy which divides cut-and-Cover tunnelling capital intensive resources into classes and objects. A resource class has universal slots for activity and task type and identification, unit cost, productivity, etc. The subclasses of bulldozers, cranes, and hammers have further slots to characterize them in terms of their functionality, size, operational properties, etc. For instance, for a GWSS of steel sheet piles, bulldozers are used for "clearing" and excavation activities; and cranes are used for handling and hoisting materials, muck removal, and carrying a hammer for the pile driving activity. 


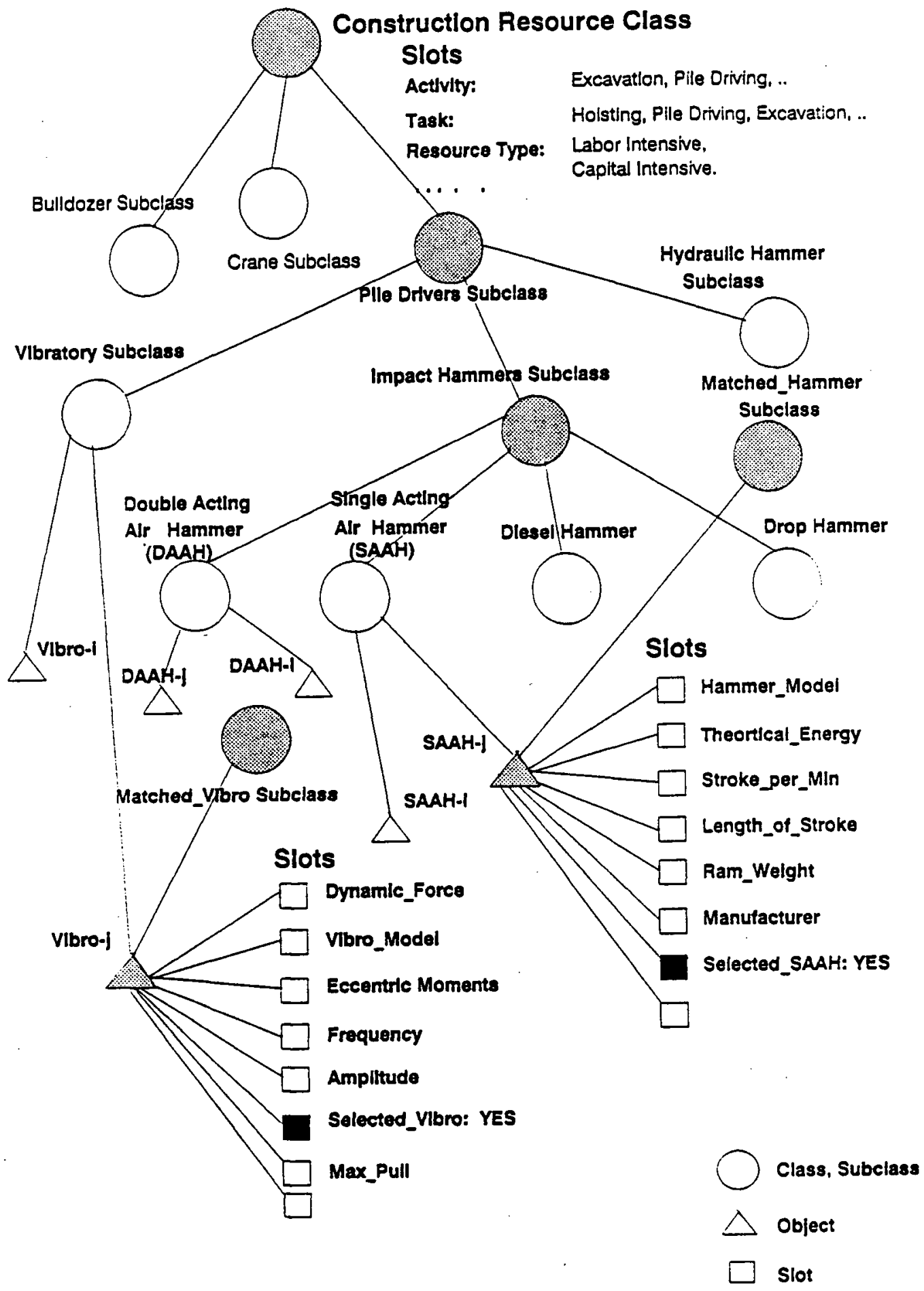

Figure 5.14 Construction Resource Class Hierarchy 
The hammer subclass was divided into impact hammers, vibratory hammers and hydraulic hammers. Impact hammers were further classified in accordance with their operating mode as single acting air hammer, double acting air hammer, diesel hammer, differential acting hammer and drop hammer.

Each hammer type is represented by a frame, an example of which is shown in figure 5.15 .

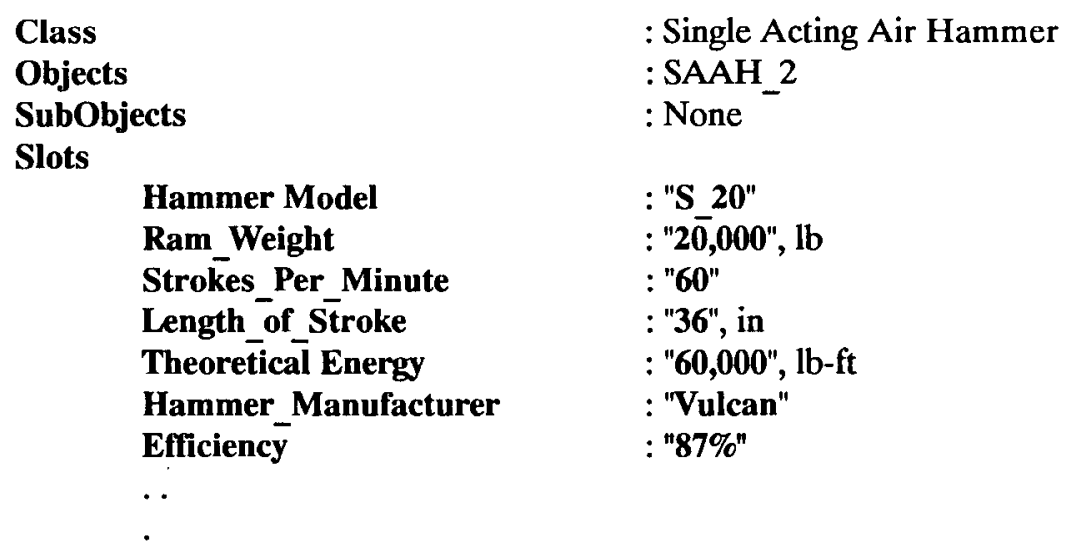

\section{Figure 5.15 Impact Hammer Element}

Suppose that the soil context variables and resource availability suggest use of a single acting air hammer (SAAH). Then, a database for the SAAH subclass would be retrieved and required record attributes would be mapped into the corresponding object and properties in NExpert. The construction knowledge for hammer selection and performance was encoded in a rule, which involves retrieving soil stratification information and pile properties from the 
pile frame via a message which sends the selected pile cross section to a heuristic rule which limits the maximum size of the hammer.

In addition to developing a data base for single acting air hammers, data bases were created for double acting air hammers as well as vibratory ones. This permits one to explore the efficiency of the prototype system to select the most appropriate resource as a function of soil context. An example frame for a vibratory hammer is shown in figure 5.16 .

Knowledge for selecting and sizing a vibratory hammer is described in Appendix D. The prediction of production rates requires heuristic knowledge, and is done by mapping vibratory hammer type and size versus soil stratification.

Class

Objects

SubObjects

Slots
: Vibratory

: Vibro_1

: None

: "1412"

: "20,000", lb

: "650", HP

: "400" , Vibration per min

: "1.5", in

: "80", Tons

: "10.20", lb

: "20.5", lb

: "ICE"

: "93\%"

\section{Figure 5.16 Vibratory Pile Driver Element}


The data bases developed for the double acting air hammer types and vibratory hammer types are shown in figures 5.17 and 5.18 respectively.

$\backslash$ Hammer_01.Hammer_Model $\backslash=" 2 "$

\Hammer_01.Ram_Weight $\backslash=" 3000 "$

$\checkmark$ Hammer_01.Strokes_per_Min $\backslash=" 70 "$

$\backslash$ Hammer_01.Length_of_Stroke $\backslash=" 29^{\prime \prime}$

\Hammer_01.Thero_Energy $\backslash=" 7260 "$

$\backslash$ Hammer_02.Hammer_Model $\backslash=" 1 "$

$\backslash$ Hammer_02.Ram_Weight $\backslash=" 5000 "$

$\backslash$ Hammer_02.Strokes_per_Min $\backslash=" 60 "$

$\backslash$ Hammer_02.Length_of_Stroke $\backslash=" 36 "$

$\backslash$ Hammer_02.Theor_Energy $\backslash=" 15000 "$

....

$\cdot$.

Figure 5.17 Double Acting Hammer Database (DAAH.NXP)

|Vibratory_1.Dynamic_Force $\backslash=$ "204"

\Vibratory_1.Model \= "1412"

|Vibratory_1.Manufacturer $\mid=$ "ICE"

\Vibratory_1.Frequency $\backslash=" 1200 "$

|Vibratory_1.Amplitude $\backslash=" 1 "$

\Vibratory_1.Horse_Power $\backslash=$ "650"

\Vibratory_1.Max_Pull_Extract $\backslash=" 80 "$

|Vibratory_1.Pile_Clamp_Force $\backslash=" 250 "$

|Vibratory_1.Suspended_Weight $\backslash=" 10.20 "$

$\mid$ Vibratory_1.Shipping_W $\overline{\text { Weight }} \backslash=" 20.5 "$

|Vibratory_1.Dynamic_Force $\backslash=$ "204"

\Vibratory_1.Model $\backslash=$ "1412"

|Vibratory_1.Manufacturer $\backslash=$ "ICE"

|Vibratory_1.Frequency $\backslash=$ "400"

$\mid$ Vibratory_1.Amplitude $\backslash=" 1.50 "$

|Vibratory_1.Horse_Power $\backslash=$ "650"

\Vibratory_1.Max_Pull_Extract $\backslash=" 80 "$

|Vibratory_1.Pile_Clamp_Force $\backslash=" 250 "$

|Vibratory_1.Suspended_Weight $\backslash=" 10.20 "$

\Vibratory_1.Shipping_W Weight $\backslash=" 20.5 "$

..

Figure 5.18 Vibratory Hammer Database (VIBRO.NXP) 
The NExpert text format for the selected impact hammer class is shown in figure 5.19 .

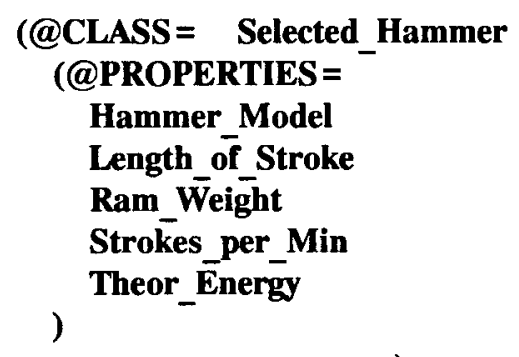

Figure 5.19 Impact Hammer Class in NExpert

Shown in figure 5.20 is an impact hammer selection rule. This rule is fired if previous rules have indicated that a single acting air hammer may be suitable. The hammer selected (if one is feasible) is linked to Matched_Hammer class for later use. The Matched_Hammer inherits the same attributes and values of the "pattern" <|Selected_Hammer $\mid>$. 
(@RULE $=$ Select_Vibratory_PD_or_DA_Hammer_PD_for_LS_ON_STCL @COMMENTS="Select a hammer type based on soil profile and conditions (Using Hunt 1979 Table)";@WHY="Inference category for this rule is set to 1 since double acting air hammer (DAAH-Hammer) overrides the vibratory selection under this rule condition (assumption)";

(@LHS =

(Is (Soil_Profile_Scenario) ("Loose_Sand_ON_Stiff_Clay"))

(Retrieve ("daah.nxp")

(@TYPE=NXP;@FILL=ADD;@CREATE=|Selected_Hammer $|;|$

)$)$

$(<=\quad(<\mid$ Selected Hammer $\mid>$. Theor Energy

( $\mid$ Matched_SSP $\mid$.Cross_Section_Area * 3000)

(Name- $($ MAX $(<\overline{\mid}$ Selected_Hammer $\mid>$.Theor_Energy)) (Max_Energy))

)

$\left(=(<\mid \text { Selected_Hammer } \mid>\text {.Theor_Energy-Máx_Energy) }(0))^{-}\right.$

(@HYPO= Select Pile Driver)

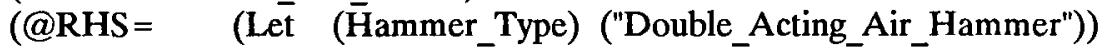

(CreateObject $(<\mid$ Selected_Hammer $\mid>)$ ( $\mid \overline{\text { Matched_Hammer } \mid))}$

(DeleteObject $(<\mid$ Selected_Hammer $\mid>)$ (|Selected_Hammer $\mid))$

(Do (<|Matched_Hammer $\mid>$.Hammer_Model) (Hammer_Model))

)

(Do (SP_Production) (SP_Production))

\section{Figure 5.20 Hammer selection Rule in NExpert}

The rule in figure 5.20 can be interpreted clause by clause as follows:

\section{Left Hand side}

1.1 The first condition checks the soil profile scenario that dictates the type and/or class of hammers that should be used (Impact versus .Vibratory, Double_Acting_Air_Hammer (DAAH), Diesel_Hammer, etc. Suppose, based on experience, that a soil profile of loose sand on stiff clay is best handled using an impact hammer, and within this subclass, a double acting air hammer is the preferred choice. 
1.2 If the first condition is successfully satisfied (returns True), the second condition assumes that the DAAH subclass best matches the soil profile. Therefore the database of $\mathrm{DAAH}$ will be retrieved and attached to Selected_Hammer Class.

1.3 The third condition reduces the list of Selected_Hammers by retaining only those hammers which have a theoretical energy $(l b-f t)$ equal to or less than 3,000 times the cross sectional area of the Matched_SSP. This rule of thumb is used in the field by pile driving contractors to prevent steel sheet pile damage caused by over-sized pile driving equipment. The driving strategy state is assumed here to be "In_Singles". However, if it is "In_Pairs", then the upper bound is doubled. There is a tradeoff between the driving strategy and hammer size. Furthermore, the hammer weight may dictate the weight of the pile segment (length and weight), especially for the case of a vibratory pile driver.

1.4 One hammer selection criterion is to pick the most productive, or largest, hammer in terms of its theoretical energy (cost is not considered at thislevel) assuming those hammers have the same hammer efficiency. Different models within same hammer subclass have different hammer efficiencies for 
pile/soil/hammer scenarios. Therefore, condition four picks the largest hammer from the selected_Hammer list using NExpert operator "MAX" .

2. The hypothesis named select_Pile_Driver is triggered through "Forward chaining" via the control strategy. If the Left Hand side conditions were satisfied, then this hypothesis is evaluated as "True"; otherwise it is "False" or "Notknown" when one of the conditions was Notknown.

\section{Right Hand side}

The "Let" operator assigns the hammer subclass type as a string, Double_Acting_Air_Hammer, to the Hammer_Type variable.

The "Createobject" operator links the hammer which satisfied the previous conditions to the Matched_Hammer class. The "Deleteobject" operator deletes the Matched_Hammer from selected_Hammer if this Matched_Hammer failed to produce the required production rate, as inferred from the technical feasibility diagnosis.

The "Do" operator then assigns the hammer model designation to a variable of Hammer_Model for subsequent treatment. The last "Do" operator transfers control to the SP_Production hypothesis where the 
pile/soil/hammer combination is examined for technical feasibility.

\section{Construction strategy class Hierarchy}

For the Cut-and-Cover tunnelling problem, the construction strategy class can be described in terms of a hierarchy (see figure 5.21). At the higher levels, overall construction approaches, such as top-down or bottom-up (e.g. the Milano method), are treated. These high level strategies greatly influence lower level strategies, activities and their sequencing .

For instance, for a top-down strategy using a steel sheet pile GWSS, strategies at the pile driving activity level are drive "In_Singles" or "In_Pairs". CMSA deals only with the lower level strategy, whereas the higher level strategies are assumed to be fixed.

\section{Construction Process Model class}

The construction process model (CPM) draws on selected design and resource frames in a process aimed at satisfying certain constraints and quantifying construction method performance measures. 


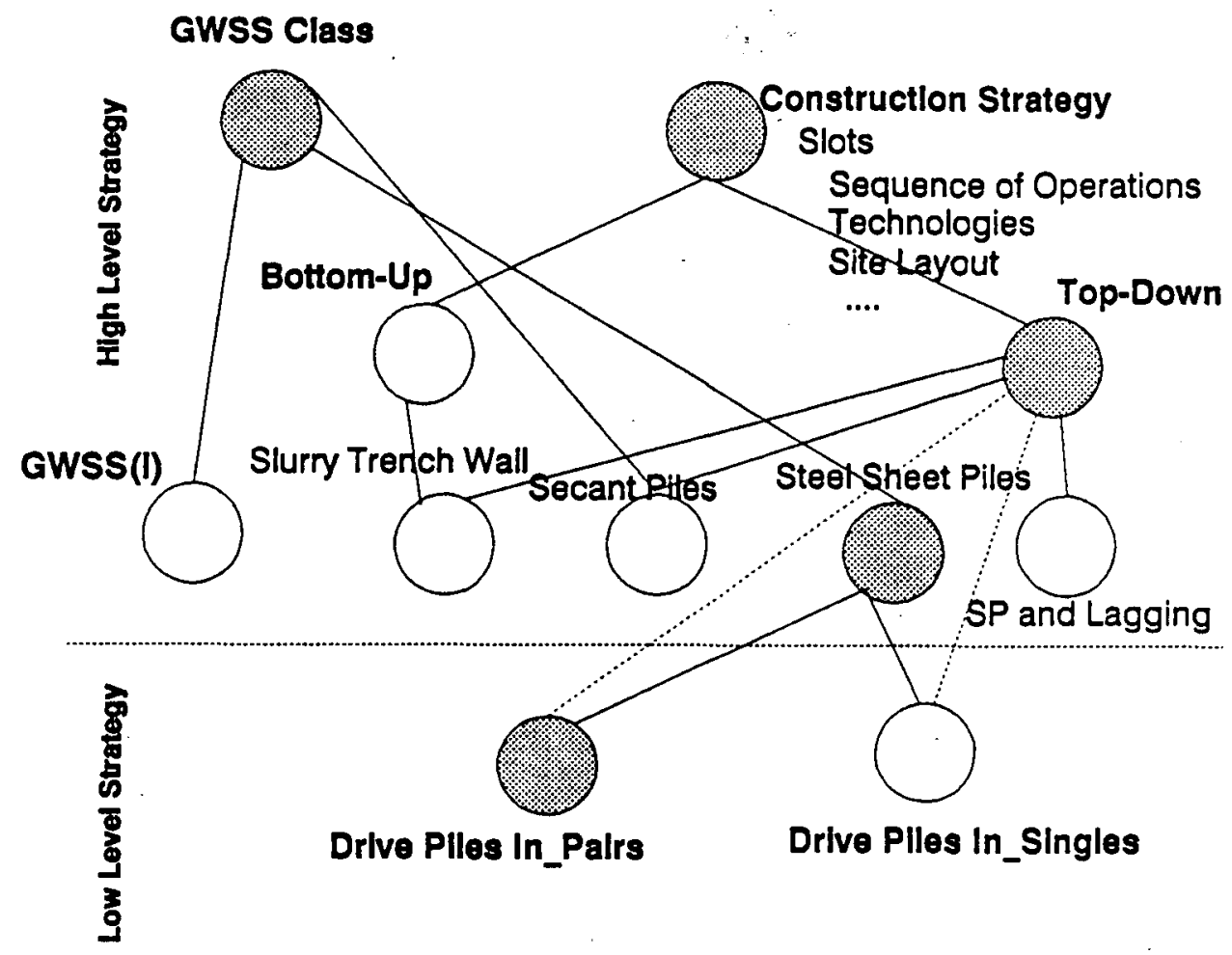

Figure 5.21 Construction strategy Class Hierarchy

Figure 5.22 shows, at the top level, a CPM element with representative slots. class subobjects (model building elements) include crews, equipment, layout, etc. One, or a construction of more than one, of these entities emulates a systematic representation of operations, which in turn yields quantitative/qualitative performance measures.

For CMSA, it is assumed that qualitative variables are dealt with prior to using the process model to determine quantifiable performance. Thus, a subset of resource attributes need to be accessible to the process modelling phase. For instance, at the pile driving activity level, 
three object instances, from two resource classes (hammers and piles), and the soil profile, have to be bound in the process model.

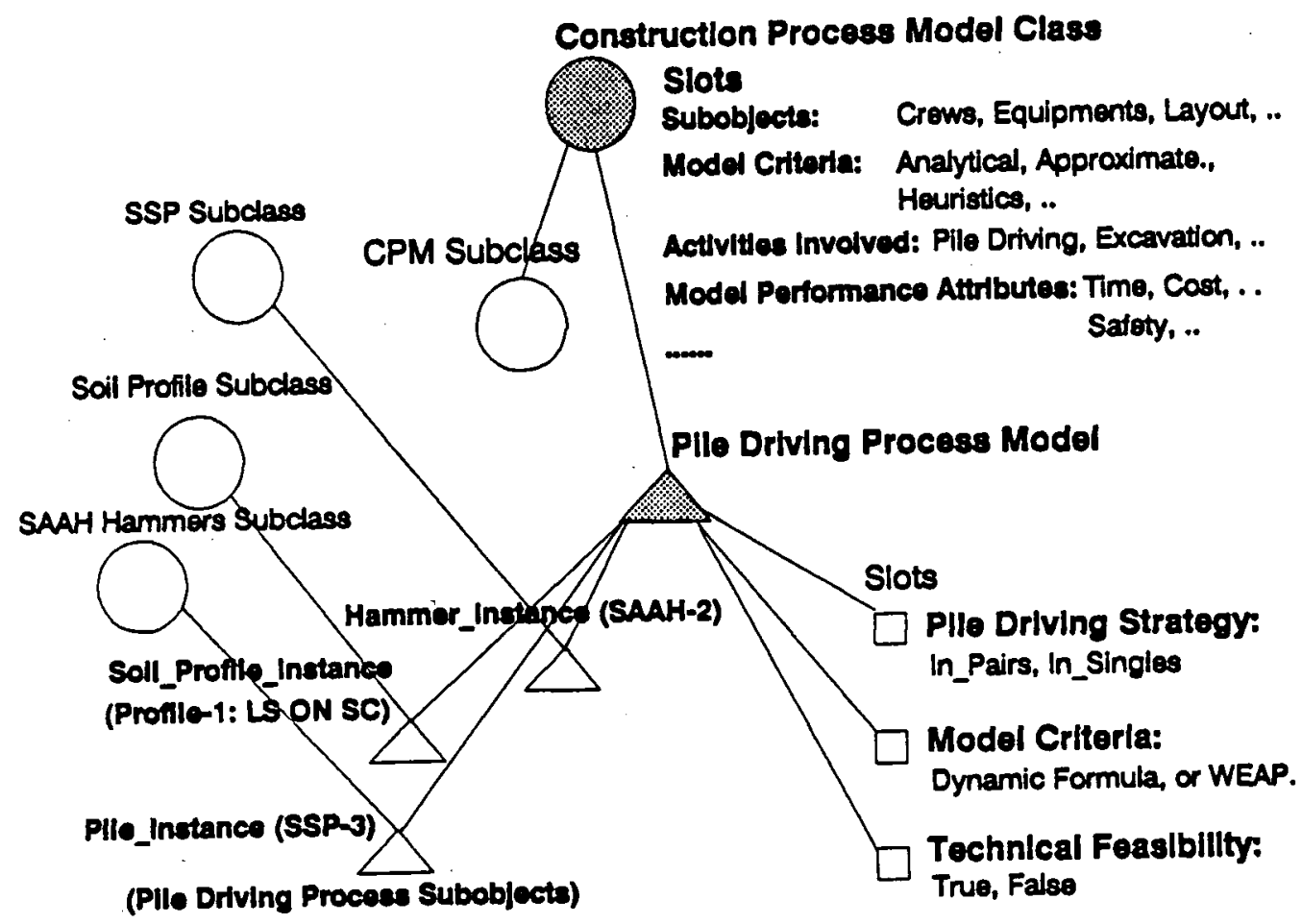

Class, Subclass

$\triangle$ Object, Subobject

Slot

Figure 5.22 Construction Process Model Class Hierarchy

At the process model level, the slots treat pile driving strategy, process model type and technical feasibility. 
Process model type deals with the type of analytic or numerical algorithm used.

In CMSA, for pile driving, the solution model used is based on dynamic formulas which combine the hammer and soil properties to provide an approximate solution.

The third slot is of the technical feasibility state which has a boolean value of either true or false.

\subsubsection{Technical Feasibility Part}

Given the selection of a pile type, a hammer and a pile driving strategy, the next step is to check the feasibility of the combination using the construction process model. This check involves assessing technical feasibility and predicting time and cost performance. Technical feasibility involves determining if the pile can be driven to refusal without damaging it and whether or not the rate of driving can satisfy production rate constraints or targets. The discussion below is given in the context of the PREDICT and ANALYZE Operators of section 4.3.4.

1. Predict: 'The "Predict" operator selects the appropriate construction process model for predicting the method performance after "Design" has been done. The rule shown in figure 5.23 combines the pre-selected matched sheet pile section and matched hammer properties in order to 
predict pile driving progress rate based on the dynamic formula, as derived in Appendix B.

(@RULE = Single_Pile_Variable_Production_Time @INFCAT =5;

@COMMENTS="This rule computes the variable component of driving a single pile based on Dynamic Formula. Note that effective energy consideration is not included - e.g. hammer efficiency as function of the type of the hammer, and pile group effect on pile driving are not treated.";

(@LHS=

(Name (Tunnel.depth +5) (L))

(Show ("Drive.txt") (@KEEP = FALSE; @WAIT = TRUE;))

(Is (Driving_Conditions) ("Soft"))

(Name("In_Pairs") (Piles_Driving_Pattern))

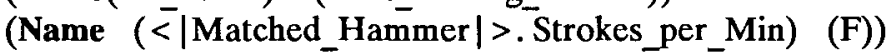

(Name(<|Matched_Hammer $\mid>$.Theor_Energy*Hammer. Total_Efficiency) (E))

(Name(<|Matched_SSP $\mid>$.Surface_Área*2) (SA))

(Name $(<\mid$ Matched_SSP $\mid>$.Cross_Section_Area * 2) (Ap))

)

(@HYPO= SP_Production)

(@RHS=

(Write ("Hammer.nxp") （@TYPE=NXP; @FILL=NEW;

@ATOMS = L,F,E,SA,Hammer_Type;

))

(Write ("soil.nxp") (@TYPE=NXP; @FILL=NEW;

@ATOMS=Soil_Type_1, \Start_1,Finish_1,Soil_Type_2, Start_2,Finish_2; \))

(Execute("Drive.exe") (@TYPE=EXE;@WĀIT=T̄RUE;))

(Retrieve("out.nxp") (@TYPE=NXP; @FILL=ADD; @FWRD=TRUE;

@CREATE=|Var_Time $|;| @ A T O M S=$ Variable_Time.amount;))

(Retrieve ("out.nxp") (@TYPE=NXP; @FĪL=ADD; @FWRD=TRUE; @CREATE=|Feasibility $\mid ; \backslash @ A T O M S=$ Technical_Feasibility.State;))

(Do (Check_Feasibility) (Check_Feasibility))))

Figure 5.23 Technical Feasibility Rule

\section{Left Hand side}

The rule shown in figure 5.23 starts with the depth of the tunnel, or excavation, from the context information (tunnel depth) and adds 5 feet to it, as a default, to determine the 
pile length. The extra length is to minimize, if not prevent, soil boiling or heave. The second condition displays a text file, "Drive.txt", which briefs the user on the questions to be asked by the system, what this rule will do and what is expected to happen after firing this rule. The third condition checks that the driving conditions correspond to soft, as inferred from the strategy component based on soil conditions. The next condition assigns a string value of "In_Pairs" for the Pile_Driving_Pattern strategy variable. Conditions four and five, assign the "Matched_Hammer" frequency and theoretical energy to variables $F$ and E. Condition six assigns the Matched_SSP surface and cross section areas to variables $S A$ and Ap. These variables are used as part of the input for dynamic formula routine. The hypothesis, SP_Production, refers to whether or not the sheet pile production rate is acceptable (True or False).

\section{Right Hand side}

The input required for pile driving routine is written in two files (*.nxp format) using the "Write" operator. The first one is "hammer.nxp", which includes hammer and pile data, and the second is "soil.nxp", which includes the soil profile input. 
The "Execute" operator calls the executable file, "Drive.exe", and runs it in the DOS environment. "Drive.exe" is a compiled program for predicting the speed of pile driving given a soil/hammer/pile combination. It fails if a constraint such as the allowable number of blows per foot run is violated. The "Retrieve" operator retrieves the database file "Out.nxp", which contains a summary of running "Drive.exe" results. More detailed output is contained in the file "Out.out". The "Retrieve" operator returns two values. The first is the variable time for pile driving, which indicates the speed of driving, and which can be tested against the pile driving productivity. The second variable is the state of technical feasibility, which is True if the pile driving operation is successful, otherwise it is False if the pile is not driven to its refusal or the damageability bound has been violated.

The next hypothesis, "Do Check Feasibility", involves diagnosing the cause of a False response for technical feasibility. For example, a driven "In_Pairs" construction strategy is infeasible because the production rate is too low, or the maximum number of blows before damage will occur is exceeded. 


\section{Analyze (Diagnose):}

Here, we examine how the CMSA can diagnose a failure of a technical feasibility test and suggest a remedy. Consider the hypothetical example of a soil profile of loose sand on stiff clay (given a high loose sand/stiff clay depth ratio) which implies soft driving conditions which in turn suggests driving in pairs. Suppose that the state of technical feasibility returns the value False. Thus:

The "In Pairs" pile driving strategy may be infeasible and pile driving should be done In_singles, even though the soil scenario suggested soft driving conditions. (Note that the pile driving strategy also affects the rate of production as well as the fixed time for pile driving.) The rationale behind this is that when piles are driven "In_singles", soil resistance, mainly skin friction and secondary end bearing, will be decreased by half.

- The number of blows per foot exceeds the allowable limit which indicates high soil resistance, or insufficient hammer energy. One way to remedy this situation is to pick a larger hammer. However, the hammer selection criterion already considered picking the largest hammer that satisfied the steel sheet pile constraints. Therefore, if a bigger hammer is to be chosen, then the strength of the sheet pile must be increased. In other words, a heavier steel sheet pile section is required.

- The production rate did meet the required progress rate variable plus fixed time. As a result driving "In_singles", and/or use of a larger hammer and pile section may be suggested. 
A text file of "Tech_Fea.txt" is displayed to the user to explain this rule, as shown in figure 5.24 , along with the expected actions. If the technical feasibility condition is true, then the "Do" operator invokes the hypothesis of Compute_Production_of_SSP in order to calculate pile driving costs.

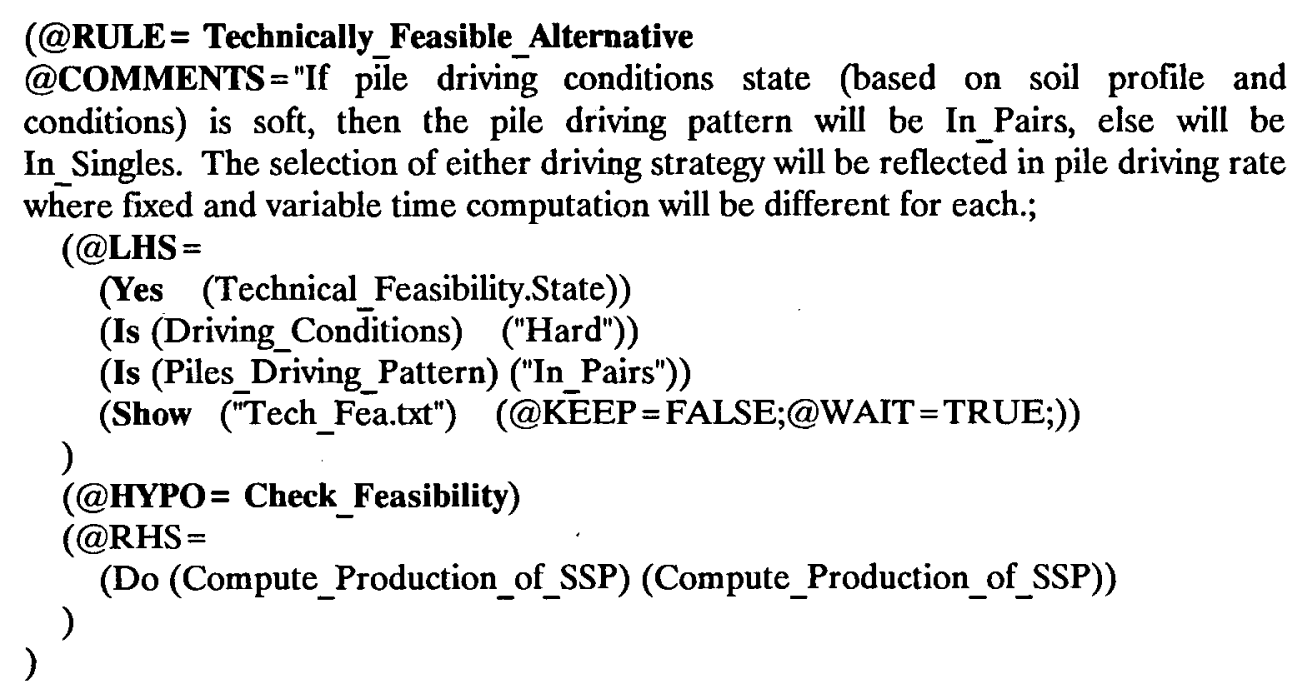
conditions) is soft, then the pile driving pattern will be In_Pairs, else will be In Singles. The selection of either driving strategy will be reflected in pile driving rate where fixed and variable time computation will be different for each.;

(@LHS =

(Yes (Technical Feasibility.State))

(Is (Driving_Conditions) ("Hard"))

(Is (Piles_Driving_Pattern) ("In_Pairs")) )

(Show ("Tech_Fea.txt") (@KEEP=FALSE;@WAIT =TRUE;))

(@HYPO= Check_Feasibility)

$(@$ RHS = )

(Do (Compute_Production_of_SSP) (Compute_Production_of_SSP)) )

Figure 5.24 Method Technical Feasibility is True

A rule directed at reversing the technical feasibility condition from False to True by changing the pile driving strategy from "In_Pairs" to "In_Singles" is shown in figure 5.25 . 


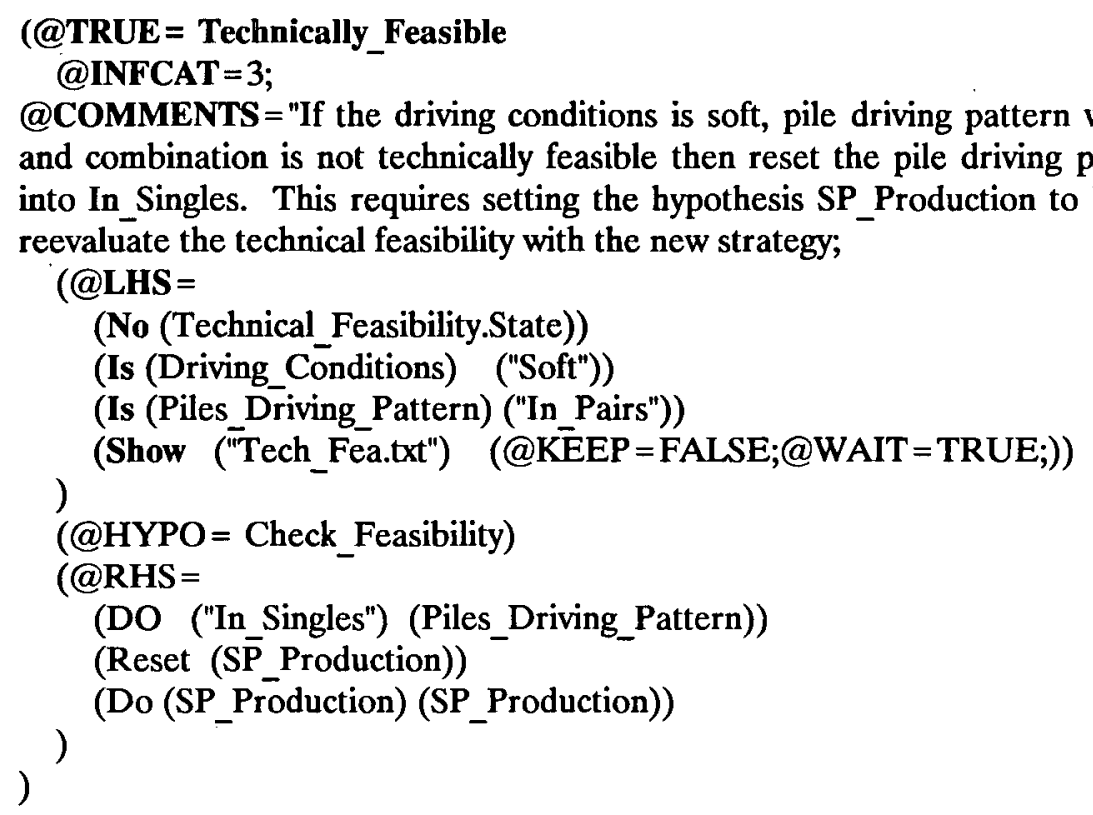

\section{Figure 5.25 Technical Feasibility Diagnostic Rule}

The first condition checks to see if the state of technical feasibility is False in order to perform a diagnosis. The second condition ensures that the driving condition is "soft" which commonly suggests the use of the "In_Pairs" driving strategy. The third condition explicitly checks the variable "Pile_Driving_conditions" value. The "Show" operator displays the text file of "Tech_Fea.txt" to explain the process to the user.

The hypothesis "Check_Feasibility" is the same as the previous one with a different rule (in NExpert, a hypothesis embraces one or more rules). In order to fire the first diagnostic rule, its inference category (eINFCAT shown in figure 5.25) is set to three which is NExpert's priority 
mechanism that fires rules within the same hypothesis in ascending order of their inference category.

To continue, assume this rule's conditions were satisfied and that the system control strategy altered the pile driving strategy from pile driving "In_pairs" to pile driving "In_singles". Then, the "Reset" operator resets the SP_Production hypothesis to Unknown such that control strategy backtracks and re-runs the "Drive.c" numerical routine with changed input variables. The "Do" operator then forces the re-evaluation of the Unknown hypothesis.

\subsubsection{CMSA Chaining and Reasoning (Control strategy)}

In this section, we explain the CMSA chaining and reasoning features for each major operator: Design, Predict and Analyze.

Currently, CMSA is implemented using backward chaining in the NExpert object sense of chaining definitions. Its solution propagates in a forward reasoning fashion. NExpert, originally a rule based system, is a hybrid system which makes it non-trivial to rationalize its chaining and reasoning approach with conventional terminology. For instance, technical feasibility, covered in section 5.3.4, employs a trial and error procedure for the Design/Predict/Analysis cycle in order to modify a design attribute value, i.e. a design alternative, construction 
resource, or construction strategy. This facility is labelled as non-monotonic reasoning in NExpert object, i.e., making assumptions and retractions.

What follows is a description of the chaining and reasoning process in CMSA. Figure 5.26 shows a CMSA network of hypotheses -- rules grouped in categories and interconnected for chaining and inference propagation purposes. Shown in this figure are control strategy clauses used in the methods synthesis process. CMSA accomplishes the control strategy task using the RHS "Do" operator after an hypothesis has proved to be true.

The Do operator performs two operations. It triggers a specific knowledge evaluation and then passes control to the next operation. For example, the following clause from the Design box of figure 5.26,

Do (Select_Pile_Driver) (select_Pile_Driver)

is extracted from figure 5.20, and is interpreted as fire the (Select_Pile_Driver) hypothesis. It is triggered if the previous control clause, extracted from figure 5.12,

Do (select_sSP) (Select_SSP) 
is successfully fired -- i.e. select steel sheet pile. Once the pile driver selection is done successfully, then control passes to applying the hammer dynamic formula embedded in the hypothesis (SP_Production), i.e. predict a productivity rate and check if the pile reaches its refusal depth. The clause that triggers this hypothesis is found in the Predict box in figure 5.26 , i.e.

\section{Do (SP_production) (SP_Production)}

CMSA starts with the suggest operator which currently is a surrogate for the preliminary feasibility knowledge base. The user is prompted with a choice of GWSS alternatives.

The Design task follows risk evaluation if the level of risk is acceptable. The design element subtasks exhibit forward reasoning and chaining modes. The hammer selection task is more complicated. The hammer type is based on soil profile. Hammer size is based on compatibility conditions with the design elements of steel sheet and soldier piles and goal requirements, (e.g. production rate), although it is assumed with CMSA that the maximum energy hammer will be the default choice. Thus hammer selection involves mixed modes of chaining with a forward mode of reasoning. By contrast, "RI" an expert system that configures VAX computer systems, exhibits forward chaining with backward reasoning (McDermott 1984). 


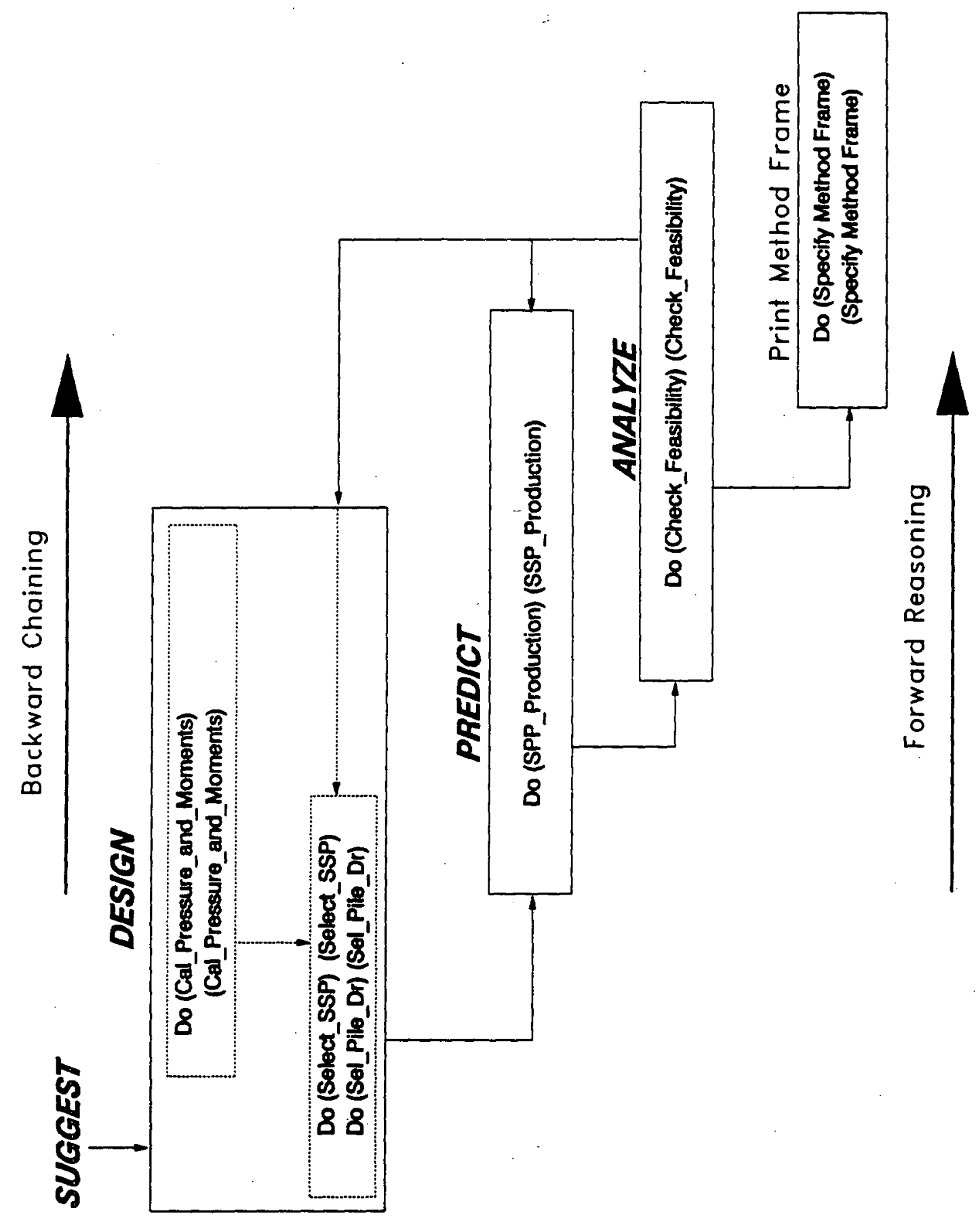

Figure 5.26 CXsA Model of Chaining and Reasoning 
Once operator method attributes are generated, the Predict operator selects a suitable method (procedural routines) for measuring the method performance for the given project context. Next, the Analyze operator applies analysis and/or interpretation routines to the results from the Predict operator. This, in general involves backward modes of chaining and reasoning.

During the Predict/Analyze process, alternative ranking is based on minimum costs. Further, the Analyze operator may exhibit non-monotonic reasoning by making and retracting assumptions. For instance, if the pile driving construction strategy state of In_Pairs is "True", but the method did not satisfy a goal criterion, (say production rate), or method elements compatibility resulted in a failed solution, the original plan could be altered by the CMSA control structure by retracting the pile driving strategy state of In_Pairs to be "False" and assessing the value "True" to the In_Singles state value. Thus, a mix forward mode of chaining and reasoning are used for the Analyze operator. 


\section{The Prototype Example}

\subsection{Introduction}

Features of the prototype CMSA implemented are described in this chapter. Input/output data, solution strategy processing, sensitivity of decisions to input changes, and the explanation facility are described first. An example problem is demonstrated using a step by step approach. The example consists of two cut-and-Cover shoring methods in parallel -- steel sheet piles and soldier piles and lagging. The second part of this chapter is devoted to a detailed example of the risk assessment process, implemented in NExpert object as an independent module.

\subsection{Example Problem Description}

The example problem is a proposed tunnel, $1000 \mathrm{ft}$ long, 60 ft deep and $20 \mathrm{ft}$ wide. The soil profile consists of two layers: a $40 \mathrm{ft}$ top layer of loose sand, and stiff clay below that. The contract duration for this project is estimated to be a maximum of 240 days (or $5 \mathrm{ft} /$ day), with a unit cost of $\$ 2,800$ per foot $\pm \$ 500$. It is assumed that upper and lower bounds for unit costs and production rates are given for the Cut-and-Cover tunnel alternatives.

A record of a session is provided, with user input and CMSA system responses. Example screens are shown as 
appropriate. Due to the lack of NExpert's explanation facilities, explanation files with an extension "txt" were used extensively to explain some of the CMSA operations and query processes to the user.

\subsubsection{Session start}

A session commences by suggesting either a "datum" for input variables or suggesting a NExpert "hypothesis" as shown in screen 6.1. This window is invoked by the command "Suggest" followed by the command "Knowcess" from the Expert Command Menu. From this window, any hypothesis could be highlighted and put in the suggest/Keep corner before NExpert starts the session, e.g., the hypothesis "Select_A_GWSS" is used to trigger the CMSA session. Also this window is used to list any datum (a premise in a rule) and triggers the beginning of the session by first evaluating the premise (as opposed to the hypothesis). Control is then passed to evaluate the rule. Once a hypothesis or datum is placed in the Suggest/Keep corner, "OK Knowcess" command is selected, the session starts. 


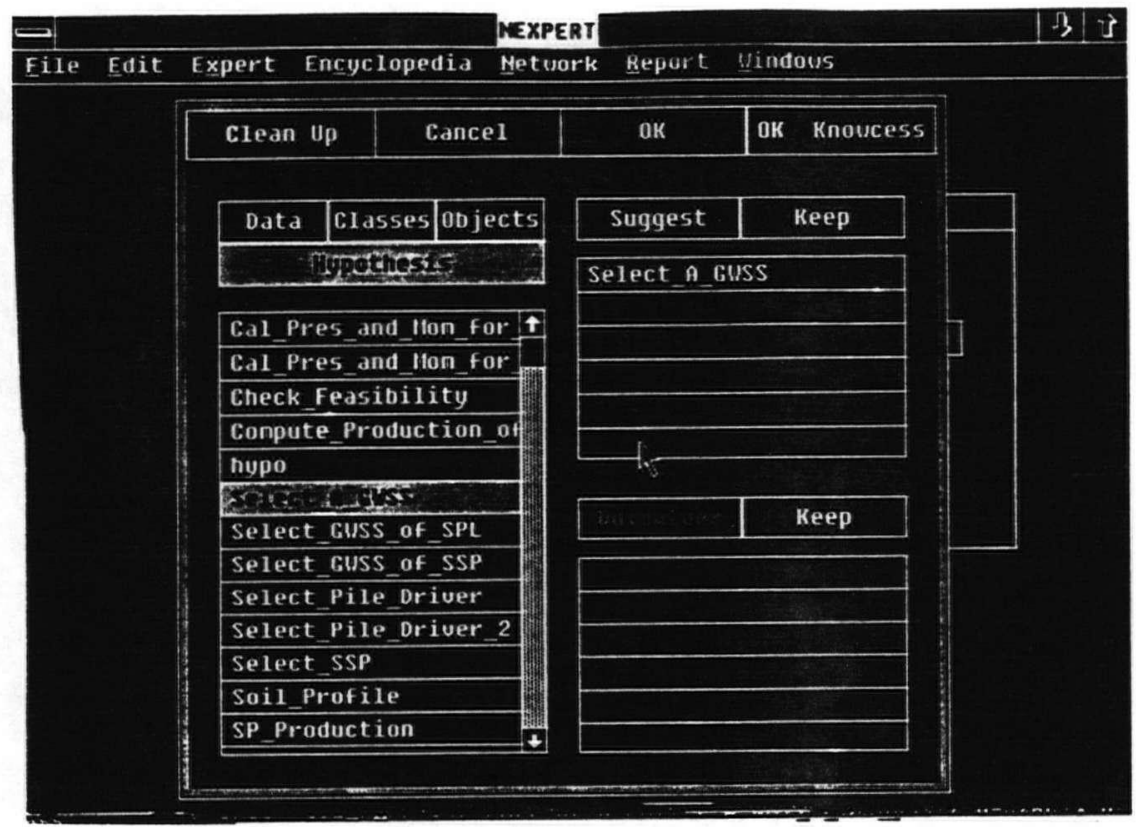

Screen 6.1 Rnowcess Hypothesis Command Menu

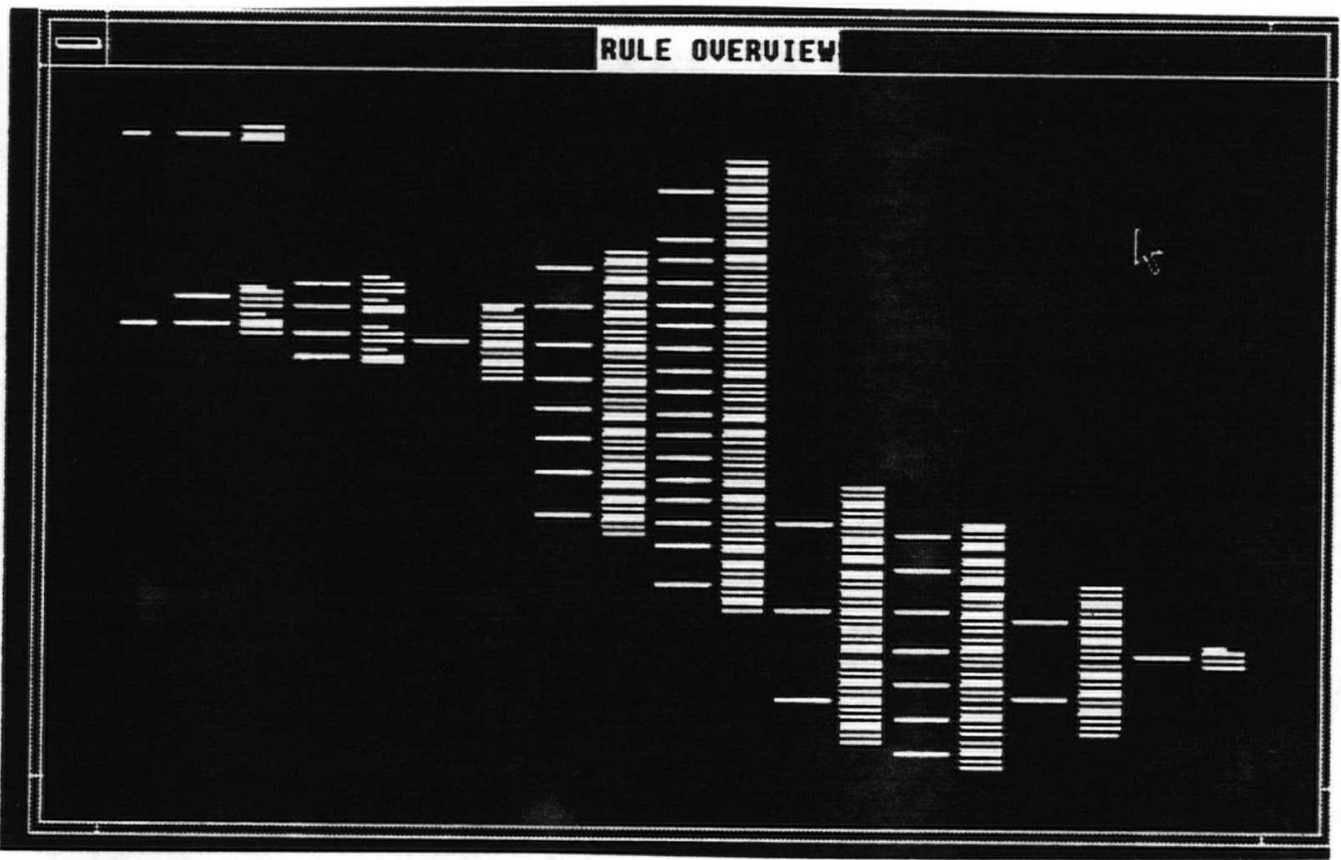

screen 6.2 CMSA Overview Rule Network Window 


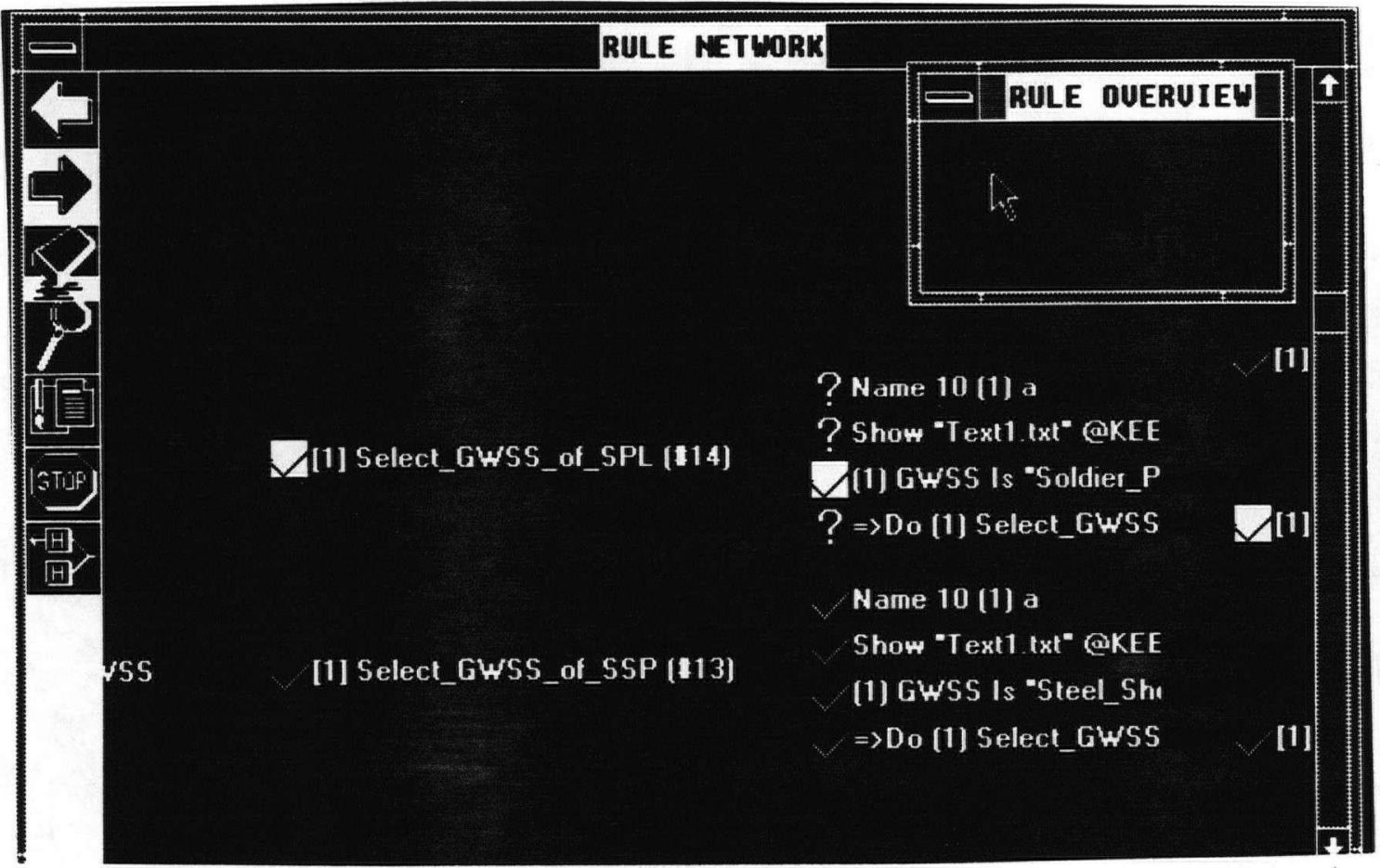

screen 6.3 CMsA Rule Network Window

After firing "Select_A_GWSS", a text file (CMSA.txt) appears on the screen, introducing the user to the Cut-andCover tunnelling problem. Thus, CMSA is set in a "backwardchaining" mode. For the loaded CMSA knowledge base, screen 6.2 shows the overall rule network using the Overview Rule Network (ORN) window. The highlighted branches represent rules that are fired successfully during the session. NExpert Command Menus are shown (File, Edit, Expert, etc.) in the NExpert Environment Screen.

By zooming in on the dotted box of ORN, the "Rule Network Window" focuses visually on a individual rule or a group of rules in the CMSA knowledge base, as shown in screen 6.3 . The Rule Network Window (RNW) is enlarged to the size of the 
screen. In the upper right hand side of the slide, the Rule Network Overview (RNO) is shown. In the middle of the RNW, the rule "Select_GWSS_of_SSP" is shown fired (a condition or hypothesis is indicated by the following icons: "True" by a check mark, "False" by a highlighted check mark, "Unknown" by a question mark, "Not Known" by an empty box, "Being Currently Investigated:" by a target, and "Being Evoked:" by an asterisk.

Continuing with the session, CMSA provides the user with the following potential feasible alternatives as shown in screen 6.4 .

$>$ select A GWss ?

1. Steel sheet Pile (SSP).

2. Soldier Pile and Lagging (SPL).

The system asks the user to choose one GWSS alternative to start the detailed $\mathrm{KB}$ part. For example purposes, the two alternatives of steel sheet Piles and soldier Piles and Lagging are assumed to have survived the preliminary screening process.

Assume the user chooses the steel sheet pile alternative. The next part of the session involves specifying the soil context. 


\subsubsection{Problem Context specification}

\section{What is the Number of Soil_Layers?}

1. One

2. Two

\section{>> Two}

The user is prompted for either a single soil layer or a two soil layer scenario. The latter has been selected as shown in screen 6.5 .

CMSA asks for the soil type for the top layer as shown in screen 6.6. Alternatively, a second input format is based on a standard Penetration Test (SPT) Profile.

If the first input format is adopted ${ }^{11}$, assume you select Loose_Sand as the top layer. Screen 6.7 shows the input for the 40 foot loose sand layer depth. Screen 6.8 shows that stiff clay is chosen as the second soil layer. Screen 6.9 shows the water level input.

other queries include the tunnel depth in feet (60), and the tunnel length in feet (1000).

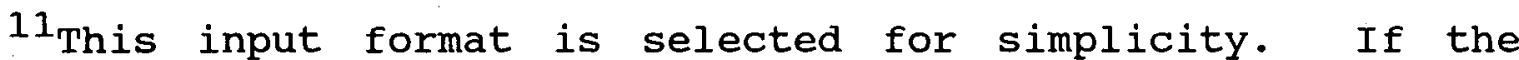
input for the SPT profile is selected, the user will be required to input SPT readings at 5 foot intervals for the depth of the tunnel or excavation. 


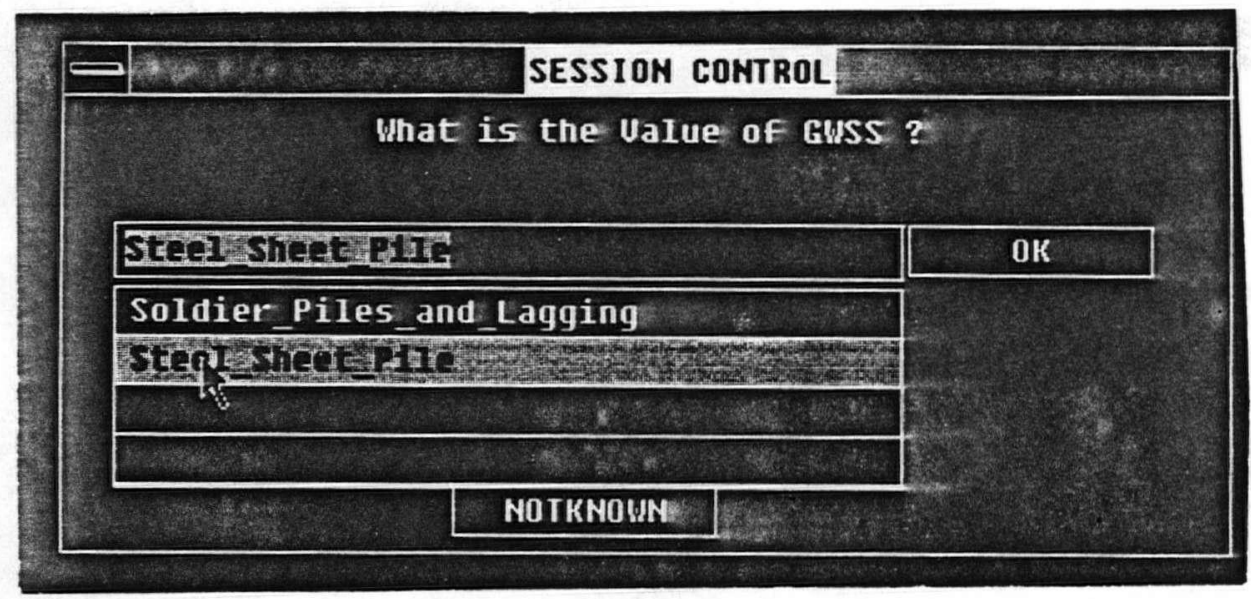

screen 6.4 Gw8s Feasible Alternatives

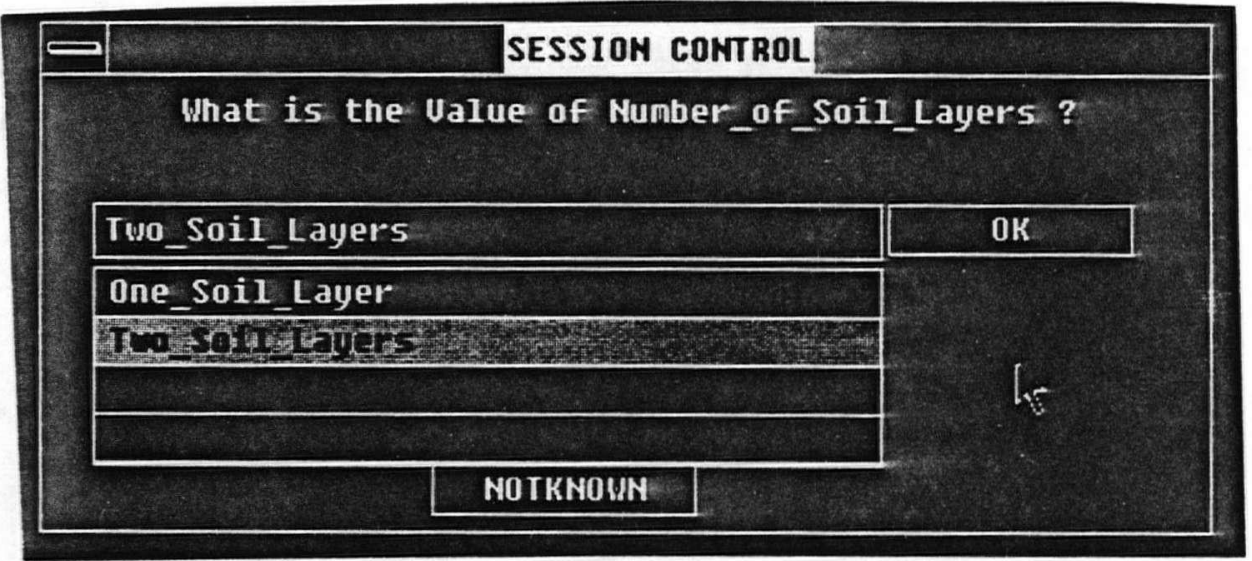

screen 6.5 soil profile specification (1)

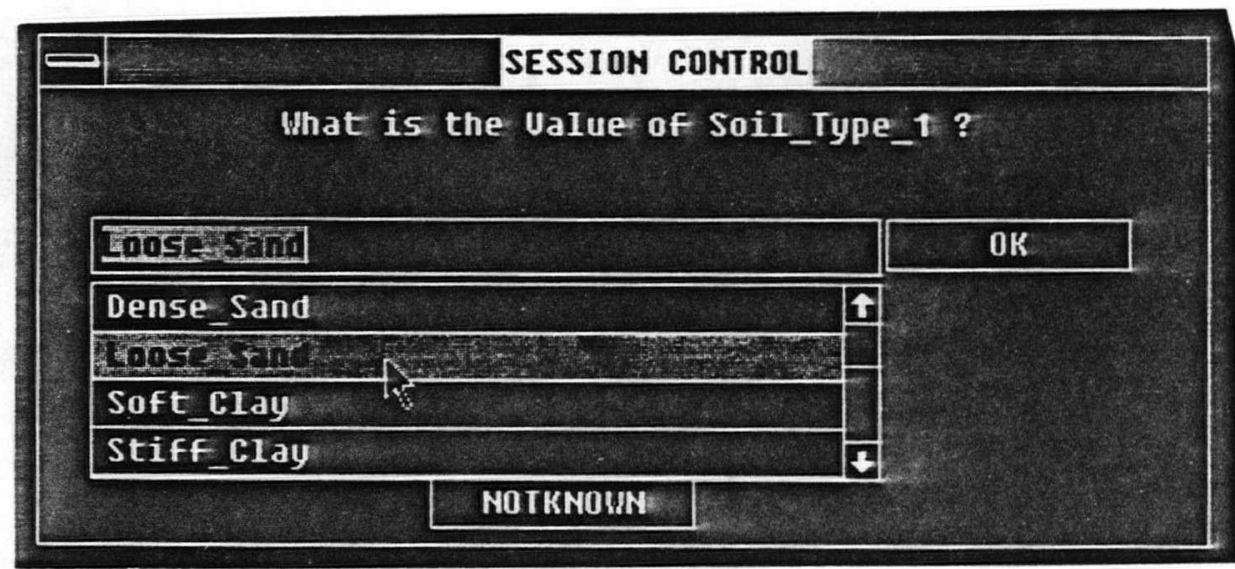

Screen 6.6 Soil profile specification (2) 


\section{$\Longrightarrow$ SESSION CONTROL}

What is the depth of Layer_1_of_Soil_Type_1?

41

screen 6.7 soil Profile specification (3)

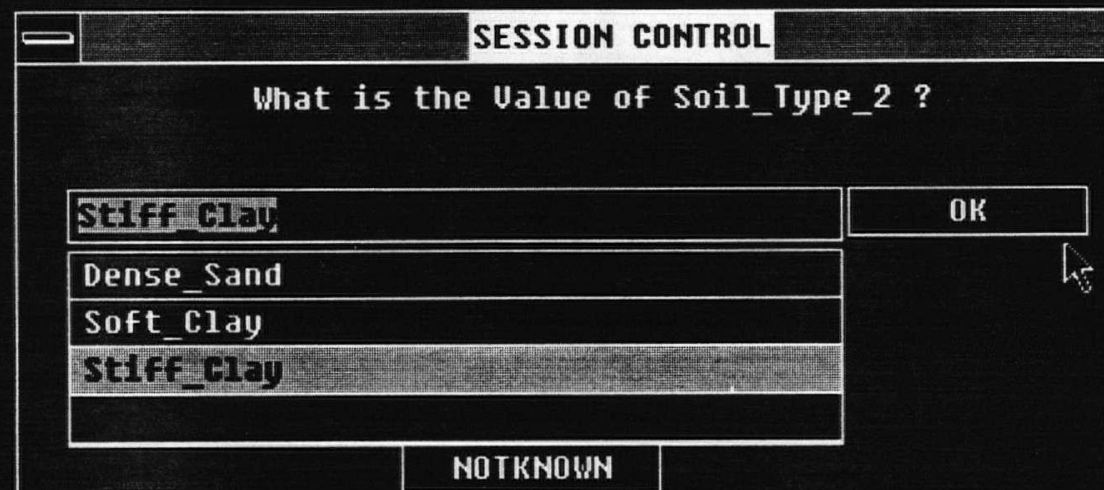

Screen 6.8 Soil Profile Specification (4)

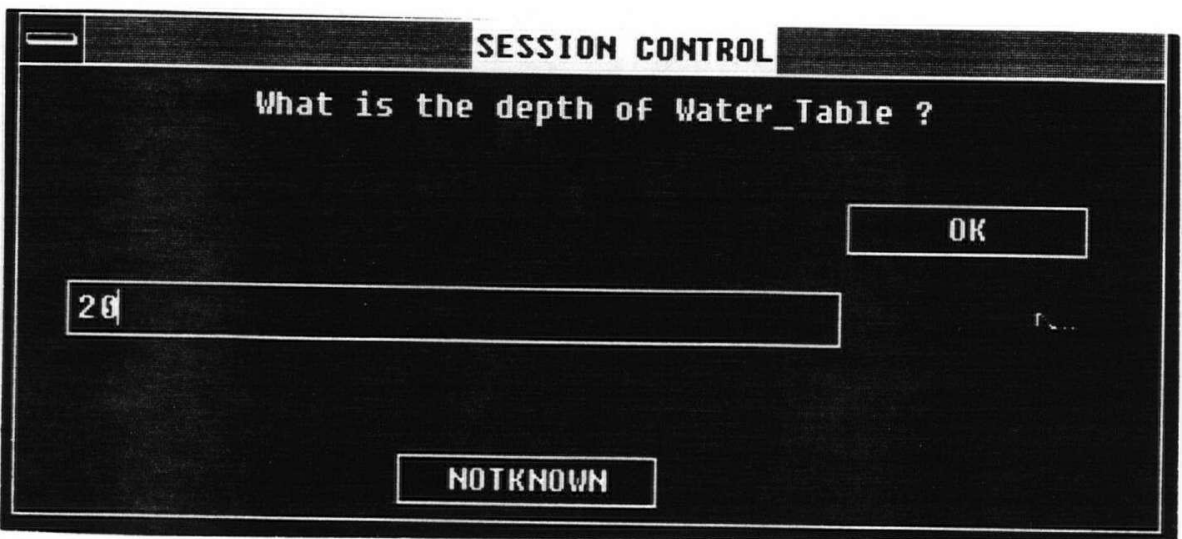

Screen 6.9 Water Table Level Input 
Gwss Technical Feasibility Assessment: The CMSA System then processes the following operations:

1. Calculate pressure and moments ${ }^{12}$ : A rule is used to do the computations for this scenario; see Appendix A for the computation procedures. The hypothesis "Calculate Pressures and Moments for "Two_Soil_Layers Scenario" is fired.

2. Select a suitable sheet pile if it exists with the given data base of "ssp.nxp" (see screen 6.10). The following operations are carried out by CMSA.

- Retrieve steel sheet Pile database "ssp.nxp";

- Fire section Modulus Rule which incorporates moments based on Retaining system Spacing;

- Select ASTM steel sheet Pile section of PZ_27;

- Attach Selected Pz_27 to Matched_SSP;

- Inherit Pz_27 properties to Matched_SSP;

- If this fires successfully, then pass control to select Pile Driver.

${ }^{12}$ Pressure and moments calculations are based on the "default" spacing of the retaining system (15 ft vertically and 12 ft horizontally). 


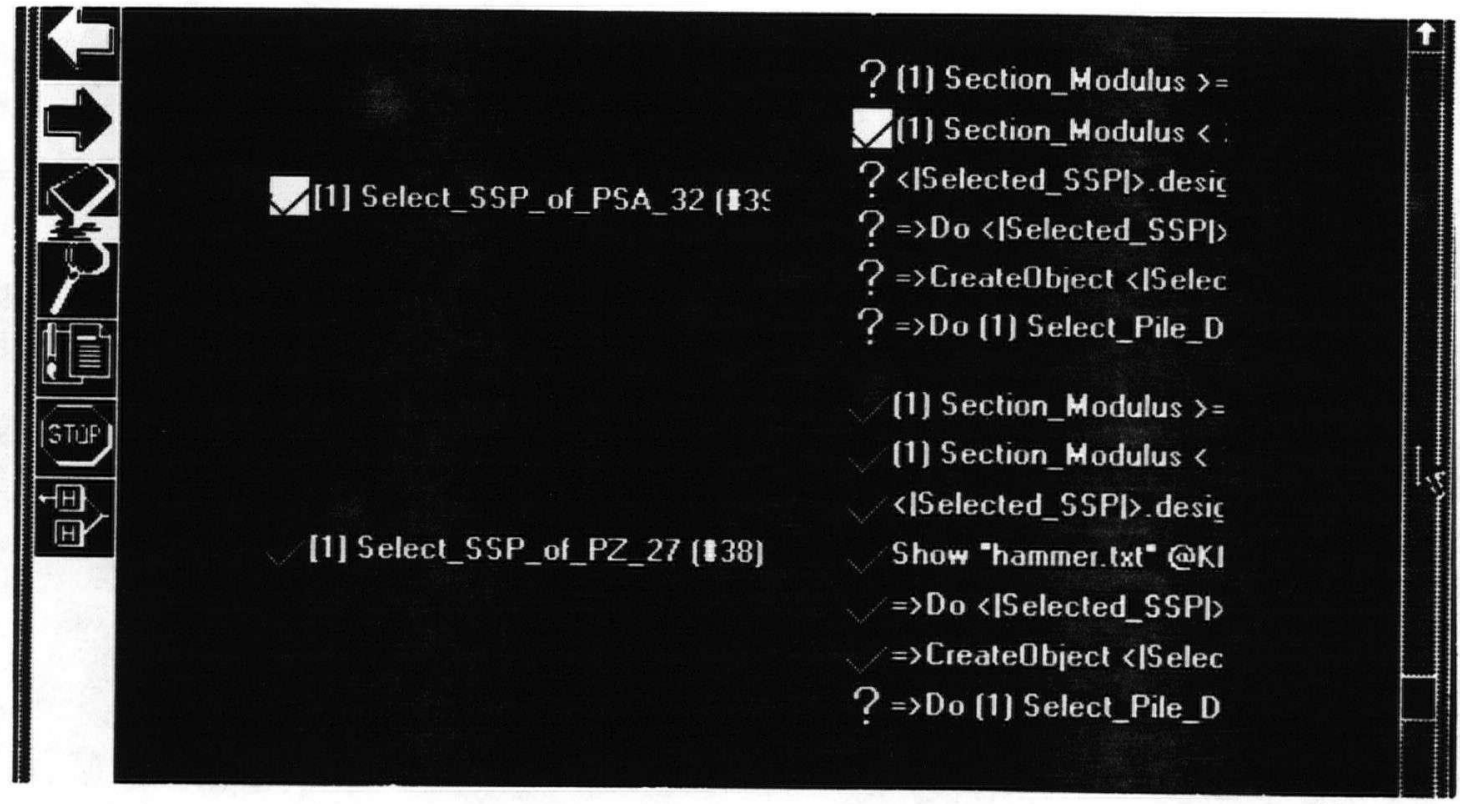

Screen 6.10 Hypothesis "select_suitable_sheet_Pile" is Fired successfully

After successfully selecting a suitable SSP data base (SSP.nxp), control is passed to the "Select a suitable Hammer" hypothesis. After a pile section has been successfully selected, the system selects a suitable hammer as follows. Based upon the soil stratification and sequencing, a generic type of hammer is selected. For this example, the impact hammer type is more suitable than a vibratory one. A Double Acting Air Hammer (DAAH) is found to be an appropriate impact hammer.

Once the type of hammer is specified (the hypothesis "Select Pile Driver" is fired), it is then sized using 
heuristic

rules

(the

hypothesis

"Select_Vibratory_or_DAAH_Hammer_PD" is fired, see screen 6.11). The CMSA proceeds as follows:

- Retrieve DAAH database "DAAH.nxp";

- Based on experiential rules, the DAAH is sized in terms of "Rated Delivered Energy". If the search is not successful and no such size exists within the database, a message appears offering the alternatives of abandoning the search and quitting; selecting another hammer; or selecting another shoring alternative. The default strategy is to pick the hammer with the highest delivered energy;

- If a DAAH hammer of the required size is found, it will be attached to the "Matched_Hammer" object and its properties (model, delivered energy, unit cost, etc) are inherited from the "selected_Hammer" list. cost is not considered as part of the criterion at this stage;

- Control is then passed to the driving strategy knowledge base.

The selected hammers class and dynamic objects created after firing the above rule are shown in screen 6.12 . 


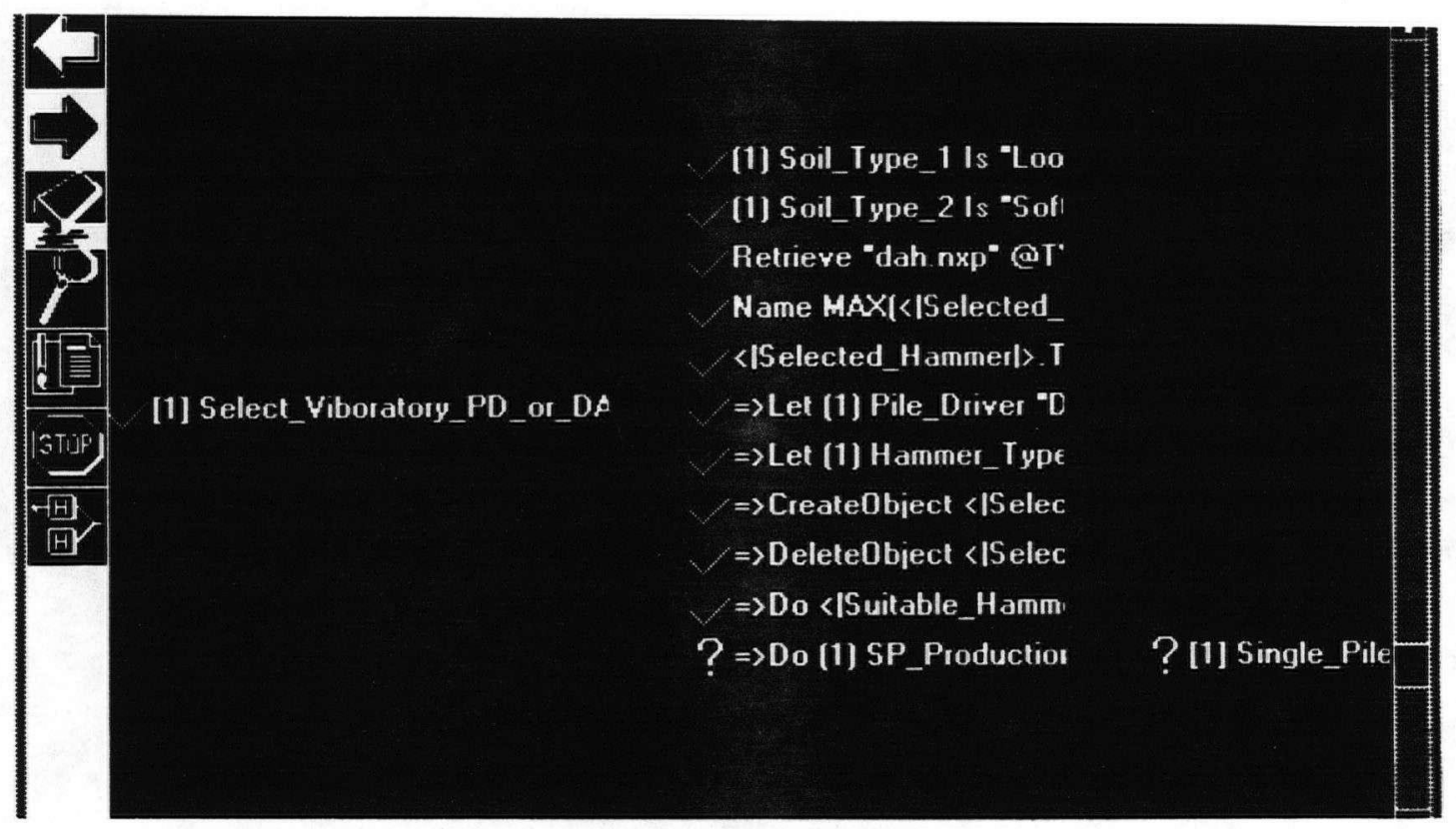

Screen 6.11 Hypothesis "select_suitable_Hammer" is Fired successfuIly

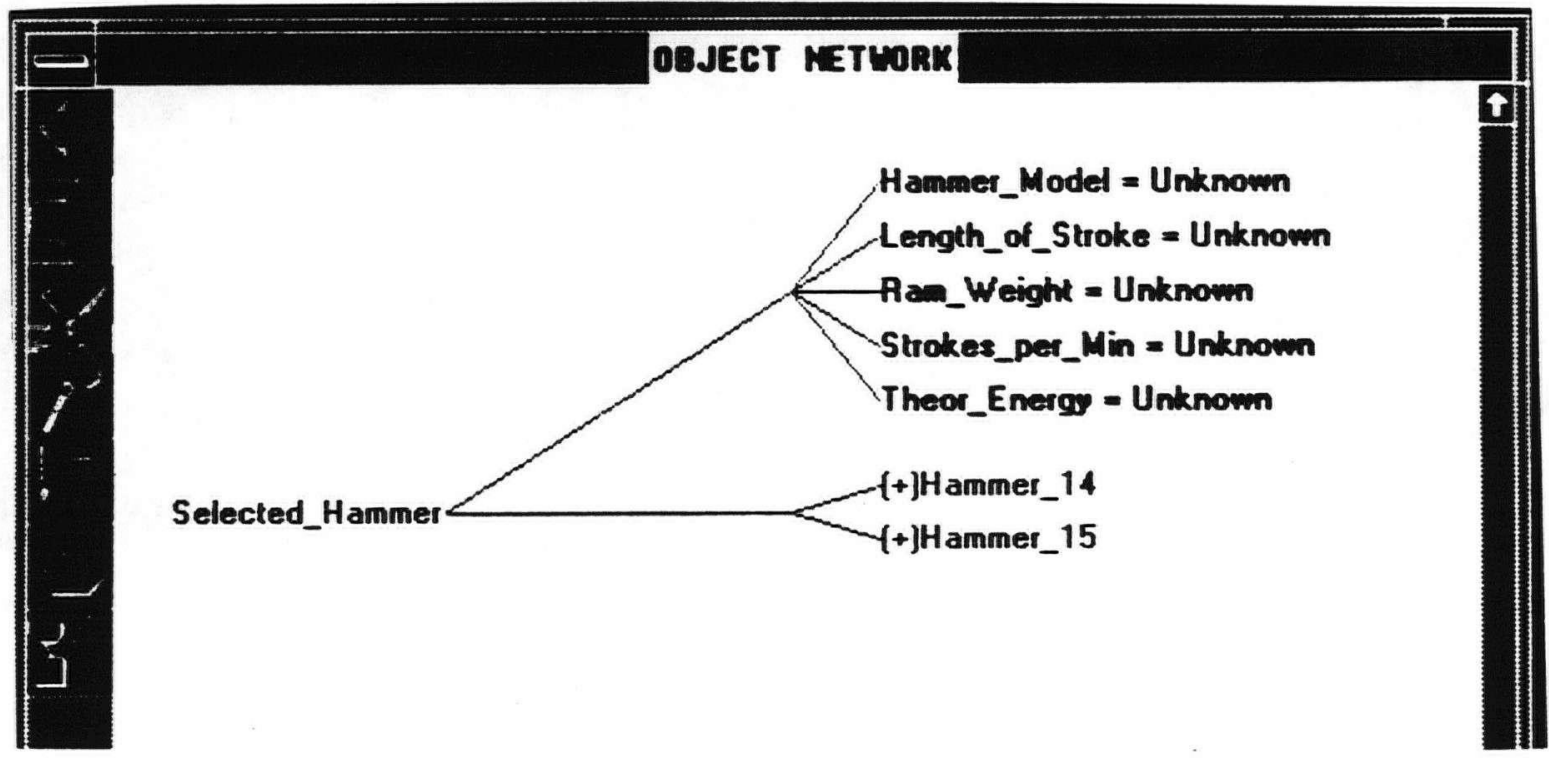

screen 6.12 selected_Hammers Class and its Dynamic Objects 
To predict the production rate for the hammer/pile/soil scenario using the dynamic formula (the hypothesis "SP_Production" is fired), the system next asks the following question.

What is the Efficiency of the Double Acting Air Hammer (DAAH) ?

The user is requested to provide a hammer efficiency estimate (this could be automated if desired). Under different project and soil conditions, and indeed within the same site, the pile driver efficiency may vary.

Assume in place of the CMSA default value, the user has estimated an efficiency of $71 \%$. Screen 6.13 shows the hammer efficiency input by the user.

The pile driving strategy is an important factor which must be determined prior to running the technical feasibility routine (Drive.c routine), where the soil, pile, hammer, and driving strategy are combined. Soil conditions and stratifications determine the state of driving conditions. A "driving strategy text file" is displayed to explain to the user the different state variables of this parameter.

Do you want to choose a driving strategy or leave it to the system? Yes, No.

If yes, then the user has to choose: 


\section{Drive Piles In_Singles, \\ 2. Drive Piles In_Pairs.}

A text file "Drive.txt" is displayed to explain and recommend the use of either strategy. Screen 6.14 shows one page of the Drive.txt file which outlines the assumptions for using each pile driving strategy. For this example, we select "Drive Pile In_Pairs".

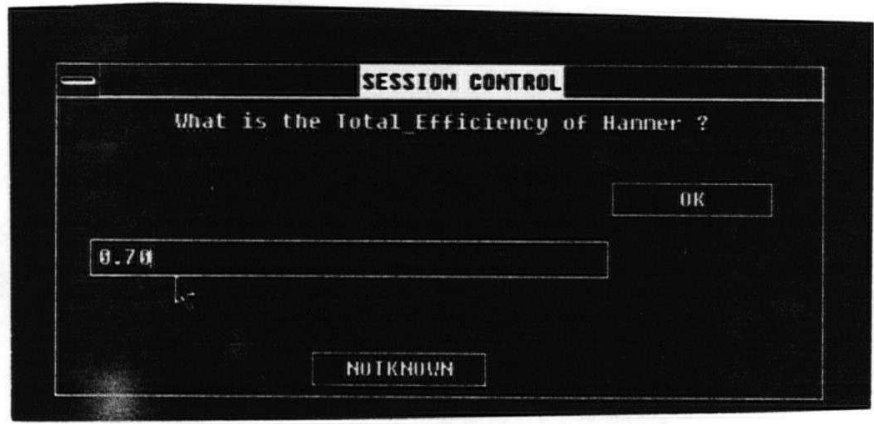

Screen 6.13 Hammer Efficiency Input

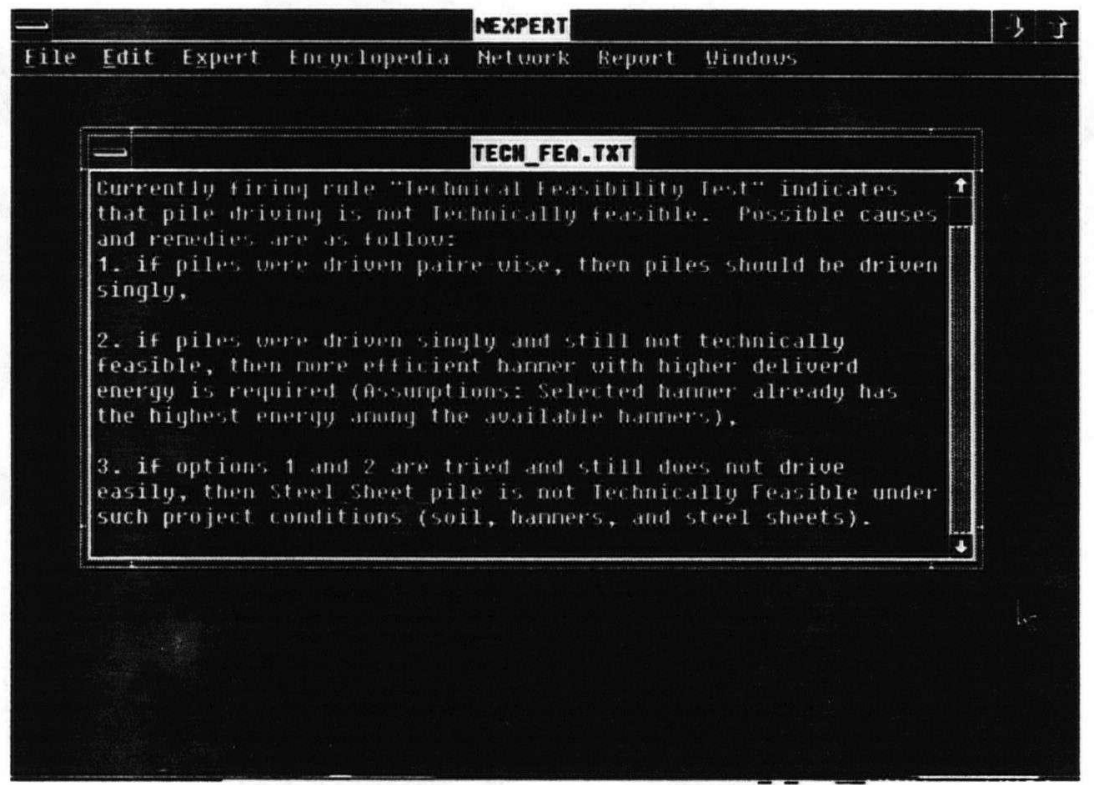

screen 6.14 "Drive.txt" Explanatory File 


\section{> Let CMSA Choose ? "Drive In Pairg"}

- The input for the "Drive.c" routine is written into the files "hammer.nxp" and "soil.nxp". The first file contains information about the hammer properties (hammer type, frequency, rated energy, etc). The second file contains information regarding the standard Penetration Test profile, soil types, their sequences, and thickness.

- The compiled "Drive.c" routine, "Drive.exe", is executed (when the rule "SP Production" is fired, this premise is evaluated) by CMSA (see Appendix B for the derivation, coding, and input and output files for the "Drive.c" routine).

- The output parameters are written into the file "out.nxp" for the NExpert interface, and "out.out", which allows for viewing the output results as a stand alone application.

- NExpert thus checks if the state of Technical Feasibility is "True" or "False", with the text file "Tech_fea.txt" displayed to explain the underlying logic. "True" implies that the combination of the strategy of pile driving, matched sheet piles and matched DAAH hammer satisfy the pile driving constraint (blows/ft $\mu 120$ and the performance measures fall within a required range).

The quantitative output variables from the "Drive.c" routine are: incremental and cumulative number of hammer blows; skin friction; end bearing friction; average set; and speed of pile driving. The qualitative parameter is the technical feasibility state, which would be set to "False" if the threshold of the average set/average speed of pile driving has been violated.

The technical feasibility state is interpreted by CMSA (hypothesis "Check Feasibility" is fired), as "True" for a 
feasible combination of the methods attributes. If the state is found to be "False", then the diagnostic part of the technical feasibility is invoked to offer a plausible method attribute. For instance, if the pile driving strategy was "In_Pairs", then it is possible to change this strategy to driving "In_singles".

The "Drive.c" routine finds the variable time component of the production rate. The total production time per pile, or set of piles, is the sum of the fixed and variable components. The fixed time component is obtained from the user in order to compute the total duration of the pile driving activity, and thus the production rate in terms of piles per day, foot run per day, and total cost.

The following questions are used to perform a quantity take-off and estimate total project duration.

What is the fixed time to position, splice, and seat a pile? (Default 5 minutes)

\section{6 minutes}

what is the standard Length of the steel sheet Piles?

SSPs come in different sizes, including $25 \mathrm{ft}, 40 \mathrm{ft}, 50 \mathrm{ft}$, $70 \mathrm{ft}$; with the CMSA default being $25 \mathrm{ft}$.

$>>\underline{50 \mathrm{ft}}$

Production rate and quantity take-off computations for the steel sheet pile GWSS alternative are then performed. 
The detailed costs of this alternative include material, labor, and equipment.

Next, control is passed to the risk assessment KB. The second part of this chapter presents how risk is quantified in monetary terms. For ease of risk component integration with CMSA and for computation speed, the risk computation has been encoded in a "Risk.c" routine. This routine is fired by CMSA and returns the risk cost fraction, as percentage of total cost, for SSP alternative based on risk categories and probability data input file "SSP_Risk.nxp". The risk fraction is then added to the total cost of the SSP alternative. Selected variables relevant to this alternative are then written to the "Results_1.nxp" file, as shown in screen 6.15 .

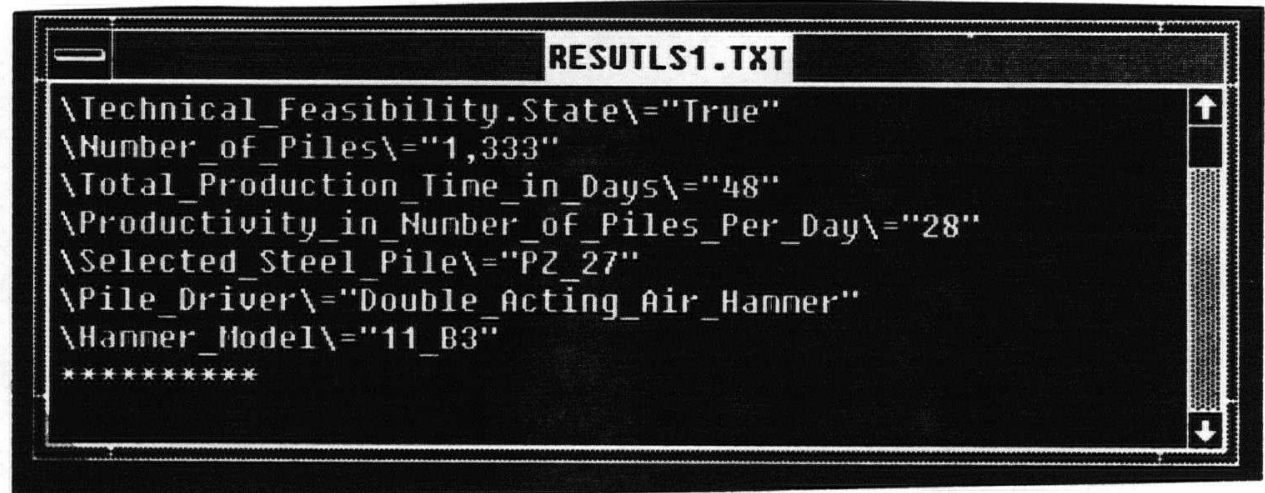

screen 6.15 "Results1.nxp" File

Next, the system considers the second feasible solution of soldier piles and lagging, queries the user in a way 
similar to the first alternative and writes the results to "Results_2.nxp".

Once a preliminary feasible alternative has proved to be feasible at the low level, its attribute values are stored in its results frame, and the criterion of minimum total cost is applied to rank the alternatives. For our example, CMSA selects the steel sheet pile alternative. Based upon other factors, such as production rate, preferred resources, and other attribute values generated in the CMSA session, the user may wish to over-ride this recommendation.

Figure 6.1 displays the context variables (the high level primitives for the represented major concepts) that are instantiated during the CMSA session. The direction of inference shown is from bottom to top. At the bottom, three major input categories are identified: tunnel dimensions, soil profile and conditions, and risk input. The structural members feasibility instantiation is dependent upon site conditions and tunnel dimensions. Risk input data is necessary for assessing the risk component. Once technical feasibility is proven, the session is terminated, and the session results are printed in output files. 


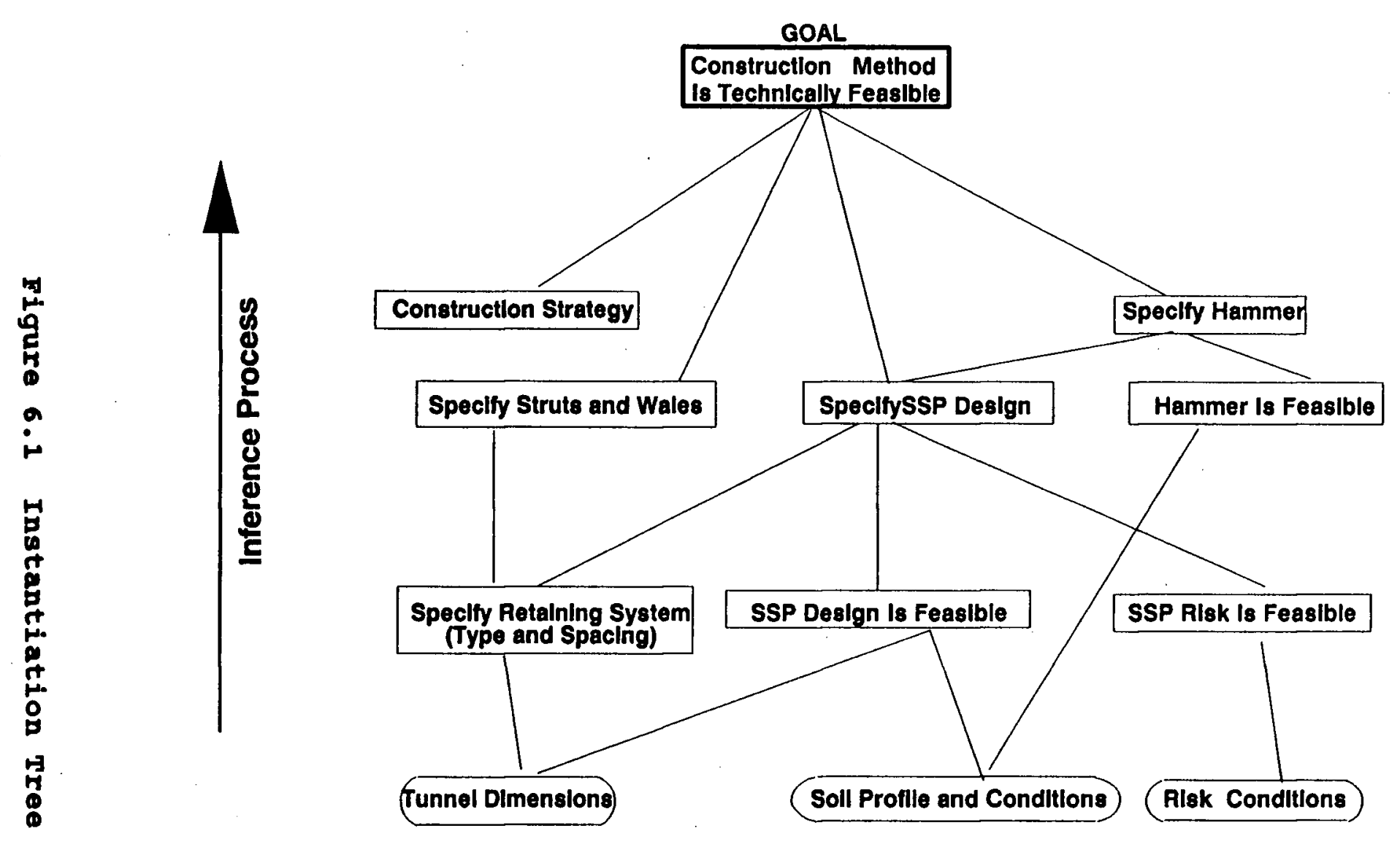

0
0
0
0
0
0
0
0
0
0
0
0
0
0
0
0
0
0
0
0
0
0
0 


\subsubsection{Modified Example}

Results from a second example are presented to demonstrate the ability of the CMSA to respond to a change in input conditions. A variation of the first example is used to illustrate the infeasibility of a GWS alternative in a different project context. For the previous example soil profile, suppose the following variables are changed: the top layer is changed from loose sand to dense sand and the second layer changes from soft clay to stiff clay; and the hammer efficiency is changed from $70 \%$ to $50 \%$. Assume all other project conditions are the same.

The system executes the control strategy and selects a Pz_32 steel sheet pile section and a Vulcan single acting air hammer of model "O" (hammer energy is 24,375 lb-ft per blow).

A $50 \%$ efficiency for the single acting air hammer is then specified. Given the soil stratification nature (dense sand on stiff clay is considered to be a compacted soil), the system recommends using the driving strategy "Drive In singles"

After executing the compiled "Drive.c" routine, "Drive.exe" is executed (the hypothesis "SP_Production" is fired). A text file "Tech_feal.txt" is displayed. This text file explains that the technical feasibility state is "False" and thus the search for a feasible solution has failed. By retrieving the data encyclopedia notebook 
"resultsl.nxp", it is found that pile driving was halted at a depth of $55 \mathrm{ft}$. A possible remedy includes using a larger hammer than the one contained in the existing SAAH knowledge base.

Therefore, for this project context, the CMSA informs the user that it failed because it is not possible to drive the selected pile to its refusal depth. Thus, it declares that the SSP alternative is infeasible at the detailed level.

\subsection{Risk Component Assessment Implemented}

\subsubsection{Introduction}

The risk component was implemented by using NExpert object and by writing an evaluation routine. The NExpert module takes the user through a step by step input data process and displays text files to explain the decision tree. The evaluation routine is used to embed the risk component in CMSA to speed up the session and to minimize user input. Both implementations follow the risk assessment framework as described in section 4.4 .

\subsubsection{NExpert Risk Implementation}

An example session is used for demonstration (see figure 4.12, Risk Assessment Tree). Two shoring alternatives are considered in parallel: Steel sheet Piles (SSP), and Soldier Piles and Lagging (BPL). 
A session commences as follows:

what is the Initial cost for steel sheet piles?

This cost is assumed to be obtained from the detailed feasibility part.

$>\$ 3.00 \mathrm{M}$

What is the Initial cost for the soldier Piles and Lagging ?? $>\$ 2.80 \mathrm{M}$

This cost is assumed to be obtained from the detailed feasibility part.

What is the Likelihood of worse_Than_Expected subsurface Conditions?

$>\mathbf{3 3} \%$

The questions that follow treat the assessment of the risk component for the Worse_Than_Expected scenarios.

The user is first asked if there is a chance of a catastrophic damage risk for this alternative, or if the likelihood of this catastrophic risk exceeds a pre-specified value. If yes, then the alternative will be eliminated without asking further risk related questions. If the answer is no, the computation then takes place for the total risk cost. 
The system starts with the computation of the worse_Than_Expected risk branch. If the risk costs associated with this branch are bounded, then the alternative will most likely be feasible risk-wise. To show how NExpert object is used to implement this part, the queries for risk categories and probabilities proceed as follows for the steel sheet Piles GWS alternative.

what is the Likelihood of Catastrophic Damage of ssp ? If catastrophic damage likelihood is less than a specified threshold, the solution carries on. If not, the plan fails, and SSP will be infeasible. Assume it is 0 .

$>$ o

What is the Likelihood of

Element_Damage_Given_worse_Than_Expected_Conditions ?

$>0.22$

Figure 6.2 maps the probability input into the risk assessment decision tree. 


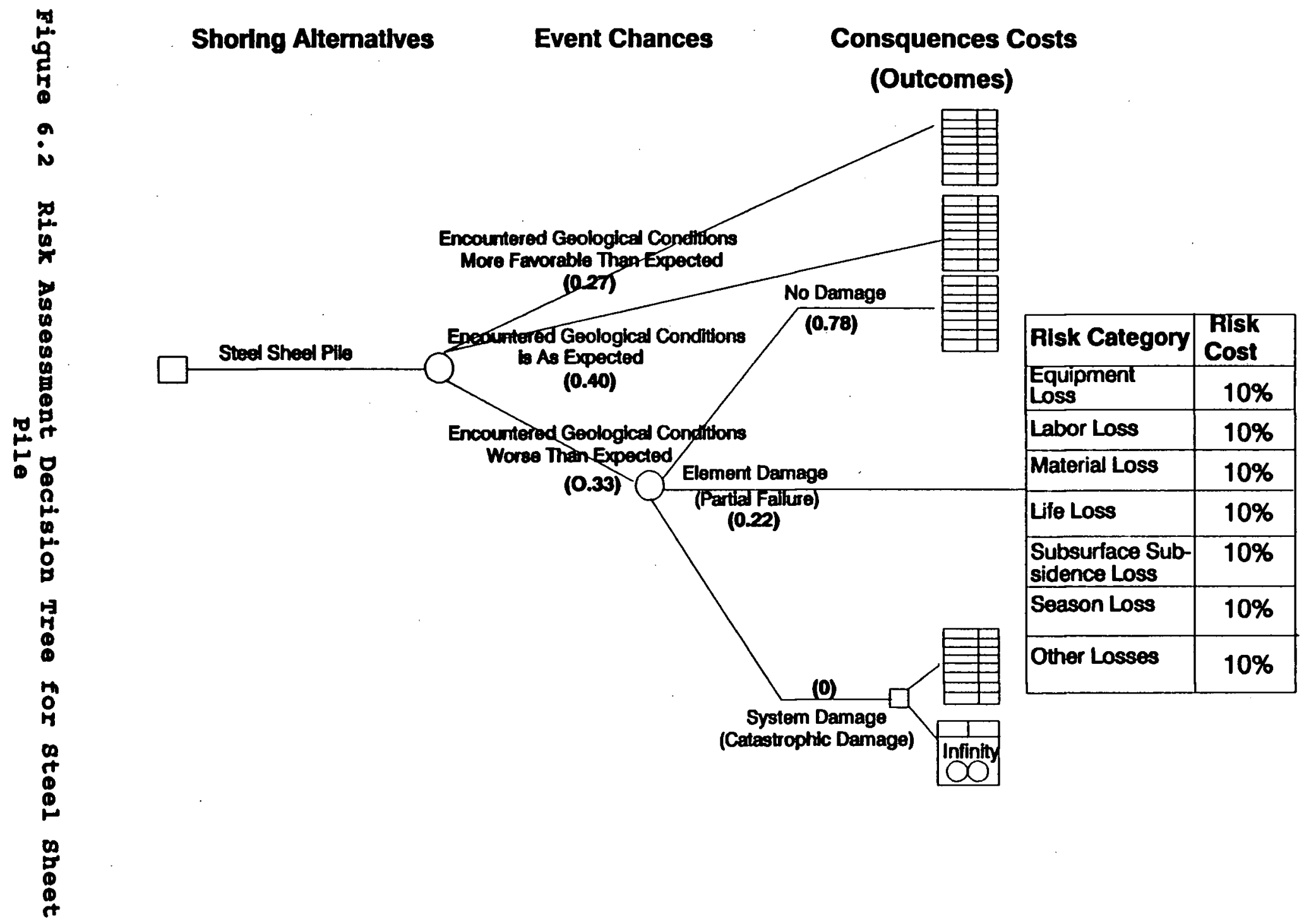

0
0
0
0
0
0
0
0
0
0
0
0
0
0
0
0
0
0
0
0
0
0 
Next, a breakdown of the costs, by resource type and site conditions, is specified by the user, in terms of a fraction of the initial cost for each alternative previously input by the user. Everything is priced against the single input number of expected cost as estimated by the detailed feasibility component.

starting with the worse_Than_Expected conditions scenario, the following risk categories costs are relevant to Element_Damage_Given_worse_conditions scenario. The risk categories values are shown below:

- What is the Equipment Loss

- What is the Labor Loss

- What is the Material Loss

- What is the Life Loss

- What is the subsurface subsidence Loss

- What is the season Loss

- What is the other Losses

$$
\begin{aligned}
& =0.10 \\
& =0.10 \\
& =0.10 \\
& =0.10 \\
& =0.10 \\
& =0.10 \\
& =0.10
\end{aligned}
$$

A calculation takes place to find the likelihood of no element damage given worse than expected condition scenario, Likelihood of No_Element_Damage_Given_worse_Conditions =1-(Likelihood of Element_Damage_Given_worse_Conditions) -(Likelihood of Catastrophic_Damage_Given_worse_Conditions) The likelihood for the catastrophic system failure is assumed to be 0 , therefore, the no damage likelihood is: $=1-0.22-0=0.78$ 
Risk categories relevant to this component are listed below:

- What is the Equipment Loss

- What is the Labor Loss

- What is the Material Loss

- What is the Life Loss

- What is the subsurface subsidence Loss

- what is the season Loss

- What is the other Losses

$$
\begin{aligned}
& =0.00 \\
& =0.00 \\
& =0.00 \\
& =0.00 \\
& =0.00 \\
& =0.00 \\
& =0.00
\end{aligned}
$$

what is the Likelihood of "As_Expected" conditions ? $>40 \%$

Similarly, other risk categories, relevant to the As_Expected scenario of encountered soil conditions, will be considered:

- What is the Equipment Loss

- What is the Labor Loss

- What is the Material Loss

- What is the Life Loss

- What is the subsurface subsidence Loss

- What is the Season Loss

- What is the other Losses

$$
\begin{aligned}
& =0.00 \\
& =0.00 \\
& =0.00 \\
& =0.00 \\
& =0.00 \\
& =0.00 \\
& =0.00
\end{aligned}
$$

The likelihood of the More_Favorable_Than_Expected subsurface conditions is:

$=(1-$ Likelihood of Worse_Than_Expected Conditions Likelihood of As_Expected Conditions)

$$
=(1-0.33-0.40)=0.27
$$

Similarly, other relevant risk categories to the

More_Favorable_Than_Expected scenario of encountered soil conditions will be treated; noting that a negative risk fraction indicates savings. 
- What is the Equipment Loss

- What is the Labor Loss

- What is the Material Loss

- What is the Life Loss

- What is the subsurface subsidence Loss

- What is the season Loss

- What is the other Losses

$=-0.01$

$=-0.01$

$=-0.01$

$=-0.01$

$=-0.01$

$=-0.01$

$=-0.01$

Costs may now be calculated as follows:

Total Cost for the SSP Alternative =

Initial cost for SSP + SSP Risk

Since risk is expressed as percentage of total cost, then

Total cost = Initial cost ( $1+$ SSP_Risk fraction))

Total cost for the SSP Alternative $=\$ 3.00 \mathrm{M} *(1+0.03)$

$$
\begin{aligned}
& =\$ 3.09 \mathrm{M} \\
\text { SSP Risk } & =\$ 90,000
\end{aligned}
$$

Similarly, the process will be repeated for the GWS of the Soldier Piles and Lagging alternative. Table 6.1 summarizes all input data for both GWSS alternatives.

SPL costs are calculated as follows:

Total cost for the SPL Alternative $=$

Initial cost for SPL + SPL_Risk

Total Cost for the SPL Alternative $=2.80 *(1+0.14)$ $\begin{array}{lll} & =\$ 3.19 \mathrm{M} \\ \text { SPL Risk } & =\$ 390,000\end{array}$

Based upon total costs including risks, the steel sheet pile alternative is preferred to the soldier pile and lagging alternative. At the end of the session, a text file will be displayed, indicating both alternatives are feasible and ranking the alternatives in a descending order of total 
costs. Thus, the steel sheet pile alternative will be ranked first, followed by soldier piles and lagging.

Figure 6.3 shows a flow chart of the risk assessment algorithm as implemented.

\subsubsection{Risk Routine}

The risk component is implemented in "Risk.c" routine as an external program. The executable file "risk.exe" is fired by using a rule in the CMSA prototype. The routine basically performs the risk assessment computation as described in section 4.4. The "Risk.c" input data file includes the risk cost categories and probabilities for each alternative. The routine returns the results to an output file where CMSA then retrieves the output variable for further computation. For example, table 6.2 shows the input file "SSP_Risk.nxp" for the SSP alternative. The output file "Riskl.nxp" contains the variable "SSP_Risk.Fraction= $0.0319 "$ "wich is the sum of the risk assessment utility as a fraction of the initial cost. Thus, the total cost for the SSP alternative including risk is equal to $(1+$ SSP_Risk.Fraction) times the initial cost as estimated by CMSA. 
- Worse_Than_Expected

- Catastrophic_Damage

- Element_Damage

- No_Damage

- As_Expected

- Favorable_Than_Expected

Likelihood of Scenarios Conditions SSP Alternative

0.33

0.00

0.22

0.78

0.40

0.27

0.00

0.00

Element_Damage

Equipment Loss $\quad 0.10$

0.10

0.20

Labor Loss

0.10

0.20

Material Loss

0.10

0.20

Life Loss

Subsurface Subsidence Loss

0.10

0.20

0.10

0.20

Season Loss

Other_Losses

0.10

0.20

0.20

No_Element Damage

Equipment Loss

0.00

0.00

Labor Loss

Material Loss

Life Loss

Subsurface Subsidence Loss

Season Loss

Other_Losses

0.00

0.00

0.00

0.00

0.00

0.00

0.00

0.00

0.00

0.00

0.00

0.00

Risk Categories for As_Expected

$\begin{array}{lll}\text { Equipment Loss } & 0.00 & -0.01 \\ \text { Labor Loss } & 0.00 & -0.01 \\ \text { Material Loss } & 0.00 & -0.01 \\ \text { Life Loss } & 0.00 & -0.01 \\ \text { Subsurface Subsidence Loss } & 0.00 & -0.01 \\ \text { Season Loss } & 0.00 & -0.01 \\ \text { Other_Losses } & 0.00 & -0.01\end{array}$

Risk Categories for More_Favorable_Than_Expected

$\begin{array}{lll}\text { Equipment Loss } & -0.01 & -0.02 \\ \text { Labor Loss } & -0.01 & -0.02 \\ \text { Material Loss } & -0.01 & -0.02 \\ \text { Life Loss } & -0.01 & -0.02 \\ \text { Subsurface Subsidence Loss } & -0.01 & -0.02 \\ \text { Season Loss } & -0.01 & -0.02 \\ \text { Other_Losses } & -0.01 & -0.02\end{array}$

Table 6.1 Risk Assessment Data Input Summary 


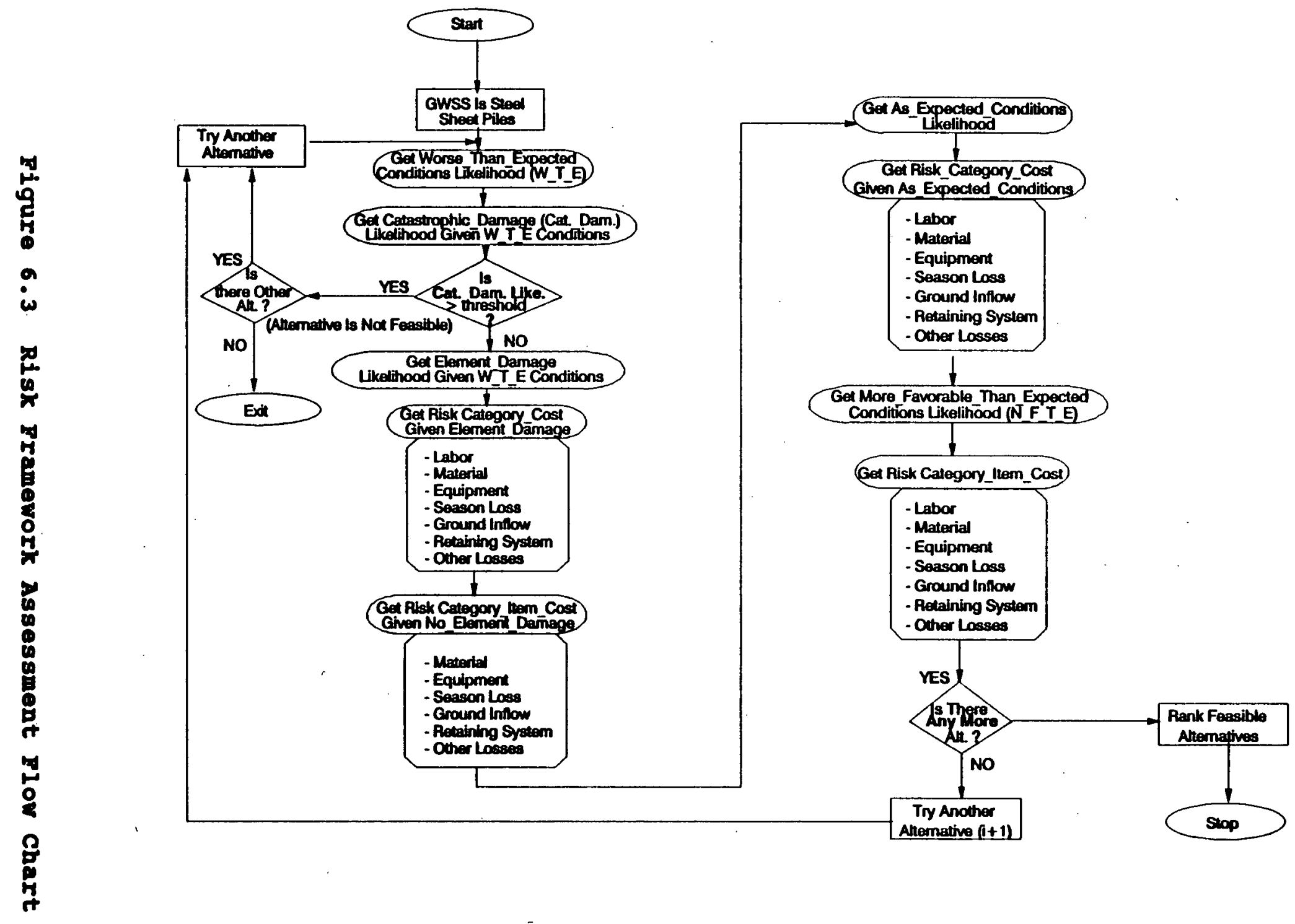

0
0
0
0
0
0
0
0
0
0
0
0
0
0
0
0
0
0
0
0
0 
|Favorable_Than_Expected.Probability $\backslash=" 0.27 "$

$\langle$ Equipment_Loss_for_FTE $\backslash="-0.010 "$

Labor Loss for $\overline{\text { FTE }} \backslash="-0.010 "$

$\mid$ Material_Loss_for FTE $\mid="-0.010 "$

$\backslash$ Subsurface Subs_Loss for FTE $\backslash="-0.010 "$

$\backslash$ Season_Loss_for_FTE $\rangle^{-}="-0.010^{\prime \prime}$

$\backslash$ Life_Loss_for_FTE $\backslash "-0.010 "$

|Other_Losses_for_FTE $\backslash="-0.010^{\prime \prime}$

\Favorable_Thān_Expected.Finish \=" "

$\backslash$ As_Expected.Probability $\backslash=" 0.40 "$

$\backslash$ Equipment_Loss_for_AE $\backslash=" 0.00 "$

$\backslash$ Labor_Loss_for_A $\bar{A} \backslash \overline{ } \backslash=" 0.00 "$

Material_Loss_for_AE $\mid=$ "0.00"

$\mid$ Subsurface_Subs_Loss_for_AE $\backslash=" 0.00 "$

$\langle$ Season_Loss_for_AE $\rangle=" 0.00 "$

$\backslash$ Life_Loss_for_A $\overline{\mathrm{E}} \backslash " 0.00^{\prime \prime}$

OOther_Losses_for_AE $\backslash=" 0.00 "$

$\backslash$ As_Expected.F.

\Worse_Than_Expected.Probability $\backslash=" 0.33 "$

No_Damage_Given_W T E.Probability $\backslash=" 0.78 "$

$\backslash$ Equipment_Loss_for_ND $\overline{\mathrm{N}} \backslash=" 0.00 "$

Labor_Loss_for_ $\overline{\mathrm{ND}} \backslash=" 0.00 "$

$\backslash$ Material_Loss_for_ND $\backslash=" 0.00 "$

$\backslash$ Subsurface_Subs_Loss_for_ND $\backslash=" 0.00 "$

$\backslash$ Season_Loss_for_ND $\backslash=" 0.00 "$

$\backslash$ Life_Loss_for_ $\mathrm{ND} \backslash " 0.00 "$

$\backslash$ Other_Losses_for_ND $\backslash=" 0.00 "$

$\backslash$ No_Dämage_Given_W_T_E.Finish $\backslash="$ "

$\backslash$ Element_Damage_Given_W_T_E.Probability $\backslash=" 0.22 "$

(Equipment_Loss_for_ED $\bar{\top}=\overline{ } \overline{0} 0 . \overline{1} 0 "$

$\backslash$ Labor_Loss for $\overline{\mathrm{E} D} \backslash \overline{-}=0.10^{\prime \prime}$

$\backslash$ Material_Loss_for_ED $\backslash=" 0.10 "$

$\backslash$ Subsurface_Subs_Loss_for_ED $\backslash=" 0.10 "$

$\langle$ Season_Loss_for_ED $\backslash=" 0 . \overline{1} 0 "$

$\backslash$ Life Loss for E $\overline{\mathrm{D}} \backslash$ "0.10"

|Other_Losses_for_ED $\backslash=" 0.10 "$

\Element Damage Given W T E.Finish $\backslash=$ " "

$\backslash$ System_Damage_Given $\overline{\mathrm{W}} \overline{\mathrm{T}}$ E. $\bar{E}$.Probability $\backslash=" 0.00 "$

$\backslash$ Equipment_Loss_for_S $\bar{D} \backslash=" \overline{0.00 "}$

$\backslash$ Labor_Loss_for_S $\mathrm{S}\rangle^{-}=" 0.00$ "

$\backslash$ Material_Loss_for_SD $\backslash=" 0.00 "$

$\backslash$ Subsurface_Subs_Loss_for_SD $\backslash=" 0.00 "$

$\backslash$ Season_Loss for_SD $\backslash \overline{=}=\overline{0} 00 "$

$\backslash$ Life Loss for $\mathrm{S} \overline{\mathrm{D}} \backslash " 0.00 "$

$\backslash$ Other_Losses_for_SD $\backslash=" 0.00^{n}$

$\backslash$ System_Damäge_G̈iven_W_T_E.Finish $\backslash="$ "

\Worse_Than_Expected.Finish $\backslash="$ "

Table 6.2 "SSP_Risk.nxp" Input File for ssp Alternative 


\section{Conclusions and Recommendations for Further Research}

\subsection{Summary}

This thesis reveals that the problem of construction methods selection is complex and ill-structured. A tool is needed to categorize, rank, prune, and synthesize methods alternatives. A step in this direction was provided through a KBES framework approach, which further led to the concept of a construction methods selection shell. A prototype system called CMSA was designed and implemented.

This system interacts with the user to suggest, design, predict, and analyze the assembled method. Presently, CMSA may be considered as a developmental stage prototype, i.e., an expert system which has passed the conceptual stage, and where a first prototype has been built and run but not completely validated. Several method attributes, goals, and constraint constructs (or primitives) have been implemented. The structure adopted will allow other researchers to add other methods, alter some rules, and adapt CMSA to different construction problem domains as desired. 


\subsection{Contribution of The Thesis}

The main contributions of this thesis are:

1. Structuring of the problem of method selections by setting out a generalized definition of the construction method selection problem (Chapter 4) which can accommodate a diverse range of methods selection problems.

2. The devising of an expert system conceptual model involving declarative and procedural knowledge which reflects a practical approach to generating and then reducing a feasible set of alternatives. A two tiered process of preliminary and detailed feasibility is proposed to screen, prune, and synthesize a construction method.

3. The implementing of a small prototype, called CMSA, to demonstrate the feasibility of the detailed feasibility part architecture proposed in the conceptual model. Most of the features identified in the detailed feasibility part of the conceptual model were implemented in CMSA. 


\subsection{Further Research}

Listed below are several avenues to further pursue in order to fully explore the potential for knowledge based systems to make a substantive contribution to productivity improvement through development of a construction methods selection tool.

1. Attention should be focused on exploring a range of technology rich example problems such as Cut-and-Cover tunnelling, structural cycle design for high rise construction, bridge construction and so forth in order to further generalize the conceptual model proposed.

2. The preliminary feasibility phase component of the model should be implemented and further elaborated on; undertaking further knowledge acquisition, implementing the proposed data base for methods, enlarge the regulation knowledge component, and so forth.

3. At the detailed feasibility level, the use of optimization techniques to affect tradeoffs between fixed and variable costs (e.g. spacing of retaining system members) should be explored. 
4. Experiment implementing CMSA using a purely object oriented Programming (OOP) approach. For instance, this can be applied for technical feasibility of a method as a generalized and parental object. such objects can begat other objects such as method designs, strategies, model processes, and construction processes. This involves object design, different inference mechanisms (message exchanges), and should be compared to the previous implementation in terms of possible code size reduction, and ease of understanding.

5. Addressing validation issues for CMSA through more input from practitioners. In some instances, where knowledge was represented as high primitives, it is preferable that such knowledge be available in the system in a more objective format. For instance, in the risk assessment of a shoring alternative, it is assumed that the user estimates the value of GWSS wall failure probabilities based on project context and water table conditions.

6. More documentation of methods and the procedural and experiential knowledge that accommodates them, is needed. For instance, there are incomplete knowledge 
bases such as the vibratory hammer $K B$ which need further procedures to estimate productivity. Most design KBs (SSP, SPL, Struts, and wales) employed one or two design methods. other design methods, which may be more suitable under a specific project context, could be included. 


\section{Bibliography}

[1] Adeli, H., and Paek, Y.J. (1986)., "Computer-Aided Design of Structures Using Lisp.", Computers \& Structures, Vol.22, No.6, pp.939-956.

[2] AISC (Nov 1978)., Specification for the Design, Fabrication and Erection of Structural Steel Buildings., Chicago: American Institute of Steel Construction Inc.

[3] Ashley, D.B. (1980).,"Simulation of Repetitive Unit Construction.", Journal of Construction Engineering and Management, Vol.106, No.CO2, pp.185-195.

[4] Ashley, D.B. et al. (1983)., "Critical Decision Making During Construction.", Journal of Construction Engineering and Management, Vol.109, No.2, pp.146162.

[5] Ashley, D.B. et. al. (1979)., "Advantage and Limitations of Adaptable Tunnel Design and Construction Methods.", Proceedings of Rapid Excavation and Tunnelling Conference, Atlanta, Georgia, U.S., pp.989-1011.

[6] Ayyub, B., and Halder, A. (Dec 1985). "Decisions in Construction Operations.", Journal of Construction Engineering and Management, Vol.111, No.4, pp.343-357.

[7] Barber, G. H. (1987)., "Fitting the Hammer to the Pile and the Soil", Pile Buck, Pile Buck Inc., First June Issue, P.O.Box 1056, Jupiter, FL 33468-1056, pp. 26-34.

[8] Bernold, L.E. (1987)., "Goal Oriented Expert-Simulation For Building Construction.", Transaction of the Building Cost Research Conference on Building Cost Models and Computers, pp.409-418.

[9] Bickel, J.O., and Kuesel, T.R. (1982)., Tunnel Engineering Handbook, New York: Van Nostrand Reinhold Co.

[10] Blanning, R.W. (1984)., "Management Applications of Expert Systems.", Information and Management, No.7, pp.311-316.

[11] Bobrow, D.G., and Stefik, M. (1983)., The Loops Manual, Xerox Corporation.

[12] Bonissone, P.P., and Tong, R.M. (1985). "Editorial: Reasoning with Uncertainty in Expert Systems.", International Journal of Man-Machine Studies, No.22, pp.241-250. 
[13] Bowles, J.E. (1977)., Foundation Analysis and Design, New York: McGrawHill Inc.

[14] Bruce, D. A., and Jewell, R. A. (1987)., "Soil Nailing: Applications and Practice, Part (2).", Ground Engineering, Vol.20, No.1, pp. 10-15.

[15] Buchanan, B.G, and Shortliffe, S.H.(1984)., Rule Based Expert Systems, Reading, Mass.: Addison-Wesley Pub. Co.

[16] Canadian Foundation Engineering Manual (1978)., Canadian Geotechnical Society, Montreal, Quebec, Canada.

[17] Caterpillar Performance Handbook (1982)., Caterpillar Tractor Co., Peoria, Illinois, U.S.A.

[18] Charniak, E., and McDermott, D. (1985)., Introduction to Artificial Intelligence., Reading, Mass.: Addison-Wisely Pub. Co.

[19] Chin, M. W. (1987)., "Some Potential Applications of Expert Systems in Construction Engineering and Management in the West Indies", Managing Construction Worldwide, Vol.1, London, U.K., pp.591-597.

[20] Clancey, W. J. (Dec 1985)., "Heuristic Classification", Artificial Intelligence, Vol. 27, pp.289-350.

[21] Clocksin, W.F., and Mellish, C. S. (1981)., Programming in Prolog, Berlin: Springer-Verlag.

[22] CSA Standard 085-1976 (1976)., "Code for the Engineering Design of Wood.", Canadian Standard Association, Rexdale, Ontario, Canada.

[23] Duda, R.O., Gasching, J.G., and Hart, P.E. (1979)., Model Design in the PROSPECTOR Consultant System for Mineral Exploration. Expert Systems in the Micro Electronic Age, Michie, D., ed. (1979)., Edinburgh: Edinburgh University Press.

[24] Duda, R.O., Hart, P.E., Nilsson, N.J., and Sutherland, G. (1978)., "Semantic Network Representation in Rule Based Inference systems.", Pattern Directed Inference Systems, Waterman,D., and Hays-Roth, F., (Eds.) New York: Academic Press.

[25] Gaarslev, A. (1977)., "Optimizing Interrelated Decisions in Construction Plannings.", Relations in Project Management, Ninth Project Management Institute Conference, Drexel-Hill, Pennsylvania, pp.344-352.

[26] Gashing, J. et al. (Aug 1981)., "Developments of a Knowledge Based System for Water Resources Problems.", SRI Project 1619, SRI International, Stanford, California, U.S.A. 
[27] Gates, M., and Scarpa, A., (1980)., "Criteria for Selection of Construction Equipment.", Journal of Construction Engineering and Management, Vol.106, No.2, pp.207-219.

[28] Gates, M., and Scarapa, A. (1984)., "Optimum Penetration of Friction Piles.", Journal of Construction Engineering and Management, Vol. 110, No.4, pp.491510.

[29] Goldberg, A. (1981)., "Introducing the Smalltalk-80 System, BYTE, Vol.6, No.8, pp.36-48.

[30] Gray, C.A., and Little J. (1985)., "A Systematic Approach to The Selection of An Appropriate Crane for a Construction Site.", Construction Management and Economics, No.3, pp.121-144.

[31] Gray, C.A. (1986)., "Intelligent Construction Time and Cost Analysis.", Construction Management and Economics, No.4, pp.135-150.

[32] Gruber, T., and Cohen, P. (1987)., "Principles of Design for Knowledge Acquisition.", IEEE Conference on Artificial Intelligence Applications, pp.9-15.

[33] Halpin, D.W., and Bernold, L.E. (1986)., "Advance Microcomputer Simulation for Construction Managers.", Proceedings of the 4th Conference on Computing in Civil Engineering, New York, N.Y., pp. 762-772.

[34] Halpin, D.W., and Woodhead, R.W. (1976)., Design of Construction and Process Operations, New York: John Wiley and Sons.

[35] Harris, F., and Alkass, S. (1988)., "Expert System for Earthmoving Equipment Selection in Road Construction.", Journal of Construction Engineering and Management, Vol.114, No.3, pp.344-363.

[36] Harris, F.C., and Wijesundera, A (1985)., "The Integration of an ES in the Construction Planning Process.", Proceedings of 2nd International Conference of Civil and Structural Engineering Computing, Vol.2, pp.399-405.

[37] Hendrickson, C.T. et al. (1988)., "Expert Systems for Construction Planning.", Journal of Computing in Civil Engineering, Vol.1, No.4, pp.253-269.

[38] Hendrickson, C.T. et al. (1987)., "Hierarchical Rule-Based Activity Duration Estimation", Journal of Construction Engineering and Management, Vol.113, No.2, pp.288-301.

[39] Howard, R.A. (1983)., "The Evolution of Decision Analysis.", Readings on The Principle and Applications of Decision Analysis, Vol.1: General Collections, Howard, R. A. and Matheson, J.E. (Eds.), Strategic Decisions Group, pp.516.

[40] Hunt, H.W. (1979)., Design and Installation of Driven Pile Foundation, Associated Pile and Fitting Corp., Clifton, New Jersey, U.S.A. 
[41] Hurd, M.K. (1961)., "Formwork For Concrete", ACI Special Publication No. 4, Detroit, Illinois.

[42] Jackson, P. (1986),, Introduction to Expert Systems., U.K., Wokingham: Addison-Wesley Pub. Co.

[43] Jones, J.P., and Newcomb, F.M. (1963). "Introduction to Pile Driving", TR No.23, Construction Institute of Stanford University, Stanford, CA., U.S.A.

[44] Kim, Y.W. (1984)., Decision Support Framework in Adaptable Tunnelling, Ph.D Thesis, Department of Civil Engineering, Cambridge, MIT, U.S.A.

[45] Kunz, J.C. et al. (1986)., "Contingent Analysis for Project Management Using Multiple Worlds.", Proceedings of First International Conference on Application of Artificial Intelligence in Engineering Problems, London, U.K., pp.707-718.

[46] Law, G.K. (1987)., Decision Support System for Construction Cycle Design, Master Thesis, Department of Civil Engineering, University of British Columbia, Vancouver, Canada.

[47] Levitt, R.E., and Ashley, D.B. (1987)., "Expert Systems in Construction: Work in Progress.", Journal of Computing in Civil Engineering, Vol.1, No.4, pp.303311.

[48] Levitt, R.E., Kartam, N.A., and Kunz, J.C. (1988)., "Artificial Intelligence Techniques for Generating Construction Projects Plans.", Journal of Construction Engineering and Management, Vol.114, No.3, pp.329-343.

[49] Logcher, R.D., and Leston, N.B. (1985)., "An Expert System Framework for Analyzing Construction Project Risks.", Center for Construction Research and Education, Department of Civil Engineering, MIT, U.S.A.

[50] Lutz, J.D., Chang, L., and Napier, T. (1990)., "Evaluation of New Building Technology", Journal of Construction Engineering and Management, Vol.116, No.2, pp.281-299.

[51] McDermott, J. (Fall 1984)., "R1 revisited: Four years in the trenches.", $A I$ Magazine, pp.21-32.

[52] Means Heavy Construction Cost Data (1987)., R.S. Means Company Inc., 1st Annual Edition, Kingston, Massachusetts, U.S.A.

[53] Megaw, T.M, and Bartlett, J.V. eds. (1981)., Tunnels: Planning, Design, Construction., Vol.1 and Vol.2, U.K., Chicester: Ellis Horwood Limited.

[54] Mellichamp J.M., and Wahab, A.F. (1987)., "An Expert System for FMS Design.", Simulation, Vol.48, No.5, pp.201-208.

[55] Meritt, R.S. ed. (1976)., Standard Handbook for Civil Engineering, New York: McGraw-Hill Book Company. 
[56] Mikroudis, G., and Wilson, J.L. (1986)., "Selecting a Tool for Building a Knowledge-Based System.", ATLSS Report No. 86-01, Lehigh University, Lehigh, U.K.

[57] Miller, H.D., and Grant, D. (1987). "Tunnel Simulation Computer Model", Science Council of British Columbia, Vancouver, B.C., Canada.

[58] Minsky, M., (1968)., Semantic Information Processing. Cambridge, Mass.: MIT Press.

[59] Minsky, M., (1975)., "A framework for Representing Knowledge.", Artificial Intelligence, Winston, P.H., ed., 1986, Mass.: Addison-Wesley Pub. Co.

[60] Mohan, S. (1990)., "Expert Systems Applications in Construction Management and Engineering", Journal of Construction Engineering and Management, Vol.16, No.1, pp.87-99.

[61] Murray, K.J., and Sheppard, S.V. (1988)., "Knowledge Based Simulation Model Specification.", Simulation, Vol.50, No.3, pp.112-119.

[62] Neuron Data Inc. (1988)., NExpert Object Fundamentals: PC Version 1.1, Palo Alto, California, U.S.A.

[63] O'Keefe, R.M. (1986)., "Simulation and Expert Systems -- A Taxonomy and some Examples.", Simulation, Vol. 46, No.1, pp.10-16.

[64] Palmer, D.J, and Tomlinsan, M. (1981)., "Are piles necessary and what determine choice?", U.S. Department of Transportation Reports FHWA-RD73-40 \& 41, pp.111-120.

[65] Paulson, B.C. et al. (1981)., "Simulation and Analysis of Construction Operations.", Journal of Construction Engineering and Management, Vol.109, No.2, pp. 89-104.

[66] Paulson, B.C. et al. (1987)., "Simulation and Analysis of Construction Operations by Microcomputers." Journal of Construction Engineering and Management, Vol.113, No.2, pp.302-314.

[67] Peck, R.B. (1984)., "State of The Art : Soft Ground Tunneling.", Proceedings of two sessions at Geotek'84, Tunnelling in Soil and Rock, Atlanta, Georgia, pp.1-11.

[68] Peurifoy, R.L. (1970)., Construction Planning, Equipment, and Methods, 2nd Edition, New York: McGraw-Hill Book Co.

[69] Ratay, R.T. (1984)., Temporary Structures in Construction, New York: McGraw-Hill Book Co. 
[70] Reddy, Y.V., and Fox M. (1982)., "Knowledge Representation in Organization Modeling and Simulation: A Detailed Example.", Proceedings of 13th Annual Pittsburgh Conference, April 1982, pp.685-692.

[71] Riggs, L.S. (1980)., "Simulation of Construction Operations"., Journal of Technical Issues, Vol.106, No.1, pp.145-163.

[72] Ringwald, R.C. (1987)., "Bunching Theory Applied to Minimize Cost", Journal of Construction Engineering and Management, Vol.113, No.2, pp.321326.

[73] Schroeder, W.L. (1980)., Soils in Construction, 2nd ed., New York: John Wiley \& Sons.

[74] Selinger, S. (1980)., "Construction Planning for Linear Projects." Journal of Construction Engineering and Management, Vol. 106, No.2, June 1980, pp.195205.

[75] Shafer, G. W. (1976)., A Mathematical Theory of Evidence. Princeton, New Jersey: Princeton University Press.

[76] Shannon, R.E. (1986). "Intelligent Simulation Environments.", SCS Simulation Series,Vol.17, No.1, pp.150-156.

[77] Shannon, R.E. (1987)., "Models and Artificial Intelligence.", Proceedings of the 1987 Winter Simulation Conference, pp.16-23.

[78] Shen, C.K. et al. (1981a)., "Field Measurement of an Earth Support System.", $A S C E$ GT12, pp.1625-1642.

[79] Shen, C.K. et al. (1981b)., "Ground Movement Analysis of Earth Support Systems.", $A S C E$ GT12, pp.1609-1624.

[80] Tatum, C.B. (1987)., "Improving Constructibility During Conceptual Planning.", Journal of Construction Engineering and Management, Vol.113, No.2, pp.191-207.

[81] Tatum, C.B. (1988)., "Classification System for Construction Technology.", Journal of Construction Engineering and Management, Vol.114, No.3, pp.344363.

[82] Tomlinson, M.J. (1975)., Foundation Design and Construction, 3rd ed., U.K., London: Pitman Pub. Co.

[83] Toolan, F. E., and Fox, D. A. (1977). "Geotechnical Planning of Piled foundations for Offshore Platforms.", Proceedings of Civil Engineering Institute Part-1, May 1977, p.221-244.

[84] Touran, A., and Toshyuki, A. (1987)., "Simulation of Tunnelling Operations.", Journal of Construction Engineering and Management, Vol.113, No. 4, pp.554568. 
[85] Trimble, G. (1987)., "Expert Systems in Construction: Their Potentials and the Methodology of Knowledge Acquisition.", Managing Construction Worldwide, Vol.1, London, U.K., Sept 1987, pp.610-619.

[86] Turban, E., and Watkins, P. (1985)., "Integrations of Expert System and Decision Support System.", DSS-85, pp.52-63.

[87] Touran, A. (1987)., "Integration of Expert Systems with Simulation.", Journal of Construction Engineering and Management, Vol.11, No.3, pp.480-493.

[88] Wager, D.M. (1987)., "Can Expert Systems Help the Construction Industry.", Proceedings of the second International Conference in Civil and Structural Engineering Computing, Vol.2, London, U.K.,pp.387-389.

[89] Warszwaski, A. (1985). "Decision Models and Expert Systems in Construction Management.", Building and Environment, Vol.20, No.4, pp.201210.

[90] Winterkorn, H.F., and Fang, H., Eds. (1975)., Foundation Engineering Handbook, New York: Van Nostrand Reinhold Co. Inc.

[91] Winston, P.H. (ed.), (1986)., Artificial Intelligence, 2nd edition, Reading, Mass.: Addison-Wesley Pub. Co.

[92] WEAP Program (1983)., Goble and Associates Inc., U.S. Department of Transportation., Washington D.C., U.S.A

[93] Zadeh, L. A. (1975)., "Fuzzy Logic and Approximate Reasoning", Syntheses, Vol.30, pp.407-428. 


\section{Appendix A}

\section{Earth Pressure and Moments Computations}

\section{A.1 Introduction}

Soil is an extremely heterogeneous material that has been classified under different classification systems into many types. For purposes of this thesis, soil types are restricted to a subset of sand (loose sand and dense sand), and clay (soft clay and stiff clay). Furthermore, soil profile layering is restricted to one or two layers for these soil types. Thus, 14 soil layering scenarios are possible, e.g., loose sand for one layer, loose sand on stiff clay for two layers, etc.

Two major soil interactions relevant to the design of ground wall support structure are lateral pressure and moments.

\section{A.2 Lateral Pressure Calculations}

Lateral pressure is a function of soil type, retaining system specification (vertical and horizontal spacing, its type, e.g., anchorage vs. struts), and rate of construction. Conventional earth lateral pressure and moment computation methods (Peck 1969, Terzaghi and Peck 1967, and Winterkorn and Fang 1975) are available as well as sophisticated Finite Element Methods. The former are adopted for the prototype CMSA implementation as we wish to explore alternative solutions, rather then refine the design of specific GWSSs. Formulas, set out in several references (Ratay 1984, and Winterkorn and Fang 1975) were adopted for estimating earth pressure and moments.

The following nomenclature is adopted.

\section{Nomenclature}

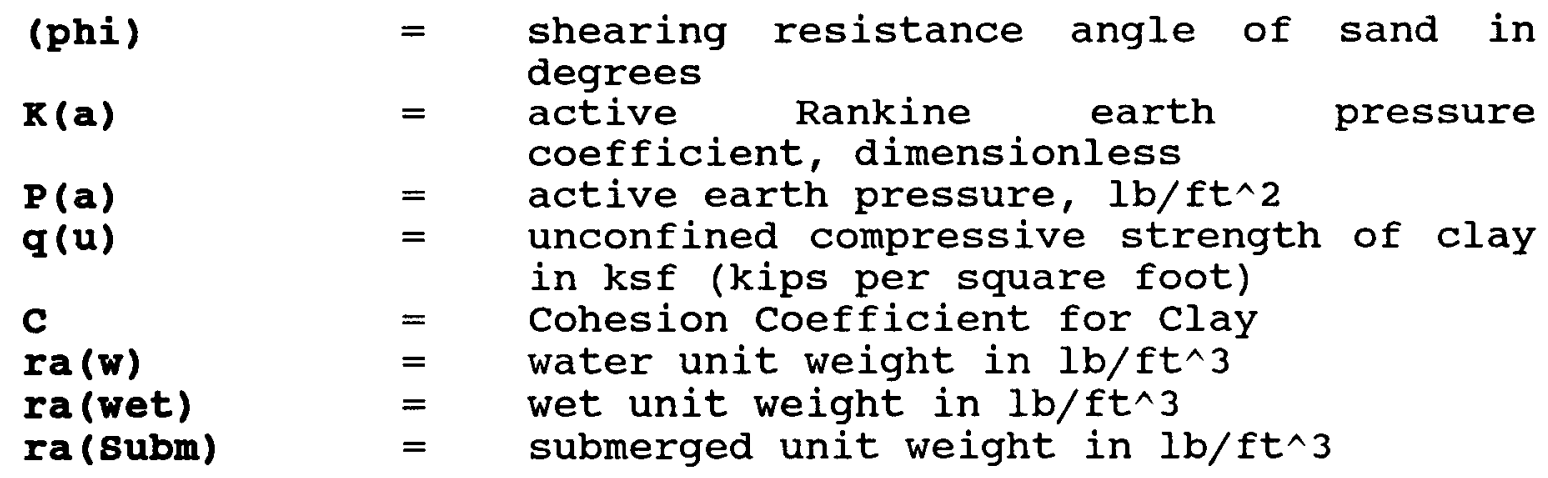




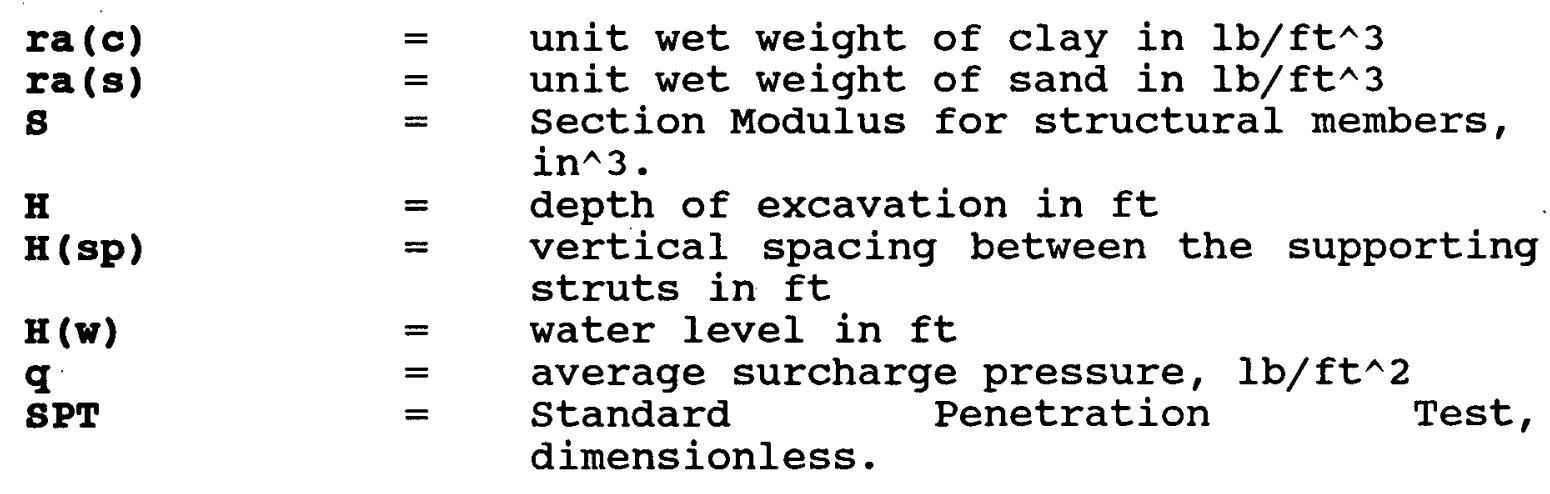

Soil properties adopted for the CMSA prototype are shown in table A. 1 .

\begin{tabular}{|llllll|}
\hline Soil_Type & ra & phi & q(u) & SPT \\
(wet) & $\begin{array}{l}\text { ra } \\
\text { (subm) }\end{array}$ & 58 & 0.70 & & \\
1. Loose_Sand & 100 & 70 & 2.6 & & $30-10$ \\
2. Dense_Sand & 129 & & & 30 & \\
& & 60 & & 40 & $8-15$ \\
3. Soft_Clay & 110 & 70 & & & \\
4. Stiff_Clay & 130 & & & & \\
\hline
\end{tabular}

Table A.1 soil Types properties Employed in CMSA

Next, calculations for pressures and moments envelopes will be derived for one layer and two layer scenarios. 


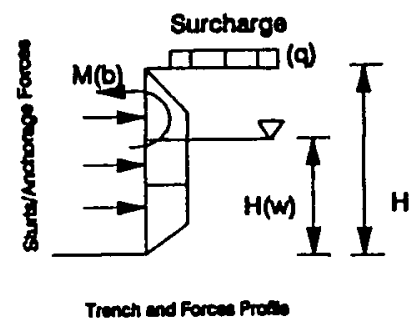

(e)

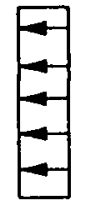

801

(b)

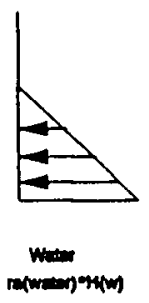

(c)

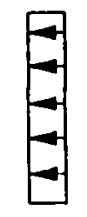

Sureheres
K(a)

(d)

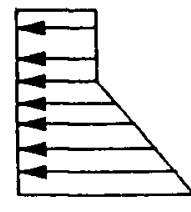

Toted Preceure

(e)

Presaure Dlagrams

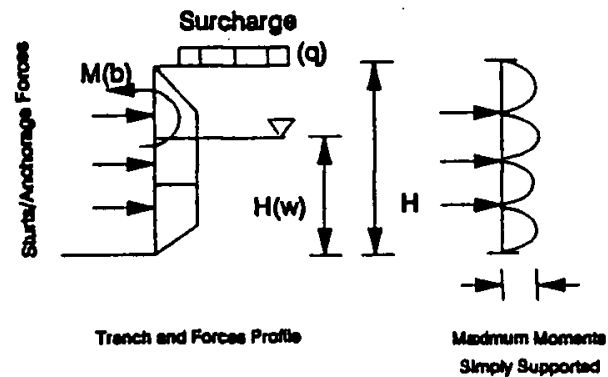

(II)

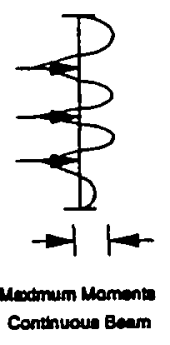

(n)

Figure A.1 Pressure and Moments Envelopes (from winterkorn and Fang 1975)

Figure A.1 (a) shows a sketch of a retaining system along with the system reactions (struts/anchors) and pressure envelopes. The pressure is decomposed into soil pressure (b), water (c), and surcharge (d), and their sum is shown in figure (e). Figures A.I (f) and (g) represent moment diagrams that correspond to total soil profile pressure for simply supported beam (hinged) and continuous (fixed at each end).

For the CMSA prototype, the surcharge pressure was not considered. Next, lateral pressure and moment relation for one and two layer soil models are presented.

\section{A.2.1 One soil Layer}

\section{Case (a): When soil is Loose sand/Dense sand}

Using the Terzaghi-Peck approach (1967), the unit pressure for sand is given by (Winterkorn and Fang 1975):

$\mathrm{P}$ (sand) $=0 . * 65 * \mathrm{~K}(\mathrm{a}) * \mathrm{ra}(\mathrm{sand}) * \mathrm{H}$ 
where

ra(Sand) = dry unit weight for the sand under water table, and wet unit weight above water table

$\mathrm{R}(\mathrm{a})=(\tan (45-(\mathrm{phi}))) \wedge 2$

For temporary structures design, only active Rankine pressure forces have been considered, i.e., passive Rankine pressure is ignored. Rankine Pressure coefficients are computed using coulomb pressure envelopes which do not account for wall soil angle friction.

Maximum unit pressure for water,

$P($ water $)=$ ra(water $) * H(w)$

Therefore, maximum total pressure is

$P($ Total $)=P($ sand $)+P$ (water $)$

Platic water (A.4) bottom of excavation).

Maximum moments (assume uniformly distributed load)

a) for continuous spans,

$M(\max )=P * H(s p) \wedge 2 / 10$

where,

$\mathrm{H}(\max )=$ Maximum vertical spacing between the struts (15 ft is the vertical spacing adopted for CMSA) .

b) for simple spans:

$M(\max )=P * H(s p) \wedge 2 / 8$

(A.6)

For CMSA, equations A. 3 and A. 5 will be adopted with default retaining system spacing of $12 \mathrm{ft}$ horizontally and $15 \mathrm{ft}$ vertically in Ratay (1984), horizontal spacing varies from 8 ft to $12 \mathrm{ft}$ ).

Case (b): when soil is soft clay/stiff clay (Terzaghi-Peck (1967))

For cohesive soils, the water table should be assumed at the soil surface, and total stresses are computed with (phi) = 0 . This applies to short term excavation open for an extended period without standing water. 
For soft clay

$\mathbf{P} \quad \mathbf{R}(\mathrm{a}) *(\mathbf{r a}) * \mathrm{H}$

For stiff clay

$\mathbf{P} \quad=\mathbf{F} \star(\mathbf{r a}) \star \mathbf{H}$

where $F=0.30$

ra(clay) = saturated unit weight for the clay under the water table and wet unit weight above the water table.

\section{A.2.2 Two soil Layers}

For two or more soil layers (see figure A.2), Peck (1943) proposed the following unit pressure for excavations in layered soils (sand and clay), with sand overlaying clay:

$P(m) \quad=R^{\prime}(a) * r a^{\prime} * H+r a(w) *(H-H(w))$

where

$R^{\prime}(a) \quad=1-2 * q^{\prime}(u) /\left(r a^{\prime} * H\right)$

(A. 10)

and $q^{\prime}(u)$ is the average compressive strength for sand and clay, and ra' is the average unit weight for sand and clay.

$q^{\prime}(u)=\underset{H(s)}{(1 / H) *[r a(s)} * H(s) \wedge 2 * K(s) * \tan (p h i)+(H-$

The $s$ subscript refers to sand stratum and $n=$ ratio of field unconfined compressive strength to that determined in the laboratory. Also,

$\mathrm{ra}^{\prime}=(1 / \mathrm{H}) \star[\mathrm{ra}(\mathrm{s}) * \mathrm{H}(\mathrm{s})+\mathrm{ra}(\mathrm{c}) \star \mathrm{H}(\mathrm{c})]$

in which c subscript refers to the clay stratum.

Pressure relationships (and pressure diagrams) for design of retaining elements in mixed soils which have values of $c$ and phi have not been developed (Winterkorn and Fang 1975).

Conventional techniques may be employed for design of bracing elements in shallow excavations. Lambe (Winterkorn and Fang 1975) has reviewed various methods of determining pressures in excavations and soil movements and has compared the results with data from actual excavations. It was shown that movements of the soil outside of the excavation and strut loads cannot be adequately predicted under most field conditions. 


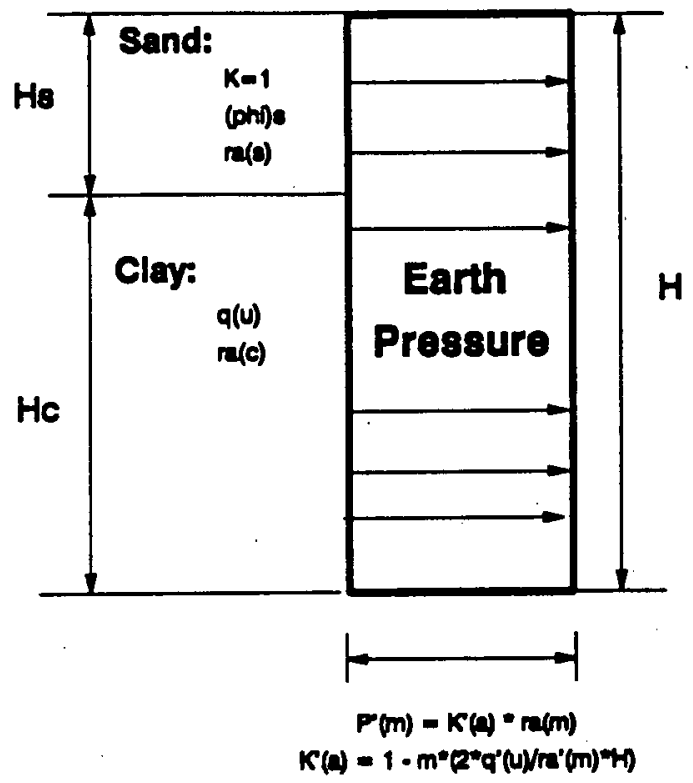

\section{Figure A.2 soil Profile for Two soils scenario [from winterkorn and Fang 1975]}

\section{A.3 Design Principles for Structural Members}

\section{A.3.1 Btructural Member Design (wall Members)}

This section describes design principles for sizing steel sheet piles and soldier piles.

\section{Bteel sheet piles}

a) For continuous spans the maximum moment for 3 or more spans is equal:

$M(\max )=P *\left(H(s p)^{\wedge} 2\right) / 10$

b) For simple spans:

$M(\max )=P *(H(s p) \wedge 2) / 8$

$(8)=M(\max ) /(1.5 * F(b))$

$S$ is the required section modulus, 1.5 is the overstress factor for short term loading, and $F(b)$ is the allowable steel working stress which is assumed to be $25 \mathrm{ksf}$ for A328 steel. 
Example: Size a steel sheet pile for an excavation for $45 \mathrm{ft}$ deep excavation of loose sand. Retaining system spacing is 15 vertically. The water table elevation is $10 \mathrm{ft}$ below the surface.

From equation A.1, maximum Pressure for Sand

$=0.65 * \mathrm{~K}(\mathrm{a}) *$ subm. Unit Weight for loose sand * Excavation depth $(\mathrm{H}+5)$

$=0.65 * 0.33 * 0.058 * 50=0.62 \mathrm{ksf}$

The depth of sheeting below excavation level is assumed to be 5 ft as a default in CMSA to prevent or minimize soil heave or boiling. For more accurate results, a trial and error procedure to find its value can be found in foundation texts (winterkorn and Fang 1975, Ratay 1984) where a 20 to $40 \%$ is typically added to the calculated length as a safety factor in some cases.

From equation A.2, maximum Pressure for water

$=r a($ water $) *(\mathrm{H}-10)$

$=0.062 * 40=2.48 \mathrm{ksf}$

Total Pressure $(P)=0.62+2.48=3.10 \mathrm{ksf}$

Assuming hinges between the lower two struts (simple supported span assumption), the maximum moment, using equation A. 6 is

Moment $=\mathrm{P} * \mathrm{H}(\max ) \wedge 2 / 8$ $=3.10 * 15 \wedge 2 / 8=87.19$ kips.ft

Then from equation A.9, the required section modulus $S$

$$
\begin{aligned}
& =\begin{array}{l}
\text { Moment*12/(working stress * } \\
\text { overstress factor }(1.5) \text { ) }
\end{array} \\
& =\quad 27.9 \mathrm{in} \wedge 3 / \mathrm{ft} \text { of wall }
\end{aligned}
$$

From the ASTM A328 data base lookup, a section modulus of

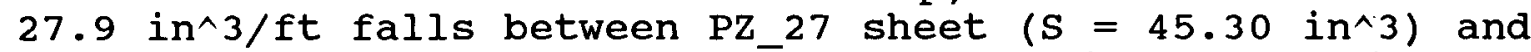
PDA_27 sheet $(S=14.30$ in^3). The first one will be selected and tried.

Note that if tie-back anchors were used, the piling would have to be sized for the downward force component of the anchors a combined stress situation.

\section{Soldier Piles}

Similarly, soldier piles could be designed for $12 \mathrm{ft}$ wide strip and $15 \mathrm{ft}$ (strut spacing) high for earth pressure. 


\section{A.3.2 Retaining System Members}

\section{Wales}

The procedure for sizing wales is:

1. Compute bending moment;

2. Pick trial section and compute bending stress;

3. Determine axial stress (wales may be axially loaded due to deflection of end retaining walls) and slenderness ratio $(\mathrm{Kl} / \mathrm{r})$ for both axes;

4. Select $F($ a) from AISC Column Tables;

5. Check effect of combined stress [Sect. 1.6.1-AISC]; and

6. Repeat steps 2 through 5 if necessary.

\section{8 truts}

To compute the reaction on a strut, multiply the lateral pressure by the load carrying area (12 ft by 15 ft spacing of struts):

1. Find the maximum reaction force on strut;

2. Try a WF section and determine slenderness ratio ( $1 / \mathrm{r})$ where $r=$ radius of gyration, and $l=$ the unbraced strut length;

3. Determine the allowable stress (Fa) from current AISC Column Tables; and

4. Repeat steps 2 and 3 until strut capacity $>=$ strut load

For maximum moment calculations, struts are assumed to be simply supported and axially loaded. struts or tie-back reactions are calculated assuming each support carries the load developed over half the distance to the next retaining reaction force position.

\section{A.3.3 Lagging}

Lumber may be surfaced on one side (SIS), two sides (S2S), two edges (S2E), a combination of sides and edges (SISIE, SIS2E, S2SE), or on all four sides (S4S, Hurd 1961). the length of lumber ordered, when this can be specified, should be such that it can be used to the best advantage with little waste. Most common lengths commercially available range up to $20-24 \mathrm{ft}$ in even-numbered increments of $2 \mathrm{ft}$.

Lagging Design: First, arching is assumed to take place in granular soils, and stiff to hard cohesive soils. Arching may not take place in soft cohesive soil, where the overburden pressure approaches four times the cohesive strength of the soil. The arching effect occurs when soil pressure causes lagging to deflect (timber overstress) and 
thus decrease overall lateral pressure. Therefore, arching may only be assumed under special conditions and for these full active lateral pressure must be accounted for. For CMSA, the maximum moment for lagging is computed as $(p * 1 \wedge 2 / 12)$ when arching is present (Ratay 1984).

Three criteria are used to check timber lagging design: maximum moments allowable, maximum deflection, and maximum shear. For the CMSA prototype, the first one was chosen as the criterion to select a section for lagging.

Moments $(M)=f * 8$

where $M$ is maximum moments (Resisting moments), $f$ is allowable stress in extreme fiber,

$S$ is section modulus $\left(b * \mathrm{H}^{\wedge} 2\right)$.

There are several commercial designations and species that are classified into groups is reported in CSA (CSA Standard 085-1976). For the sake of the prototype application, we adopt the Douglas-fir-larch species Identification, and Select structural grade (allowable bending stress of 1850 psi (see pp. 31 Table-7). Therefore, applying following formula:

$\mathrm{M}=\mathrm{f} * \mathrm{~s}$

where

$\begin{array}{ll}\mathrm{f} & =1850 \mathrm{psi}, \\ \mathrm{M} & =\mathrm{P} * \mathrm{~L}^{\wedge} 2 / 8 \text { for no arching, or } \\ \mathrm{M} & =\mathrm{P} * \mathrm{~L}^{\wedge} 2 / 12 \text { for Arching } \\ \mathrm{S} & =\mathrm{b} * \mathrm{~h}^{\wedge} 2 / 6\end{array}$

For 1 = Unbraced Lagging Length $=12 \mathrm{ft}$ (horizontal spacing of struts)

So, for $b=4$ in Nominal ( $31 / 2$ effective),

$\begin{array}{lll}\mathrm{S} & =\mathrm{M} / \mathrm{f} \\ \mathrm{b} * \mathrm{H}^{\wedge} 2 / 6 & =\mathrm{P} * \mathrm{~L}^{\wedge} 2 / 8 / 1850 \\ \mathrm{~b} * \mathrm{~h}^{\wedge} 2 & = & \mathrm{P} * 12^{\wedge} 2 * 6 /(8 * 1850) \\ \mathrm{b} * \mathrm{~h}^{\wedge} 2 & =\mathrm{P} * 0.058 \\ \text { For } \mathrm{b}= & 3.5 \mathrm{in}, \mathrm{h}=\operatorname{sqrt}(\mathrm{P} * 0.058 / 1.5) .\end{array}$

A data base for the lagging members used in the prototype is shown in table A.2. 
Member Designation

$$
\begin{array}{r}
24 \\
3-4 \\
4-4 \\
46 \\
6-6
\end{array}
$$

Section Modulus ( $\mathbf{b}^{\left.* h^{\wedge} 2\right)}$

(in^3)

3.06

5.10

7.15

17.65

27.7

Table A.2 Lagging Members 


\section{Appendix B}

\section{Pile Driving Production Derivation}

\section{B.1 Introduction}

This appendix is divided into two parts. The first one reviews basic methods and formulas for calculating soil resistance to pile driving. A formula for total driving time as implemented in CMSA is derived. The second part utilizes dynamic formulas for equating theoretical hammer energy with soil resistance in order to estimate the speed of pile driving.

It should be mentioned that the literature does not contain consistent relationships among soil, pile and hammer properties. The essence here is to adopt some of those formulas to develop our model for predicting pile driving progress rate. The adequacy, validity, and verification for these relationships are beyond the scope of this thesis.

\section{B.2 Soil/Pile Friction Calculations}

The following nomenclature is used.

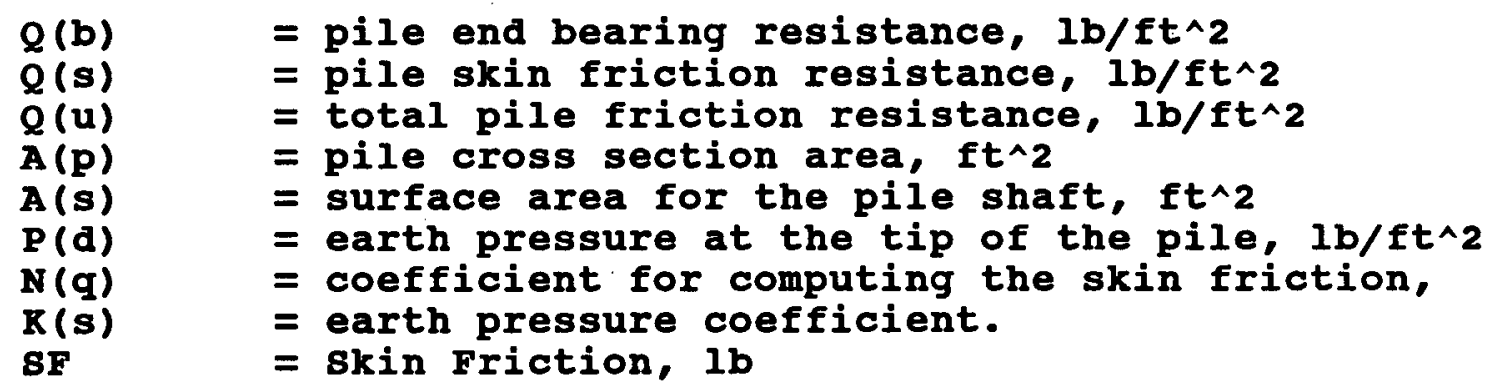

Several methods exist for soil resistance calculations in relation to pile driving. They include cone Landa, API (Toolan and Fox 1977); Cone Penetration Test (CPT), Standard Penetration Test (SPT) (Tomlinson 1975), and/or use of ultimate (upper bounds) unit skin and end bearing friction (Peurifoy 1970).

Soil, pile, and hammer interactions significantly govern the pace of pile driving. We start with a review of skin and end bearing formulas. SPT in conjunction with the ultimate bounds for soil resistance for the CMSA prototype. 
The basis of the "static" or soil mechanics method of calculating the ultimate carrying capacity of a pile is that the ultimate carrying capacity is equal to the sum of the ultimate resistance of the base of the pile and the ultimate skin friction over the embedded shaft length of the pile, i.e.:

$Q(u)=(Q(b)+Q(s)) / 2.5$

where

$$
\begin{array}{ll}
Q(b)= & \text { base resistance, } \\
Q(s)= & \text { shaft resistance, and } 2.5 \text { is a safety } \\
& \text { factor. }
\end{array}
$$

Knowing the angle of shearing resistance of the soil at the base level, $Q(b)$ can be calculated for different soil conditions as described below.

\section{B.2.1 Sands}

Total Base Resistance =

$$
Q(b)=A(p) * P(d) *(N(q)-1)
$$

where

$$
\begin{aligned}
& A(p)=\quad \text { Cross section area of the pile } \\
&= \begin{array}{l}
\text { Effective overburden Pressure at pile } \\
\text { base level }
\end{array} \\
&=\begin{array}{l}
\text { Bearing Capacity Factor that is relevant } \\
\text { to angle of shearing resistance of sand } \\
\text { and SPT in } N \text { blows } / 300 \mathrm{~mm} .
\end{array}
\end{aligned}
$$

Total skin Friction $=\mathbf{s F}$

$$
=K(s) * P(d) * \tan (\text { delta })
$$

where

$$
\begin{array}{lll}
\mathrm{K}(\mathrm{s}) & =\text { Earth Pressure Coefficient } \\
\text { Delta } & = & \text { Angle of Wall Friction }
\end{array}
$$

Therefore, SF force is = Unit skin Friction * Average effective pressure * Surface Area

where

$$
=\underset{\mathrm{A}(\mathrm{S})}{\mathrm{S})} \text { * Avg. } \mathrm{P}(\mathrm{d}) * \tan \text { (Delta) * }
$$


Avg. $P(d)$ is the average effective overburden pressure over the embedded depth of pile, and $A(s)$ is the embedded surface area.

Broms (Tomlinson 1975) has related values of $K(s)$ and delta to the effective angle of shearing resistance (phi) for various pile materials and relative densities as shown in table B.1 below:

$\begin{array}{llccc}\begin{array}{l}\text { Pile } \\ \text { Material }\end{array} & \begin{array}{c}\text { Low Density } \\ \text { (Loose Sand) }\end{array} & \begin{array}{l}\text { High Density } \\ \text { (Dense Sand) }\end{array} & \begin{array}{l}\text { Low Density } \\ \text { (Loose Sand) }\end{array} & \begin{array}{l}\text { K(s) } \\ \text { (Digh Density }\end{array} \\ \text { Steel } & 20 & 0.5 & 1.0 & 1.0 \\ \end{array}$

Table B.1 Values for Angle of Internal Friction [from Bowels 1977]

Unit skin friction, in a uniform cohesionless soil, increases linearly with increasing depth. A peak value of $2.30 \mathrm{ksf}$ for unit skin friction is used for straight-sided piles. Where piles are driven deeper than 20 times their diameters, rule of thumb values can be used for the average skin friction over the whole shaft. Table B.2 relates the sand soil density to an upper bound of skin friction based on experimentation.

Relative Density

$<0.35$ (loose)

0.35 - 0.65 (medium dense)

$0.65-0.85$ (dense)

$>0.85$ (Very dense)
Average Skin Friction

$0.209 \mathrm{ksf}$

$0.21-0.52 \mathrm{ksf}$

$0.52-1.46 \mathrm{ksf}$

$1.46 \mathrm{ksf}-2.30 \mathrm{ksf}$

Table B.2 Ultimate skin Friction for sands

[from Bowels 1977]

\section{B.2.2 Clays and silts}

End Resistance:

where

$$
\mathrm{Qb}=\mathrm{NC} * \mathrm{Cb} * \mathrm{Ab}
$$


$\mathrm{Nc}=9$ if pile driven more than 5 diameters

$\mathrm{Cb}=$ undisturbed shear strength

$\mathrm{Ab}=$ Cross section area

Skin Resistance (1):

$$
\text { Qs }=\text { alpha } * C d * \text { As }
$$

where

alpha $=$ adhesion factor. It varies for piles of the same type on the same site between 0.4 to 1.0 for clays of $1.50 \mathrm{ksf}$ shear strength, and between 0.25 and 0.45 for clays of 2.58 to $3.1 \mathrm{ksf}$ shear strength (Ratay 1984).

Cd = undisturbed shear strength adjacent to the shaft,

As $=$ surface area of the shaft

Meyerhof (Tomlinson 1975) states that a maximum skin friction of $1.13 \mathrm{ksf}$ (calculated on all surfaces of flanges and web) should be used for $\mathrm{H}$ piles.

Factors such as pore pressure (water may work as lubricant), ground heave (and re-consolidation), lateral vibration, smearing, and "strain-softening" are of significance but may vary in its effect even on the same site for adhesion computation for clays.

Ultimate loads on piles driven into cohesive soils are as follow:

For end resistance,

$$
Q(b)=N(c) * C(b) * A(b)
$$

where

$$
\begin{array}{ll}
\mathrm{N}(\mathrm{c}) & =\text { bearing capacity factor } \\
\mathrm{C}(\mathrm{b}) & =\text { Shear strength at the base of the pile } \\
\mathrm{A}(\mathrm{b}) & =\text { Cross section Area of the pile tip. }
\end{array}
$$

For skin friction, $Q(s)$

$$
=\text { alpha *Avg. } C(b) * A(s)
$$


where,

\begin{tabular}{|c|c|c|}
\hline alpha 13 & $=$ & adhesion factor. \\
\hline Avg. C (b) & $=$ & $\begin{array}{l}\text { average undisturbed shear strength of } \\
\text { the clay adjacent to the shaft }\end{array}$ \\
\hline$A(s)$ & $=$ & Surface area of the shaft. \\
\hline
\end{tabular}

For uniform clays, the shear strength increases with depth. The average value of shear strength over the whole shaft length is taken for $\mathrm{c}(\mathrm{b})$. When clays exist in layers, e.g. soft clay over stiff clay, the skin friction is computed for each layer using the corresponding adhesion factor appropriate to the shear strength and overburden conditions.

\section{B.2.3 Time Required for Driving a sheet pile}

A relationship is required to estimate production rate of pile driving as a function of soil/pile/hammer interaction. Dynamic formulas and more exact methods such as weave Equation Analysis for piles that fit a class of impact hammers have been developed (Peurifoy 1970, WEAP 1983). No relationships are available so far for vibratory hammers. suppose that a soil/hammer/pile scenario is given and we want to know production rate for a single steel sheet pile (or segments of piles) to be driven to refusal depth. Driving time is divided into two components: variable time and fixed time. The first counts for the time when the pile driver is in driving mode. The latter counts for the time when the crane is used to seat the pile, the crew is fitting the hammer on the pile, the crane moves from one place to another, and so forth.

Thus,

Production Rate per single steel sheet Pile or soldier Pile $=$ Fixed Time + variable Time (B.8)

This is based on the assumption that the crane is dedicated to driving one sheet pile at a time. That implies that both hammer and crane are tied up together. Often the driving pattern follows a wave. Adjacent piles are driven for short distances and at equal intervals and alignments.

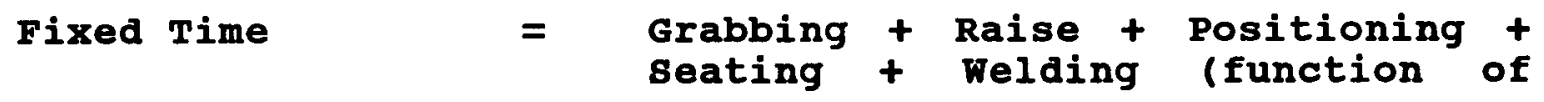

${ }^{13}$ Adhesion factors are calculated for three scenarios (Winterkorn and Fang 1975):

1. For sands or sand gravels over stiff clays;

2. for soft clay over stiff clays; and

3. for stiff clay. 
Number of vertical pieces of

Average Fixed Time installed sheet pile)

per one Pile $=n *$ (Grab + Raise + Position) + (n

- 1) * (Welding + seating)

in which $\mathrm{n}$ is the number of segmented or spliced pile

lengths. Segments of steel sheet piles may come in

different lengths of $40 \mathrm{ft}, 50 \mathrm{ft}, 60 \mathrm{ft}$ up to $80 \mathrm{ft}$

depending on mode of transportation, depth of excavation, project site restriction, crane and hammer available, and existing pile stock for the contractor. Therefore,

$\mathrm{n}$

$=$ Excavation Depth / Length of Sheet Piles) - 1 (if first term in not integer)

Total Number of Pile segments

$=1 c$ * Number of Pile Driving cycles.

... (B.10)

where lc is the pile segment length.

Variable Time per one cycle of Pile Driving

$=$ Production Time (Driving Mode)
$=\quad$ unction of (pile segment length,
soil condition, Tunnel Depth, sheet
pile type, Pile Driver type)
-.. (B.11)

The derivation for this component is treated in the next section where dynamic formulas are employed.

Number of Pile Driving Cycles

$=$ Tunnel Length $* 2 * 12$ / One Pile ... (B.12)

where one Pile Installation Width denotes one unit width of pile installation, i.e., there might be (n) pile segments (say 2 to $31 / 2$ segments) installed for one unit wall width (say one foot) for one face of the excavation ( 2 for two faces, and 12 to convert feet into inches when the pile width is specified in inches).

Total Pile Driving Activity Time

$$
=\begin{aligned}
& \text { ((Fixed Time per One Cycle) } \\
& \text { (Variable Time per One Cycle)) * } \\
& \text { Number of Pile Driving Cycles. }
\end{aligned}
$$

Assuming 8 working hours per days (could be variable if desired), then 
Number of Working Days

(Total Time / 8) days

(B. 13)

\section{B.3 Pile Driving Production Rate Estimation}

\section{B.3.1 Pile Driving Production Formula Derivation}

The author developed the production rate formula from consideration of first principles. With minor variations, a similar analysis was described in Gates and Scarpa (1984).

The following nomenclature is used:

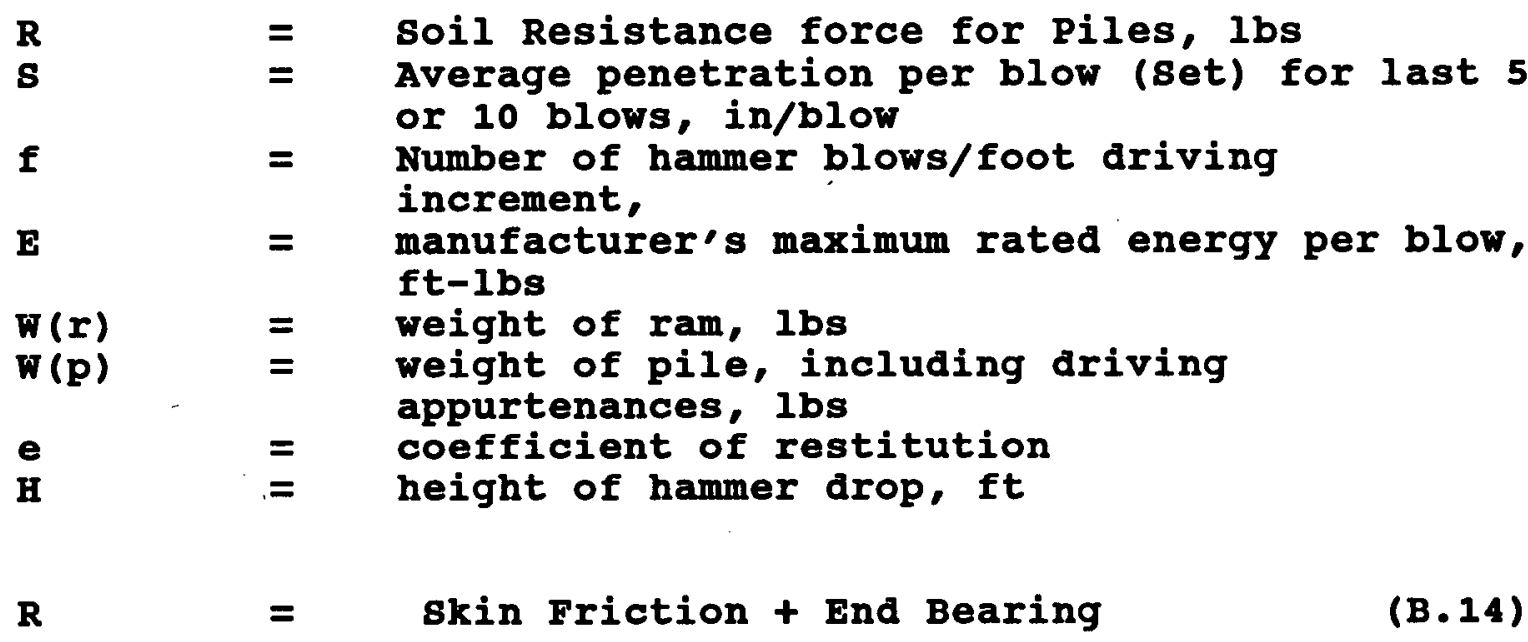

For non-displacement piles of steel sheet piles and soldiers piles, end bearing can be ignored. Therefore,

$\mathbf{R}$

$=\quad$ skin Friction

In theory, theoretical energy delivered by the impact hammer is equated to soil resistance during pile driving. The relationships expressed by dynamic formulas, empirically derived, relate displacement of sheet pile (S) to hammer delivered energy and soil resistance. For each class and type of impact hammer, there is a dynamic formula.

For a drop hammer, the formula is

$$
\text { R } \quad 2 * W * H+0.10
$$

For single acting air/steam hammer, the dynamic formula is

$$
\begin{array}{ll}
\mathbf{R} & =2 * W * H / \mathrm{B}_{1} \\
\mathrm{~s}(\mathrm{i}) & =(2 * W * H / \mathrm{R}(\mathrm{i}))-1
\end{array}
$$


in which $R(i)$ and $S(i)$ are incremental soil resistance and corresponding pile penetration rate per unit depth (in ft). Equations B.16 and B.17 are based on Engineering News Formula (Peurifoy 1970).

An alternate dynamic formula for drop hammer (as well as other hammers) based on Highway Department Modified Engineering News Formula (Peurifoy 1970) is:

$\begin{aligned} & R=(2.5 * E(s a m e \text { as } W * H)) \\ & /(W(r)+W(p))(W(r)+e * * 2 * W(p)) \\ &(B .18)\end{aligned}$

in which $\mathrm{E}=\mathrm{W} * \mathrm{H}$ (potential energy with no losses). Similarly, the Modified Highway Department Modified Engineering News Formula for single acting air/steam hammer is:

$R(s)=((2 *(W+A(p)) * H) /(s+.10 * P / W)$

where,

$\begin{array}{ll}\mathrm{A}(\mathrm{p}) & =\text { Area of the piston of the ram } \\ \mathrm{P} & =\text { Air or steam pressure on the piston. }\end{array}$

The hammer theoretical energy, $E=W \star H$, is used in the equations. The net hammer energy could be less ( $80 \%-20 \%)$ than the theoretical energy due to hammer energy losses (impact, friction, etc.) and hammer efficiency.

Many dynamic formulas have been developed. Recently, Wave Equation Analysis for Piles (WEAP) has emerged as more accurate than dynamic formulas (WEAP 1984). However, WEAP programs are quite big, complex, restricted to a class of diesel hammer, and requires an intelligent front end and back end for automation. Therefore, for the CMSA prototype, the Engineering News dynamic formulas were adopted for ease of understanding, coding, and interfacing. An assumption adopted is that there are no energy losses, and the hammer efficiency is specified by the user.

Thus, the basic form of the equation adopted for predicting $R$, the soil resistance for piles is of the form:

\section{R = Unit skin friction (at depth z) * Pile surface Area}

$R$ can be approximated using interpolation at different depths, $R$ at depth $z$, using a static formula is: 


$\begin{array}{lll}\mathbf{R} & = & \text { skin friction (at z depth) * ssp-surface } \\ \mathbf{R}(1) & = & \text { area/12 } \\ \text { skin friction (at depth } \mathrm{z}(1)) * \text { ssp-surf. } \\ \mathbf{R}(i) & =\text { area/12, } \\ \mathbf{R}(i) & =\mathrm{fsp} \text { (depth } \mathrm{z}(\mathrm{i}))\end{array}$

SSP-surface area corresponds to the surface area of any nondisplacement piles (e.g. steel sheet piles, $H$ or soldier piles). Skin friction increases with depth and thus rate of change can be emulated as a step function or linear relationship versus depth. For CMSA, it is assumed to be linear and the analysis is performed in 1 foot increments.

$R$ at a depth interval of every $x$ feet (say one foot), and using equation B.17 is:

$s(i)=(2 * W * H / R(i))-1.0$

For each foot depth interval (z(i)-1), duration (T(i)) for driving pile one foot form the surface can be approximated as follows:

T (i) $=$ (x foot * 12 in/foot) $/((s(i)$ in inches/blow) * $f$ (blows/minutes)) in minutes

(B.23)

Therefore, the total variable time per pile driving cycle is:

Variable Time $\quad=\quad$ sum $(T(i))$

$$
=f(R(i), s(i), d(z))
$$

Once the variable time component value is computed in the Drive.C routine, it is passed to the CMSA prototype which then adds it to the fixed time component to obtain the total pile driving time (see figure 4.10 of Drive.c routine interface with CMSA).

To show the relationship between the number of blows it takes the a hammer to drive a pile $1 \mathrm{ft}$ and the soil resistance at time of driving (SRD), figure B.1 (a) presents such a profile for a displacement pile. Figure B.1 (b) shows the expected blow count/depth relationship for a particular combination of driving plant for predicting initial penetration of jackets below the sea bed in normally consolidated clays (Toolan and Fox 1977). These curves are predicted based on semi-empirical equations which are then considered during wave equation analysis computation.

Although figure B.1 is for an offshore displacement pile, there is a parallel for non-displacement piles installation 
behavior. For instance, hammer efficiency influences hammer performance where hammer blow count varies with driving depth and soil resistance.

Blow count: blows/ft

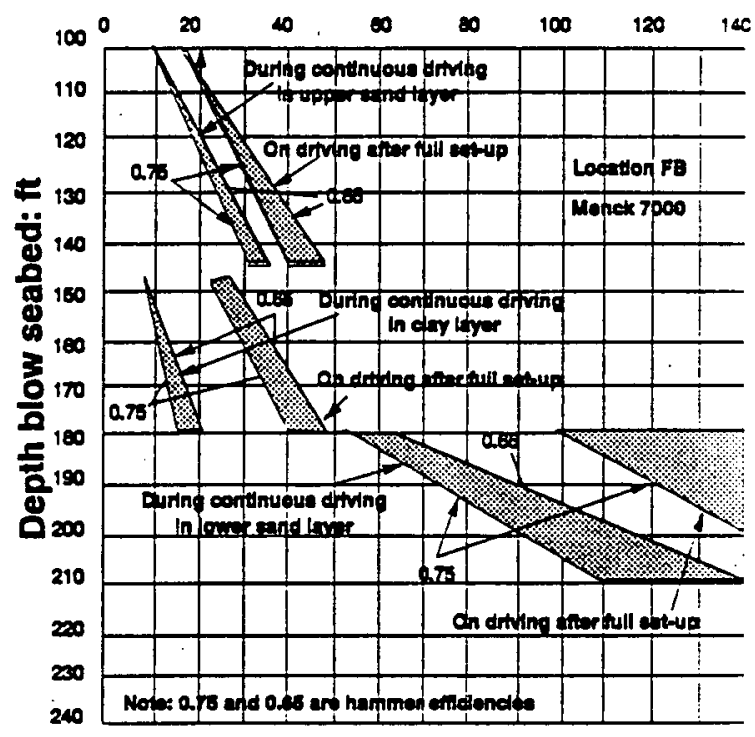

(a) Blow Count/Resistance Curves

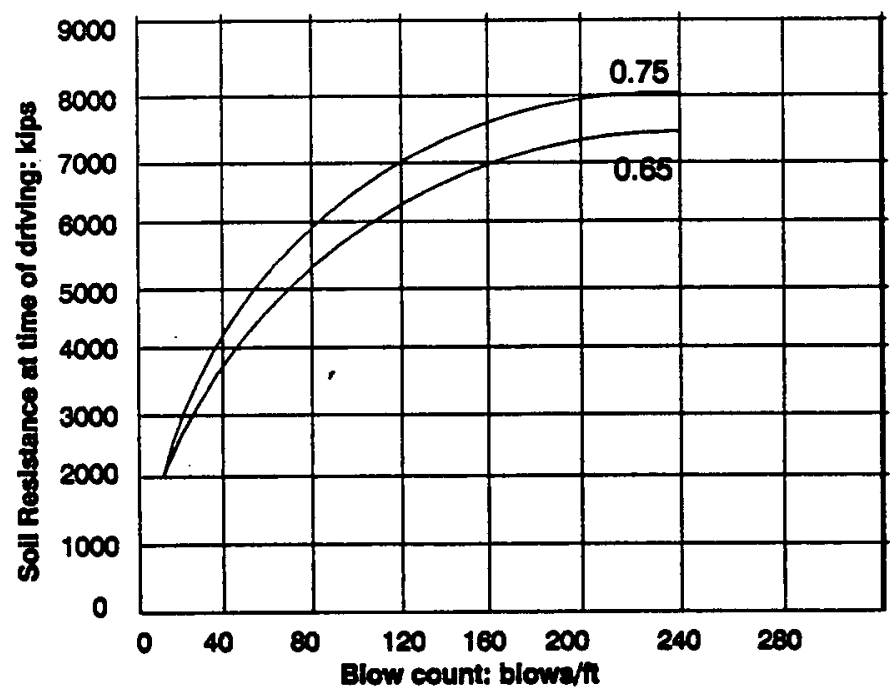

Plle Driving Information Kor

Piv

Ponerretion

Arrvity Connetore

Plo segment Length

Pllo tip in

Manmer

Cuncilon

Spring conetant anvil

Coct. of reatruttion

Enllendere

Total aldo radatanes

total polint realstanos

Computer run
: 54 th. dia, open plpo.

$: 200 \mathrm{n}(603 \mathrm{~m})$

$: 2$ by $13 \pi$

$: 3 \mathrm{~m}$

: Sand

: Menck 7000. 1 segment

: 12 herd wood

$: 17500 \mathrm{NJ} / \mathrm{cm}$

$: 0.78$

$: 0.66,0.75$

: 1875 kpes (850 in)

: varying

: $750308 / 3$

(b) Predicted Blow count/Depth, 54 in dia. Piles

\section{Figure B.1 Hammer Blow Count versus soil Resistance [from Toolan and Fox 1977]}




\section{B.3.2 Example}

A proposed tunnel of $1200 \mathrm{ft}$ long, $66 \mathrm{ft}$ deep, $20 \mathrm{ft}$ wide is to be constructed in a remote area. The soil profile consists of two layers: 40 feet of loose sand layer on top of stiff clay.

After running CMSA, a steel sheet pile alternative was selected for consideration. Piles properties relevant to the Drive.c routine are:

- Sheet Pile Designation is PZ_27 (ASTM A36)

- PZ_27 Cross Section Area is $\overline{1} 1.91$ in^2, and

- PZ_27 Surface Area is $4.94 \mathrm{ft}^{\wedge} 2 / \mathrm{ft}$ (SA).

other properties such as cross section area are used for setting an upper bound for hammer energy without damaging the pile while driving. As well, it is used in another variation of the Drive routine when SPT is used to interpret the soil properties.

CMSA, based on project context, further specifies a hammer utilizing its construction and geotechnical knowledge. Properties that apply to the Drive.c routine are:

- Hammer_Type is Double Acting Air Hammer (DAAH),

- Hammer Frequency is 95 cycles/min, and

- Rated Hammer Energy is 11,490 1b-ft.

other properties, such as hammer ram weight are used to indirectly select a suitable crane and recommend a pile segment length, if required. In order to pass the parameters to Drive.c routine, CMSA creates two NExpert flat (ASCII files) data bases. The first one contains the soil profile and is called "soil.nxp" and has this format:

$\backslash$ Soil_type_1\"Loose_sand"

$\backslash \operatorname{star} \bar{t} 1 \backslash \bar{I}_{1}$

/* start pile driving at depth 1 ft

$\backslash$ Finish_1\ "40"

$\backslash$ soil_tȳpe_2\"stiff_clay"

$\backslash$ start_2\" $41 "$

/* Stiff clay starts at

$\backslash$ Finish_2\"71" depth $41 \mathrm{ft}$ * /* Refusal depth is $71 \mathrm{ft.*/}$ 
The second file, "Hammer.nxp", includes the hammer and sheet pile relevant properties.

$\backslash$ Refusal_Depth \"71"

$\backslash$ Frequency \"95"

$\backslash$ Energy $\backslash " 11490 "$

$\backslash$ Surface area \"4.94"

\ Hammer Typel "DAAH"

$/ * \quad f t \wedge 2 / f t$

$\backslash$ Hammer_Model\"Vulcan of 11_3B"

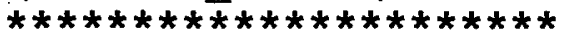

Note that the sheet pile should be driven 5 ft below excavation level $(5+66=71 \mathrm{ft}$ deep) to prevent or minimize soil heave for clays and soil boiling for sands.

There are two output files of "Out. nxp" and "out.out". The first one is retrieved by CMSA to interpret and analyze selected variable values. The second file, "out.out" contain more details about the same run session. A sample of "Out.nxp" is shown below:

$\backslash$ Incremental Depth.amount $\backslash=" 1.00 "$

$\backslash$ Depth.amount $\backslash=" 71.00 "$

$\backslash$ Soil_Resistance.amount $\backslash=" 33188.82 "$

$\backslash$ Penetration . amount $\backslash=" 0.8039 "$

\Incremental_Time.amount $\backslash=" 0.30 "$

\Variable_Time.amount $\backslash=" 8.49 " /$ * Cumulative variable Time */ $\backslash$ TechnicaI_Feasibility.state $\backslash=" T r u e "$

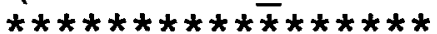

A sample of "Out.out" results is shown in table B.3. Figure B.2. presents the selected hammer (DAAH, efficiency 71\%) blow count versus driving depth. 
Appendix B. Pile Driving Production Derivation

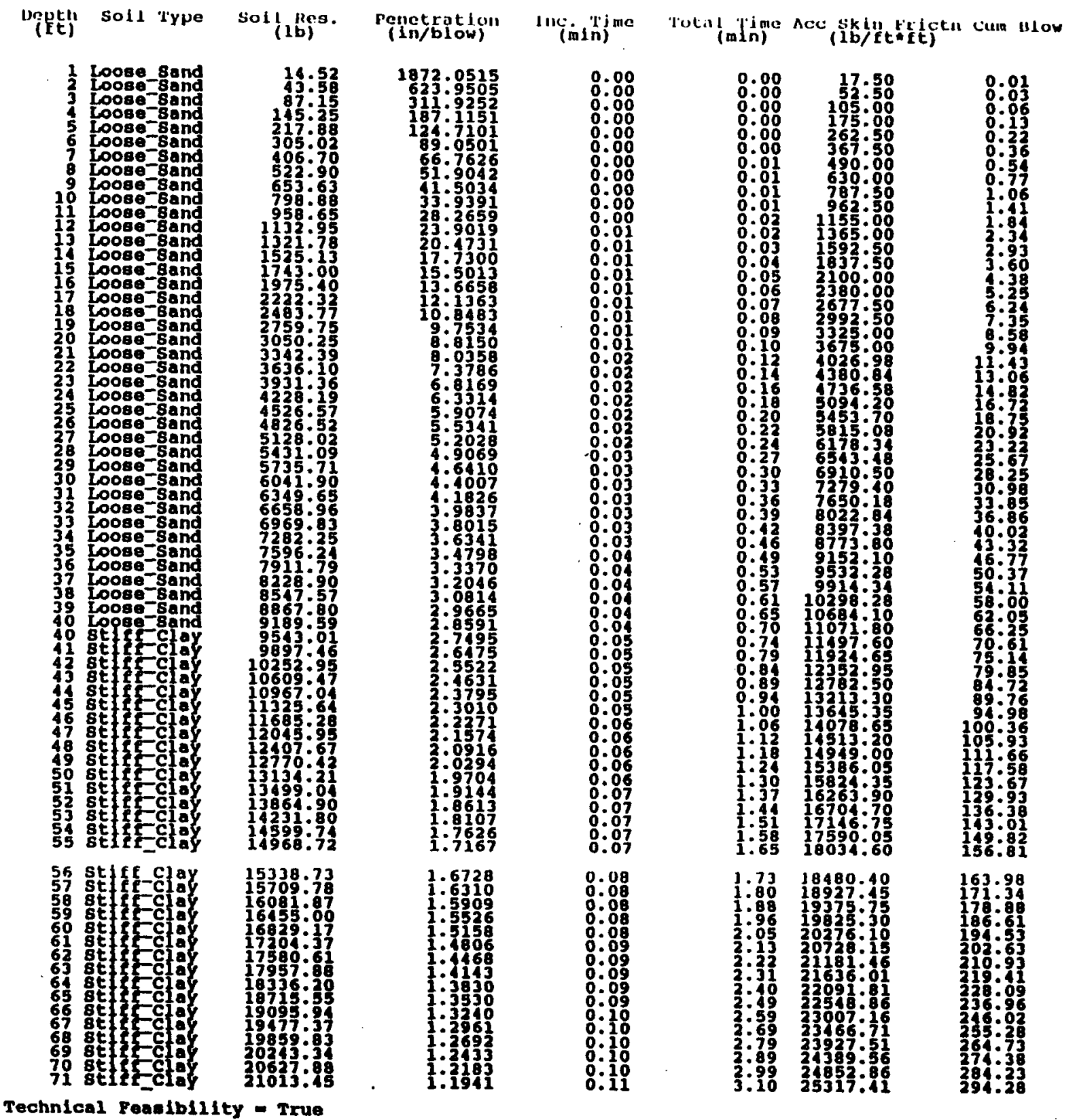

Table B.3 "Out.out" sample Output for Example Problem 


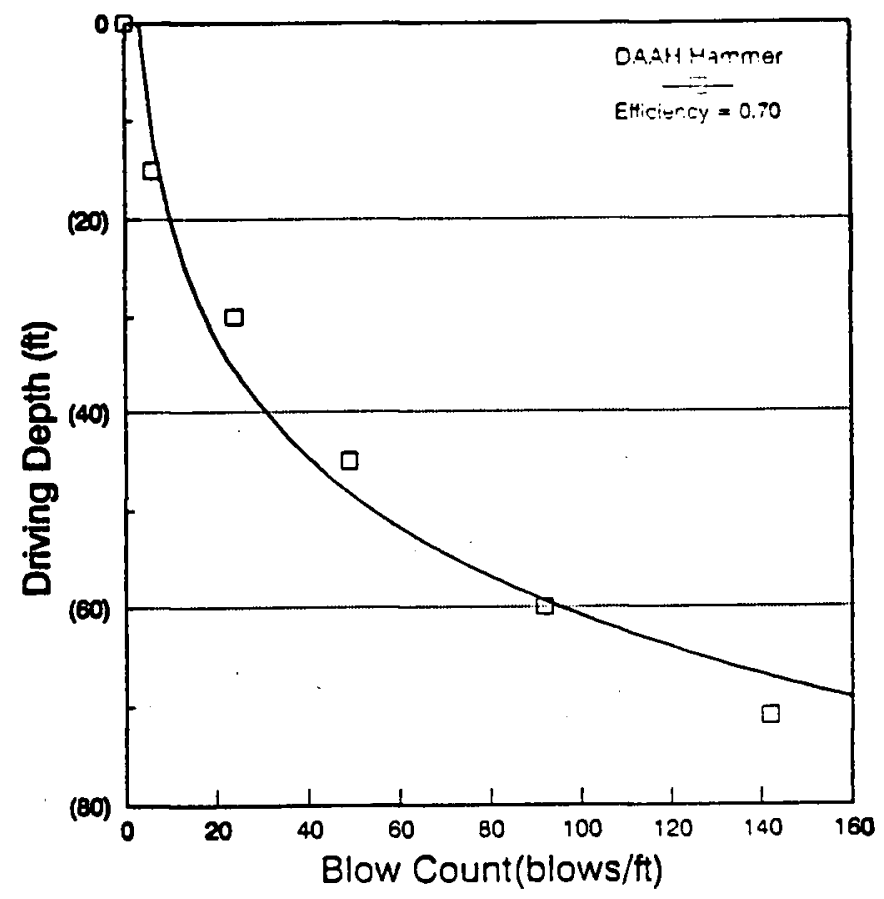

\section{Figure B.2 . Hammer Blow Count versus Driving Depth}

sample Run output Variable Interpretation: The output file "Out.out" contains most of the variables that are produced by the program "Drive.c" and interpreted by CMSA. The variable time, based on equation B.24, for one pile driving cycle is 33.21 minutes as printed at the last driven soil step. Note the high speed of pile driving, approximately 22 $\mathrm{ft} / \mathrm{blow}$, on the top part of the soil layer (table B.1). The reason for this high value is due to near zero resistance of the sand for the first driving foot resulting in very small incremental time (rounded to zero for the first four feet). practically, the steel sheet pile by its own weight sinks into the sand for few feet before using the pile driver. So far, no adjustment has been made to account for this duration.

The technical feasibility state returns a Boolean value of "True" thus implies that the pile can be driven to refusal depth successfully without a stoppage due to high resistance or obstacle (by observing blow count during driving), and that the number of hammer blows per minute did not exceed its upper bound rule of thumb for pile damageability).

From figure B.2, as the depth increases, the cumulative soil resistance to pile driving increases, and thus a higher blow count is required to drive the pile further. The blow count is plotted for every $10 \mathrm{ft}$ of soil depth. In this "Drive.c" routine, the blow count is found as the inverse of 
penetration rate (in/blow) for the last inch in each driven foot which is then multiplied by 12 in/ft to convert blow count to blows/ft. For example, at soil depth $20 \mathrm{ft}$, the 20 th foot blow count (in/blow) which is divided into 12 in/ft yields $10 \mathrm{blow} / \mathrm{ft}$. This value is the incremental blow count at this depth.

\section{B.3.3 Drive.c Program}

Figure B.3 presents a flow chart for the "Drive.c" routine written in $C$ programming language. This routine reads input data from two input files of "Hammer.nxp" and "Soil.nxp". These files are created by the CMSA prototype which contains relevant information regarding hammer/soil/pile session scenario. The routine returns output to two data files of "Out.out" and "Out.nxp". The first contains detailed output variables and the second file contains selected output variables. The latter then is retrieved by the prototype CMSA for further information processing.

The routine "Drive.c" function is to compute the cumulative variable time component based on dynamic formulas as derived in B.14 to B.25. To do so, at every foot from surface to the refusal depth during pile driving (see table B.1), computations for the output variables are done. These variables include soil resistance, pile penetration rate, incremental and cumulative variable time. 


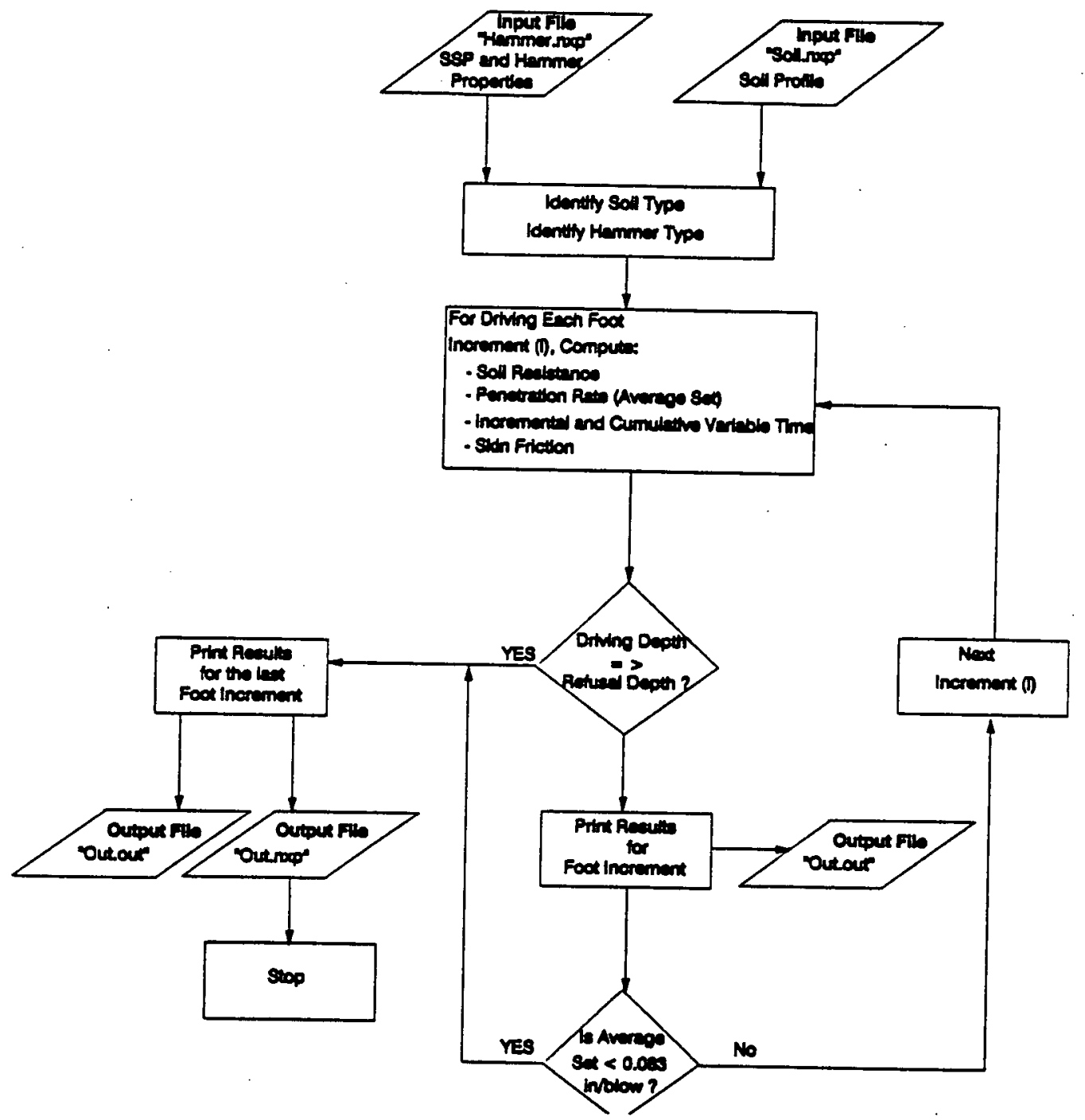

Figure B.3 Drive.c Routine Flow Chart

Figure B.4 sums the progression of developing the model as finalized in equation B.15. Part B.4 (a) shows the forces applied at the pile in equilibrium, part (b) presents soil resistance, and part (c) presents penetration rate and cumulative variable driving time.

If the unit depth, in part (b), is assumed to be $1 \mathrm{ft}$, then total soil resistance is computed by summing the area under the incremental resistance area. For part (c), penetration rate is computed at the last inch for each foot and held constant along this foot.

The $\mathrm{C}$ source code for Drive.C is given in listing B.1. 


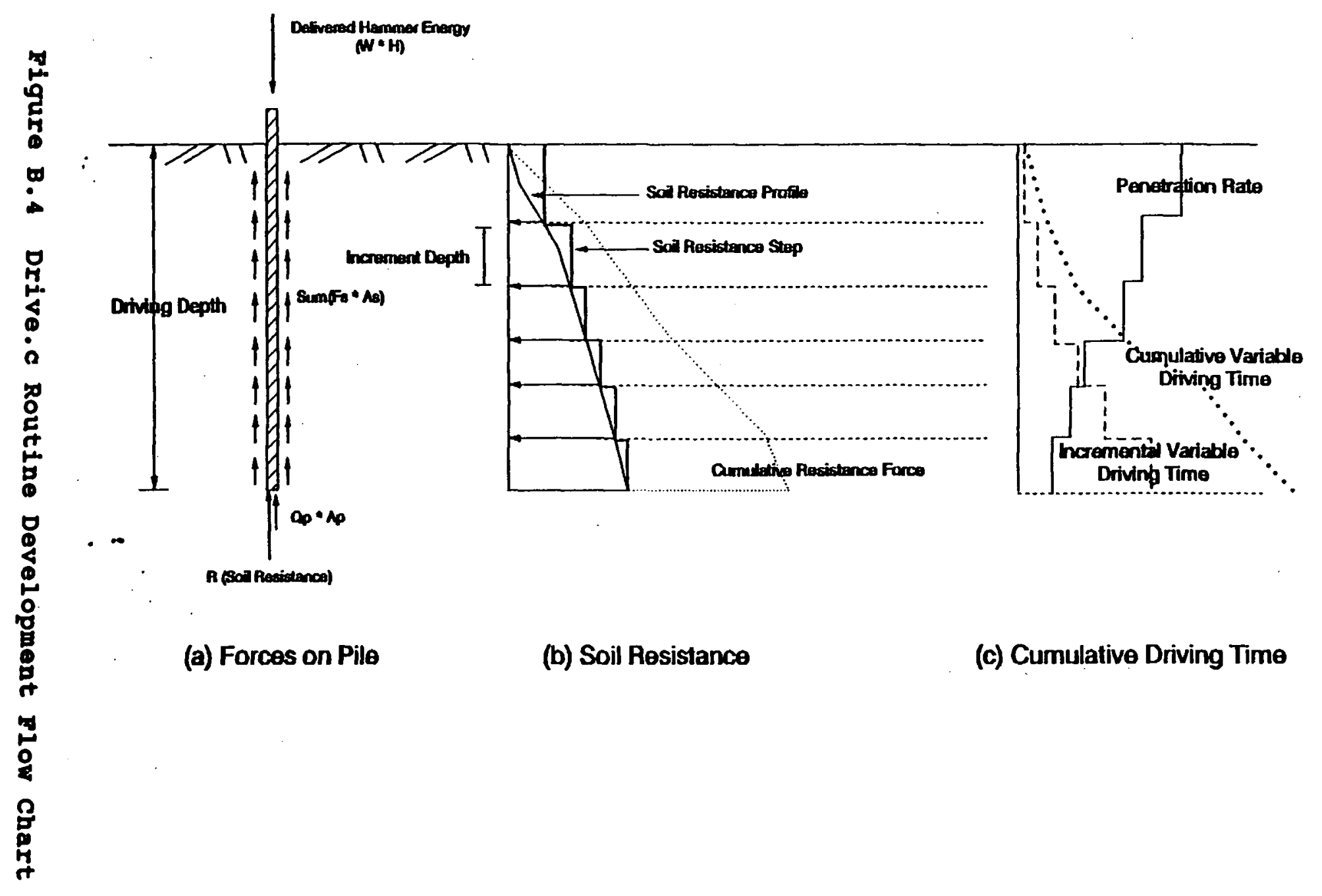


\#include < math.h>

\#include < stdio.h $>$

\#include $<$ fcntl.h $>$

\#include <io.h $>$

\#include < float.h $>$

\#include <sys \types.h>

\#define BUFFSIZE 1000

\#define STRSIZE 20

$/^{*}$ This program calculates the total time required to drive pile length without splicing. The data accepted from input file are the following:
1. L
2. Depth
$=\quad$ Total depth of pile driving, $\mathrm{ft}$
3. SA
$=$
Depth as a variable to count for a unit step, $\mathrm{ft}$
4. E
$=\quad$ Surface Area, in^2
5. F
$=\quad$ Energy, or $\left(\mathrm{W}^{*} \mathrm{H}\right), \mathrm{lb}-\mathrm{ft}$
6. $\mathrm{T}(\mathrm{i})$
$=\quad$ Hammer Frequency, cycles (blows)/ minute
7. Variable'Time
$=\quad$ Incremental time per depth step (10 foot here). $\mathrm{min}$
$=$
Sum of incremental times (total time for driving a total length of $L$, minutes
8. R(i)
9. S(i)
Soil resistance for piles during driving (static or dynamic), lb
Average penetration per blow (set) for last 5 or 10 blows, in/blow
10. delta_d most of the required variables above, in this routine it is set to 1 foot
Increment or Unit Step of Driving Depth for Calculating

\section{$/^{*}$ Program Assumptions}

It is important to emphasize that piles are used as earth retaining system rather than foundation support (for sheet piles and $\mathrm{H}$-piles).

1. Hammer/soil/pile is mimicked in this program.

2. Hammers denote impact type only. There are several hammers of Single Acting Air/Steam Hammer, Double Acting Air/Steam Hammer. Others will be added as required.

3. Soil stratum can be one or two layers. This description can be extended to more than that.

4. By equating total resistance to total allowable driving load, safety factors are not considered.

5. Skin friction (SF), is estimated bu using the linear relationship with depth set out in Peurifoy (1970). However, for speciality contractors, soil/pile/hammer scenarios are documented or recorded based on dynamic testing and/or from previous jobs.

6. The equation used does not consider end bearing resistance. However, in dense sand or stiff clay, this is a major consideration. This assumption is relaxed in a later stage.

7. A soil plug could may arise for H-piles driven in dense sand or soft/hard clay. It has not been accounted for. 
8. Energy losses such as hammer loss, impact loss, compression loss are not considered. However, for simplification, this may be accounted for in the hammer efficiency enquiry for the user in the CMSA before running this routine.

$* 1$

$/^{*} \quad$ Following soil profiles are assumed under the conditions that each skin friction is given by Peurifoy (1970). Thus no elicitation is required for unit weights, water table, and other soil parameters */

char *soil[] = \{ "Loose_Sand",

"Dense Sand",

"Soft_Clay",

\};

"Stiff_Clay"

$/^{*}$ Hammers provided are those of Single Acting Air/Steam hammers, Drop Hammers,

and Double Acting Air/Steam Hammers

*/

char *hammer[] = \{ "SAAH",

"DAAH" /* Double Acting Air Hammer */

"DASH",

"DH"

\};

/* Drop hammer */

main 0

\{

float SA, E, F, L, start, finish, AcSf, n_blows;

float depth, delta_d, Ri, Si, Ti, Variable_Time;

int $\mathrm{fd}, \mathrm{i}$, done;

FILE *f1, *f2;

char buffer[BUFFSIZE], valuestr[STRSIZE], c, scr_out;

char *bufferptr, *p;

char Soil_Type[STRSIZE + 1], Hammer_Type[STRSIZE + 1];

int NSOĪLS = sizeof(soil)/sizeof(char ${ }^{\overline{ }}$ );

int NHAMMS = sizeof(hammer $) / \operatorname{sizeof}\left(\right.$ char $\left.^{*}\right)$;

float SF (), HF O;

$/^{*}$ Read data from "Hammer.nxp" file which includes refusal depth (L), Hammer Frequency,

Hammer Energy, Pile Surface Area, and Hammer Type; in total five parameters

if $((\mathrm{fd}=$ open ("HAMMER.NXP", O_RDONLY)) = = -1)

return $(0)$;

read (fd, buffer, BUFFSIZE);

close (fd);

$/{ }^{*}$ Extract the values out of the buffer */

bufferptr $=$ \&buffer[0];

$\mathrm{i}=0$;

Listing B.1 Drive.c Routine for Pile Driving (continued) 


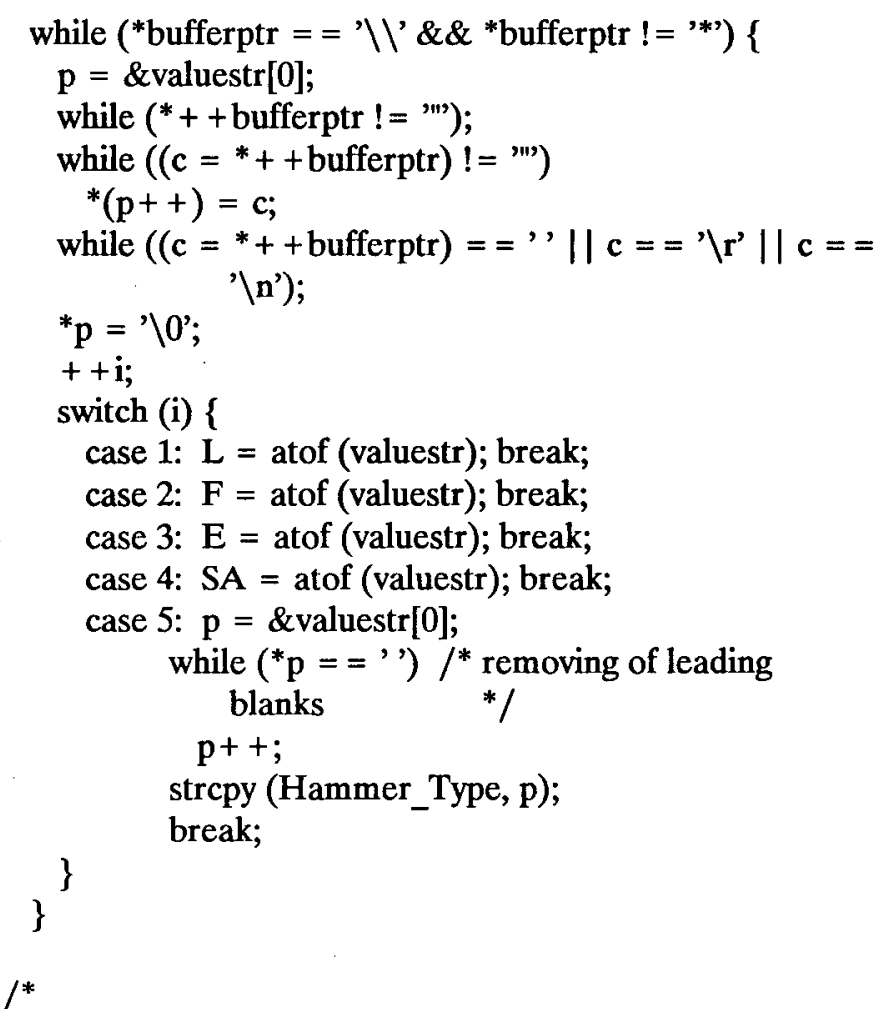

Files Called and Created for Input/Output

1. Input data are entered in two flat files with an .nxp extension to facilitate NExpert Object interface. These files are

- $\quad$ soil.nxp that contains the soil profile (soil type, start and end of each layer).

- Hammer.nxp that contains selected hammer and pile parameters.

2. Output results are shown in two files of (nxp) format. These are:

out.nxp, mainly used to retrieve selected output variables, especially technical feasibility state and cumulative variable pile driving time, for further knowledge processing and manipulation.

out.out, mainly used to check the validity of the output variables during development under the Dos. It contains incremental and cumulative values for soil depth, skin friction, penetration rate, time, and number of blows.

if $((\mathrm{fd}=$ open ("SOIL.NXP", O_RDONLY)) $==-1$ ) return (0);

read (fd, buffer, BUFFSIZE);

close (fd);

$/^{*}$ Open the "OUT.NXP" and "OUT.OUT" files to output results 


$$
\begin{aligned}
& \text { if }((f 1=\text { fopen ("OUT.NXP", "w")) }==\text { NULL) } \\
& \text { return (0); } \\
& \text { if }((\mathrm{f} 2=\text { fopen ("OUT.OUT", "w")) }==\text { NULL) } \\
& \text { return }(0) ;
\end{aligned}
$$

$/^{*} \quad$ Ask the user if he wants to see the output (out.out) on the screen or Not

$$
\text { */ }
$$

printf ("Do you want output on screen? (Y/N) ");

scr out $=($ char $)$ getchar () ;

fprintf (f2, " Depth Soil Type Soil Res. Penetration Inc. Time Total Time Acc Skin Frictn

Cum Blow $\left.\backslash \mathbf{n}^{\prime \prime}\right)$;
fprintf (f2," (ft)
(lb) (in/blow)
(min) (min)

$\left.(\mathrm{lb} / \mathrm{ft} * \mathrm{ft}) \backslash \mathrm{n}^{\prime \prime}\right)$;

if (scr_out $==$ ' $y$ ' ||$s c r$ out $==$ 'Y') \{ printf (" Depth Soil Type Soil Res. Penetration

Inc. Time Total

Time $\backslash \mathbf{n} ")$;
printf (" (ft)
(lb) (in/blow)
$\left.\left.(\min ) \quad(\min ) \backslash \mathrm{n}^{\prime \prime}\right) ; \quad\right\}$

Variable Time $=0.0$;

delta_d $=1.0 ;$ done $=0 ;$ AcSf $=0.0 ;$ n_blows $=0.0$;

$/$ * Extract the soil profile information out of the buffer */

bufferptr $=$ \&buffer[0];

while ('bufferptr $==$ ' $\backslash \backslash$ ' \& \& *bufferptr ! $\left.{ }^{*}{ }^{*}\right)$ )

$/{ }^{*}$ Get the soil type of this layer */

$\mathrm{p}=$ \&valuestr[0];

while $\left({ }^{*}++\right.$ bufferptr $\left.!={ }^{\prime \prime \prime}\right)$;

while $\left(\left(\mathrm{c}={ }^{*}++\right.\right.$ bufferptr $\left.) !={ }^{\prime \prime \prime}\right)$

$*(\mathrm{p}++)=\mathrm{c}$;

while $\left(\left(\mathrm{c}={ }^{*}++\right.\right.$ bufferptr $)==$ ', $\left\|\mathrm{c}=={ }^{\prime} \backslash \mathrm{r}^{\prime}\right\| \mathrm{c}==$

'\n');

${ }^{*} \mathrm{p}=$ ' $\backslash 0$ ';

$\mathrm{p}=$ \&valuestr $[0]$

while (* $\mathrm{p}==, ') /{ }^{*}$ removing of leading blanks */

$$
\mathrm{p}++ \text {; }
$$

strcpy (Soil_Type, p);

$/^{*}$ Get the starting depth of this layer */

$\mathrm{p}=$ \&valuestr[0];

while $\left(*++\right.$ bufferptr $\left.!={ }^{\prime \prime}\right)$;

while $\left(\left(\mathrm{c}={ }^{*}++\right.\right.$ bufferptr $\left.) !={ }^{\prime \prime \prime}\right)$

$*(\mathrm{p}+\mathrm{+})=\mathrm{c}$;

while $\left(\left(c={ }^{*}++\right.\right.$ bufferptr $)=={ }^{\prime},|| c==' \backslash r^{\prime} \| c=$

${ }^{*} \mathrm{p}={ }^{\prime} \backslash 0$;

start = atof (valuestr);

$/{ }^{*}$ Get the ending depth of this layer * $/$

$\mathrm{p}=$ \&valuestr[0];

while $\left({ }^{*}++\right.$ bufferptr $\left.!={ }^{\prime \prime \prime}\right)$;

while $\left(\left(\mathrm{c}={ }^{*}++\right.\right.$ bufferptr $\left.) !={ }^{\prime \prime \prime}\right)$

${ }^{*}(\mathrm{p}++)=\mathrm{c}$;

while $\left(\left(\mathrm{c}==^{*}++\right.\right.$ bufferptr $)==$, ' ||$c==' \backslash \mathbf{r}^{\prime} \| \mathrm{c}==$ 
${ }^{*} \mathrm{p}={ }^{\prime} \backslash 0^{\prime} ;$

finish = atof (valuestr);

if (finish > L)

finish $=\mathrm{L} ; /^{*}$ we do not want to go beyond the pile's length */

$/^{*} \quad$ Following Pile Driving Formula is based on Engineering News formula. The calculations for soil resistance and other parameters are applicable to single-acting steam hammer.

${ }^{*}$ Definitions

$R(i)=$ safe load on a pile, $l b$

$S(i)=$ average penetration in in/blow

$/^{*} \quad$ skin friction Resistance is computed iterate and cumulatively whereas end bearing is computed discretely

for $($ depth $=$ start; depth $<=$ finish; depth $+=$ delta_d)

\{

AcSf $=$ AcSf + SF (depth, Soil_Type, NSOILS, soil);

$\mathrm{Ri}=(\mathrm{SA} * 12) * \mathrm{AcSf} ;$

$\mathrm{Si}=\mathrm{HF}(\mathrm{E}, \mathrm{Ri}$, Hammer_Type, NHAMMS, hammer);

n blows $=$ n_blows $+\left(12^{-} / \mathrm{Si}\right)$

$\overline{\mathrm{Ti}}=(\operatorname{delta} \overline{\mathrm{d}} * 12) /\left(\mathrm{Si}^{*} \mathrm{~F}\right)$;

Variable Time $+=$ Ti;

fprintf (f), "\%6.0f \%10s $\% 10.2 \mathrm{f} \quad \% 12.4 \mathrm{f} \quad \% 8.2 \mathrm{f} \quad \% 8.2 \mathrm{f} \quad \% 8.2 \mathrm{f} \% 6.2 \mathrm{f} \backslash \mathrm{n}$,

depth, Soil_Type, Ri, Si, Ti, Variable_Time, AcSf, n_blows);

if (scr_out $==$ ' $y^{\prime} \|$ scr_out $\left.=={ }^{\prime} \mathrm{Y}^{\prime}\right)^{-}$

printf ("\%6.0f \%10s \%10.2f \%12.4f $\% 8.2 \mathrm{f}$

$\mathrm{Si}, \mathrm{Ti}$,

Variable_Time);

$\% 8.2 f \backslash n "$, depth, Soil_Type, Ri,

$/^{*} \quad$ Set a flag to test if the maximum allowable number of hammer blows per inch, 8 to 12 blows/inch as stated in Peurifoy (1970) and Canadian Foundation Manual (1978), has been exceeded or not. If hammer blow rate (inversely proportional to penetration rate) is more than that, then there is a chance the pile could be overstressed or damaged due to either 1) existence of an obstacle, 2) higher soil resistance, 3) Lower Hammer efficiency. So, lower bound for penetration rate $(\mathrm{Si})$ can be translated as $1 / 12(0.083)$ inch per blow $(1 / \mathrm{Si})$, or as Allowable Maximum Ti in minutes per $\mathrm{ft}$. The first one was chosen, and therefore, if that limit is reached at a given driving depth, the program further assigns a Boolean variable of the technical feasibility state to "False". The program returns the value of technical feasibility and the depth where stopped if it did not reach the refusal depth. CMSA will interpret these results and suggest a remedy action.

This program makes no allowance for obstruction */

if $(\mathrm{Si}<=0.083)\{$

printf ("Driving stopped at depth $=\% 4.0 \mathrm{f}$ since penetration

rate $\backslash \mathbf{n}^{\prime \prime}$, depth);

printf ("has reached its limit of 12 blows per inch. $\left(\mathrm{n}^{\prime)}\right.$;

Listing B.1 Drive.c Routine for Pile Driving (continued) 


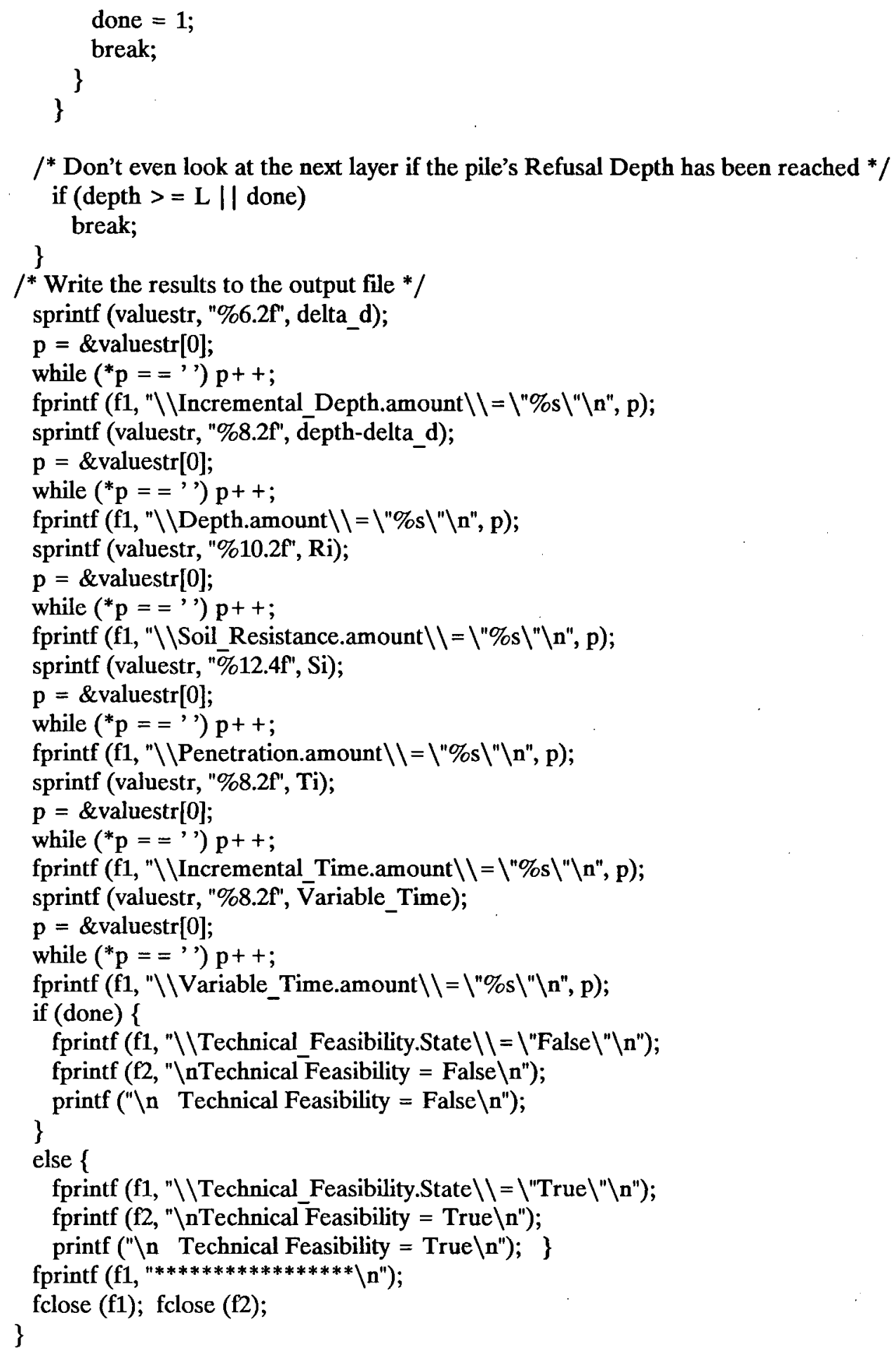

Listing B.1 Drive.c Routine for Pile Driving (continued) 


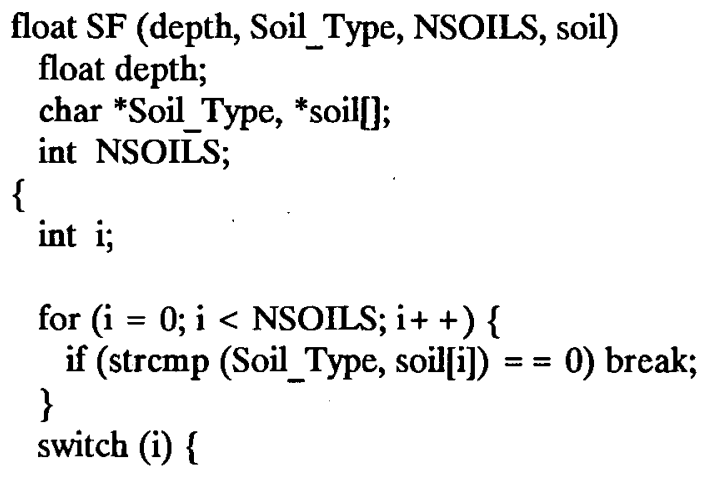

$/^{*} \quad$ Following step function equations were used for estimating skin friction. Those were derived, by interpolation, from Peurifoy (1970), table 17-4, pp. 483. They will be used as upper bound for the theoretical calculated skin friction */

case $0: /^{*}$ Soil Type 1 = Loose Sand */

if (depth $>0.0 \& \&$ depth $<=20.0)$ return $(17.5 *$ depth);

else if (depth $>20.0 \& \&$ depth $<=60.0)$ return $(1.88 *$ depth +312.50$)$

else if (depth $>60.0$ \&\& depth $<=100.0$ ) return $(1.88 *$ depth +312.50$)$

else if (depth $>100.0)$ return (500);

break;

case 1: $/{ }^{*}$ Soil Type 2 = Dense_Sand */

if (depth $>0.0 \& \&$ depth $<=-20.0$ ) return $(30.00 *$ depth);

else if (depth $>20.0 \& \&$ depth $<=60.0$ ) return $\left(2.50^{*}\right.$ depth +550.00$)$;

else if (depth > 60.0) return (700);

break;

case $2: / *$ Soil Type $3=$ Soft_Clay $* /$

if (depth $>0.0 \& \&$ depth $<=20.0$ ) return $(7.5 *$ depth);

else if (depth $>20.0 \& \&$ depth $<=60.0$ ) return $(1.25 *$ depth +255.00$)$;

else if (depth $>60.0 \& \&$ depth $<=100.0$ ) return $(1.25 *$ depth +255$)$;

else if (depth > 100.0) return (350);

break; 


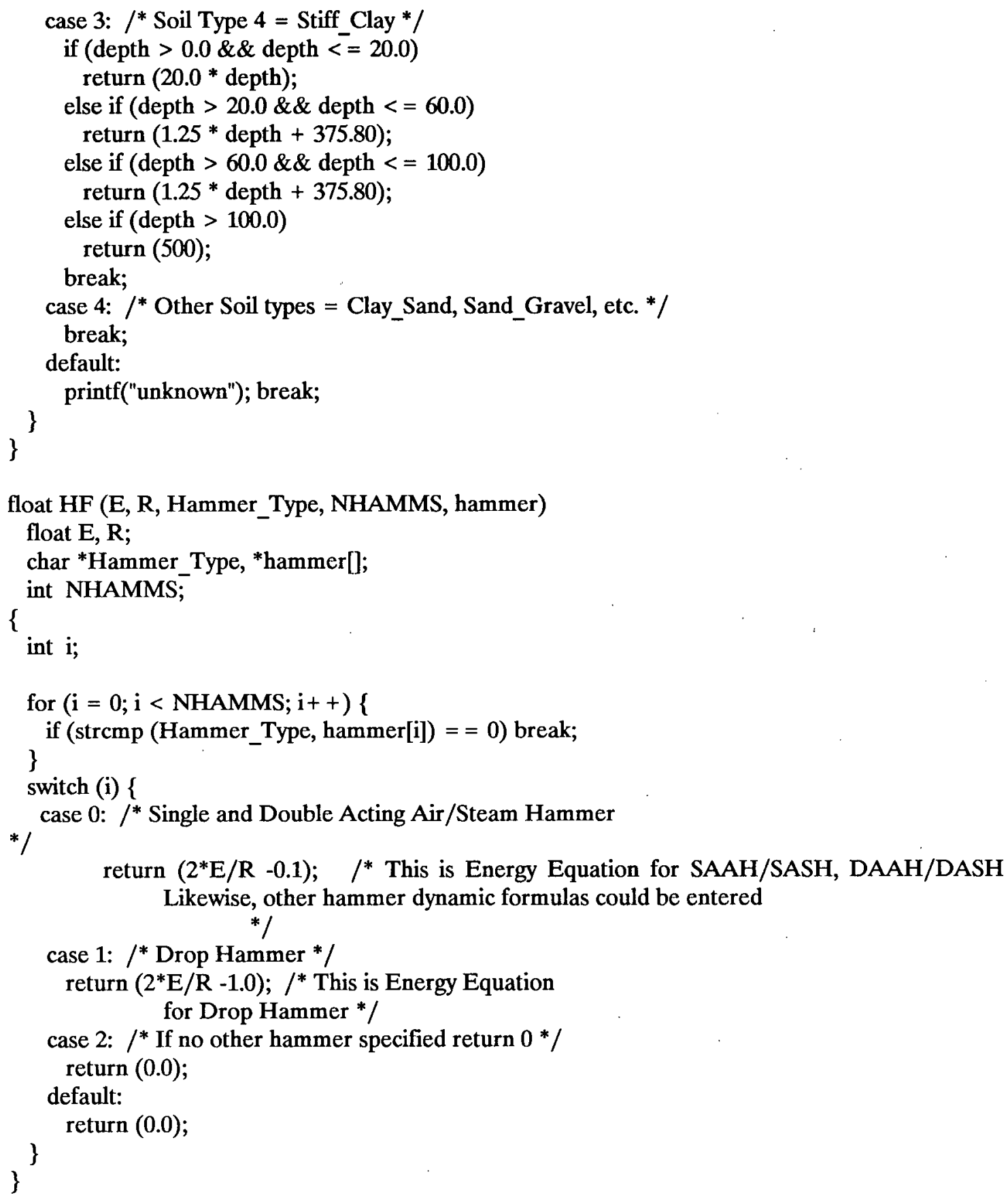

Listing B.1 Drive.c Routine for Pile Driving (continued) 


\section{Appendix C}

\section{Interviews}

\section{C.1 Introduction}

This appendix covers interviews conducted with some shoring speciality contractors. A survey was also designed to solicit input from a large number of contractors. It was not formally distributed and is therefore not included.

Both formal and informal meetings were held with specialty contractors on the topic of shoring methods including visiting a site where steel sheet pile driving activity was in progress.

\section{C.2 Minutes of Meeting with Dillingham Contractors}

Present: Alan Russell (ADR), Ibrahim Al-Hammad (IA), Stuart Brown (Chief Engineer, Dillingham)

Date: January 30th, 1990

Background: The meeting took place at Dillingham headquarters office on Wednesday morning, 9:45-11:45, on January 30, 1990 with the above participants. stuart Brown was the expert while ADR and Ibrahim acted as knowledge engineers. Mr. Brown is a senior engineer at Dillingham Contractors company. He has been involved in estimating, bidding and executing shoring projects. Discussion started after ADR briefed Brown about the methods selection problem and its relevance to Ibrahim's research work. Brown was given a outline of the proposed interview that contained an introduction to the subject matter, objectives of the interview for knowledge acquisition, and a brief tutorial about expert systems.

The discussion that ensued is summarized below.

Question 1:

Given there are two shoring alternatives steel sheet piles (SSP) versus soldier piles and lagging (SPL), what factors decide the selection of one at a high level without going into detailed analysis? 


\section{Answer:}

First, Brown indicated that SSP and SPL are similar in being positive systems: i.e. they contain water flow, and are safer to work with, and have a lower risk premium. Furthermore, ground conditions and local experience are the major considerations for shoring selection. Subsurface conditions in Vancouver are either good ground or "garbage".

Brown emphasized that expertise and availability of the equipment in the region or local area are major considerations in selecting a shoring type. For example, in the vancouver area, shotcrete with anchors is the prevailing method (with some risk) whereas in Toronto, it is soldier piles and lagging. Shotcrete in Vancouver has been used for the last 15 to 20 years because of the good soil, it is 3 times faster than soldier piles and lagging, and it is more economical. It was also emphasized that standard local practice is very important when selecting a shoring method.

In Vancouver, common shoring methods are shotcrete, soldier piles and lagging, and other soil nailing; steel sheet piles are typically used for cellular and marine cofferdams.

Two major criteria were identified for evaluating alternatives: price, and local risk assessment. Those are quantified in terms of monetary value with expected probabilities. The bottom line is to use the most economical system.

Question 2:

Does the excavation activity have a major influence on shoring selection?

\section{Answer:}

Shoring activity is more expensive than excavation. Excavation is insensitive to shoring method and is only a secondary consideration. As for ease of excavation, it is preferable to use anchors, not struts. Using struts causes an extra cost of approximately $30 \%$ by requiring material handling equipment (mainly cranes).

Question 3:

How does the firm makes decisions about selecting a shoring method?

\section{Answer:}

A brainstorming session takes place to discuss alternatives globally, with the decision being based mainly on cost and confidence that the method will work. A contingency allowance is part of the decision making. of paramount 
importance are historical records of productivity for different methods in making the choice.

For instance, for one of the projects they bid, the soil condition was of sand and gravel with high water-table for a 30 foot deep cut. Two alternatives were considered:

1. Shotcrete which requires an open face in order to be sprayed to a pre-designed thickness. Local risk is of wall collapse and how big the open face is. If there is a washout failure, then, the resulting loss includes the costs of crews not working, crews fixing the problem, and the loss of equipment. The risks and other potential losses are assessed using educated guesses by going through what if questions.

2. Soldier piles and Lagging which is a positive system.

At the end, having considering all the factors, shotcrete was selected.

Question 4 :

How much detailed design is performed before an alternative is pruned? For instance, consider SSP and SPL.

\section{Answer :}

First, the level of design depends on the likely alternatives, the size of the project, profit, number and identity of competitors, etc. For SSP and SPL, a minimum preliminary design that takes approximately two hours includes sizing the materials, spacing and sizing of the supports and pricing out the materials.

other considerations include purchase of materials and current material inventory. An attempt is always made to use materials on hand as opposed to buying new materials.

sizing is done for H-piles and steel sheet piles, struts, wales, and anchors.

Question 5:

What are the standard lengths of SSP used in the field and how important is it to optimize their length for more efficient driving ?

Answer:

That depends upon site location, site layout (which influences the maximum length that can be welded on site), means of transportation (determines if large sizes are 
possible), manufacturers (typically they come in $30^{\prime}$ and 60" lengths, and sometimes up to $120^{\prime}$ ), depth of the cut, size of crane and hammer for pile driving.

When steel sheet piling is available from other projects, short lengths will be used, even though additional welding is required, because of the economies involved. The biggest advantage of welding piles before driving them, is to have minimum hammer setting time during which the crane is idle. Typically, this is 10-15 minutes, whereas the variable time takes $1 / 4$ to $1 / 3$ of total driving time (e.g. 5 minutes for 100 foot using single Acting Air Hammer (SAAH) in loose sand).

Follow-up question:

If time is a major concern and a steel sheet piling system was specified by the design engineer, does shortening the project duration alter the SSP design and are other shoring methods evaluated as well?

\section{Answer:}

Well, you might use heavier sheets with a retaining system of anchors, if possible. This is more expensive than using lighter sheets. However, there is less risk of sheets being overstressed or damaged, resulting in delays.

Question 6:

When time is an issue, what shoring method is preferable?

\section{ANSWER:}

Sometimes, time can be a very important factor. For instance, for shotcrete, it is fast in wide open excavation whereas narrow cut may require a staged excavation (or vice versa). For a street cut project, Dillingham was asked to close the street for a week and use decks over the cut. For such a case, soldier piles and lagging are preferable since this system provides a more rigid support for passing cars and trucks, although it is less economical. The social impact of not disturbing traffic had more priority and overruled shoring economics. For shotcrete, the site is left open in stages, and you have a short time to work due to short soil stand up time for spraying and anchor installation. other follow-up crews have more time to work.

In order to speed up construction duration, one may consider:

1. Using fast setting material;

2. double shifting; and

3 . overtime. 
Remember for SPL, a staged installation approach is required, similar to shotcrete (excavation then lagging). Shotcrete needs sufficient length -- a minimum of a 300 foot long cut. Tunnel dimensions have an influence on selecting methods. For instance, a narrow tunnel follows a linear process whereas a wide tunnel allows more than one crew in one space compartment unit. Thus for a narrow tunnel, SSP is more preferred than SPL due to difficulty of installing lagging, which makes it more difficult to excavate.

Question 7 :

How accurate are the estimates when alternatives are being pruned ?

\section{Answer:}

Normally, for preliminary design, cost estimates are within $10 \%$ of the detailed design estimate,

Question 8:

How do ground conditions influence the risk for shoring?

\section{Answer:}

If encountered ground conditions are worse than anticipates then use large SSP and big struts. This may increase the cost, but it shortens time and is safer (less risk). For instance, SSP is much faster in uniform loose soil where there is no obstruction or tough driving.

\section{Question 9 :}

How do you prune alternatives based on technical feasibility without considering risk or under the same risk?

\section{Answer:}

For soft soils, i.e., loose sands and silts with high water level, soil containment is important. You can not afford to lower the water table, so the choice becomes ssP. If the soil is till then SSP is not feasible due to hard driving.

For loose sand and gravel, use H-piles. A problem with soldier piles and lagging is that to install the lagging, you need an open face; leaking water may cause a problem.

H-Piles are specified and spaced to support soil arching, whereas, lagging is used to contain water and soil. You may use pre-drilled $\mathrm{H}$-Piles to reduce the chance of damage. For medium dense sand and gravel, both SSP and SPL can be used. For till and sand, it is not economical to use SSP.

For Cofferdam, heavy SSP sections are used to minimize the number of supports; anchors are preferred because they are 
cheaper than struts and make construction faster. If the ground is loose, or swampy, then anchors can't be used. Anchors should be used whenever soil easement, and property access permission conditions are realized. A disadvantage of using struts is that it ties up a crane.

Question 10: What hammers are preferred for SSP driving?

\section{Answer:}

First, SSP is rarely used in Vancouver since soil is either good (strong ground) or "garbage". If SSP is used, vibratory hammers are the most efficient. The speed of driving is a function of the length of SSP, weight of the SSP unit, and vibratory hammer size. When choosing a vibratory hammer, the rule of thumb used is to select the one with the highest energy.

Keep in mind that set up time is 3 to 4 times the driving duration. Use a greedy approach to select the vibratory hammer size. Using the biggest one without overstressing the pile results in putting contingency up front in case of difficult driving. There will be no need to change the hammer, thus avoiding risks if difficult conditions are encountered.

Single Acting Air Hammers (SAAH), Double Acting Air Hammers (DAAH), and Drop hammers are used for soft driving. Air hammers (every blow 8 inches to 2 foot) requires adjusting the crane. If diesel hammer is used, there has to be enough soil resistance for pile driving, i.e., it is more suitable for hard material which exhibits higher soil resistance than those of the air hammers.

Normally, two piles are driven together, however, for tough driving drive one pile at time. Note that hammer vertical mobility and attachment are important. In essence, you do not want the pile to penetrate too much per blow such that it slips out of the leads and thus requires set up time for each blow.

Question 11:

If, for example, a diesel hammer has been recommended, what model should be selected?

\section{Answer:}

It depends on the length and weight of the pile and other factors. Rules of thumb to use are:

1. The weight of the hammer ram should be $1 / 3$ of pile weight or more; and

2. Piles could take up to 12 blows per inch. 
$\mathrm{H}$-piles come in 40 to 60 foot lengths. Thus, many suboptimal situations could be contemplated by trade-offs among pile length and weight, cost of material and equipment and construction efficiency.

SSP comes in different sizes lengths (depends on mode of transportation) from $20^{\prime}$ to $70^{\prime}$. The goal is to minimize welding.

Question 12:

If you have two layers of soil, for example, the first one is loose soil and the second is dense soil, how do you select a hammer?

\section{Answer:}

Start with a Drop/Air hammer for the first layer, then use diesel hammer for the second layer. Use a vibratory hammer for loose to medium dense silt and sand.

Question 13:

What is the average setup time and driving time for pile driving? our algorithm predicts $31 / 2$ minutes for driving time only for a Double-Acting Air Hammer for loose sand and 100 feet driving depth?

\section{Answer:}

If it is really cooking, it takes 3 to $31 / 2$ minutes for pile setup time, and it takes 15 to 20 minutes for driving the pile to the refusal depth if uninterrupted (i.e. total variable time). For the latter, in one instance, the variable time duration was 5 minutes for 100 deep excavation when single acting air hammer was used to drive a steel sheet pile in loose sand.

At the end of the interview, Brown drew a number of useful articles for Pile Buck magazine to our attention. 
Rules Deduced from this Interview: Two major factors are considered in evaluating shoring alternatives: price and risk assessment. Both are quantified in terms of monetary value. The following rules are a first attempt at encoding some of Mr. Brown's experience. These, rules will be compared to what is available in the literature in an attempt to adapt them to CMSA prototype when appropriate.

Those rules are written in IF THEN format as crude rules of thumb, shown in listing C.1 below.

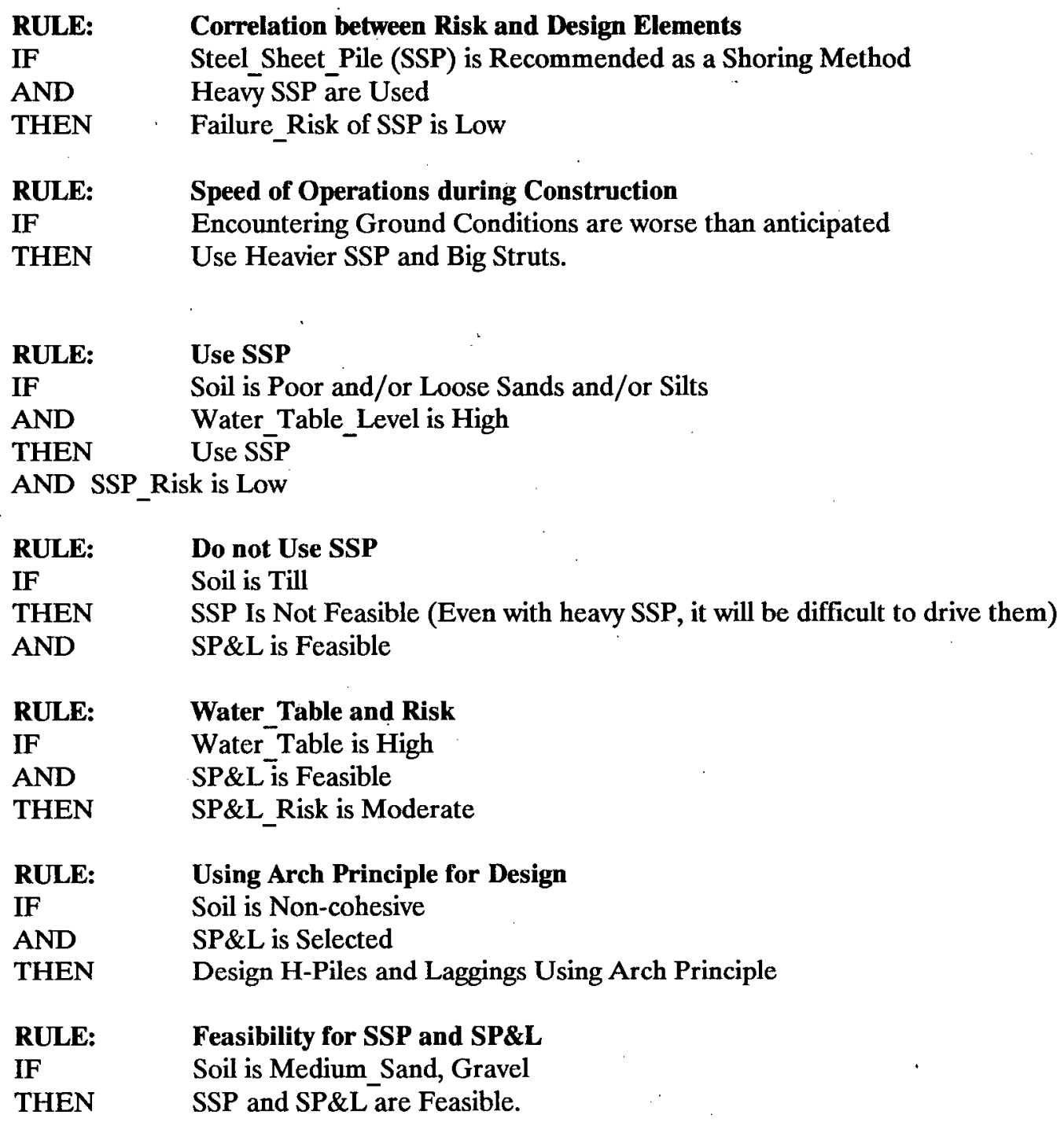




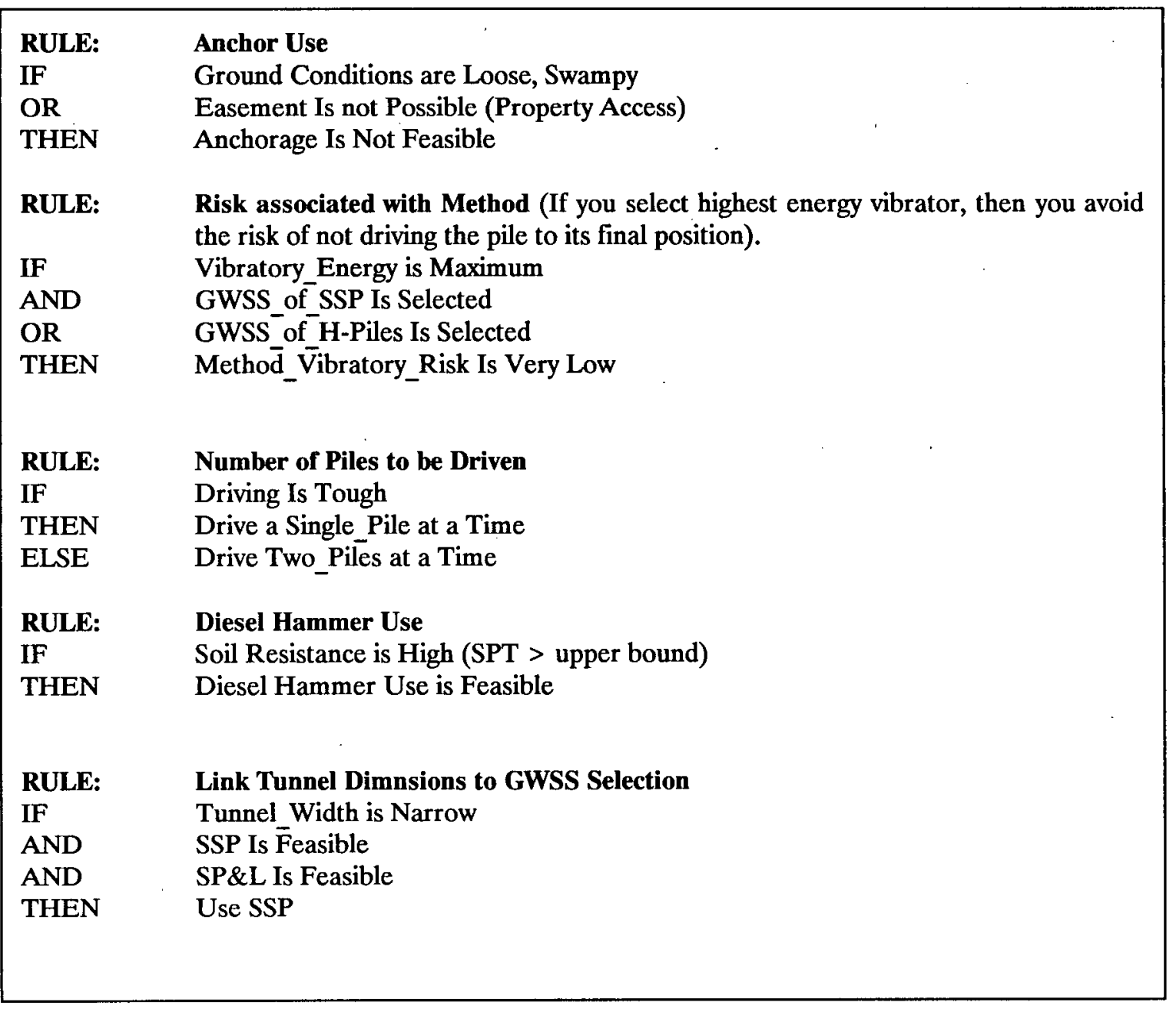

Listing C.1 Extracted Rules of Thumb (continued) 


\section{C.3 Minutes of Meeting with Quadra Construction}

Present: John Simonett, President, Quadra Construction, and Ibrahim Al-Hammad

Date: March 24th, 1990

Background: Quadra is a small size company that has little documented experience in selecting hammers. It is experienced in the driving of a wide range of pile types.

First, I introduced the subject matter of this visit by briefing John on expert systems technology and the problem of method selection. Furthermore, the importance of vibratory hammer selection was stressed (that was one of the main purposes of this meeting since a logic procedure had already been developed for Impact Hammers).

The purpose of the meeting was several-fold:

1. To examine and compare the logic of the prototype regarding steel sheet piling sizing and selection with the driving equipment required (hammers and cranes). It was found that such the logic developed to date for the prototype CMSA was similar to what is practiced by this firm, which helped confirm the validity of our approach.

2. An algorithm has already been developed to predict the productivity of impact hammers based on energy equations. However, sheet piling is most effectively driven, when conditions allow, by vibratory pile drivers (in favorable conditions, driving by vibratory is 10 times faster than driving by impact hammer). So far, there is nothing in the literature that relates vibratory pile driver productivity to its type or energy nor to the soil type. Thus, I wanted to know how a vibratory pile driver is selected. The answer was in the form of a rule of thumb -- select a vibratory driver with the highest horse-power. Typically, a vibratory pile driver drives steel sheet piles $20 \mathrm{ft} / \mathrm{min}$ in good conditions whereas it drives them $2 \mathrm{ft} / \mathrm{min}$ in bad conditions.

I presented John with an article from Pile Buck in which a vibratory driver is sized based on pile weight vs. soil blow count (STP) charts to get vibratory dynamic force. John was intrigued and tried to verify the charts with a pre-selected ICE 216 vibratory hammer employed at a job site. However, there was no agreement in the results. John has commented 
that this case study does not fall within the range of the charts and perhaps may resemble the worst condition.

Subsequent discussions yielded the following information:

1. Cost breakdown for sheet piling driving revealed that the cost of material is approximately $3 / 4$ of total cost whereas $1 / 4$ is labor cost and equipment (the labor cost is almost equal to the equipment cost). Some representative costs are shown below in Table C.1.

1. Vibrator Model

Model ICE 812

Model ICE 216

2. Crane

35 Ton

70 Ton

150 Ton

3. Crew

4 men / day
Dynamic Force

(Tons)

145.5

36.4
Rent

$\$ 11000 /$ month $\$ 7000 /$ month

Rent

$\$ 6500 /$ month $\$ 8500 /$ month $\$ 12000 /$ month

Cost $\$ 1100 /$ day

Table C.1 Sample Pile Driving Resources Unit cost [Quadra 1990]:

In comparison with other shoring methods, material costs and savings are of major concern for SSP rather than labor or equipment.

2. Pile driving time is 3 to 4 times the set up time.

3. Length of pile is constrained by transportation means and the crane boom availability.

4. Pile driving is more efficient if driven by pairs -- in fact sheets have been ordered in doubles from the factory, or welded at the site.

5. A vibratory hammer typically yields a maximum rate of production of $20 \mathrm{ft} / \mathrm{minute}$ under good conditions to a minimum of $2 \mathrm{ft} / \mathrm{min}$.

6. If the soil is cohesive or very compacted, then a vibratory pile driver is not suitable.

7. Hammer rental cost does not vary linearly with horsepower. 
8. Crane capacity and configuration must suit pile dimensions and the hammer weight. Normally, crane cost is equal to the hammer cost.

9. Vibratory hammers are more productive for nondisplacement piles, however, they are more expensive to rent and mobilize.

10. Hydraulic hammers are more productive due to their higher frequency for the same energy. There is no local experience with them, and apparently, they are more expensive.

11. Risk assessment was mentioned as important. However, the discussion was not elaborated enough to understand the decision process nor how could it be linked to methods selection.

At the end, I asked Mr. Simonett if I could visit some of their nearby pile driving projects. A month later, $M r$ Simonett informed me about a project they were involved in. A description of the site visit follows.

\section{C.4 Project Site Visit}

Date: April 28, 1990

A project involving Quadra included driving heavy sheet piles for a caisson. From a top view, it consist of a rectangular shape 22 feet wide by 43 foot long with 50 foot long sheet piles and a 216 ICE Vibrator (Low Frequency). At first, an interior frame is set up to hold the piles together at the ground level. It is then lifted by a crane to a height of $25 \mathrm{ft}$ and seated on $4 \mathrm{H}-\mathrm{Piles}$. SSP piles are first seated by their own weight to 1 to 2 foot, then they are inter-connected with their adjacent piles. The foreman explained that he designates some piles as key piles which are welded to the frame- at each cycle welding took place at mid-level frame (at 20 ft elevation) and at ground floor. Piles were driven in vertical rounds and in pairs. First round, vertical driving is $15 \mathrm{ft}$; second round, it drops to $11 \mathrm{ft}$ due to more resistance, and to $8 \mathrm{ft}$ and so forth. Furthermore, if piles are hard to drive then they are driven in singles. The foreman mentioned that oiled sheet piles provide less resistance between adjacent piles and that new ones are easier to drive and extract than old ones due to rust.

Description of A sheet Pile Driving Cycle: Sequential pile driving proceeds in waves around the cofferdam. When driving reaches the key sheets, a worker has to un-weld them from the top and bottom frames. This causes some delay 
during which the crane operator, laborer, and vibratory operator are idle.

It was observed that a fair amount of the laborers time was consumed in aligning the vibratory pile driver in position over the piles and in moving the pile driver cables around. 


\section{Appendix D}

\section{Knowledge Base Sample, Interviews, Unit Costs, and Sample Data Base}

\section{D.1 Introduction}

This appendix contains listings for:

1. A sample CMSA knowledge base using NExpert object syntax, printed as a text file. This is a partial CMSA that excludes the Risk component.

2. Vibratory hammers selection knowledge.

3. Unit costs for materials and crews used for CMSA quantity take-off and cost estimate calculations.

4. A sample of structural members for ground wall support system (GWSS), and data bases used in CMSA prototype. 


\section{D.2 Partial Listing of CMSA Knowledge Base}

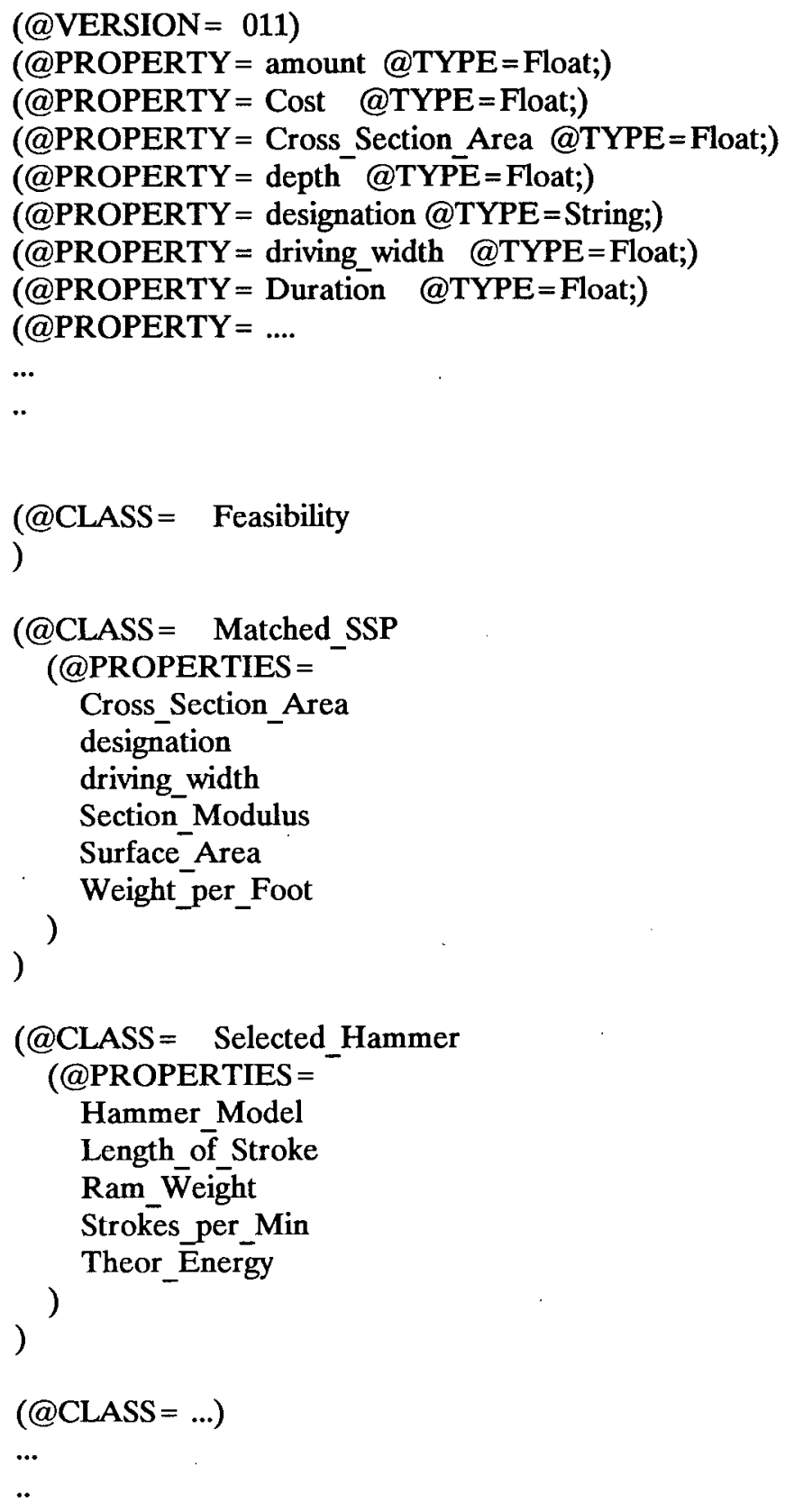




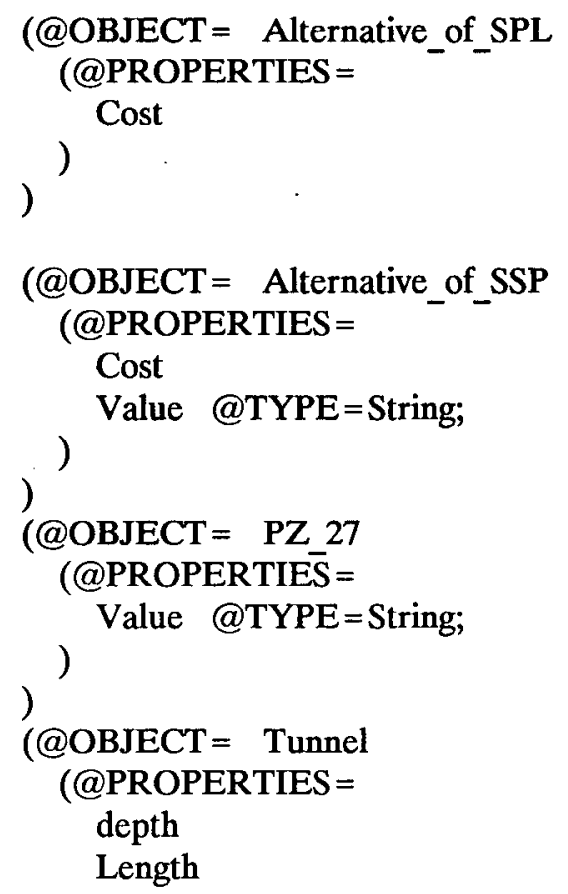
selection (section modulus is measured in in 3 ). 15 foot represents vertical spacing between the struts";@WHY="This rule finds the section modulus for a steel sheet pile in order to select a suitable section.";

(@LHS = (Is (Soil.type) ("Loose_Sand"))

Listing D.1 Partial Listing of CMSA (continued) 


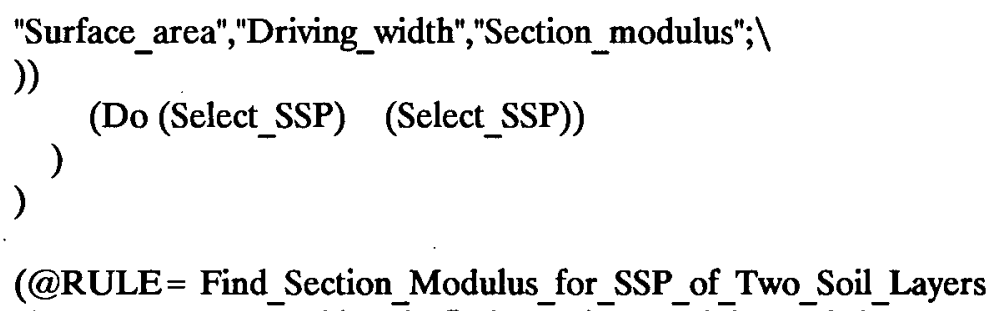
synthesized method under the soil/hammer/pile is not technically feasible then control resets the pile driving pattern status into In_Singles.

The technical feasibility status will be checked again. If it is "True", then the latter driving strategy is adopted. If the technical feasibility state is "False", then altering hammer/pile selection is advised. Such advise is not yet incorporated in CMSA).

\section{Listing D.1 Partial Listing of CMSA (continued)}




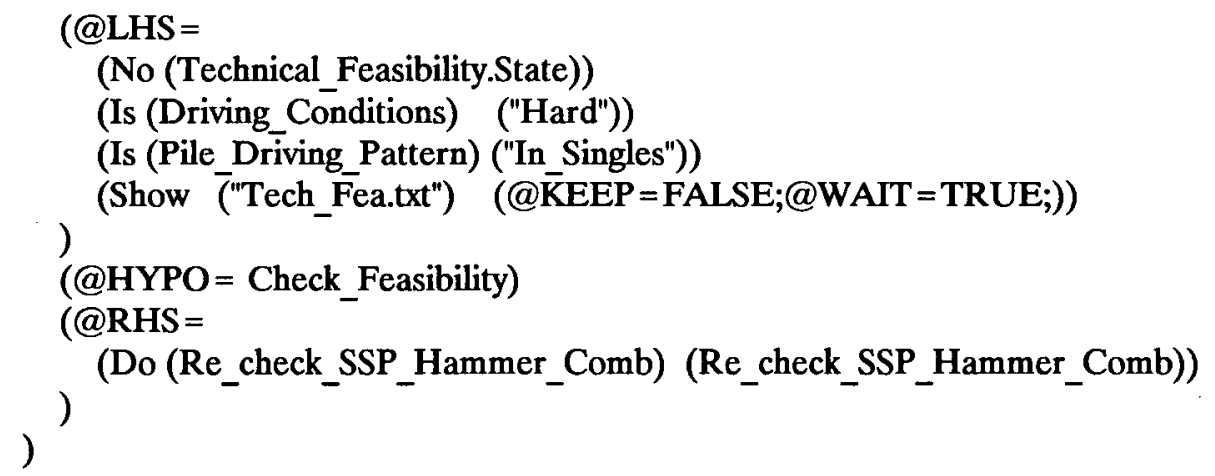

Listing D.1 Partial Listing of CMSA (continued) 
(@RULE $=$ Technically_Feasible_Alternative

@INFCAT=3;

$@$ COMMENTS="If the driving conditions are soft, pile driving pattern is "In_Singles", and the method is not technical feasible, then compute the sheet pile production rate.";

(@LHS=

(Yes (Technical Feasibility.State))

(Is (Driving_Conditions) ("Soft"))

(Is (Pile_Driving_Pattern) ("In_Singles"))

)

(Show ("Tech_Fea.txt") (@KEEP=FALSE;@WAIT=TRUE;))

(@HYPO= Check_Feasibility)

(@RHS=

)

(Do (Compute_Production_of_SSP) (Compute_Production_of_SSP))

)

(@RULE = Technically_Feasible_Alternative

$@$ COMMENTS = "If the driving conditions are soft, pile driving pattern was "In_Pairs", and the method is technically feasible, then compute the sheet pile production rate.";

(@LHS =

(Yes (Technical Feasibility.State))

(Is (Driving_Conditions) ("Soft"))

(Is (Pile Driving Pattern) ("In Pairs"))

)

(Show ("Tech_Fea.txt") (@KEEP=FALSE;@WAIT=TRUE;))

(@HYPO= Check_Feasibility)

(@RHS= )

(Do (Compute_Production_of_SSP) (Compute_Production_of_SSP))

)

(@RULE $=$ Technically_Feasible_Alternative

$@$ INFCAT $=3$;

@COMMENTS="If the driving conditions are soft, pile driving pattern is "In_Pairs", and the method is not technically feasible, then reset the pile driving pattern state to "In_Singles" and re-fire the technical feasibility rule (This rule is analogous to a loop construct in a conventional programming).";

(@LHS=

(No (Technical_Feasibility.State))

(Is (Driving_Conditions) ("Soft"))

(Is (Pile_Driving_Pattern) ("In_Pairs"))

(Name "("In_Singles") (Pile_Driving_Pattern))

(Show ("Tech_Fea.txt")

Listing D.1 Partial Listing of CMSA (continued) 


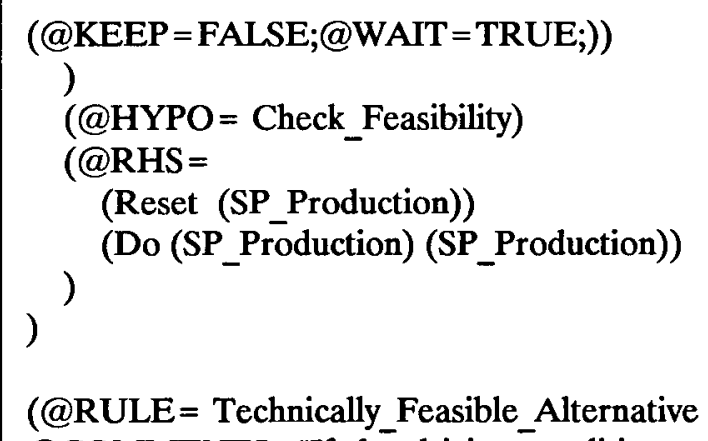
technical feasibility state is "False", then terminate the session. Furthermore, $\bar{a}$ text file may be displayed to announce that there is no feasible solution under the session scenario. The hypothesis (Re_check_SSP_Hammer_Comb) has not been implemented yet. Its future object is to recommend changes in hammer/pile designs to arrive at feasible method synthesis";

(@LHS=

(No (Technical Feasibility.State))

(Is (Driving_Conditions) ("Soft"))

(Is (Pile_Driving_Pattern) ("In_Singles"))

)

(Show ("Tech_Fea.txt") (@KEEP=FALSE;@WAIT=TRUE;))

(@HYPO= Check_Feasibility)

(@RHS = )

(Do (Re_check_SSP_Hammer_Comb) (Re_check_SSP_Hammer_Comb))

)

(@RULE= Production_Measures_of_SSP_for_Driving_Single_Pile

$@$ COMMENTS $=$ "Total $\overline{1}$ driving time of $\overline{-}$ a set of piles to the refusal depth consists of two components; variable component which depends on soil/pile/hammer scenario, and fixed which is dependant on pile welding and positioning and movement of pile driver. A pile is driven in several segments, i.e., in a pile series to the refusal depth. For the variable time computation, it is assumed that there is no pile driving interruption; the fixed time component is equal to multiple of set up times

;@WHY = "This is to measure the productivity performance of sheet pile and a given pile driver.";

(@LHS=

(Is (Pile_Driving_Conditions) ("In_Singles"))

(Name (Fixed_Time_per_given_Hammer)

(Fixed

Time_per_pile.amount))

(Show ("P Length") (@KEEP=FALSE;@WAIT=TRUE;))

(Name (CEIL(Tunnel.depth/SSP.Standard_Length)*Fixed_Time_per_pile.amount)(

Fixed_Time_per_SSP_Unit_Width.amount))

(Name (CEIL(T) Tunnel.depth/SSP.Standard_Length)) (Number_of_Piles_per_SSP_ Unit_Width))

Listing D.1 Partial Listing of CMSA (continued) 


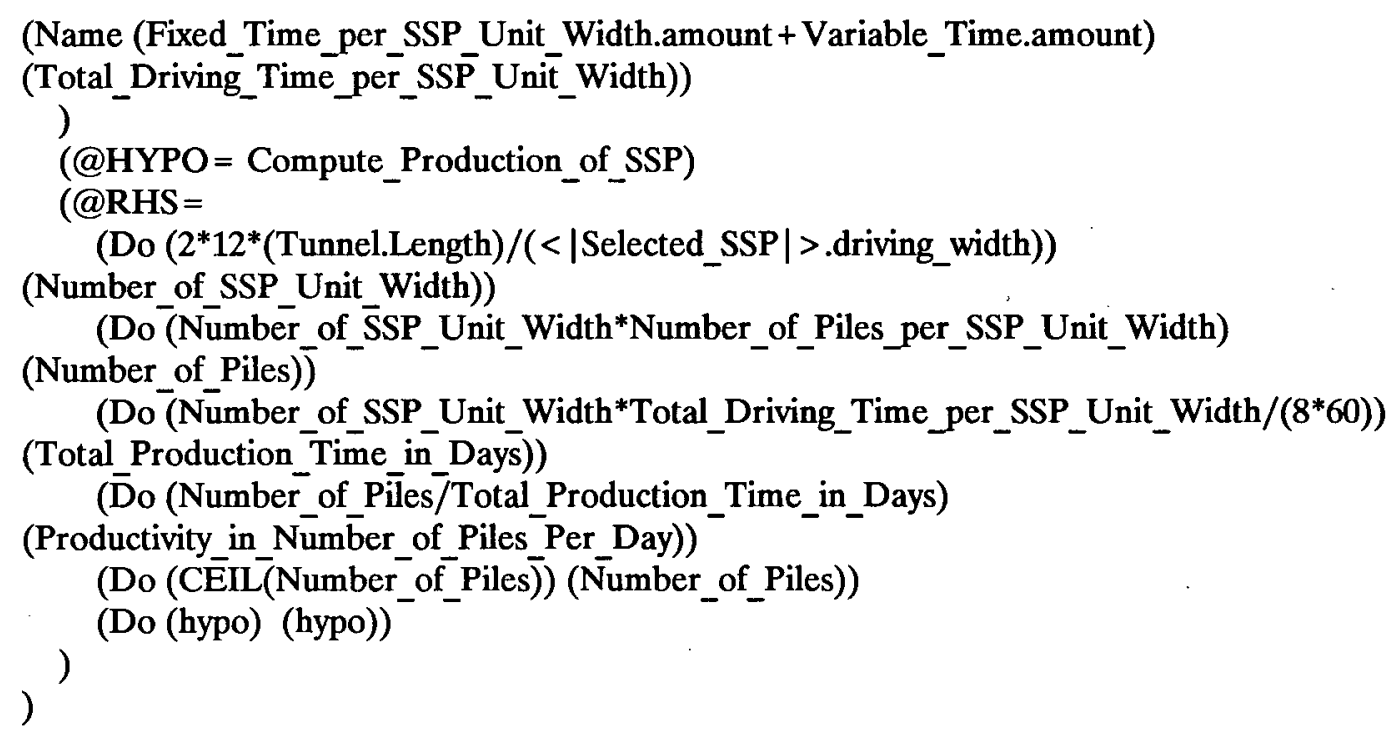

(@RULE $=$ Production_Measures_of_SSP for_Driving_Single_Pile

$@$ COMMENTS $=$ "Total time consists of two components: variable component which depends on soil/pile/hammer scenario, the fixed which is dependant on pile welding and positioning, and movement of pile driver. Theoretically the number of sheets used is the same, however, the delivery could be "In-Pairs" requiring less fixed time for the crane. The computation of the total driving time is adjusted by identifying Pile_Driving_Conditions state (In_Pairs, In_Singles). For the former, driving sheets in pairs, the total driving variable time is perhaps less than the latter, however, the total driving fixed time (as number of the setups) is less than the former.

The "P Length" text file explains how pile segment length effects the total driving time; $@ W H \bar{Y}=$ "This is to measure the productivity performance of sheet pile and a given pile driver.";

(@LHS=

(Is (Pile_Driving_Conditions) ("In_Pairs"))

(Name (Fixed_Time_per_given_Hämmer) (Fixed_Time_per_Pair_of_piles.amount)

)

(Show（"P_Length") (@KEEP=FALSE;@WAIT=TRUE;))

(Name

(CEIL(Tunnel.depth/SSP.Standard_Length)*Fixed_Time_per_Pairs_of_piles.amount)

(Fixed_Time_per_Pair_of_SSP_Unit_Width.amount)

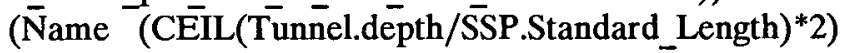

(Number_of_Piles_per_Pair_of_SSP_Unit_Width)

(Name ${ }^{-}$(Fixed_Time_per_Pair_of_SSP_Unit_Width.amount + Variable_Time.amount) (

Total_Driving_Time_per_Pair_of_SSP_Unit_Width))

)

(@HYPO=Compute_Production_of_SSP)

(@RHS=

(Do $(2 * 12 *$ (Tunnel.Length) $/(<\mid$ Selected_SSP $\mid>$.driving_width))

(Number_of_Pair_of_SSP_Unit_Width))

Listing D.1 Partial Listing of CMSA (continued) 
(Do (Number_of_Pair_of_SSP_Unit_Width*Number_of_Piles_per_Pair_ of_SSP_Unit_Width) (Number_of_Pairs_of_Piles))

(Dō (Nümber_of_Pair_of_SSP__Unit_Width*Total_Driving_Time_per_

Pair_of_SSP_Unit_Width $\left./\left(\bar{\delta}^{*} 60\right)\right)$ (Total_Production_Time_in_Days))

(Do $\left(2^{*}\right.$ Number_of_Pair_of_Piles/Total_Production_Time_in_Days)

(Productivity_in_Number_of of Piles_Per_Day))

(Do (CEIL_(Number_of_Pair_of_Piles)) (Number_of_Piles))

)

(Do (hypo) (hypo))

)

(@RULE = Write_Desired_Output_Results

(@LHS=

(Name (1) (a))

)

(@HYPO=hypo)

(@RHS=

(Write ("Results.nxp") (@TYPE=NXP;@FILL=NEW;@UNKNOWN=TRUE;@ATOMS= Technical_Feasibility, \Number_of_Piles,Total_Production_Time_in_Days, $\backslash$

Productivity_in_Number_of_Piles_Per_Day,Selected_Steel_Pile, $\backslash$

Pile_Driver, $\overline{\mathbf{H}}$ -

(Show ("Results.nxp") (@KEEP=FALSE;@WAIT=TRUE;))
((Do (Done) (Done))

)

)

(@RULE= Select_GWSS_of_SSP

(@LHS =

(Name (10) (a))

(Show ("Text1.txt") (@KEEP=FALSE;@WAIT=TRUE;))

)

(Is (GWSS) ("Steel_Sheet_Pile"))

(@HYPO= Select_A_GWSS)

(@RHS= )

(Do (Select_GWSS_of_SSP) (Select_GWSS_of_SSP))

)

(@RULE = Select_GWSS_of_SPL

(@LHS =

(Name (10) (a))

(Show ("Text1.txt") (@KEEP=FALSE;@WAIT=TRUE;))

)

(Is (GWSS) ("Soldier_Piles_and_Lagging"))

Listing D.1 Partial Listing of CMSA (continued) 


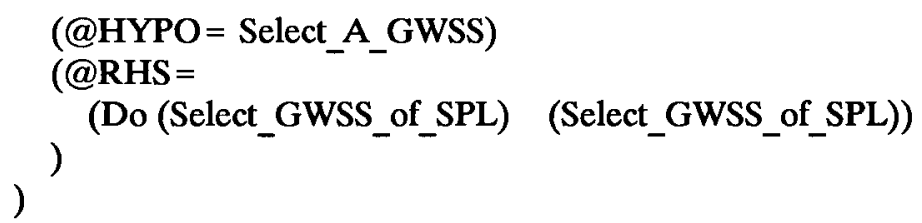

Listing D.1 Partial Listing of CMsA (continued) 


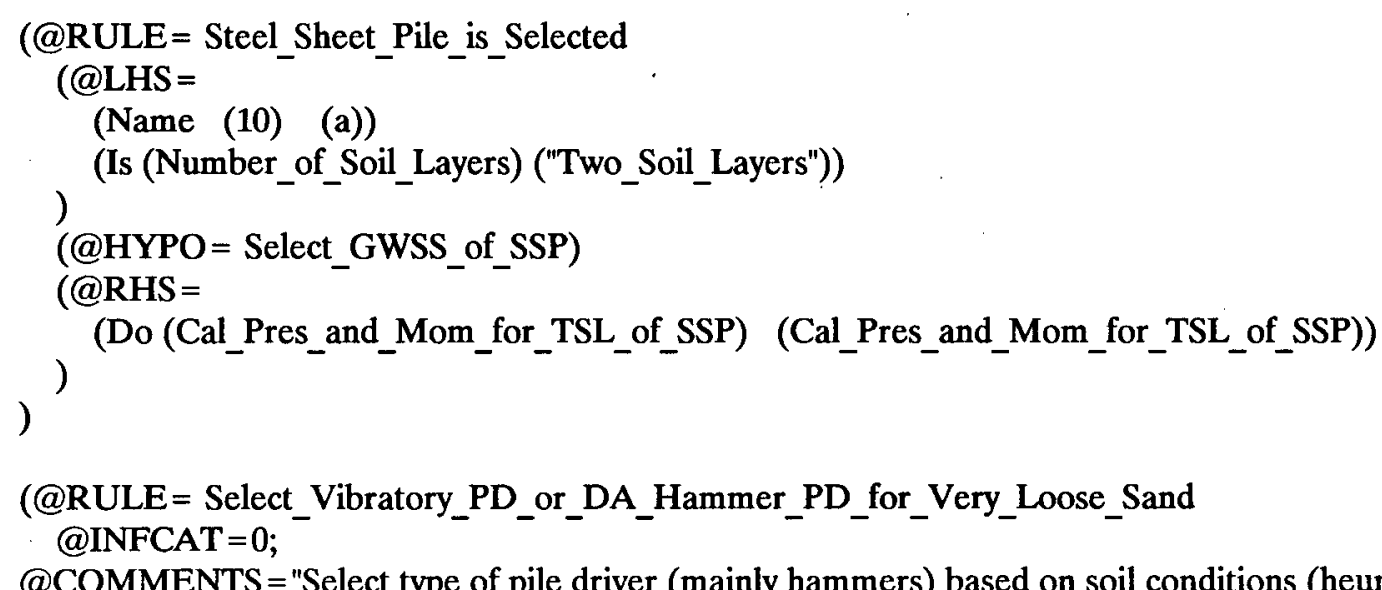

@COMMENTS = "Select type of pile driver (mainly hammers) based on soil conditions (heuristics found in Hunt 1979)";

(@LHS=

$$
\text { (@HYPO=Select_Pile_Driver) }
$$$$
\text { (Is (Soil.type) ("Very_Loose_Sand")) }
$$$$
\text { (@RHS= }
$$$$
\text { (Let (Pile_Driver) ("Vibratory")) }
$$

(@RULE= Select_Vibratory_PD_or_DA_Hammer_PD_for_Very_Loose_Sand $@ \mathrm{COMMENTS}^{-}=$"Select type of pile driver (mainly hammers) based on soil conditions (heuristics found on Hunt 1979)";

(@LHS= )

(Is (Soil.type) ("Very_Loose_Sand"))

$(@ \mathrm{HYPO}=$ Select_Pile_Driver $)$

(@RHS=

(Let (Pile_Driver) ("Double_Acting_Hammer"))

)

(Do (Ret_Hammer_Energy) (Ret__Hammer_Energy))

)

(@RULE $=$ Select_Vibratory_PD_or_DA_Hammer_PD_for_Very_Loose_Sand $@ \mathrm{INFCAT}=0$;

@COMMENTS="Select type of pile driver (mainly hammers) based on soil conditions (heuristics found in Hunt 1979)";@WHY="Inference category $($ INFCAT $=0$ ) is set to 0 in order to set a low priority for selecting a vibratory pile driver as opposed to impact hammers.";

(@LHS= 


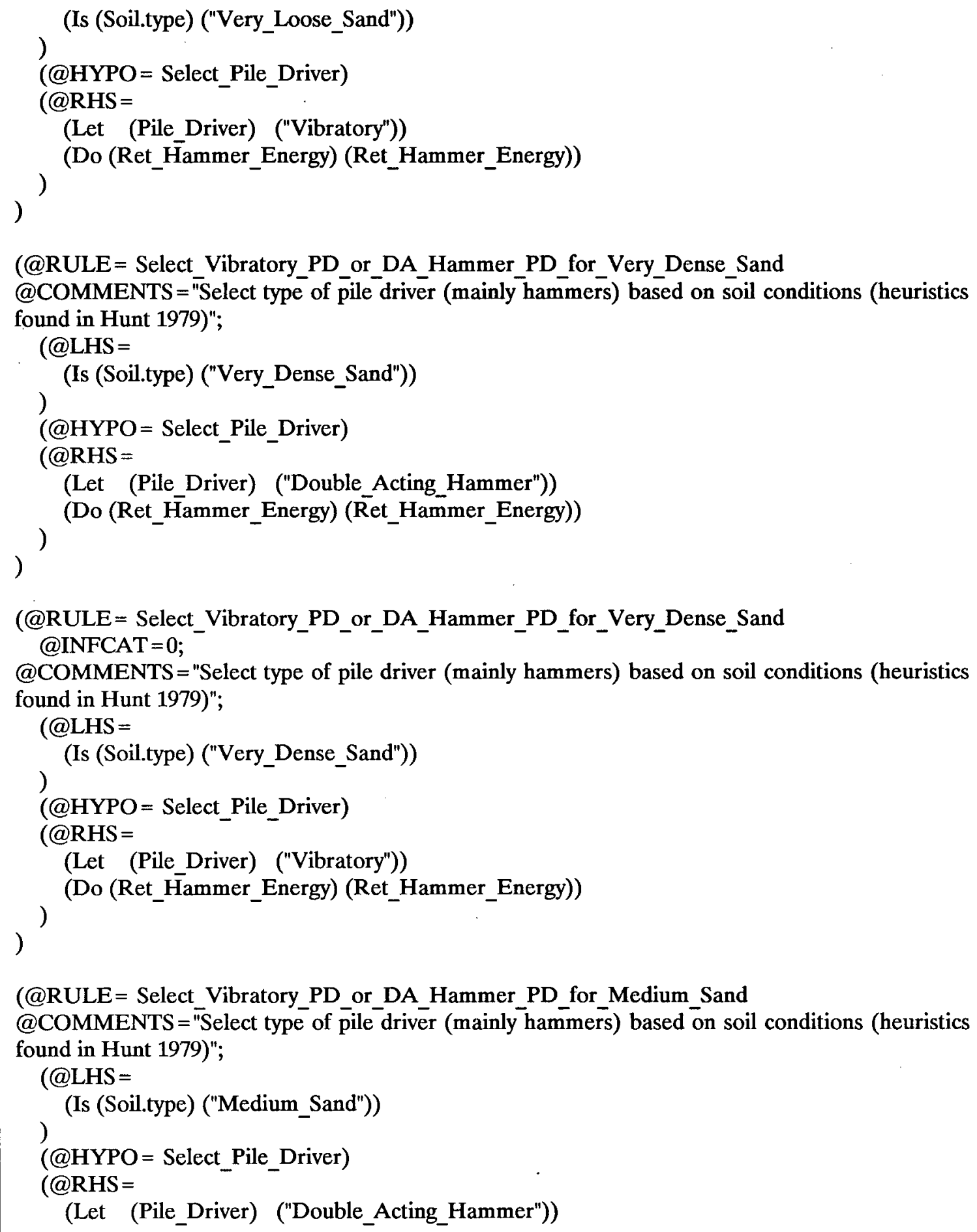

(@RULE = Select_Vibratory_PD_or_DA_Hammer_PD_for_Medium_Sand $@$ COMMENTS = "Select type of pile driver (mainly hammers) based on soil conditions (heuristics found in Hunt 1979)";

(@LHS = )

(Is (Soil.type) ("Medium_Sand"))

(@HYPO= Select_Pile_Driver)

(@RHS=

(Let (Pile_Driver) ("Double_Acting_Hammer"))

Listing D.1 Partial Listing of CMSA (continued) 


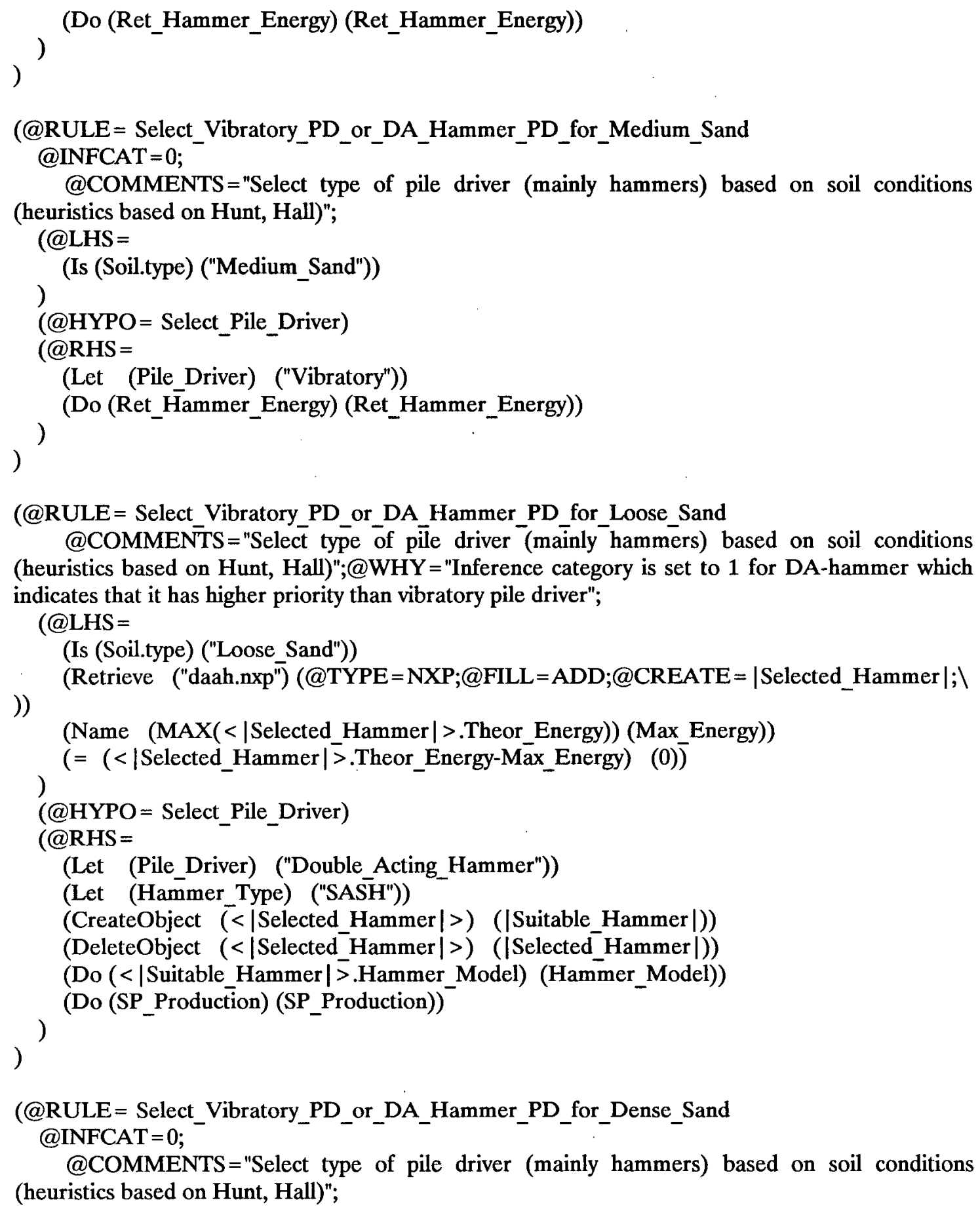

Listing D.1 Partial Listing of CMsA (continued) 


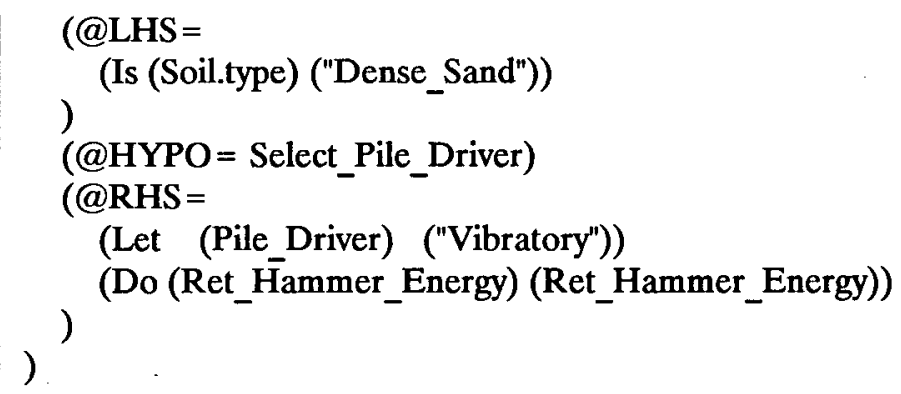

Listing D.1 Partial Listing of CMsA (continued) 


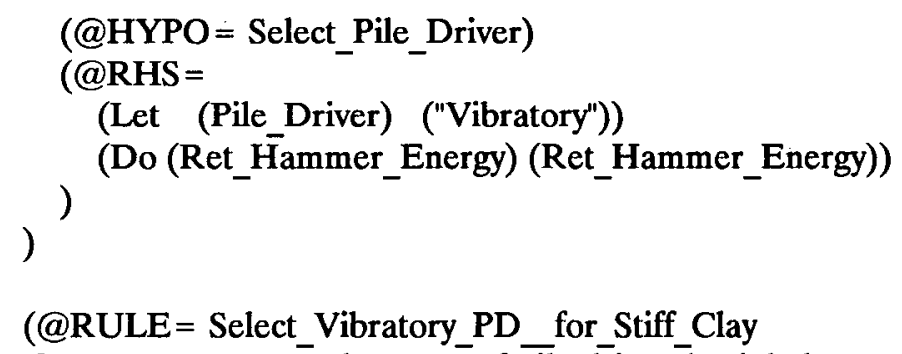

Listing D.1 Partial Listing of CMSA (continued) 


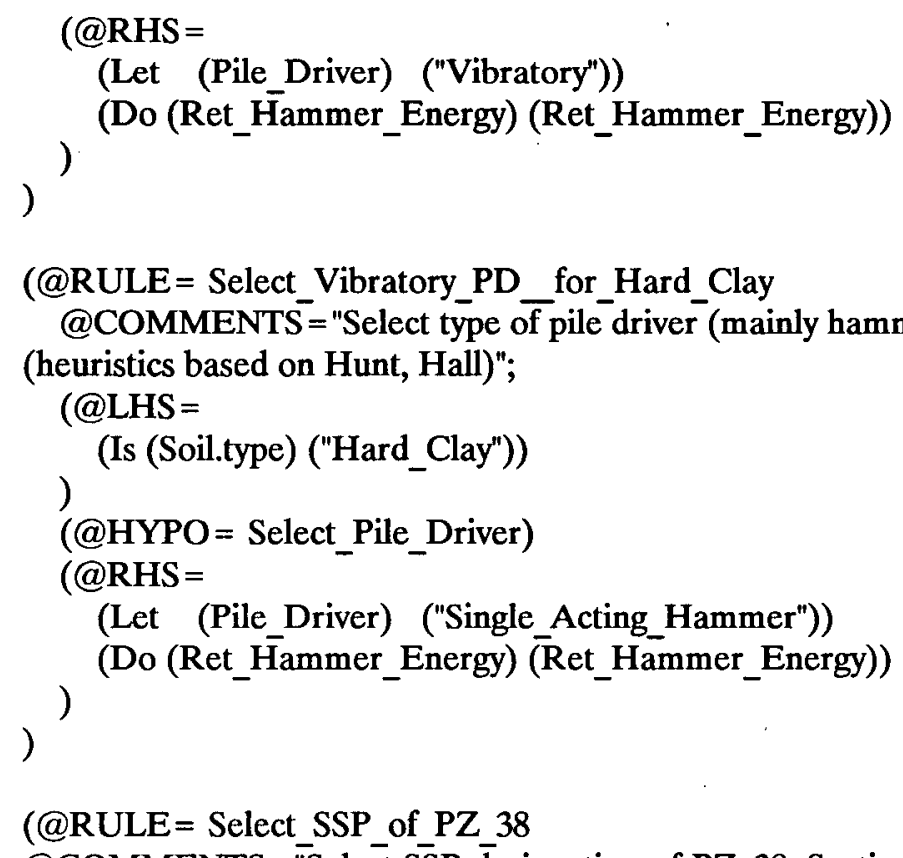
used to determine the type of sheet pile from which other properties can be deduced from the SSP data base (SSP.NXP) for further treatment.";

$(@$ LHS =

$(>=$ (Section_Modulus) (30.2))

(< (Section_Modulus) (38.3))

Listing D.1 Partial Listing of CMSA (continued) 


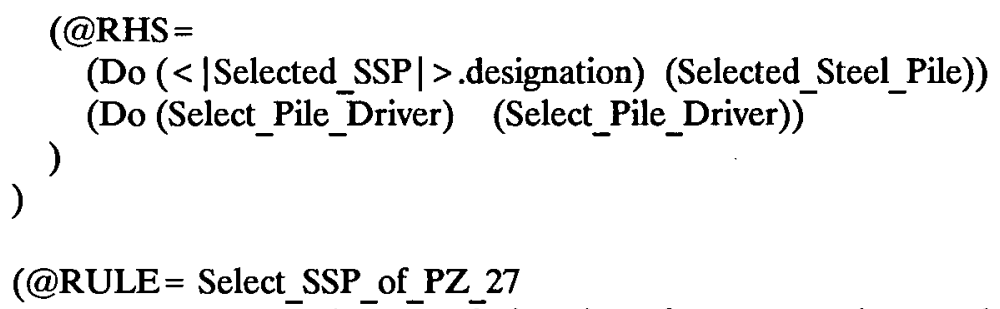
used to determine the type of sheet pile from which other properties can be deduced from the SSP data base (SSP.NXP) for further treatment.";

Iisting D.1 Partial Listing of CMSA (continued) 


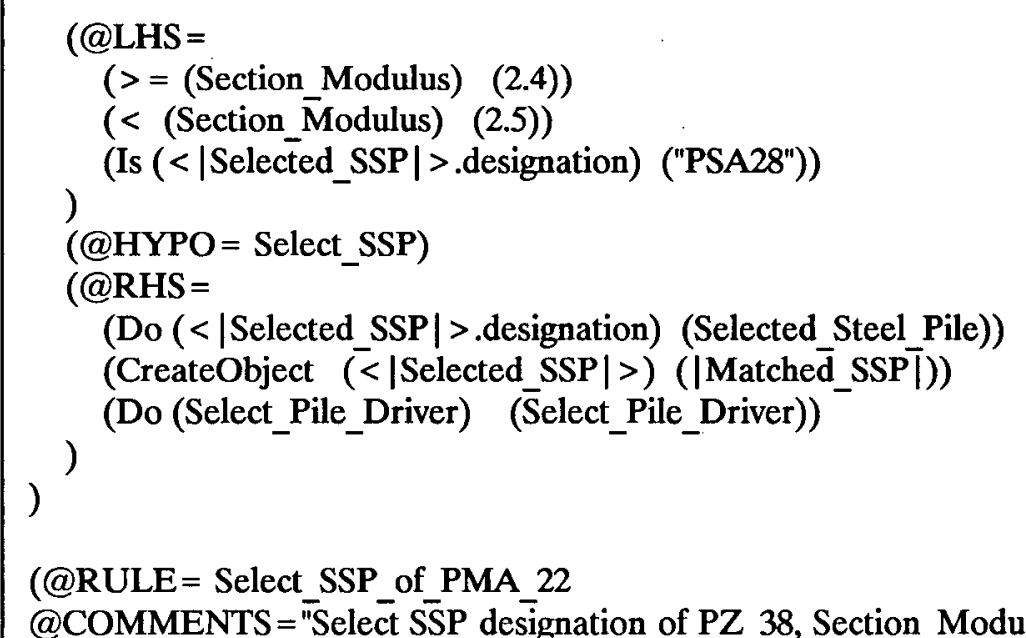
used to determine the type of sheet pile from which other properties can be deduced from the SSP data base (SSP.NXP) for further treatment.";

(@LHS=

( $>=$ (Section_Modulus) (2.5))

$(<$ (Section_Modulus) (5.4))

)

(Is (<|Selected_SSP $\mid>$.designation) ("PMA22"))

$(@ H Y P O=$ Select_SSP $)$

(@RHS =

(Do $(<\mid$ Selected_SSP $\mid>$.designation) (Selected_Steel_Pile))

(CreateObject $(<\mid$ Selected_SSP $\mid>)$ ( $\mid$ Matched_SSP $\mid))$

)

(Do (Select_Pile_Driver) (S̄elect_Pile_Driver))

)

$(@$ RULE $=$ Select_SSP_of_PMA22

$@$ @OMMENTS = "Select SSP designation of PZ_38, Section_Modulus is in in^3";@WHY="This is used to determine the type of sheet pile from which other properties can be deduced from the SSP data base (SSP.NXP) for further treatment.";

(@LHS =

( $>=$ (Section_Modulus) (2.5))

)

( $<$ (Section_-̄Modulus) (5.4))

$(@ H Y P O=$ Select_SSP $)$

(@RHS=

(Let (SSP) ("PMA22"))

(Do (Select_Pile_Driver) (Select_Pile_Driver))

Listing D.1 Partial Listing of CMSA (continued) 


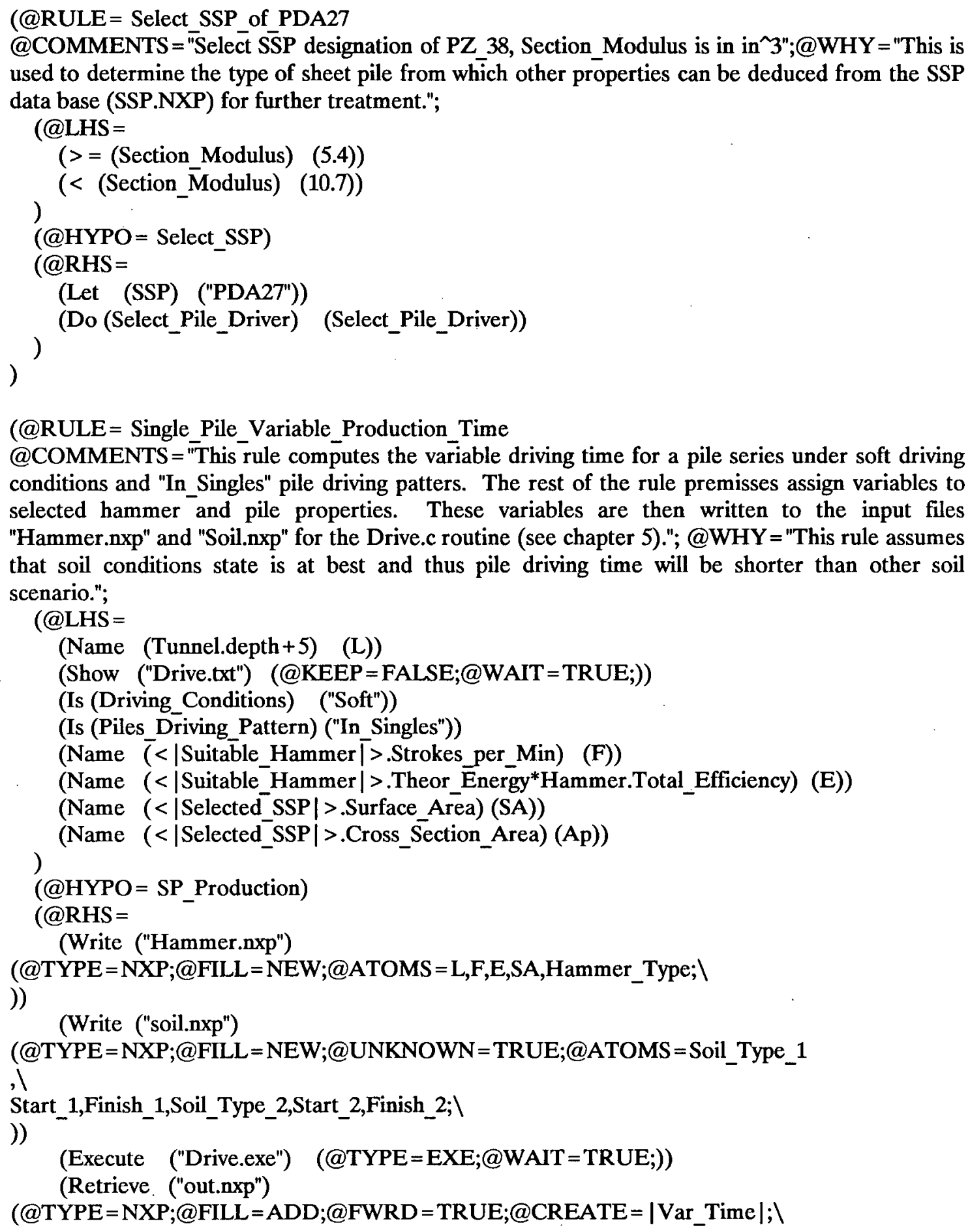

Listing D.1 Partial Listing of CMSA (continued) 


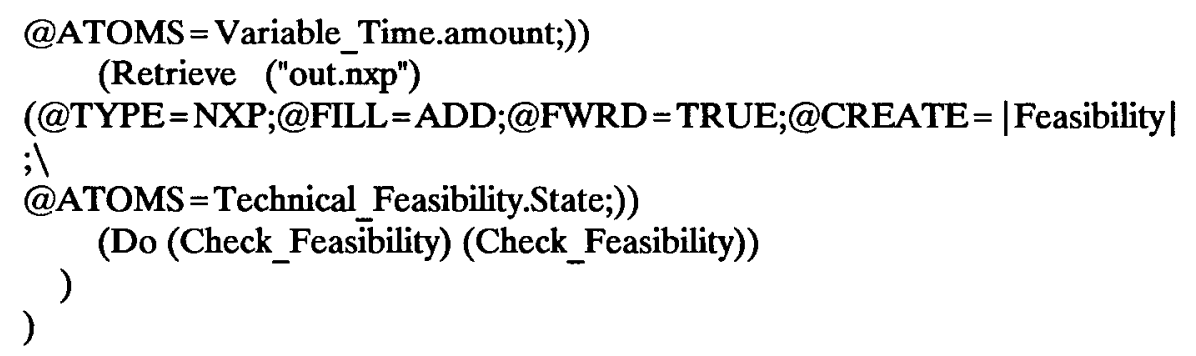

Listing D.1 Partial Listing of CMSA (continued) 


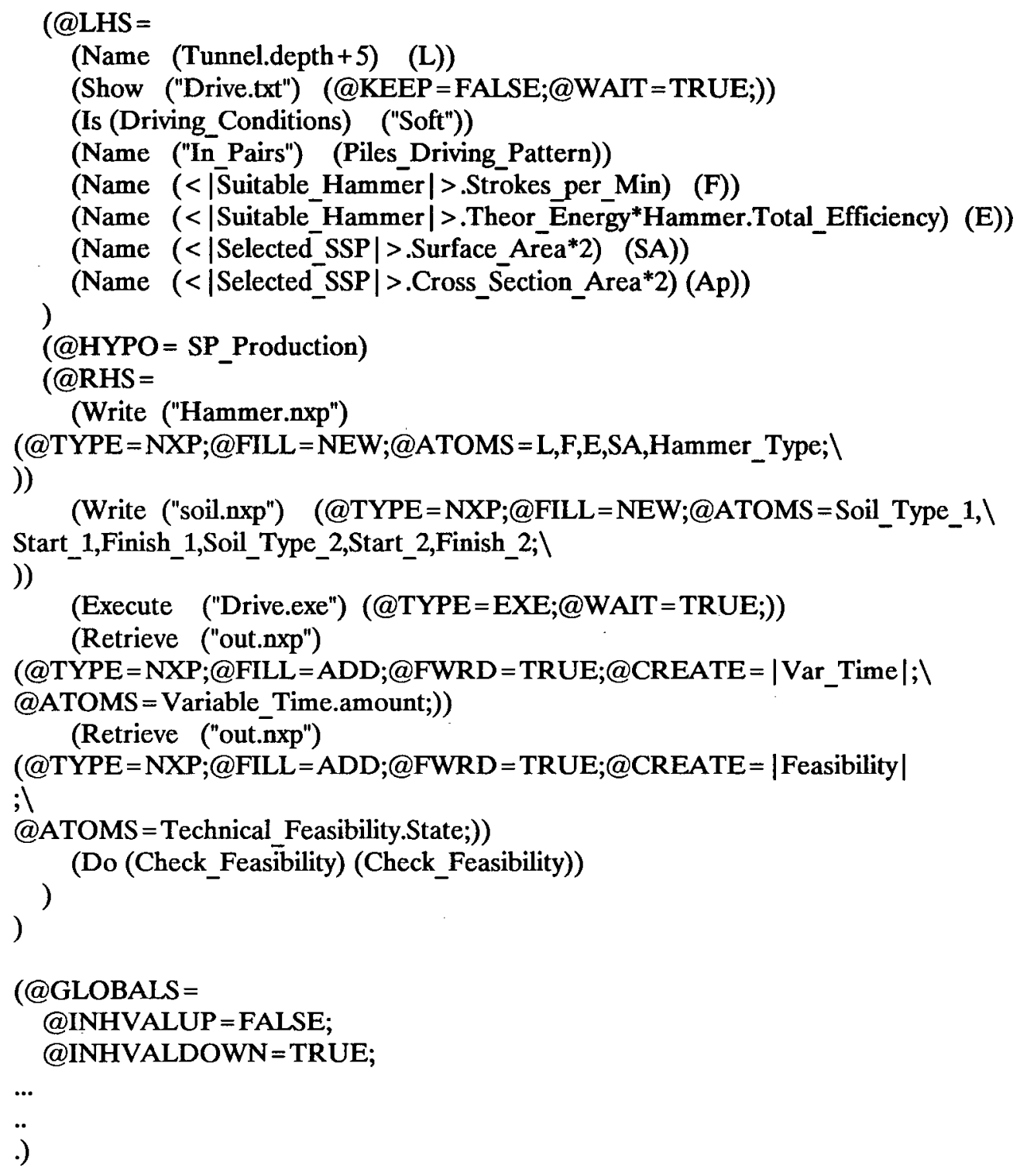

Listing D.1 Partial Listing of CMSA (continued) 


\section{D.3 Vibratory Hammer Selection Knowledge Base Development}

Vibratory pile driver selection knowledge was extracted from the literature. This component serves to select and size a vibratory hammer for a given soil and pile scenario. What is missing, is a model which can predict pile driving penetration rate as opposed to the dynamic formulas applied to the impact hammers. Furthermore, there is no indication what kind of vibratory pile driver suits a soil profile (as opposed to impact hammers types such as SAAH, DAAH, etc.). The interview with Quadra Construction Co. (see Appendix C) revealed that vibratory pile driver type selection is based on experience. In summary, there is no mechanism to check whether a pile driven by a vibratory pile driver could reach its refusal depth nor how long would it take to drive a pile to its refusal depth. Therefore, this part is limited to vibratory pile driver selection without a technical feasibility test.

The vibratory pile driver selection is based on empirical formula and charts found in Barber (1987) -- where a vibratory hammer can be specified at this level by its dynamic force and amplitude. From graph 1 of the previous reference, for a given soil profile SPT and different steel pile unit weights, one can find a vibratory dynamic force. Table D.1 presents vibratory dynamic force (in tons) computed in terms of SPT (substituted as $N$ ) against different pile unit weights as derived from Graph 1 (Barber 1979). For instance, the first relationship implies the following governing equation. For pile unit weight of 10 $1 \mathrm{~b} / \mathrm{ft}$,

Vibratory Dynamic Force $=0.21 * \mathrm{~N}$

where $\mathrm{N}$ is the standard penetration test (SPT).

If the pile unit weight is specified (as CMSA prototype specifies structural member of SSP/SPL), then the dynamic force for a suitable vibratory hammer can be determined, and vice versa. 


\begin{tabular}{|ccc} 
Line Number & Pile Unit Weight (lb/ft) & Vibratory Dynamic Forces \\
& & \\
1 & 10 & $0.21^{*} \mathrm{~N}$ \\
2 & 20 & $0.50 * \mathrm{~N}$ \\
3 & 30 & $0.75 * \mathrm{~N}$ \\
4 & 40 & $1.00 * \mathrm{~N}$ \\
5 & 50 & $1.20 * \mathrm{~N}$ \\
6 & 60 & $1.49^{*} \mathrm{~N}$ \\
7 & 70 & $1.72 * \mathrm{~N}$ \\
8 & 80 & $2.00^{*} \mathrm{~N}$ \\
9 & 90 & $2.33 * \mathrm{~N}$ \\
10 & 100 & $2.50 * \mathrm{~N}$ \\
11 & 110 & $2.70 * \mathrm{~N}$ \\
12 & 120 & $3.03 * \mathrm{~N}$ \\
13 & 130 & $3.23 * \mathrm{~N}$ \\
14 & 140 & $3.45 * \mathrm{~N}$ \\
15 & 150 & $3.85 * \mathrm{~N}$
\end{tabular}

Table D.1 Vibratory Pile Drivers sizing

Graph 2 of Barber (1979) presents a linear relationship between the pile length and vibratory amplitude. This is transformed into the following governing equation:

Amplitude $(i n)=$ Pile Length $(f t) * .016+0.12$ (D.2)

Using equation D.2, either the amplitude can be determined given a pile length, or vice versa. XMAS treats pile length (pile segment) as a variable volunteered by the user.

For the CMSA prototype, equation D.1 results in dynamic force designation of a vibratory size (where pile unit weight is predetermined by the hypotheses select_SSP and/or Select_SPL). Equation 2 specifies the vibrator $\bar{y}$ amplitude based on user pile segment size.

Crane Selection: cranes are considered as secondary resources for pile driving. Although crane selection depends on several factors, for CMSA prototype development, crane selection is determined by the weight of the hammer ram weight. Table D.2 presents rules of thumb for selecting cranes designated by their carrying capacity versus the theoretical energy of the impact hammer. For instance, a 35 ton crane may be used to carry a hammer with an upper bound 
of $8750 \mathrm{ft}-1 \mathrm{~b}, 40$ ton crane for hammers within the range $(8750-15000) \mathrm{ft}-1 \mathrm{~b}$, and so forth.

Cranes

(Ton)

25

40

60

100
Impact Hammer Energy

(lb-ft)

8750

15000

25000

$>25000$

Table D.2 Crane selection Format (From Means Heavy Construction Cost Data 1987)

"Crane.nxp" is the file that contains the crane data base that are used to represent cranes in CMSA prototype.

\section{4 Unit cost Quotations}

Cost quotation sources for the CMSA prototype include cost data manuals, previous projects, interviews, and local vendors. What follow are comments about cost estimates and their break down.

- Contractor experience from previous jobs was used to set upper and lower bounds for unit cost rates ( $x$ \$ ft run), or unit cost per surface area ( $x$ per square foot of steel sheet piling), and production rate in (day/ft).

- The interview with Quadra construction Co. (see Appendix C) provided experienced based estimates for the crews and equipment involved in the pile driving activity. For instance, from the project site visit, the following unit costs were obtained:

For sheet piling driving, the cost of material is approximately $3 / 4$ of the total cost of pile driving operation, whereas $1 / 4$ is labor cost and equipment (labor cost almost is almost equal to the equipment cost for a vibratory hammer).

Representative costs employed by CMSA are shown below in table D. 3 . 
1. Steel Sheet Piles

$$
\text { and } \mathrm{H} \text { - Piles }
$$

Grades (40 and $50 \mathrm{ksi}$ )

2. Lumber

Size

244
344
$4-4$
$4-6$
$6-6$

3. Impact Hammer

SAAH, DAAH

SAAH, DAAH

SAAH, DAAH

4. Crane

25 Ton

40 Ton

60 Ton

100 Ton

5. Crew (Pile Driving)

4 men / day

6. Vibrator Model

Model ICE 812

Model ICE 216
Unit Cost

$\$ 0.27 / \mathrm{ft}$

$\$ 0.50 / \mathrm{ft}$

$\$ 0.75 / \mathrm{ft}$

$\$ 1.35 / \mathrm{ft}$

$\$ 2.30 / \mathrm{ft}$

Theoretical

Energy (lb-ft)

8750

15,000

25,000
Unit Cost

$\$ 930 /$ Ton

Available Length

6 to $20 \mathrm{ft}$

6 to $20 \mathrm{ft}$

6 to $20 \mathrm{ft}$

6 to $20 \mathrm{ft}$

8 to $20 \mathrm{ft}$

Rent

$\$ 4000 /$ month $\$ 5000 /$ month $\$ 6000 /$ month

Rent

$\$ 5000 /$ month $\$ 6500 /$ month $\$ 9000 /$ month $\$ 11000 /$ month

Cost

$\$ 22000 /$ month

Rent

$\$ 11000 /$ month

$\$ 7000 /$ month

Table D.3 Representative Resources Unit costs in Vancouver, B.C, [Quadra construction Co Ltd]

In comparison with other shoring methods, for steel sheet piling, material cost savings are of major concern. Unit prices are as quoted from the local market (1990). These prices vary with the amount of material quantity, length and size of the piles/lumber segments, material quantity, and so forth. 


\section{D.5 Sample Data Base Files}

As indicated earlier, these include the design elements for the construction method (structural members (steel sheet piles, soldier piles, struts, wales, and laggings) + construction resources (hammers + cranes). The data bases contain a sample of what could be used. CMSA uses a subset of these data bases.

\section{1. steel sheet Piles}

The following properties and dimensions were taken from winterkorn and Fang (1975) and are found in database "SSP. nxp".

The SSP are divided into three groups according to their section modulus around the $\mathrm{X}$-axis (the assumption used is that the section modulus rather than interlock strength constitutes the basis for SSP selection including shape.

A partial listing of those used in CMSA is show in table D. 3 .

Group_1 : This is mainly z-section with Section Modulus (S):

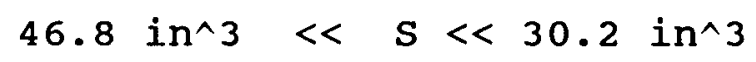

There are 3 SSP sections in this group.

Group_2: This is mainly invert U-section with section Modulus (S): 10.7 in^3 $^{\wedge} s \quad 2.4$ in^3 $^{\wedge}$

There are 2 SSP sections in this group.

Group_3: This is mainly PSA and PSA (straight) sections with Section Modulus (S):

$$
1.9 \operatorname{in}^{\wedge} 3 \quad<\quad s \quad<2.4 \text { in^3 }^{\wedge}
$$

There are 4 SSP sections in this group which depend on using them in applications involving interlock strength rather than section modulus. Properties are shown for information purposes.

Group_4: Miscellaneous sheet piling are not included.

Properties treated in the data base are as follows (listing D. 2) :

1. Designation;

2. Weight per foot in (Ib); 


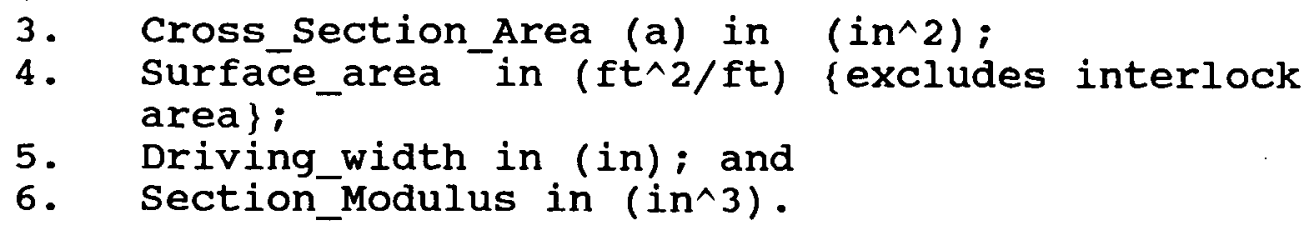

Group_1: Z-Section

\SSP 1.Designation $\backslash=$ "PZ38"

\SSP-1. Weight_per_foot $\backslash=" 57.00 "$

$\backslash$ SSP_1.Cross_section_area $\backslash=" 16.77 "$

$\langle$ SSP_1.Driving_width $\rceil=" 18^{\prime \prime}$

\SSP_1.Surface_area $\backslash=" 5.52 "$

$\backslash$ SSP_1.Section_modulus $\backslash=" 46.8 "$

\SSP 2.Designation $\backslash=$ "PZ32"

\SSP-2.Weight_per_foot $\backslash=" 56.00 "$

$\backslash$ SSP_2.Cross_section_area $\backslash=" 16.47 "$

$\backslash$ SSP_2.Driving_width $\backslash=" 21 "$

$\backslash$ SSP_2.Surface_area $\backslash=" 5.52 "$

$\backslash$ SSP_2.Section_modulus $\backslash=" 38.3 "$

\SSP 3.Designation \= "PZ727"

$\backslash$ SSP_3. Weight_per_foot $\backslash=" 40.5 "$

$\backslash$ SSP_3.Cross_section_area $\backslash=" 11.91 "$

$\backslash$ SSP_3.Driving_width $\backslash=" 18 "$

$\backslash$ SSP_3.Surface_area $\backslash=" 4.98 "$

\SSP_3.Section_modulus $\backslash=" 30.2 "$

...

..

-

\section{Listing D.2 sample of steel sheet Pile Data Base Used in CMSA "SSP. nXp"}

\section{Soldiers Piles}

Structural properties are given for use when standard HPPiles are utilized as rakes, wales or as other structural members (AISC 1978). The database "HP_Pile.nxp" contains selected soldier pile members.

The normal Material Specification is: ASTM A36, ASTM A572 grades 42 through 60 (HP $14 * 117$ is not available in grade 60). Those H-piles are available in welded form from Kaiser steel corporation. 
Note that other steel structural members such as WF-sections could be used. WF-sections have more flange width and thus are more resistant to lateral pressure. For the prototype application, attention was limited to $\mathrm{H}$-piles. Properties treated in the data base are as follows:

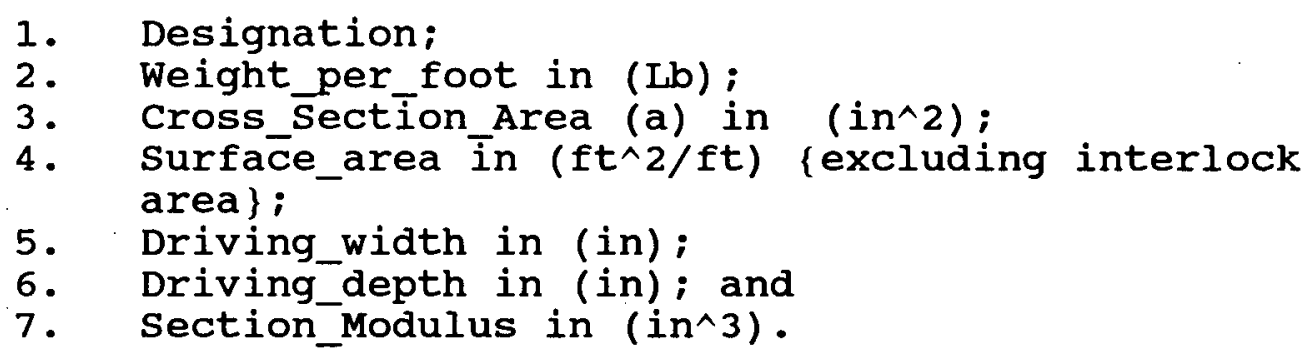

$\backslash$ HP-1.Designation $\backslash=" 14$ 117"

$\backslash$ HP-1.Weight_per_foot $\backslash=" 117 "$

$\backslash$ HP_1.Cross_section_area $\backslash=" 34.4 "$

$\backslash$ HP_1.Driving_width $\backslash^{-}=" 14.89 "$

$\backslash$ HP_1.Driving_Depth $\backslash=" 14.23 "$

$\backslash$ HP_1.Surface_area $\backslash=" 7.11 "^{-}$

$\mid \mathrm{HP}_{-}$1.Section_modulus $\backslash=" 173$ "

$\backslash$ HP-2.Designation $\backslash=$ "14_102"

$\backslash$ HP-2.Weight_per_foot $\backslash=" 102 "$

$\backslash$ HP_2.Cross_section_area $\backslash=" 30.0 "$

$\backslash$ HP_ $^{-}$.Driving_width $\backslash=" 14.78 "$

..

\section{Listing D.3 Soldier Piles sample Data Base "HP_Pile.nxp"}

\section{3. struts}

Properties and dimensions of American-Produced Standard (w) Shapes for Columns (Struts) and Beams (Wales) for Internal Bracing Retaining System (Table I-22 Manual of steel Construction, AISC) were used. The w14 series was adopted for the prototype because of the variety available in this size range. Further, $F y=36 \mathrm{ksi}$, and $\mathrm{Fa}=19 \mathrm{ksi}$ (Winterkorn and Fang 1975). The properties in the data base are as follows.

$$
\begin{aligned}
& \text { 1. Designation; } \\
& \text { 2. Weightper_foot in (Lb): } \\
& \text { 3. Cross_Section_Area }(\mathrm{a}) \text { in }\left(\mathrm{in}^{\wedge} 2\right) \text {; and } \\
& \text { 4. Radius_of_Gyration } \mathrm{r}(\mathrm{y}) \text { in (in). }
\end{aligned}
$$


\Strut_1.Designation \="W_14_132"

\Strut_1.Weight_per_foot $\backslash \overline{=}=\overline{13} 2.00 "$

|Strut_1.Cross_section_area $\backslash=" 38.8 "$

|Strut_1.Radius_of_Gyration $\backslash=" 3.76 "$

\Strut_2.Designation $\backslash=$ "W_14_120"

\Strut_2.Weight_per_foot $\backslash=" 1 \overline{2} 20.00 "$

Strut_2.Cross_section_area $\backslash=" 35.3 "$

|Strut_2.Radius_of_Gyration $\backslash=" 3.74 "$

$\backslash$ Strut_3.Designation $\backslash=" \mathrm{~W} 14$ 109"

\Strut_3.Weight_per_foot $\backslash=" 109.00 "$

$\backslash$ Strut_3.Cross_section_area $\backslash=" 32.0 "$

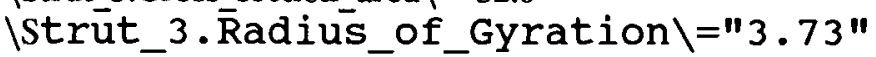

-..

$\cdots$

$\bullet$

\section{Listing D.4 struts Sample Data Base "Strut.nxp"}

\section{Lagging:}

structural properties are given for use when timber is utilized as lagging, rakers, wales or as other structural members (CSA standard 1976). Several nominal timber sizes are available such as $2 \_4,3 \_4,44_{4}, 4 \_2,4 \_3,4 \_6$, and 6_6 are stored in "lagging. nxp" database.

Properties pertinent to lagging design, within CMSA, are limited to:

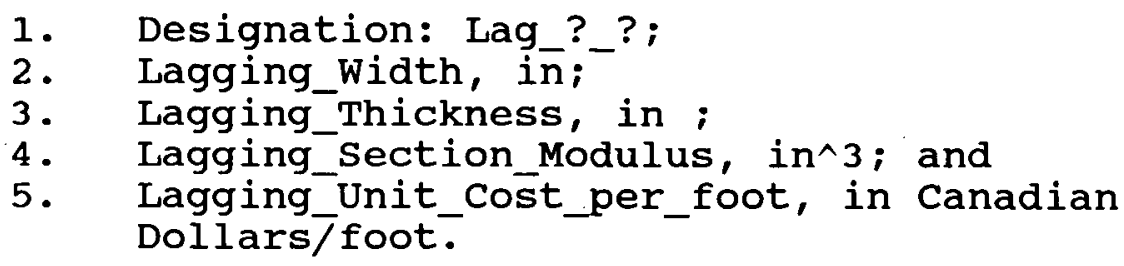


$\backslash$ Lagging_1.Designation $\backslash=$ "Lagging_2 4"

$\backslash$ Lagging_1.Width $\backslash=" 4 "$

$\backslash$ Lagging_1.Thickness $\backslash=" 2 "$

$\backslash$ Lagging_1.Section_modulus $\backslash=" 3.06 "$

\Lagging_1.Unit_Cost_per_Foot $\backslash=" 0.27 "$

\Lagging_2.Designation \="Lagging_3_4"

$\backslash$ Lagging_2.Width $\backslash=" 4 "$

$\backslash$ Lagging_2.Thickness $\backslash=" 3 "$

$\backslash$ Lagging_2.Section_modulus $\backslash=" 5.10 "$

$\backslash$ Lagging_2.Unit_Cost_per_Foot $\backslash=" 0.50 "$

...

..

\section{Listing D.5 Lagging sample Data Base "Lag.nxp"}

\section{Hammers sample}

Hammer databases are named according to the class of the hammer. For instance, "DAAH" stands for Double Acting Air Hammer; "SAAH" stands for single Acting Air Hammer, etc. Listing D.6 shows a sample of impact hammers which is implemented in CMSA.

Table 17-16 of Peurifoy (1970) data on pile driving hammers has been adopted for the impact hammer data base. Data fields used are: :

1. Ram Weight in (1b);

2. Strōke_per_minute in (no units);

3. Length of stroke in (in); and

4. Theoretical_Energy (ft-lib) per blow.

This database contains single acting air hammers of type vulcan. 
$\backslash$ Hammer_01.Hammer_Model $\backslash=" 2 "$

$\backslash$ Hammer_01.Ram_Weight $\backslash=" 3000 "$

$\backslash$ Hammer_01.Strokes_per_Min $\backslash=" 70 "$

$\backslash$ Hammer_01.Length_of_Stroke $\backslash=" 29 "$

$\backslash$ Hammer_01.Thero_Energy $\backslash=" 7260 "$

$\backslash$ Hammer_02.Hammer_Model $\backslash=$ "1"

$\backslash$ Hammer_02.Ram_Weight $\backslash=" 5000 "$

$\backslash$ Hammer_02.Strokes_per_Min $\backslash=" 60 "$

$\backslash$ Hammer_02.Length_of_Stroke $\backslash=" 36 "$

$\backslash$ Hammer_02.Thero_Energy $\backslash=" 15000 "$

$\backslash$ Hammer_03.Hammer_Model $\backslash=$ "0"

$\backslash$ Hammer_03.Ram Weight $\backslash=" 7500 "$

$\backslash$ Hammer_03.Strokes_per_Min $\backslash=" 50 "$

$\backslash$ Hammer_03.Length_of Stroke $\backslash=" 39 "$

$\backslash$ Hammer_03.Thero_Energy $\backslash=" 24375 "$

\section{Listing D.6 Impact Hammer Sample Data Base "Hammer.nxp"}

\section{Vibratory Hammers sample}

"Vibro.nxp" is the database for variety of vibratory pile drivers. The following different models of vibratory hammers are adopted from Peurifoy (1970) for Foster Vibro driver/extractor (1isting D.7) . Properties of interest are:

1. Maximum Energy delivered, ft-lb per sec;

2. Vibration frequency, rpm, min;

3. Vibration frequency, rpm, max;

4. Total horsepower;

5. Voltage;

6. Maximum Amplitude;

7. Cycles per sec, and; and

8. Approximate weight, $1 \mathrm{~b}$. 
\Vibratory_1.Model $\backslash=" 2-17 "$

|Vibratory_1.Max_Energy \="18440"

|Vibratory_1.Min_Frequency $\mid=" 1090 "$

|Vibratory_1.Max_Frequency $\mid=" 1290 "$

|Vibratory_1.Tot _Horsepower $\backslash=" 34 "$

$\mid$ Vibratory_1. Voltage $\backslash=" 440 "$

\Vibratory_1.Max_Amplitude $\backslash=" 60 "$

|Vibratory_1.Cycles $\backslash=" 60 "$

|Vibratory_1.Approx_Weight $\backslash=" 6200 "$

|Vibratory_2.Model $\backslash=" 2-35 "$

|Vibratory_2.Max_Energy $\backslash=" 37970 "$

|Vibratory_2.Min_Frequency $\backslash=" 890 "$

|Vibratory_2.Max_Frequency $=" 1120 "$

|Vibratory_2.Tot_Horsepower $\backslash=" 70 "$

$\mid$ Vibratory_2.Voltage $\backslash=" 440 "$

|Vibratory_2.Max_Amplitude $\backslash=" 120 "$

|Vibratory_2.Cycles $\backslash=" 60 "$

\Vibratory_2.Approx_Weight $\backslash=" 9100 "$

Listing D.7 Vibratory Hammers Sample Data Base [Vibro.nxp] 